\title{
Design und Synthese neuer flüssigkristalliner Substanzen mit zentraler Bicyclo[3.1.0]hexaneinheit
}

\author{
Dissertation \\ Zur Erlangung des Doktorgrades \\ der Mathematisch-Naturwissenschaftlichen Fakultäten \\ der Georg-August-Universität zu Göttingen
}

vorgelegt von

Rainer Langer

aus Püttlingen

Göttingen 2003 
Die vorliegende Arbeit wurde am Institut für Organische Chemie der Georg-AugustUniversität zu Göttingen unter der Leitung von Herrn Prof. Dr. Armin de Meijere in der Zeit vom Mai 2000 bis zum Mai 2003 angefertigt.

Ich danke Herrn Prof. Dr. Armin de Meijere für die Überlassung der interessanten Themenstellung, Herrn Prof. Dr. Dietrich Demus für die kreative Zusammenarbeit bei dem Design der hier beschriebenen neuen Substanzklassen, Herrn Dr. Sergei I. Kozhushkov und Herrn Dr. Andrei Leonov für die unermüdliche Diskussion synthetischer Probleme und dem L.C. Research Center der Firma Chisso Petrochemical Corporation unter der Leitung von Dr. Kazutoshi Miyazawa für die Überlassung von Bausteinen zur Flüssigkristallsynthese, die finanzielle Unterstützung beim Kauf von Katalysatoren und Reagenzien und die Patentierung der neuen Bicyclo[3.1.0]hexane. 
Ref: Prof. Dr. A. de Meijere

Korr: Prof. Dr. H. Laatsch 
Meinen Eltern. 


\section{Inhaltsverzeichnis}

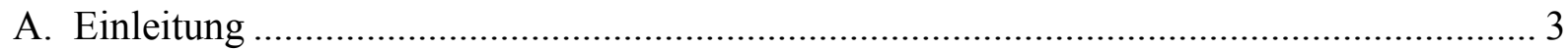

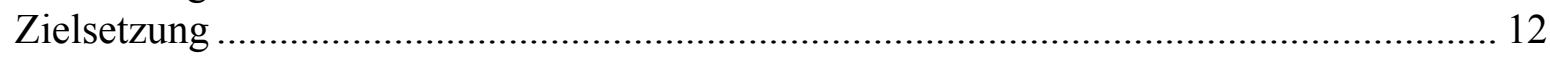

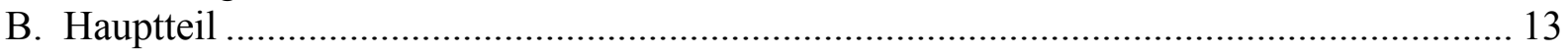

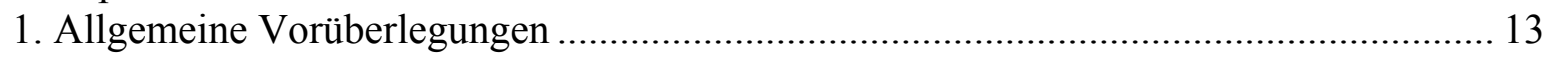

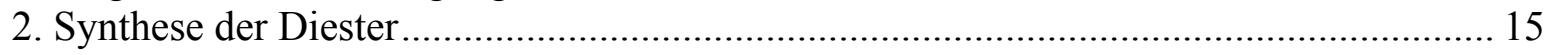

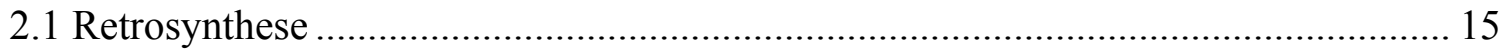

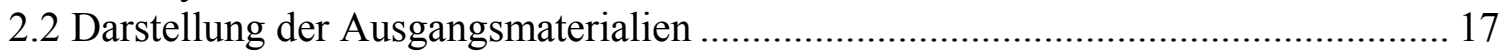

2.3 Synthese der Bicyclo[3.1.0]hexan-3,6-dicarbonsäurediester................................. 20

2.4 Diskussion der Eigenschaften von exo,exo-Bicyclo[3.1.0]hexan-3,6-dicarbonsäure-

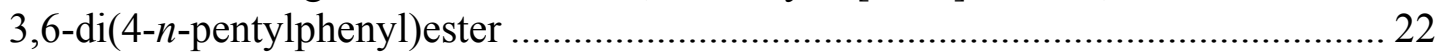

3. Synthesewege für 3,6-disubstituierte Bicyclo[3.1.0]hexanderivate als Mittelstück ........ 24

4. Retrosynthetische Überlegungen zu Bicyclo[3.1.0]hexanderivaten ............................. 27

4.1 Die Synthese von 2-Oxo-6-phenyl-3-oxabicyclo[3.1.0]hexan ................................ 28

4.2 Synthese von 6-(4-Bromphenyl)-2-oxo-3-oxabicyclo[3.1.0]hexan ......................... 32

4.3 Versuche zur Ringschlussreaktion von 2-Hydroxymethyl-3-

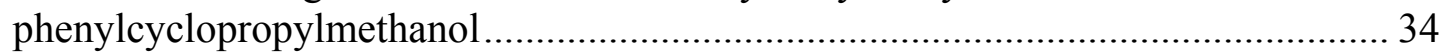

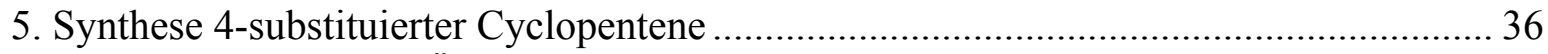

5.1 Retrosynthetische Überlegungen zu 4-substituierten Cyclopentenderivaten.............. 36

5.2 Palladium-katalysierte Aryl-Cyclopentenyl-Kreuzkupplung................................. 37

5.2 Cuprat-katalysierte Alkyl-Cyclopentenyl-Kreuzkupplung .................................... 40

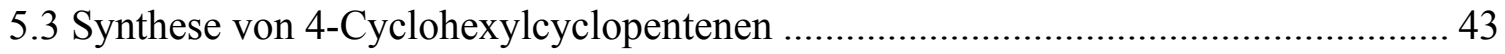

5.3.1 Vorüberlegungen und synthetische Ansätze .................................................. 43

5.3.2 Versuche zur Synthese von Cyclohexylcyclopentenylderivaten aus $\delta$ -

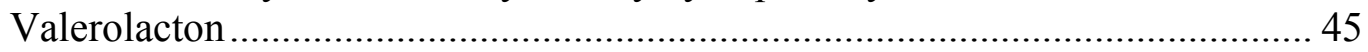

5.3.3 Cyanacetamidkondensation mit nachfolgender Michael-Addition..................... 47

5.3.4 Synthese eines 3-Alkyl-1,5-diylbismagnesiumbromids mit nachfolgender

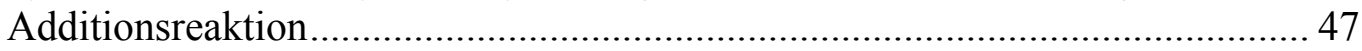

6. Synthese und Addition von Carbenoiden an 4-substituierte Cyclopentenderivate .......... 50

6.1 Die Erzeugung von Chlorcarbenen aus Cycloalkylmethanol- oder

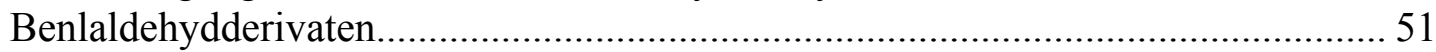

6.2 Synthese von Diazomethanderivaten aus $N$-Nitrosoharnstoffderivaten ................... 52

6.3 Synthese von Phenyldiazomethanderivaten aus Tosylhydrazonen............................ 54

7. Konvergente Synthese neuer flüssigkristalliner Substanzen mit zentraler

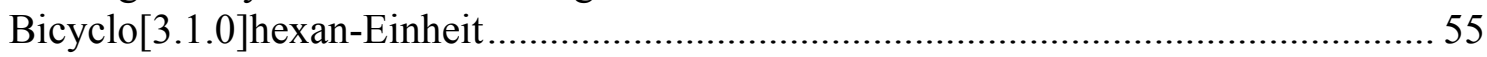

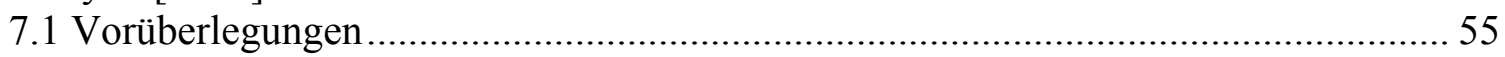

7.2 Die Erzeugung und Versuche zur Addition von Chlorcarbenen............................... 56

7.3 Katalysierte Additionen von Phenyldiazomethanderivaten ....................................5

7.3.1 Allgemeine Vorüberlegungen über geeignete Katalysatoren …......................... 58

7.3.2 Katalysierte Additionen von Aryldiazomethanderivaten an 4-Alkylcyclopentene59

7.4 Photochemische Spaltung von Aryldiazomethanderivaten.........................................6 62

7.5 Addition von tert-Butyldiazoacetat mit nachfolgender Homologisierung................. 63

C. Diskussion der Ergebnisse: Synthetische Resultate und physikalische Eigenschaften der

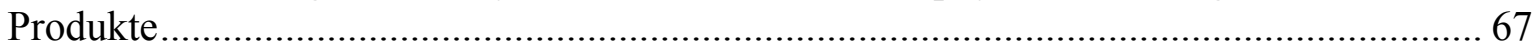

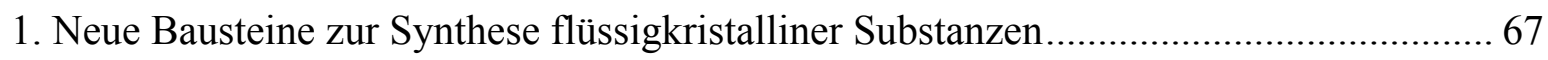

2. Eigenschaften der neuen Substanzen mit zentraler Bicyclo[3.1.0]hexan-Einheit .......... 69

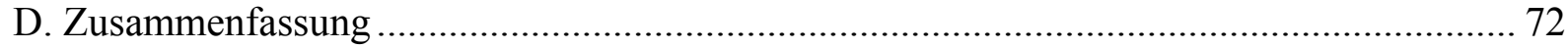




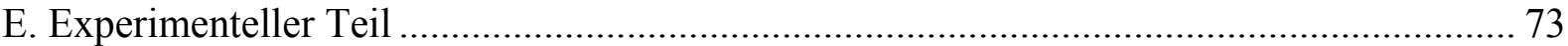

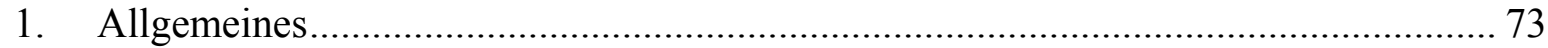

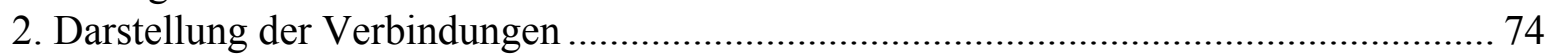

2.1 Allgemeine Arbeitsvorschriften ........................................................................ 74

2.2 Beschreibung der Synthesen .............................................................................. 77

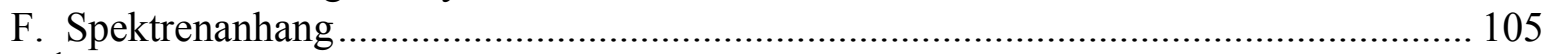

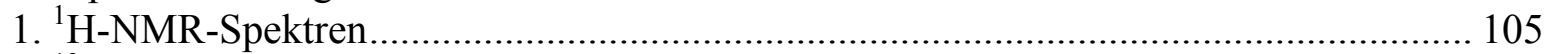

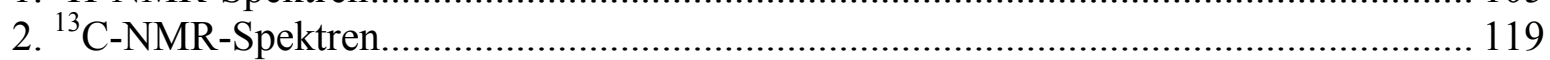

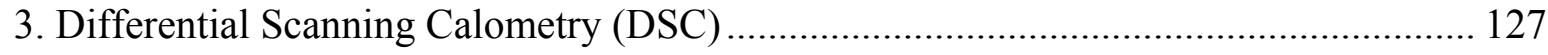

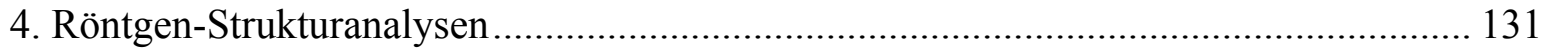

4.1. exo,exo-Bicyclo[3.1.0]hexan-3,6-dicarbonsäure-3,6-di(4-n-pentylphenyl)ester (35a)131

4.2. 3-\{[4-(trans-4- $n$-Pentyl)cyclohexyl]-phenyl\}bicyclo[3.1.0]hexan-6-carbonsäure-tert-

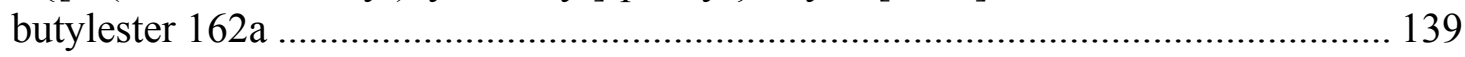

4.3. exo,exo-3-(3,4,5-Trifluorophenyl)bicyclo[3.1.0]hexan-6-carbonsäure-tert-butylester $162 \mathrm{~b}$

4.4. endo,exo-6-(4-Cyanophenyl)-3-n-pentylbicyclo[3.1.0] hexan 155b .......................... 153

4.5 exo,exo-6-(4-Cyanophenyl)-3- $n$-pentylbicyclo[3.1.0] hexan 155a ............................... 161

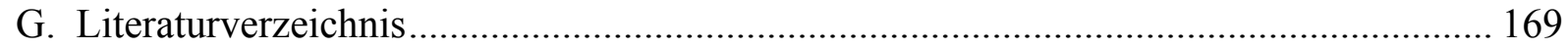




\section{A. Einleitung}

In unserem informationsbasierten Zeitalter wird die Verbreitung und Anzeige von Informationen zunehmend wichtiger. Man benötigt Geräte zur Darstellung dieser Informationen. An diese Geräte werden einige Anforderungen gestellt: Sie sollen eine möglichst hohe Informationsdichte aufweisen, langlebig sein, einen niedrigen Energieverbrauch haben, und Informationen schnell übermitteln.

Wegen all dieser Anforderungen verdrängen die neuen flüssigkristallbasierte Anzeigetechniken die konventionellen Kathodenstrahlröhren zunehmend. Die wichtigsten Nachteile wie hoher Energieverbrauch und eine Belastung des Betrachters durch Röntgenstrahlung konnten durch diese neuen Techniken behoben werden.

Bekanntermaßen geht die Entdeckung der Flüssigkristalle zurück auf den Prager Botaniker Freidrich Reinitzer ${ }^{[1]}$, der das Phänomen des doppelten Schmelzpunkts von Cholesterinbenzoat beobachtete. Als er das dem deutschen Physiker Lehmann mitteilte, stellte dieser fest, dass bei der Dunkelfeldmikroskopie der Mesophase Brechungsphänomene zu beobachten $\operatorname{sind}^{[2]}$. Während der nächsten 80 Jahre war die Entdeckung und Erforschung von ausschließlich akademischem Interesse. Im Jahr 1968 berichtete Heilmeier et al. über den Dynamic scattering effect (DS) aufgrund der elektrooptischen Eigenschaften der Flüssigkristalle, was eine mögliche technische Anwendung des Phänomens in Aussicht stellte $^{[3]}$. Seit dieser Zeit wurden viele flüssigkristalline Substanzen synthetisiert und in Liquid Crystal Displays (LCDs), der derzeitigen, und vermutlich zukünftigen Hauptanwendung, eingesetzt. Seit einigen Jahren sind Flüssigkristallbildschirme wegen ihrer geringen Bautiefe und ihrer Ökonomie für viele Anwendungen wie etwa für Fernseher, Notebooks und Videobeamer interessant geworden.

Die Mesophase von Flüssigkristallen ist definiert als ein Zustand, in dem der Ordnungsgrad der Moleküle höher ist, als in einer Flüssigkeit, aber niedriger als in einem Kristall. Wenn eine Substanz diese Eigenschaft besitzt, weist sie flüssigkristalline Eigenschaften auf. Dabei existiert keine Fixierung der Moleküle an einem definierten Ort, wie in Kristallen, aber eine Ordnung hinsichtlich der Ausrichtung der Moleküle (Director). Eine bestimmte Mesophase ist über einen stoffspezifischen Temperaturbereich stabil, abhängig vom Ordnungsgrad der Moleküle. Die verschiedenen Phasen flüssigkristalliner Substanzen sind in viele Klassen unterteilt. Die wichtigsten darunter sind die nematische Phase $(\mathrm{N})$ und die smektische Phase 
(smC). Der Nachweis des jeweiligen Zustandes gelingt durch Röntgenbeugung. Es hat sich gezeigt, dass langgestreckte, stäbchenförmige Moleküle wünschenswerte Eigenschaften besitzen und die Mehrzahl der bekannten mesogenen Substanzen weisen eine solche Molekülgeometrie auf. Diese Molekülgeometrie ist aber keinesfalls Vorraussetzung für die Ausbildung von Mesophasen, wie jüngst duch die Synhtese von „Bowl-shaped liquid crystals $^{\text {“[4] }}$ oder „Banana liquid crystals ${ }^{\text {“[5] }}$ gezeigt werden konnte. Stäbchenförmige Moleküle (rod-shaped) bestehen im Allgemeinen aus einem festen Mittelstück, und bewegicheren Substituenten am Ende, die mit einem Linker an das Mittelstück gebunden sind. Gewöhnlich besteht das Mittelstück aus einem meist sechsgliedrigen Ring wie Benzol, Cyclohexan, Pyrimidin, Dioxan, oder aus Kombinationen daraus. Die Endgruppen können gesättigte, oder ungesättigte, normale oder verzweigte Alkylketten sein und tragen oft polare Gruppen wie Halogene oder Cyanogruppen.

Die zweifelsohne wichtigste Funktion von Flüssigkristallen ist ihre Verwendung in Bildschirmen. Seit 1968 wurden nicht nur flüssigkristalline Substanzen, sondern auch der technische Aufbau für die Steuerung der Displays erforscht. Im Jahr 1963 berichtete Williams über den „stripped domain effect“ flüssigkristalliner Substanzen im elektrischen Feld, und Wysocki ${ }^{[6]}$ et al. fanden 1968 den Phasenübergang von cholesterischen Flüssigkeiten. Heilmeier et al. schlugen 1968 vor, Displays zu konstruieren, die den dynamic scattering effect und den guest-host-effect ausnutzen. Im Jahr 1971 schlugen Schadt et al. ${ }^{[7]}$ vor, den twisted nematic effect (TN) in den Displays zu nutzen, was zu einer sehr wichtigen Anwendung führte und zur einfachsten und heute meist benutzten Bildschirm-konstruktion führte. Die aktuellen Forschungsbemühungen haben sich zum Ziel gesetzt, die Eigenschaften der bekannten Displays hinsichtich des Engergieverbrauchs, der chemischen Stabilität, und der Anzeigegeschwindigkeit weiter zu verbessern. Man wird zwar mit Substanzen, die sich in einem elektrischen Feld ausrichten, wegen ihrer Trägheit und Viskosität niemals eine so hohe Geschwindigkeit erzielen, wie das mit dem Elektronenbeschuss in einer Brownschen Röhre der Fall ist. Sollte es aber erreicht werden können, dass das menschliche Auge die Umschaltzeit nicht mehr wahrnehmen kann, ist das Entwicklungsziel erreicht. 


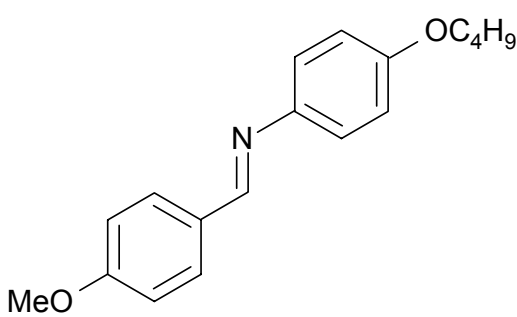

1

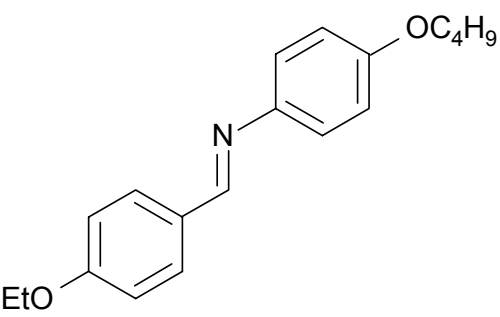

2

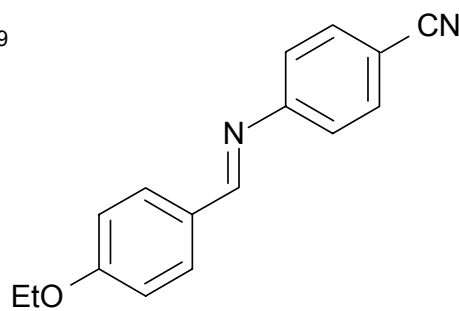

Abb. 1. Beispiele für erste praktisch eingesetzte Flüssigkristalle.

MBBA ( $p$-Methoxybenzyliden- $p$ '-butyloxyanilin) (1), und EBBA ( $p$-Ethoxybenzyliden- $p$ 'butyloxyanilin) (2) wurden von Kelker et al. ${ }^{[8]}$ synthetisiert und als erste Substanzen für LCDs verwendet. Die Substanzen zeigen eine nematische Phase bei Zimmertemperatur, MBBA zeigt beispielsweise die nematische Phase in einem Temperaturbereich von $20-41$ ${ }^{\circ} \mathrm{C}$. Die Substanzen haben eine negative dielektrische Anisotropie und sind geeignet für DSDisplays. Zur Konstruktion von TN-Displays sind Substanzen mit einer großen positiven dielektrischen Anisotropie nötig ${ }^{[9]}$. PEBAB ( $p$-Ethoxybenzyliden- $p$ '-aminobenzonitril) 3 und deren Homologe sind im Jahr 1970 vorgeschlagen worden. Diese Substanzen haben eine große positive dielektrische Anisotropie und können erfolgreich in TN-Displays eingesetzt werden. Diese ersten Substanzen waren für das Betreiben der Displays unter technischem Aspekt befriedigend, unter chemischen Aspekt haben sie den Nachteil, dass sie wegen der Iminofunktion als Kupplungsgruppe nicht hydrolysebeständig sind.
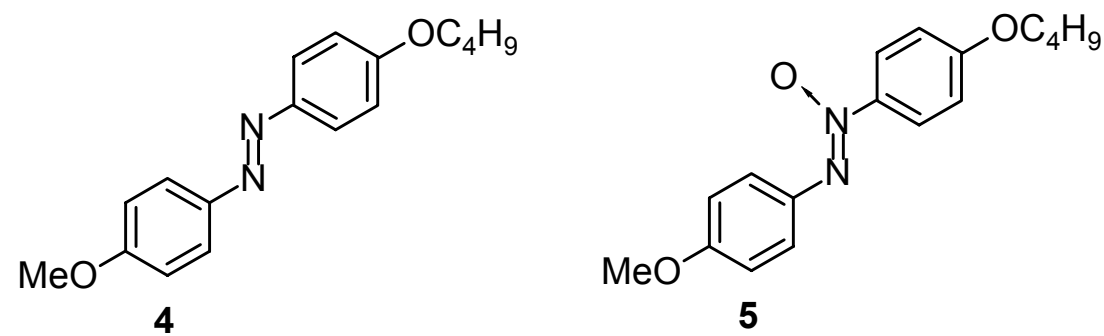

Abb. 2. Beispiele für hydrolysebeständige praktisch angewandte Flüssigkristalle.

Die alternativ vorgeschlagenen trans-konfigurierten Azo- und Azoxysubstanzen $\mathbf{4}$ und $\mathbf{5}$ sind chemisch stabiler und weisen eine nematische Phase $(\mathrm{N})$ in ähnlichen Temperaturbereichen auf $^{[10]}$. Die Verbindung 5 zeigt beispielsweise eine nematische Phase im Temperaturbereich von $41-69^{\circ} \mathrm{C}$. Ein Nachteil dieser Substanzklasse ist ihr Absorptionsmaximum bei $400-$ $450 \mathrm{~nm}$, weshalb die Substanzen photolabil sind. Durch Bestrahlung mit der entsprechenden Wellenlänge können die trans-konfigurierten Azo- und Azoxylinker teilweise in die 
entsprechenden cis-konfigurierten Isomere umgewandelt werden, die keine Mesophase aufweisen. Die entsprechenden Displays müssen demnach einen zusätzlichen Film enthalten, der die Substanzen vor Bestrahlung der entsprechenden Wellenlänge schützt.

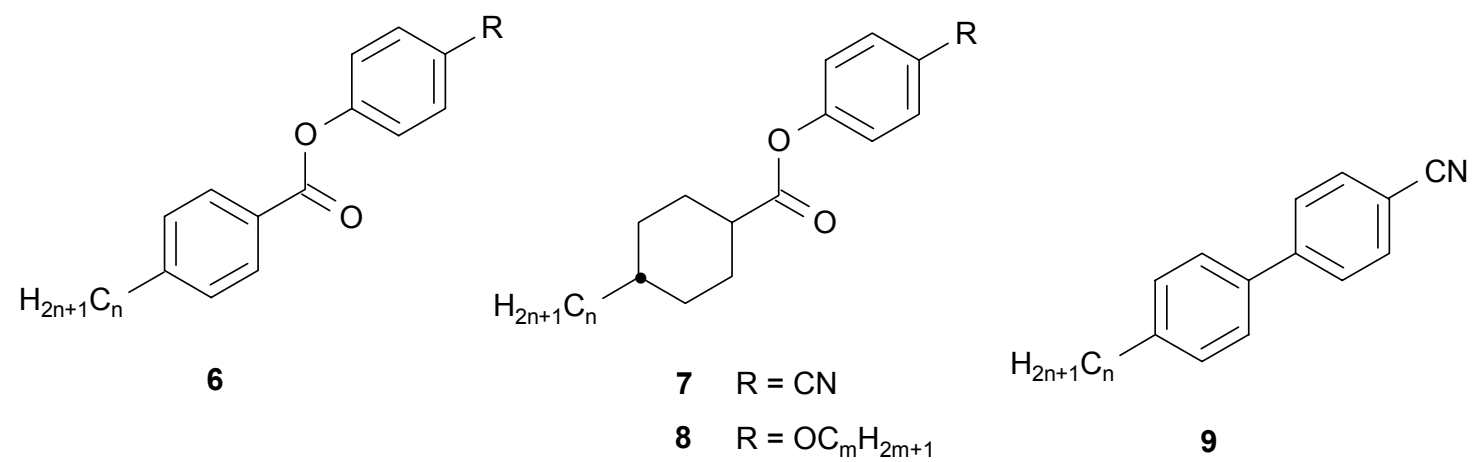

Abb. 3. Erste chemisch und photochemisch stabile Flüssigkristalle.

Als erste chemisch stabile Flüssigkristalle wurden in den frühen siebziger Jahren 4-Cyanophenyl-4'-alkylbenzoate vorgeschlagen. Verbindung 6 zeigt eine extrem große positive dielektrische Anisotropie ( $>20)$ und eine binäre Mischung aus Butyl- und Heptylderivaten besitzt eine nematische Phase im Temperaturbereich zwischen $25-50{ }^{\circ} \mathrm{C}$. Die entsprechenden Displays zeichnen sich durch einen hohen Kontrast und eine niedrige Betriebsspannung aus.

Im Jahr 1974 wurden von der Gruppe von Demus ${ }^{[11]}$ flüssigkristalline Substanzen mit 4-Cyanophenyl-4'-alkylcyclohexan-1-carboxylaten 7 und 4-Alkoxyphenyl-4'-alkylcyclohexan-1-carboxylaten 8 beschrieben. Diese Substanzen zeigen eine nematische Phase bei Raumtemperatur und eine wegen der im Vergleich $\mathrm{zu}$ den zuvor beschriebenen $\pi$-elektronenreichen Aromaten $\pi$-elektronenarmen Cyclohexan-Einheit relativ kleine optische Anisotropie. Besonders Verbindung 8 besitzt eine extrem geringe Viskosität und ist sehr nützlich als Basis für flüssigkristalline Mischungen, die auch zur Zeit noch in TN-LCDs benutzt werden.

Fast gleichzeitig beschrieb die Gruppe von Gray ${ }^{[12]}$ das 4'-Alkyl-4-cyano-1,1'-biphenyl 9, das ebenfalls eine nematische Phase bei Raumtemperatur, niedrige Viskosität, hohe optische und dielektrische Anisotropie und eine hohe chemische Stabilität aufweist. Auch diese Substanzen sind von industrieller Bedeutung, und in vielen der LCDs sind diese Substanzen als Basis enthalten. 


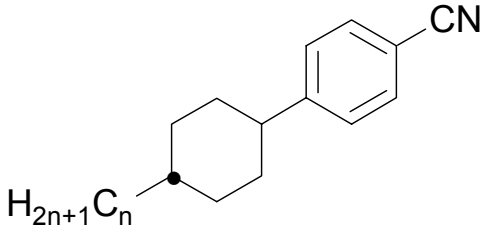

10

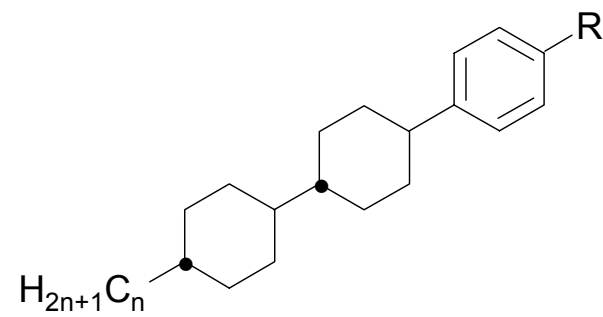

$11 \mathrm{R}=\mathrm{CN}$

$12 \mathrm{R}=\mathrm{OC}_{\mathrm{m}} \mathrm{H}_{2 \mathrm{~m}+1}$

Abb. 4. Weiterentwickelte flüssigkristalline Substanzen.

Mit der zunehmenden Entwicklung der Steuertechnik für die LCDs stiegen die Ansprüche an flüssigkristalline Substanzen. Zu den Anforderungen zählen größere Temperaturbereiche für die nematische Phase einschließlich tiefen Temperaturen bis $-30^{\circ} \mathrm{C}$ und geringere Viskositäten für schnellere Schaltzeiten ${ }^{[13]}$. Der Meilenstein in der Geschichte der Flüssigkristalle wurde durch die PCHs 4-(4-Alkylcyclohexyl)benzonitrile 10 gesetzt, die 1977 von Eidenschink et al. ${ }^{[14]}$ synthetisiert wurden.

Diese Substanzen zeigen eine nematische Phase bei Raumtemperatur und eine extrem niedrige Viskosität, die nur die Hälfte des Wertes von 4'-Alkyl-4-cyano-1,1'-biphenylen 9 beträgt. Die Substanzen vom Typ 10 sind eine der wichtigsten Klassen in der industriellen Fertigung für LCDs geworden. Die 1982 - 1983 von Chisso Corporation ${ }^{[15]}$ entwickelten Substanzen mit einer kettenförmigen Verknüpfung von Ringen, wie in 4-[4-(4Alkylcyclohexyl)cyclohexyl]benzonitrilen 11 und 4-[4-(4-Alkylcyclohexyl)cyclohexyl]alkoxybenzol 12 besitzen nematische Phasen in höheren Temperaturbereichen, niedrige Viskositäten, und eine sehr gute Kompatibilität mit anderen flüssigkristallinen Substanzen. Durch eine Mischung aus 10, 11 und 12 kann eine eutektische Mischung mit einer sehr breiten nematischen Phase $\left(-30-70^{\circ} \mathrm{C}\right)$ erhalten werden, deren weitere Vorteile schnelle Schaltzeiten und niedrige Schaltspannungen sind.

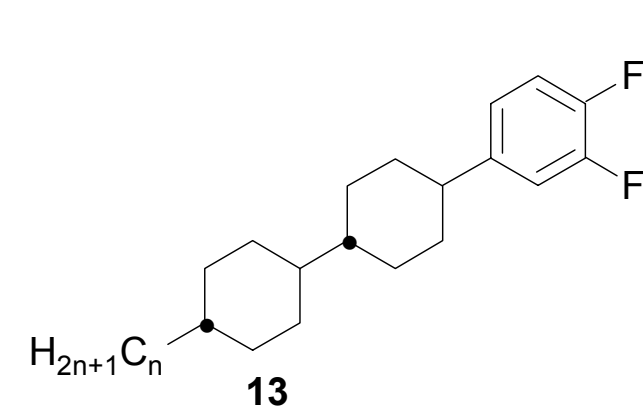

13

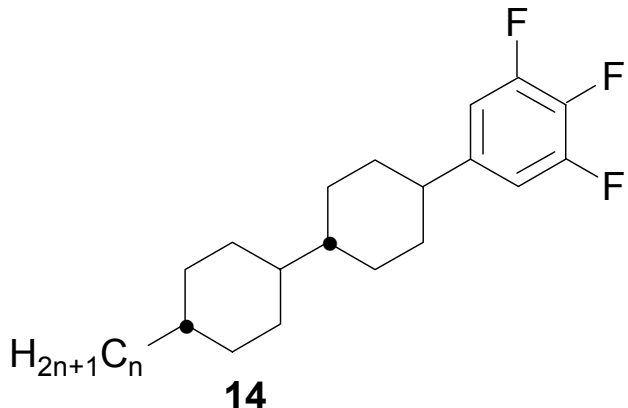

14

Abb. 5. Für TFT-LCDs entwickelte Flüssigkristalle. 
Ein "active matrix LCD" ist gesteuert durch eine Anordnung von Dünnfilmtransistoren (TFTs), von denen jeder einen Bildpunkt aktiviert. Diese Betriebsart hat den Vorteil, dass sich ein größerer Farbbereich darstellen lässt. Die Anforderungen an die chemischen Substanzen für TFT-LCDs sind nicht wesentlich verschieden von denen für einfache TN-Displays. Im besonderen benötigen sie eine gute chemische Stabilität. Die Stabilität kann indirekt als VHR (voltage holding ratio) beschrieben werden, die ein Maß für die Stabilität der Substanzen im elektrischen Feld ist. Messtechnisch wird in einer definierten Zelle mit einer flüssigkristallinen Substanzmischung zum Zeitpunkt $t=0$ ein Spannungsimpuls $V_{1}$ (für gewöhnlich $40 \mu \mathrm{s}$ ) angelegt. Nach einer bestimmten Zeit (gewöhnlich $20 \mathrm{~ms}$ ) wird gemessen, welche Spannung $\mathrm{V}_{2}$ zwischen den beiden Elektroden noch herrscht. VHR ist dann definiert als VHR: $=\mathrm{V}_{1} / \mathrm{V}_{2} \cdot 100 \%$. Substanzen mit Cyanogruppen sind nicht so geeignet dafür, weil sie im Allgemeinen niedrige VHR-Werte aufweisen, und damit einen "Ghost-effect" in den Displays auslösen. Dieser Ghost-effect bedeutet, dass auf einem LCD dargestellte Figuren nicht unmittelbar verschwinden, sondern noch für eine für das Auge deutlich wahrnehmbare Zeitspanne als Schatten sichtbar bleiben. Fluorinierte Verbindungen, besonders die 3,4Difluorophenylderivate ${ }^{[16]} 13$ und 3,4,5-Trifluorophenylderivate 14 werden als sehr geeignete Substanzen für TFT-Displays beschrieben. Diese Substanzen sind im elektrischen Feld äußerst stabil, sie besitzen eine niedrige Viskosität für schnelle Schaltzeiten, und eine hohe dielektrische Anisotropie. Die niedrige optische Anisotropie musste man in Kauf nehmen. Serien der beiden Substanzklassen 13 und 14 finden aktuell breite Anwendung in TFT-LCDs. Es wurden weitere sehr günstige Substanzen gefunden, die Chiralitätszentren in den Flügelgruppen aufweisen. Durch chirale Flügelgruppen kann die Ausbildung einer chiralen smektischen Mesophase erreicht werden. Manche dieser Substanzen weisen aufgrund ihrer chemischen Eigenschaften Ferroelektrizität auf. Mit dieser Eigenschaft kann eine extrem dünne Zelldicke $(1 \mu \mathrm{m})$ erreicht werden. Dieses System wurde von Clark und Lagerwall ${ }^{[17]}$ vorgeschlagen und wird SSFLC (surface stabilized ferroelectric liquid crystal display) genannt. Durch die Ferroelektrizität der Flüssigkristalle wird eine sehr schnelle Schaltzeit ermöglicht. Aus offenbar wirtschaftlichen Gründen hat sich im Moment aber die Konstruktion der TFT-Displays gegenüber den SSFLC-Displays durchgesetzt. Es liegt deshalb nahe zu versuchen, durch neue Ideen die Eigenschaften der in TFT-Displays derzeit verwendeten Flüssigkristalle weiter zu verbessern, und durch einige innovative Ideen neue Substanzklassen für das Betreiben von TFTs aufzufinden. 
Cyclopropanhaltige Flüssigkristalle ${ }^{[18][19]}$ sind keine Seltenheit und es wurde bislang versucht, durch den einfachen Zugang zu chiralen Cyclopropanderivaten neue nützliche Flügelgruppen für möglicherweise ferroelektrische Flüssigkristalle ${ }^{[18][19]} \mathrm{zu}$ finden. Die neue Idee in der vorliegenden Arbeit ist es, in der zentralen Einheit, dem Mittelstück eine Cyclopropyleinheit einzufügen, was hinsichtlich der hohen Elektronendichte im Cyclopropan eine vielversprechende Idee zu sein scheint. Einen schnellen Zugang zu Testsubstanzen versprach die in unserem Arbeitskreis entwickelte Kulinkovich-deMeijere-Reaktion, mit der es möglich wurde, aus Cycloalkenen diastereoselektiv trans-substituierte Cyclopropylamine herzustellen. Parallel zur historschen Entwicklung flüssigkristalliner Substanzen entstand die Idee, als erste Substanzgruppe Schiff'sche Basen zu synthetisieren, um zu testen, ob sich eine weitere Entwicklung von Substanzen mit zentraler Bicyclo[3.1.0]hexan-Einheit lohne.

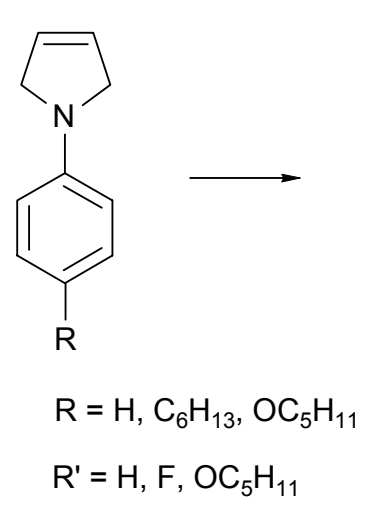

15

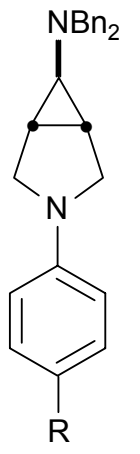

16

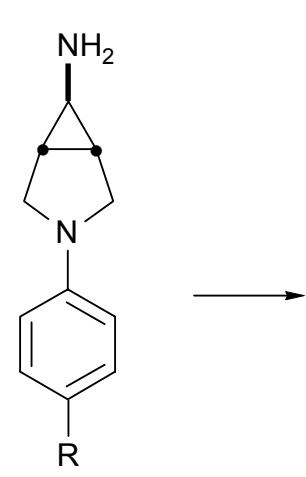

17

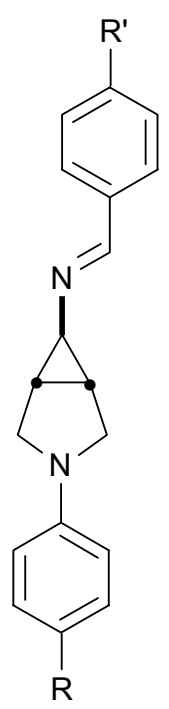

18

Schema 1. Synthese der ersten Substanzklasse mit zentraler 3-Aza-bicyclo[3.1.0]hexanEinheit.

Im Jahr 2000 wurden im Arbeitskreis de Meijere einige Beispielsubstanzen für 3-Phenyl-6exo-(N-benzylidenamino)-3-azabicyclo[3.1.0.] hexanderivate ${ }^{[20]} \mathbf{1 8}$ mittels Kulinkovichde Meijere-Reaktion ${ }^{[21]}$ hergestellt und auf ihre physikalischen Eigenschaften untersucht. Die Substanzen zeigten flüssigkristallinen Charakter und hinsichtlich ihrer Dipolmomente, optischen Anisotropie und Übergangstemperaturen vielversprechende Eigenschaften im Vergleich mit ähnlichen bekannten Substanzen. 
Tabelle 1. Vergleich der Übergangstemperaturen von 19 und einer ähnlichen Substanz

Substanz

In einem Vergleich mit der analogen Verbindung 20 ohne zentrale Azabicyclo[3.1.0]hexanEinheit stellte sich heraus, dass die gemessene Temperatur für den N-I-Übergang um $80{ }^{\circ} \mathrm{C}$ niedriger liegt als die der bekannten Verbindung. Ein Nachteil der neuen Substanz ist neben der bekannten chemischen Labilität die geringe Löslichkeit der Aldimine in bekannten Basismischungen wie z. B. ZLI-1132.

Die Geometrie der neuen Molekülklasse wurde per MOPAC/AM1-Berechnung bestimmt, um dann Stuktur-Eigenschafts-Betrachtungen anzustellen. In Abb. 6 ist erkennbar, dass der an die Iminofunktion gebundene Aromat sich um $63^{\circ}$ aus der Molekülebene herausdreht. Weiterhin wurde berechnet, dass sich die Bindungen an den beiden Stickstoffatomen um $21^{\circ}$ aus der Molekülebene herausheben. Da in dieser Substanzklasse eine Voraussetzung für gute flüssigkristalline Eigenschaften eine möglichst hohe Planarität ist, wurden die nachteiligen Eigenschaften durch weitere Molekül-Modellierungen zu verbessern versucht. 


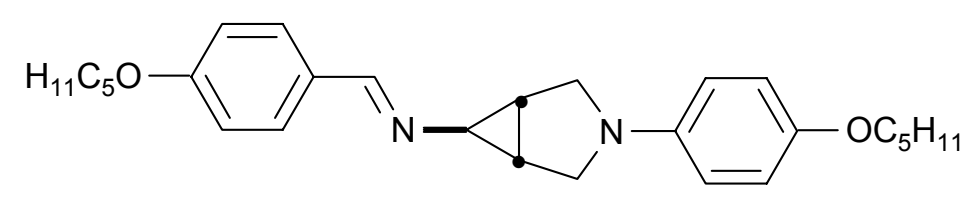

19
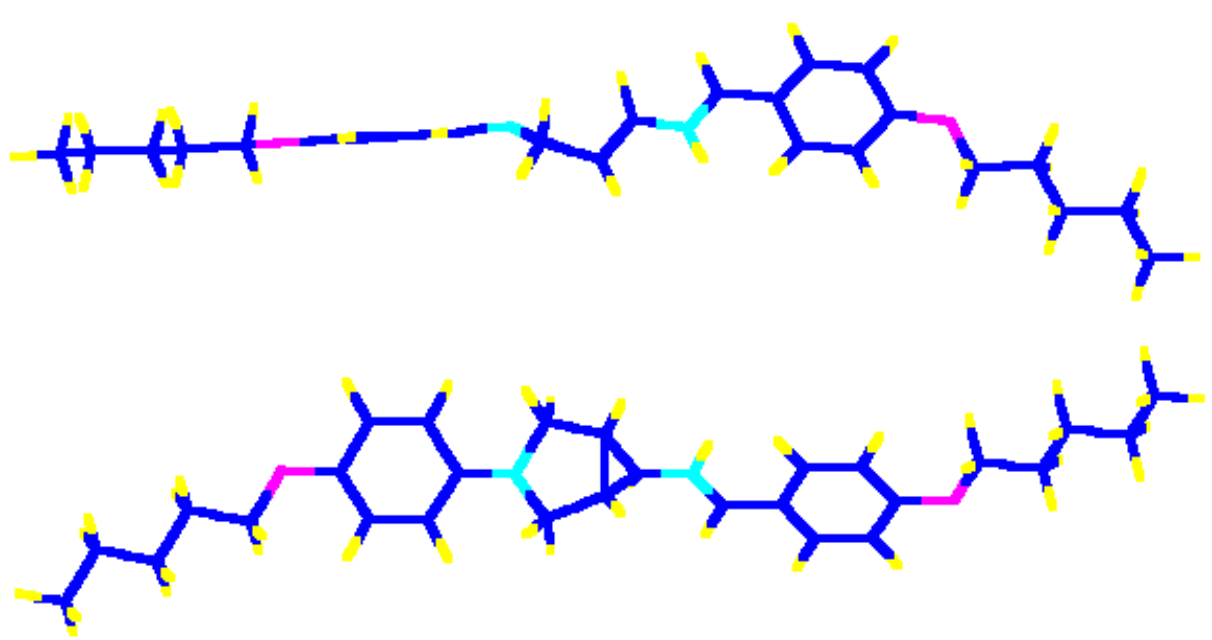

Abb. 6. Berechnete Molekülstruktur des 3-(4-Pentyloxyphenyl)-6-exo-[N-(4-pentyloxybenzylidenamino)-3-azabicyclo[3.1.0]hexans 19.

Daraus wurde ein Strukturvorschlag erarbeitet, in dem die beiden Substituenten in den 3- und 6- Positionen sich weniger aus der Molekülebene herausdrehen. Dabei bieten sich unter geometrischen Aspekten für die Substituenten an der Bicyclo[3.1.0]hexan-Einheit sowohl Aromaten, als auch $n$-Alkyl und Cycloalkylgruppen an. 

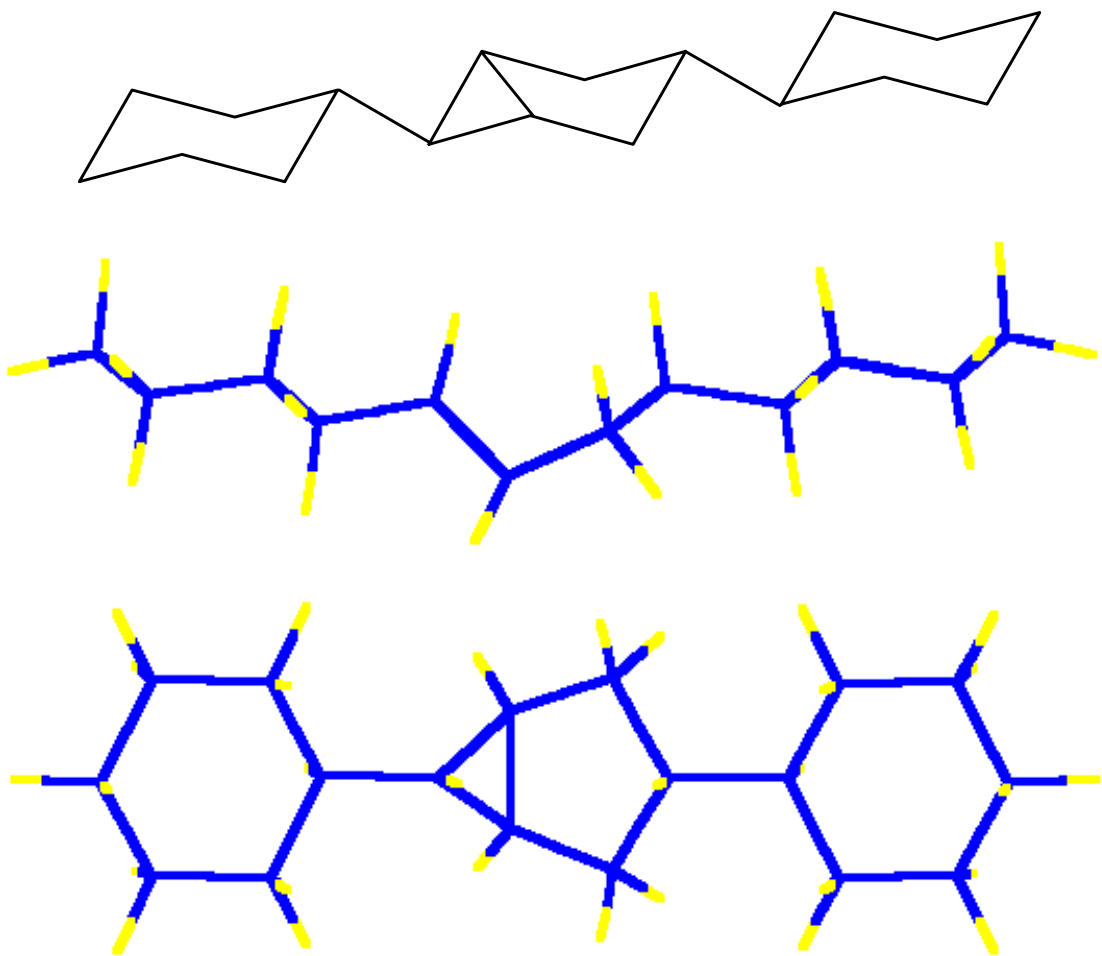

Abb. 7. Durch MOPAC/AM1 berechnete neue Zielverbindungen.

\section{Zielsetzung}

Um mehr über die Eigenschaften der neuen Bicyclo[3.1.0]hexane zu erfahren, ist es nötig, einige isomorphe, mit bekannten Molekülen vergleichbare Substanzen herzustellen. Dazu sollen die Substituenten in den 3- und 6-Positionen variiert werden. Mögliche Substituenten sind Ester, substituierte Aryle, Alkyle oder 4-substituierte Cycloalkyle. Da bislang mesogene Substanzen mit zentraler Bicyclo[3.1.0]hexan-Einheit unbekannt sind, müssen Wege zur Synthese dieser neuen Substanzklasse entwickelt werden. 


\section{B. Hauptteil}

\section{Allgemeine Vorüberlegungen}

Die mittels der Kulinkovich-de Meijere-Reaktion synthetisierten Azabicyclo[3.1.0]hexane stellen sich als eine sehr günstige gelungene Synthese dar, weil es mit dieser Reaktion möglich ist, diastereoselektiv die exo-konfigurierten Azabicyclo[3.1.0]hexylamine 25 in guten Ausbeuten herzustellen. Dabei wird aus dem 1,4-cis-But-2-en-diol 21 in guter Ausbeute das entsprechende Dimesylat 22 hergestellt, das mit einem primären Amin das 3,4Dehydropyrrolidin 23 bildet. Dieses kann mittels titanvermittelter Cyclopropanierung mit Ligandenaustauschreaktion in ein 6-exo-Dibenzylamino-3-azabicyclo[3.1.0]hexan überführt werden $^{[20]}$. Die beiden Benzylgruppen können unter reduzierenden Bedingungen entfernt werden. Das resultierende freie primäre Amin kann mit Aldehyden zu den Aldiminen 25 kondensiert werden, die strukturelle Ähnlichkeit mit bekannten mesogenen Substanzen der Form 20 aufweisen.

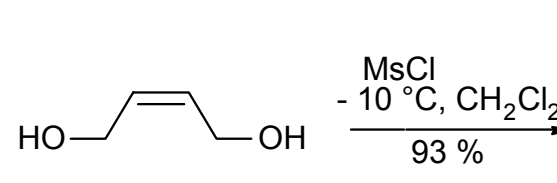

21

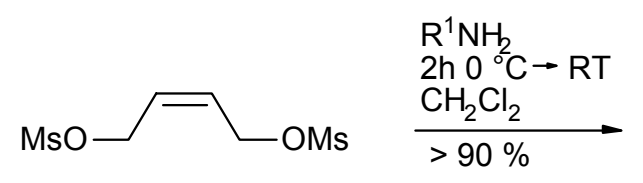

22

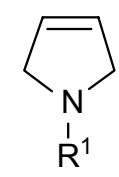

23

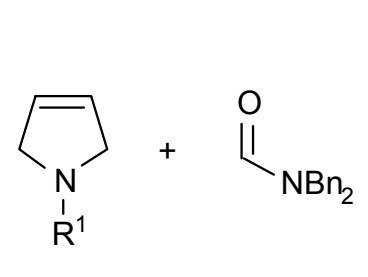

24
1.) $\mathrm{MeTi}(\mathrm{O} i \mathrm{Pr})_{3}$

2.) $\mathrm{CyHexMgBr}$

3.) $\mathrm{H}_{2} / \mathrm{Pd} / \mathrm{C}, \mathrm{HOAC}$

$\frac{\text { 4.) } \mathrm{R}^{2} \mathrm{CHO}}{>50 \%}$ $>50 \%$

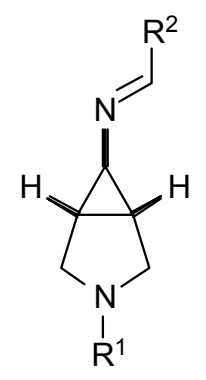

25

Schema 2. Synthese der 3-Aryl-6-exo-benzylylidenamino-3-azabicyclo[3.1.0]hexane 25.

Durch eine andere Variante der Kulinkovich-Reaktion ${ }^{[22]}$ sind diastereoselektiv transCyclopropanole darstellbar. Diese wären entweder in einer Ether- oder in einer Estersynthese als Kupplungsgruppe nutzbar. Wegen der allgemeinen Instabilität dieser Strukturen muss aber für die Herstellung der Zielstrukturen vom Typ 26 ein anderer Syntheseweg gewählt werden. Die Synthese von zu 25 analogen Substanzen ohne den Stickstoffsubstituenten in 6-Position kann somit mit den bislang zur Verfügung stehenden Reaktionen nur durch Addition eines 
Carbens oder Carbenoids ermöglicht werden.

Eine zu der Kulinkovich-de Meijere analoge Reaktion mit vergleichbarer Selektivität konnte nicht aufgefunden werden. Da darüber hinaus der Stickstoff in Position 3 im Ring durch die isoelektronische $\mathrm{CH}$-Gruppierung ersetzt werden soll, muss der Aufbau des Rinsystems von Anfang an modifiziert werden.

Es sind viele verschiedene Kupplungsgruppen bekannt. Eine der einfachsten Methoden zur Kupplung zweier Molekülfragmente ist die Verknüpfung mittels Esterfunktionalität. Da bei der Synthese von mesogenen Substanzen die Vermeidung von Funktionalitäten günstig ist, können bei anspruchsvolleren Synthesen die Kupplungsgruppen $\mathrm{K}^{\mathrm{X}}$ einfach eine $\sigma$ Bindungen sein. Für die Wahl der Reste steht prinizipiell das gesamte bei der Synthese von Flüssigkristallen verfügbare Repertoire zur Wahl. Es müssen einige geeignete Substanzen davon ausgewählt werden. Neben der Verfügbarkeit der Edukte wird als Auswahlkriterium zeitweise auch ,atom efficiency“ und wegen möglicher HPLC-Trennung mit UV-Detektion die Beinhaltung chromophorer Gruppen in Betracht gezogen.
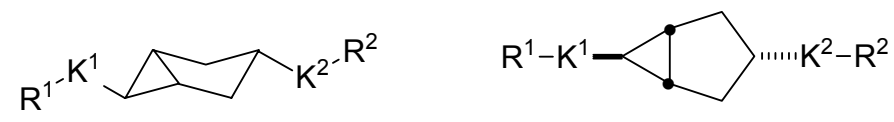

$\mathrm{K}^{1}, \mathrm{~K}^{2}=$ Kupplungsgruppen

$\mathrm{R}^{1}, \mathrm{R}^{2}=$ nAlkyl-, Cycloalkyl- oder Arylreste

26

Abb. 8. Bicyclo[3.1.0]hexanderivate als Zielstrukturen. 


\section{Synthese der Diester}

\subsection{Retrosynthese}

In Analogie zu der historischen Entwicklung der Flüssigkristalle liegt auch hier der Versuch nahe, durch den einfachen Zugang zu Estern zuerst diese Klasse zu synthetisieren um sie hinsichtlich ihrer physikalischen Eigenschaften untersuchen $\mathrm{zu}$ können. Mittels der umfangreichen Datensammlung LiqCryst $4.3^{[23]}$ können die synthetisierten Verbindungen mit bereits bekannten Substanzen verglichen werden. Es werden Carboxylgruppen in den Positionen 3 und 6 des Bicyclo[3.1.0]hexans benötigt, die mit Hydroxyfunktionalitäten eines geeigneten Alkohols zu dem Diester 35 gekuppelt werden können. Der entsprechende Diester 33 sollte zugänglich sein durch Addition von Ethyldiazoacetat an die zu dem 3,4-Dehydropyrrolidin 24 analogen Cyclopenten-4-carbonsäureester 32. Die tertbutylgeschützte Säure 32 sollte sich einfach durch säurekatalysierte Addition von Isobuten an die Carbonsäure $\mathbf{3 1}$ herstellen lassen.
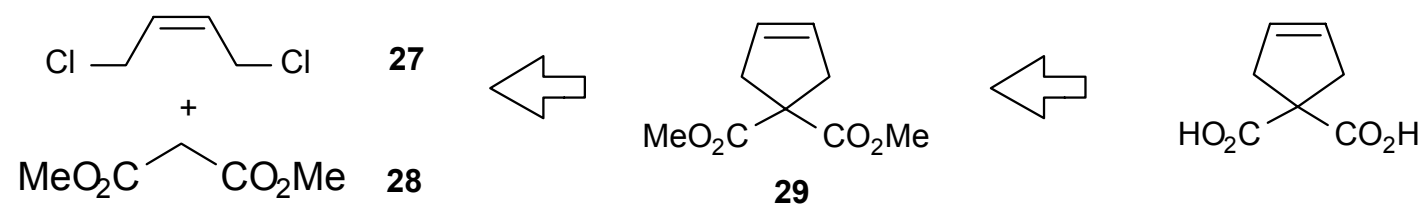

30<smiles>O=C(O)C1CC=CC1</smiles>

31

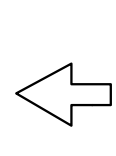

33

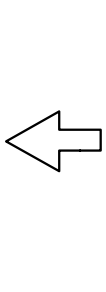

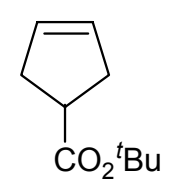<smiles>C1=CCCC1</smiles>

32
$\mathrm{C}_{2}{ }^{t} \mathrm{Bu}$

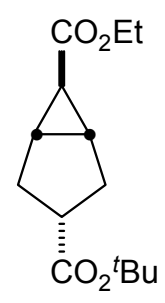

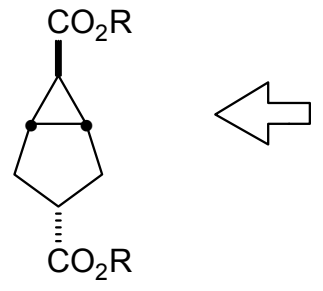

35

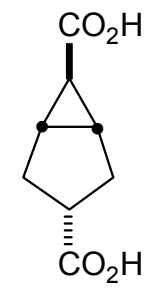

34

Schema 3. Retrosynthese der Zielstruktur 35.

Die Synthesen der Substanzen 27 - 31 sind literaturbekannt ${ }^{[24]}$. Cyclopent-3-encarbonsäure wurde schon um 1960 für die Synthese pharmakophorer Substanzen ${ }^{[25]}$ als Edukt benötigt. 
Die Synthese dieser Säure machte aber über mehr als 20 Jahre große Probleme. In den Arbeitskreisen von Deprés und Greene ${ }^{[24]}$ konnte durch Modifikation der Lösungsmittel, der Basen und der Reste die Ausbeuten der früheren Vorschrift für die Malonestersynthese nach Murdock und Angier ${ }^{[26]}$ von $19-33 \%$ auf $75 \%$ gesteigert werden. Deprés und Angier fanden heraus, dass neben der erwünschten Cyclopent-3-en-1,1-dicarbonsäuredimethylester das unerwünschte Vinylcyclopropan 36 im Verhältnis von ca. 6:4 gebildet wird. Es wurde weiter herausgefunden, dass harte Kationen und der Übergang zu aprotischen Lösungsmitteln sowie der Einsatz der Malonester kleiner Alkohole die Ausbeute an der erwünschten Säure erhöht. Die letztlich benutzte Synthese des Diesters mit nachfolgender Verseifung liefert in einer „Eintopfreaktion“ die Disäure 30 mit 92\% Ausbeute ${ }^{[24]}$. Nachfolgende Decarboxylierung stellt die benötigte Cyclopent-3-encarbonsäure mit $89 \%$ (82\% über 3 Stufen) Ausbeute ${ }^{[24]}$ zur Verfügung. Das als Ausgangssubstanz benötigte cis-1,4-But-2-endichlorid 28 ist zwar kommerziell erhältlich lässt sich aber in einer Reaktion des cis-1,4-But-2-endiols sehr preisgünstig in akzeptablen Ausbeuten selbst herstellen.

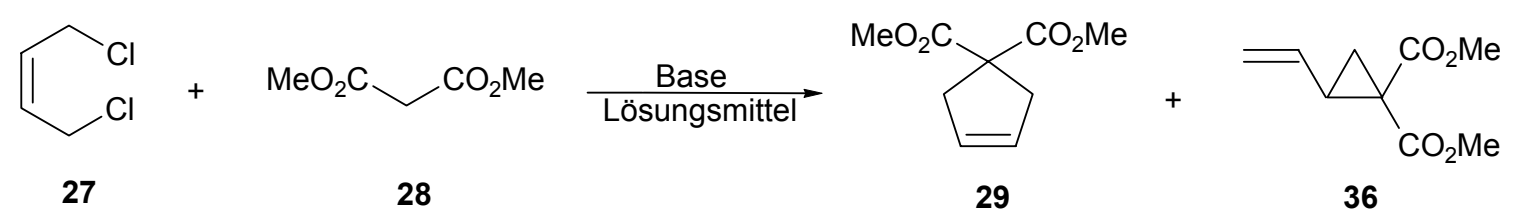

Schema 4. Darstellung des Cyclopent-3-en-1,1-dicarbonsäuremethylesters 29. 


\subsection{Darstellung der Ausgangsmaterialien}

Aus wirtschaftlichen Gründen wurde aus einer Lösung von cis-But-2-en-1,4-diol 21 in Pyridin mit Thionylchlorid das entsprechende cis-But-2-en-1,4-dichlorid 27 synthetisiert ${ }^{[27]}$. Dabei verläuft die Reaktion sehr heftig, und für die Hydrolyse der Reaktionsmischung muss auf einen sehr gut arbeitenden Abzug zurückgegriffen werden. Es ist hier anzumerken, dass sich die Reaktionsansätze für Labormaßstäbe beliebig vergrößern lassen, allerdings bei Reaktionen mit 3 Mol des Diols als Edukt die Zugabe des Thionylchlorids lange dauert, weil sich die Temperatur der Reaktionsmischung während der Zugabe des Thionylchlorids mit einem Eis-Kochsalz-Kühlbad nicht ausreichend kontrollieren lässt und Trockeneis oder flüssiger Stickstoff als Kühlmittel zu anderen apparativen Problemen führen.

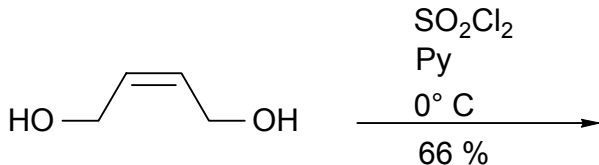

21

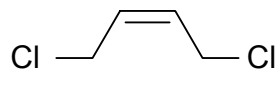

27

Schema 5. Laborsynthese des 1,4-Dichlor-cis-But-2-en 27.

Die nachfolgende Malonestersynthese mit Lithiumhydrid als Base liefert literaturgemäß ${ }^{[24]}$ gute Ausbeuten und die Verseifung des Cyclopent-3-endicarbonsäuredimethylesters 29 kann als zweiter Schritt ohne Isolierung des Diesters durchgeführt werden. Die Disäure 30 kann als bei Raumtemperatur stabile Substanz isoliert werden. Thermische Decarboxylierung liefert in guter Ausbeute die Cyclopenten-3-carbonsäure 31, die in einer Ampulle mit Isobuten und einer katalytischen Menge Schwefelsäure in den tertButylester 32 überführt werden kann. Mit dieser Reaktionssequenz kann das für folgende Synthesen benötigte Edukt 31 in 100 g-Mengen zur Verfügung gestellt werden. 

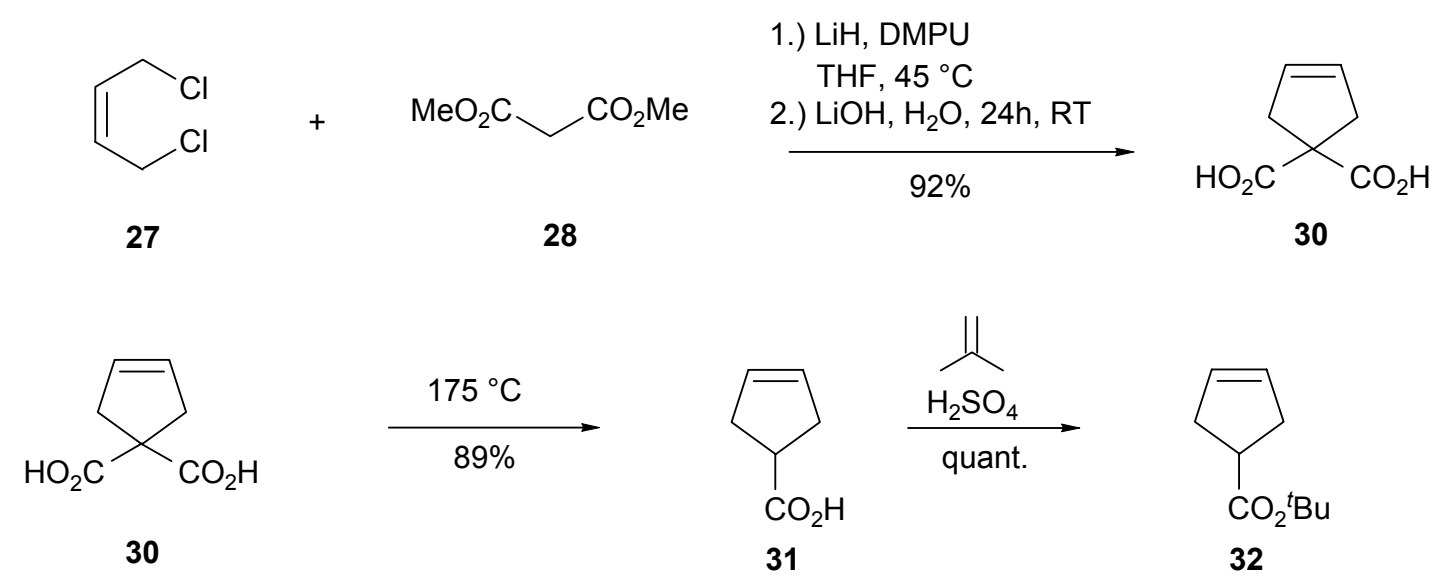

Schema 6. Synthese des Cyclopent-3-encarbonsäure-tertbutylesters 32.

Die Addition von dem nach Searle ${ }^{[28]}$ synthetisierten Ethyldiazoacetat an olefinische Doppelbindungen ist schon seit langem bekannt ${ }^{[29]}$. Diazoverbindungen zerfallen bei Behandlung mit vielen Übergangsmetallsalzen unter Stickstoffabstpaltung in die entsprechenden Carbenoide. Es gibt viele verschiedene Übergangsmetallsalze, die diese Reaktion katalysieren ${ }^{[30]}$, die bekanntesten darunter sind dimeres Rhodiumacetat, Palladiumacetat und Kupferacetylacetonat.

Bei der hier benötigten Reaktion wurden beste Ausbeuten und wenig Dimerisierungsprodukt mit dimerem Rhodiumacetat erhalten ${ }^{[30]}$. Glücklicherweise ist das Additionsprodukt des Carbenoids an ein Cyclopentenderivate ein Gemisch aus nur 2 Diastereomeren von 4 möglichen. Vermutlich wegen sterisch zu großer Nachteile entstehen die beiden denkbaren 3endo-Diastereomere nicht. Die als Nebenprodukte entstandenen Das als Verunreinigung entstandene Diethylfumarat kann durch Kugelrohrdestillation entfernt werden. Die beiden Diastereomere 33a und 33b sind chromatographisch trennbar.<smiles>CCOC(=O)C=[W]</smiles>

37<smiles>CCOC(=O)C1CC=CC1</smiles>

32

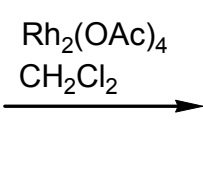<smiles>CCOC(=O)C1C[C@H](C(=O)OCC)[C@H](C(=O)OCC)C1</smiles>

$33 b$

$$
\text { ds }=7: 3
$$

Schema 7. Addition von Ethyldiazoacetat 37 an Cyclopent-3-encarbonsäure-tertbutylester 32.

Der Versuch aus 33a selektiv die Monosäure 36 freizusetzen scheiterte, obwohl eine ähnliche Versuchsvorschrift ${ }^{[31]}$ bekannt ist, um Methylester selektiv in Gegenwart von tertButylestern 
zu spalten. Eine Spaltung des tertButylesters unter Erhalt des Methylesters mit nachfolgender Veresterung würde nicht zum Ziel führen, weil danach ein Ester eines primären Alkohols selektiv in Anwesenheit eines Esters sekundärer Alkohole zu spalten wäre. Ein Verfahren zur Lösung dieser Aufgabe ist in der Literatur nicht bekannt. Unter literaturbekannten basischen Bedingungen sollte die Isomerisierung des Diastereomerengemisches 33 möglich sein. Trotz Variation der Lösungsmittel ( $\mathrm{Et}_{2} \mathrm{O}, \mathrm{THF}$, Benzol, Toluol) und der verwendeten Basen $\left(\mathrm{NEt}_{3}\right.$, $\mathrm{K}_{2} \mathrm{CO}_{3}, \mathrm{KO}{ }^{t} \mathrm{Bu}$ ) trat die erwartete Isomerisierung $\mathrm{zu}$ dem thermodynamisch stabileren 33a nicht ein. Statt der erwarteten Isomerisierung tritt aber die Verseifung des Diesters in die freie Disäure 34a ein.

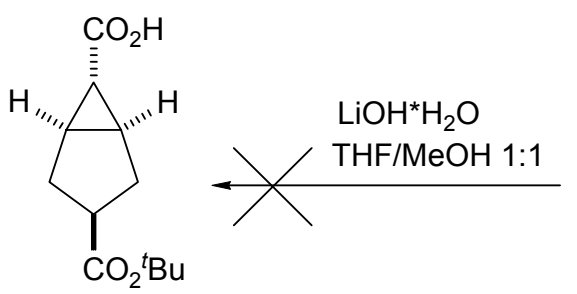

36<smiles>CCOC(=O)C1C[C@@H]2C(C(=O)OCC)[C@H]2C1</smiles>

$33 a$
$\mathrm{LiOH}^{*} \mathrm{H}_{2} \mathrm{O}$ $\mathrm{THF} / \mathrm{MeOH} 1: 1$
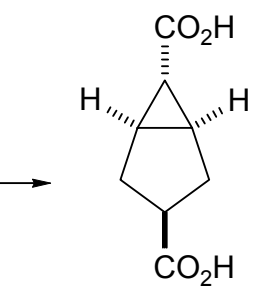

$34 \mathrm{a}$

Schema 8. Versuch zur selektiven Entschützung von Ethylester in Gegenwart des tert-Butylesters. 
2.3 Synthese der Bicyclo[3.1.0]hexan-3,6-dicarbonsäurediester

Aus der freien Disäure 34a lassen sich mit der DCC-Methode nach Neisius und Steglich ${ }^{[32]}$ in guter Ausbeute mit substituierten Phenolen vom Typ 38 die Diester 35 synthetisieren. Das Produkt lässt sich gut säulenchromatographisch reinigen. Die Struktur von 35a wurde mittels Röntgenbeugung bestimmt und das Zielprodukt als erwünschte Testsubstanz auf seine physikalischen Eigenschaften untersucht.

Es wurde weiterhin versucht, 4-substituierte Cyclohexanole mit der Disäure 34a zu kuppeln. Dieses Experiment stellte sich aber als ungeschickt heraus, da diese Zielprodukte über mehr als 20 aliphatische Kohlenstoffatome verfügen, was eine Analyse mittels NMR-Spektroskopie vereitelt und eine Kristallisation für eine Kristallstrukturanalyse mittels Röntgenbeugung aufgrund des paraffinartigen Charakters der Substanzen nicht gelang.
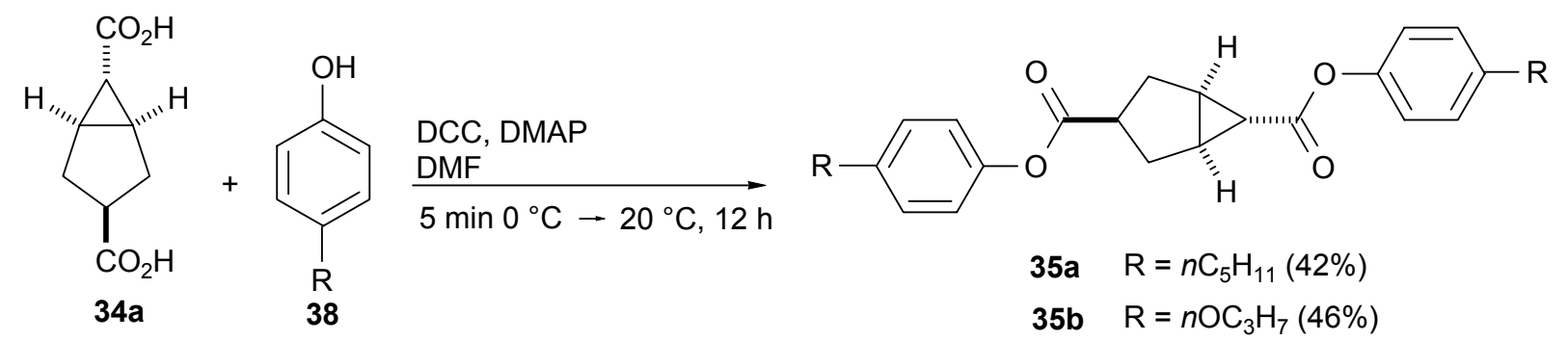

Schema 9. Synthese der Bicyclo[3.1.0]hexan-3,6-dicarbonsäurediester 35. 


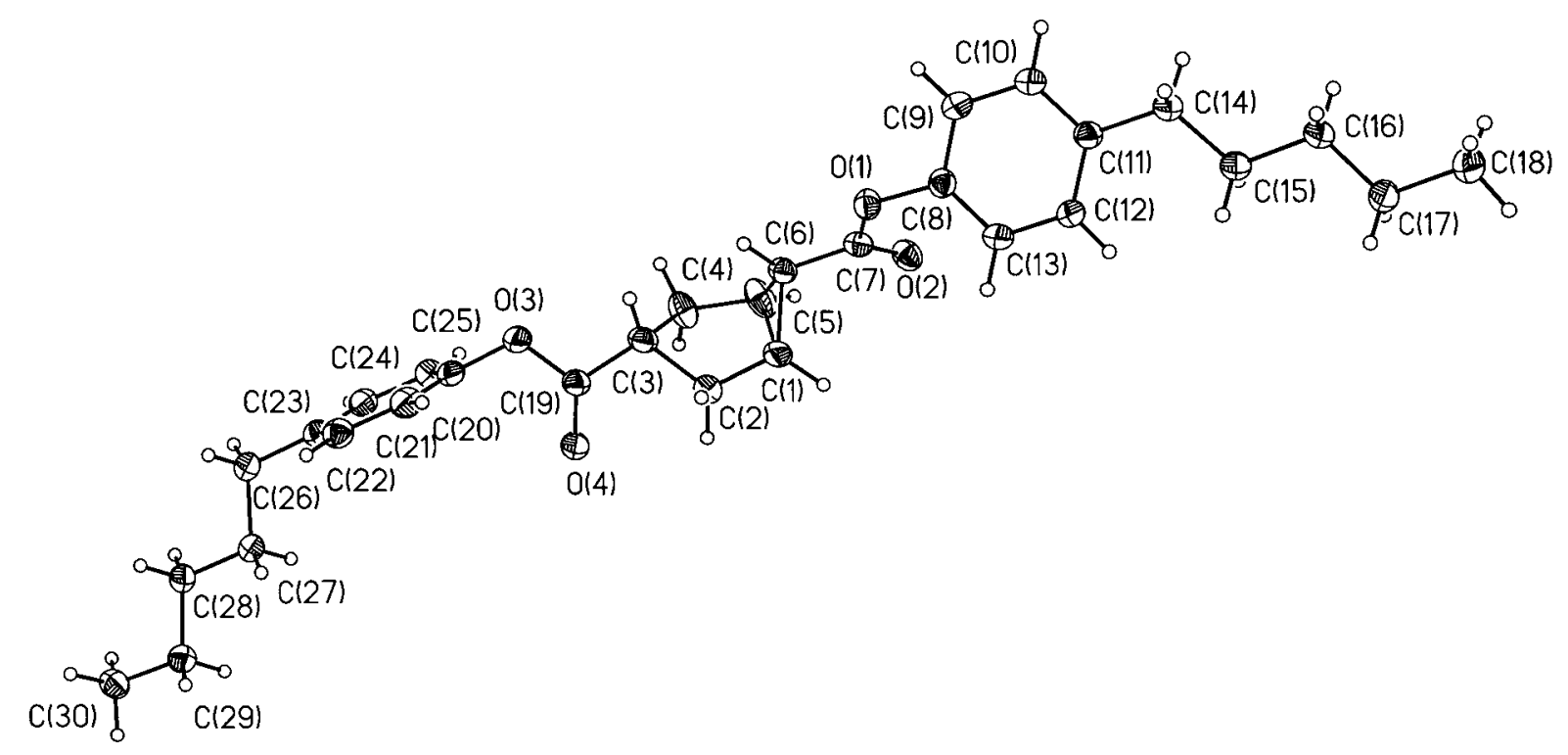

Abb. 9.Struktur der Verbindung 35a im Kristall. 
2.4 Diskussion der Eigenschaften von exo,exo-Bicyclo[3.1.0]hexan-3,6-dicarbonsäure-3,6di(4-n-pentylphenyl)ester

Mit dem Diester 35a liegt zum ersten mal eine stickstofffreie Substanz mit flüssigkristallinen Eigenschaften und zentraler Bicyclo[3.1.0]hexan-Einheit vor. Der Vergleich der physikalischen Eigenschaften dieser Verbindung gibt Aufschluss über die Attraktivität dieser neuen Idee. Da absolute Aussagen über die Güte eines neuen Moleküls schwer möglich sind, ist ein Vergleich mit einer ähnlichen Substanz nötig. Als eine solche Substanz bietet sich 39 an, die zu experimentellen Zwecken in den Laboratorien der Fa. Chisso Petrochemicals Co. literaturgemäß ${ }^{[33]}$ synthetisiert wurde.

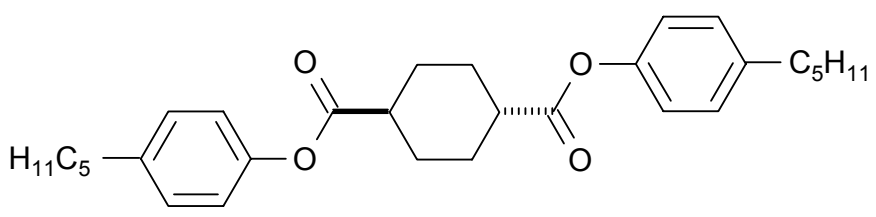

39

Abb.10. Diphenyl-1,4-cyclohexanedicarboxylate 39 als Vergleichssubstanz.

Aus Tabelle 1 ist ersichtlich, dass 35a viele Vorteile gegenüber der bekannten Substanz 39 aufweist. Ein wichtiger Vorteil ist die Mischbarkeit mit der flüssigkristallinen Basismischung. Für gewöhnlich wird als solche Mischung die käufliche Basismischung Merck ZLI-1132 gewählt. 35a zeigt günstigerweise im Gegensatz zu 39 nur einen einzigen Phasenübergang. Dies ist ein Vorteil für die Beherrschung der Pixelsteuerung in den anfangs genannten Displays. Der Phasenübergang liegt um $70.20{ }^{\circ} \mathrm{C}$ niedriger als der der bekannten Vergleichssubstanz. In den verschiedenen Temperaturbereichen erstreckt sich die nematische Phase beider Komponenten über ca. $25^{\circ} \mathrm{C}$, wobei die neue Substanz 35a einen um $1.40^{\circ} \mathrm{C}$ breiteren Temperaturbereich für die nematische Mesophase aufweist. 
Tabelle 1. Vergleich physikalischer Eigenschaften der Verbindungen 35a und 39.

\begin{tabular}{lcc}
\hline Substanz & 35a & 39 \\
\hline Phasenübergänge $\left[{ }^{\circ} \mathrm{C}\right]$ & $\mathrm{C} 60.84 \mathrm{~N} 86.43 \mathrm{I}$ & $\mathrm{C} 95.09\left(\mathrm{~S}_{\mathrm{B}} 93.64\right) \mathrm{S}_{\mathrm{A}} 131.13 \mathrm{~N}$ \\
& & $155.32 \mathrm{I}$ \\
Dielektrische Anisotropie $\Delta \varepsilon$ & $0.5^{\mathrm{a})}$ & $-1.1^{\mathrm{b})}$ \\
Optische Anisotropie $\Delta n$ & $0.070^{\mathrm{a})}$ & $0.117^{\mathrm{b})}$ \\
Mischbarkeit in Basismischung & $>15 \%$ & $5 \%$ \\
\hline
\end{tabular}

a) Extrapolierter Wert: 15\% in der Basismischung

b) Extrapolierter Wert: $5 \%$ in der Basismischung 


\section{Synthesewege für 3,6-disubstituierte Bicyclo[3.1.0]hexanderivate als Mittelstück}

In der historischen Entwicklung flüssigkristalliner Substanzen wurde erkannt, dass die Eigenschaften mesogener Substanzen umso günstiger sind, je weniger Funktionalitäten im Molekül vorhanden sind. Die Bemühungen im Rahmen dieser Synthesen haben die Zielsetzung, das Bicyclo[3.1.0]hexanmittelstück ohne die üblichen Kupplungsgruppen an den Positionen 3 und 6 mit $n$-Alkyl-, Cycloalkyl- oder Arylresten zu verbinden. Daraus ergeben sich mehrere mögliche Substanzklassen.

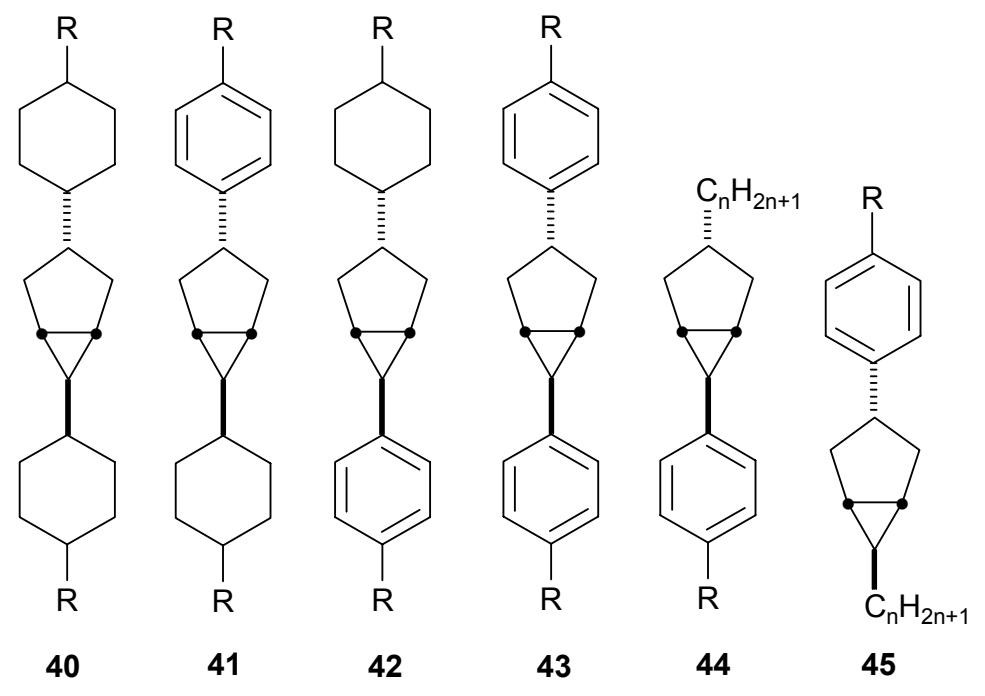

Abb. 11. Mögliche Substanzklassen zur weiteren Synthese.

Aus den bei der Synthese der Diester gesammelten Erfahrungen bezüglich der Analyse reiner gesättigter Kohlenwaserstoffe wurde beschlossen, die Synthese der Substanzklasse 40 zu vernachlässigen. Die Charakterisierung der Zielmoleküle wäre äußerst aufwendig, da diese Substanzen mit paraffinartigem Charakter vermutlich keine zur Röntgenstrukturanalyse geeigneten Kristalle bilden. Auch die Analyse der Substanzen mittels ${ }^{1} \mathrm{H}$ - oder ${ }^{13} \mathrm{C}-\mathrm{NMR}$ Spektroskopie könnte keine Aufschlüsse über die Konfiguration der sechs Stereozentren geben. Die Isomerentrennung mittels HPLC würde wegen fehlender chromophorer Gruppen nicht möglich sein. Selbst bei erfolgreicher Synthese einer reinen Substanz fiele es schwer, zu bestimmen um welches Isomere es sich handelt.

Für die Synthese der Substanzklassen 41 - 45 müssen Synhtesewege entwickelt werden, um Kupplungen an den Positionen 3 und 6 am Bicyclo[3.1.0]hexan zu ermöglichen. Da $n$ Alkyl-, Cycloalkyl- und Arylsubstituenten in diesen Positionen ermöglicht werden sollten, nimmt die synthetische Aufgabenstellung sowohl den Charakter konvergenter Synthesen, als auch den 
kombinatorischer Chemie an. Es ist deshalb von Anfang an klar, dass bei erfolgreichem Auffinden von einigen Kupplungsreaktionen nur exemplarisch einige Molekülstrukturen synthetisiert werden können, um die Leistungsfähigkeit der Moleküle hinsichtlich ihrer technischen Anwendung zu analysieren.

Chemisch bieten sich nur wenige Möglichkeiten, ein Bicyclo[3.1.0]hexangerüst aufzubauen. In dem vorliegenden Fall erscheinen einige sinnvolle Methoden zu einer erfolgreichen Synthese von 3,6-disubstituierten Bicyclo[3.1.0]hexanen: Als erstes muss die Synthese des Bicyclo[3.1.0]hexangerüstes mittels intramolekularer Variante via Acetoacetatsynthese ${ }^{[34]}$ an Zimtalkoholenderivate mit nachfolgender Diazotransferreaktion ${ }^{[35]}$ ausprobiet werden. Eine ebenfalls denkbare Methode, die eine gewisse Analogie zur titanvermittelten Synthese von Cyclopropylaminen darstellt, ist die Verwendung von Fischer-Carbenkomplexen. Literaturbekannt ist die Synthese von Arylcyclopropanen mittels Eisen- ${ }^{[36]}$ und Wolframcarbenkomplexen $^{[37]} 46$ und 49. Wegen des großen sterischen Anspruchs der Liganden wird mit diesen Fischer-Carbenen das unerwünschte thermodynamisch instabilere endo-Produkt in großem Überschuss und mit mäßiger Ausbeute gebildet.
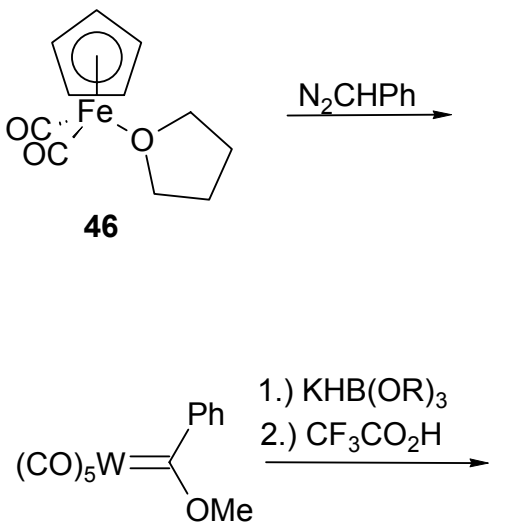

49
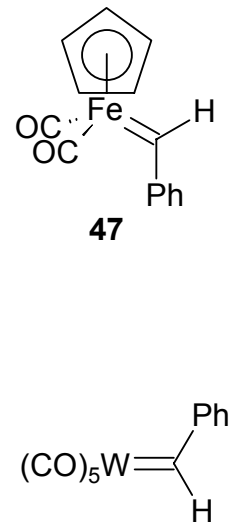

50
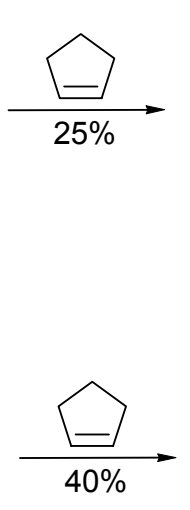

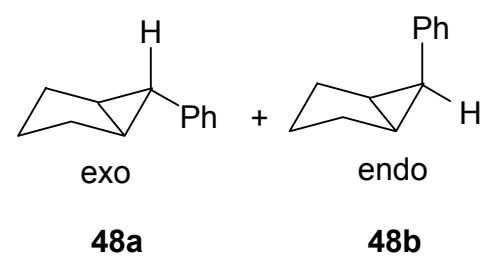

endo:exo 97:1

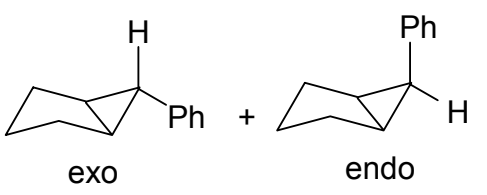

$48 a$ endo:exo 2.6:1

Schema 10. Synthese von 6-Arylbicyclo[3.1.0]hexanen über Fischer-Carbene.

Eine weitere Variante ist eine Addition eines aus Aryldichlormethan durch Einwirkung von Kalium-tertbutanolat ${ }^{[38][39][40]}$ oder Methyllithium synthetisieren Chlorcarbens an 4substituierte Cyclopentenderivate mit nachfolgender Dehalogenierung.

Schließlich ist als letzte Methode eine Addition von aus Diazoverbindungen mittels Übergangsmetallkatalyse oder photolytisch erzeugten Carbenen an die Cyclopentenderivate in Betracht zu ziehen.

Die intramolekulare Variante ist wegen der thermodynamisch begünstigten Bildung nur eines 
von vier denkbaren Produkten, die Chlorcarben- und übergangsmetallkatalysierte oder photolytische Variante wegen der vermutlich leichten Variierbarkeit der Edukte interessant.

Die synthetischen Bemühungen müssen sich deshalb zum einen auf die Auffindung geeigneter Edukte für die intramolekulare Variante sowie die Synthese von 4-substituierten Cyclopentenderivaten konzentrieren.

Die Synthese geeigneter 4-substituierter Cyclopentenderivate, die nach einer erfolgreichen Carbenaddition gewünschte Moleküle mit flüssigkristallinen Eigenschaften liefern könnten, ist wegen der schon erwähnten leichten Modifizierbarkeit der Edukte sehr interessant. Als Edukte für solche Reaktionen bieten sich Cyclopent-4-encarbonsäure 31 und 4-Cyclopentenol 80 an. 


\section{Retrosynthetische Überlegungen zu Bicyclo[3.1.0]hexanderivaten}

Gemäss üblicher Retrosynthesen ergeben sich für die Synthese von substituierten Bicyclo[3.1.0]hexanen mehrere Schnittmöglichkeiten. Daraus resultieren verschiedene Edukte mit einigen synthetischen Besonderheiten. Gemäß Schema 11 muss zu Anfang ein Arylacetoacetat 52 synthetisiert werden. Ist die Herstellung des Acetoacetats gelungen, sollte eine Retro-Claisen-Reaktion mit nachfolgender Diazotransferreaktion mittels Tosylazid gute Resultate bringen. Die entstandene Diazoverbindung 53 sollte in guter Ausbeute zur intramolekularen Addition ${ }^{[41]}$ eines Carbenoids fähig sein. Ringöffnung des Lactons 54 sollte ein Diol liefern, dessen Folgeprodukte mögliche Reaktionsvorläufer für eine Ringschlußreaktion bieten sollten.<smiles>[R]C1CC2C([R])C2C1</smiles>

57

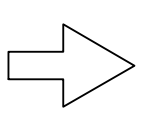

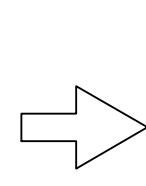<smiles>[R]C=CCOC(=O)C=N</smiles>

53

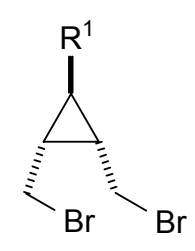

56
.<smiles>C1CCCCC1</smiles>

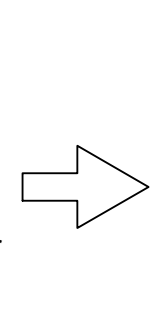

(25)

52

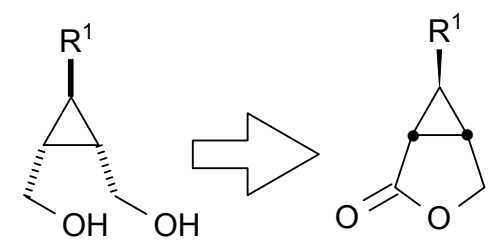

54<smiles>C1=CCCC1</smiles><smiles>[R]C=CCO</smiles>

51

Schema 11. Retrosynthese eines 3,6-disubstituierten bicyclo[3.1.0]hexans 57. 
4.1 Die Synthese von 2-Oxo-6-phenyl-3-oxabicyclo[3.1.0]hexan

M. P. Doyle ${ }^{[42]}$ et al. haben ohne weitere experimentelle Details die Synthese von 2-Oxo-6phenyl-3-oxabicyclo[3.1.0]hexan (54) veröffentlicht. Deshalb wurden andere Wege zur Synthese des Lactons 54 gefunden werden. Das Cinnamylacetoacetat 52 läßt sich aus Zimtalkohol und Diketen gewinnen. Die Tributylphosphin-katalysierte Reaktion liefert nach chromatographischer Aufarbeitung in guter Ausbeute das gewünschte Acetoacetat 52. Die weitere Transformation des Acetoacetats mittels Retro-Claisen-Reaktion und nachfolgendem Diazogruppentransfer liefert nach Chromatographie in ebenfalls akzeptabler Ausbeute den Diazokoester $\mathbf{5 3}$.

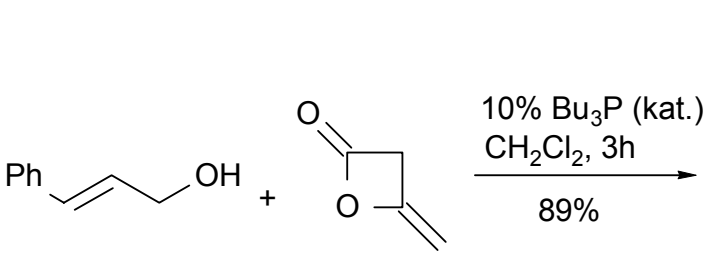

51

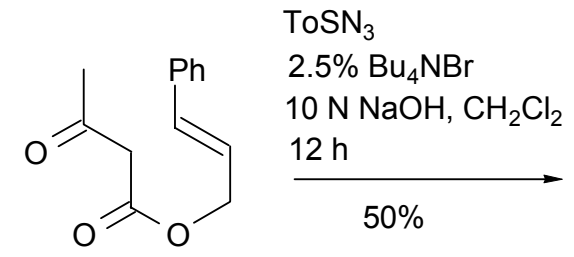

52

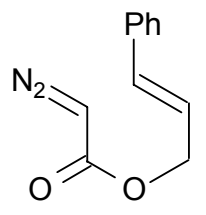

53

Schema 12. Synthese des Cinnamyldiazoacetats 53.

Für die Cyclopropanierung von Alkenen mit Diazoacetaten sind in der Literatur $^{[43]}$ viele Übergangsmetallkatalysatoren beschrieben. Gute Erfolge bei derartigen Carbenadditionen wurden durch homogene Katalysatoren erzielt. Im vorliegenden Fall wurden die Katalysator/Lösungsmittelgemische Rhodiumacetat $/ \mathrm{CH}_{2} \mathrm{Cl}_{2}$ und Kupferacetylacetonat/Kupfersulfat/Benzol getestet. Der Einsatz von Kupferacetylacetonat in siedendem Benzol gab im vorliegenden Fall die besten Ergebnisse. Mit Rhodiumacetat als Katalysator entstand ausschließlich das Dimer 53a aus dem Cinamyldiazoacetat 53. 


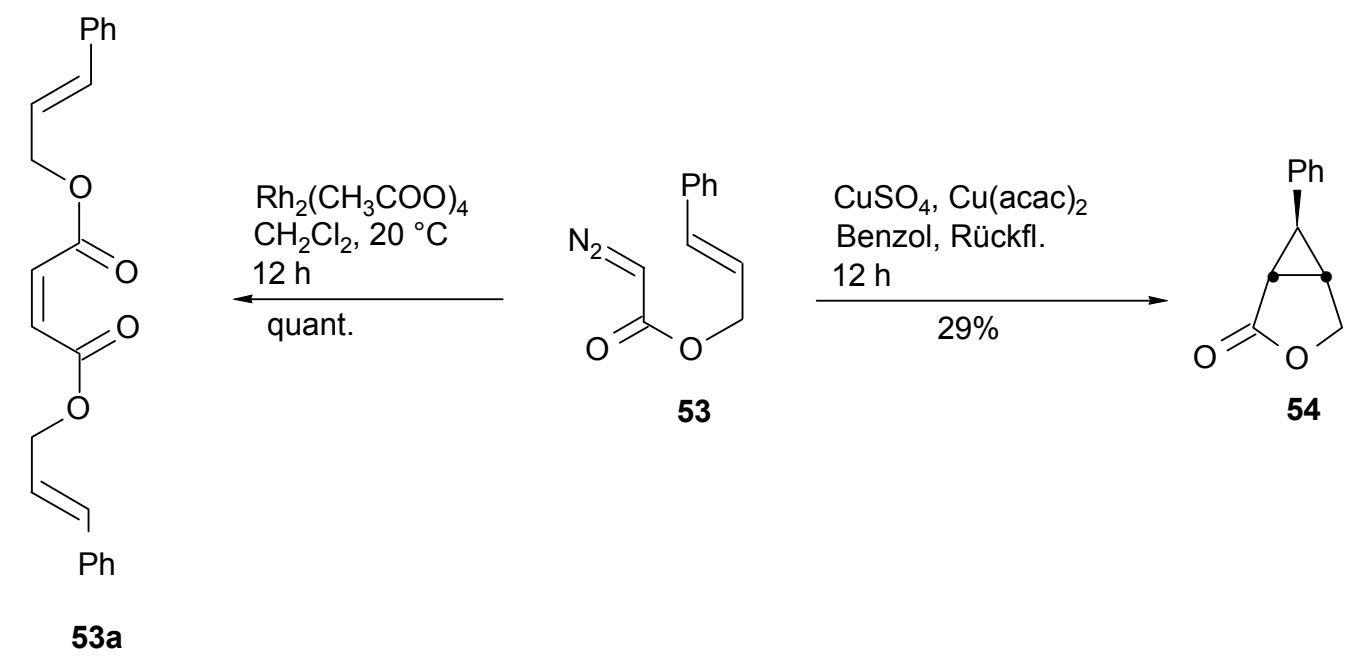

Schema 13. Intramolekulare Cyclopropanierung des Diazoessigsäureallylesters 53.

Bei der Substanzklasse der Diester 35 hatte sich bei Isomerisierungsversuchen gezeigt, dass unter verschiedenen basischen Bedingungen keine Isomerisierung erzielt werden kann. Die Konfiguration des Lactons 54 ist deshalb besonders wichtig, weil im späteren Reaktionsverlauf auch nach Ringöffnung mit Lithiumaluminiumhydrid eine Isomerisierung nicht erwartet wird.

Die unzweifelhaft beste Methode zur Konfigurationszuordnung ist die Kristallstrukturanalyse per Röntgenbeugung. Da es nicht gelang, von $\mathbf{5 4}$ geeignete Kristalle zu züchten, wurde die relative Konfiguration mittels NMR-Spektroskopie ermittelt.

Bei verschiedenen Serien von aufgenommenen NMR-Spektren fällt auf, dass die drei Signale der Cyclopropylprotonen in Deuterobenzol einwandfrei aufgelöst werden, während in Deuterochloroform nur zwei Signalgruppen für die Cyclopropaneinheit auszumachen sind.

Das ${ }^{1} \mathrm{H}-\mathrm{NMR}-$ Spektrum von 54 in Benzol zeigt kleine Kopplungskonstanten von ${ }^{3} \mathrm{~J}=3 \mathrm{~Hz}$ zwischen den Protonen $\mathrm{H}^{1}, \mathrm{H}^{2}$ und $\mathrm{H}^{3}$, wie sie für das exo-Diastereomere 54a erwartet wird. Cis-ständige Protonen am Cyclopropan weisen im allgemeinen eine größere ${ }^{3} J$ Kopplungskonstante (ca. $6 \mathrm{~Hz}$ ) auf. Man würde deshalb für 54a ein Spektrum erwarten, das eine große Kopplungskonstante zwischen $\mathrm{H}^{1}$ und $\mathrm{H}^{2}$ und zwei kleinere der Protonen $\mathrm{H}^{1}$ und $\mathrm{H}^{2}$ mit $\mathrm{H}^{3}$ aufweist. Die exo-Konfiguration lässt sich allerdings anhand des einfachen ${ }^{1}$ H-NMR-Spektrums nicht eindeutig nachweisen.

Bei Betrachtung der beiden in Erwägung gezogenen Epimere ist in Struktur 54a die räumliche Nähe der Protonen $\mathrm{H}^{1}$ und $\mathrm{H}^{2}$ mit den Arylprotonen $\mathrm{H}^{4}$ und $\mathrm{H}^{5}$ auffällig, und es liegt die Vermutung nahe, dass zwischen diesen ein Nuclear-Overhauser-Effekt auftritt, der in Struktur 
54b nicht möglich ist.

Das NOESY-Experiment ergibt tatsächlich den erwarteten NOE-Effekt. Die Konnektivität durch den Raum ist in diesem Falle eine hilfreiche Methode, da die Konstitutionsaufklärung mittels 1-dimensionalem Spektrum nicht gelugnen ist. Das Spektrum enthält intensive Kreuzpeaks für die Protonen $\mathrm{H}^{1}, \mathrm{H}^{2}$ und $\mathrm{H}^{3}$ einerseits, und den orthoständigen Protonen $\mathrm{H}^{4}$ und $\mathrm{H}^{5}$ des Phenylrings andererseits, was auf eine räumliche Nachbarschaft dieser Kerne hinweist.

Unter Annahme eines frei rotierenden Phenylrings können die Wechselwirkungen der Cyclopropylprotonen mit den Aromatenprotonen $\mathrm{H}^{4}$ und $\mathrm{H}^{5}$ leicht erklärt werden. Als Nebeneffekt dieses Experiments kann festgestellt werden, dass das mit $\mathrm{H}^{1}$ bezeichnete Proton tatsächlich das der Carbonylgruppe benachbarte Cyclopropylproton sein muß, da es im Gegensatz zu $\mathrm{H}^{2}$ und $\mathrm{H}^{3}$ keinen NOE-Effekt $\mathrm{zu}$ den Methylenprotonen $(\delta=3.5 \mathrm{ppm})$ aufweisen darf. Mit diesem Ergebnis kann die endo-Konfiguration des Lactons 54b ausgeschlossen werden. 


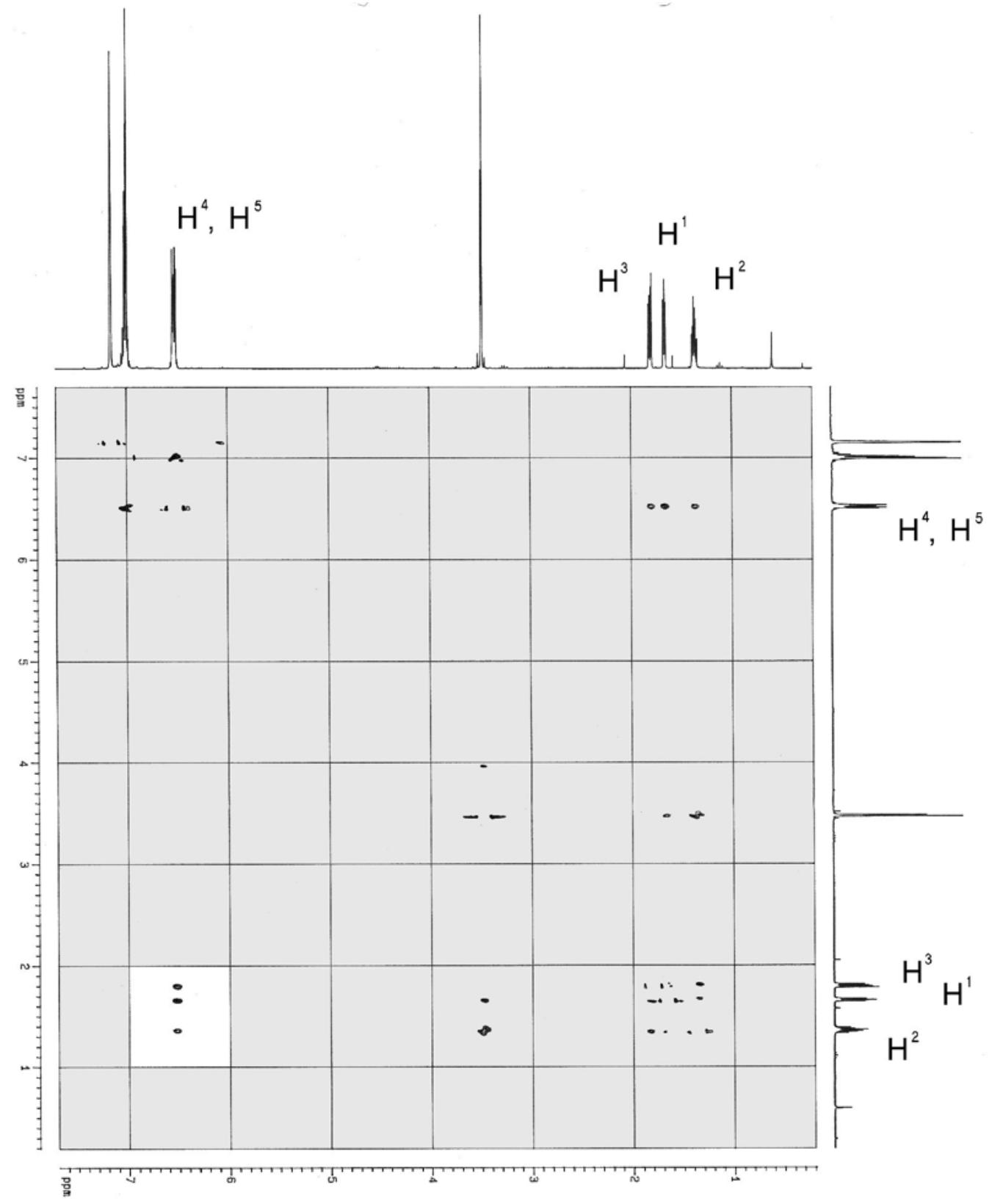

Abb. 12. Zuordnung der relativen Konfiguration des Lactons 54 mittels NOESY-Spektrum.<smiles></smiles>

$54 a$

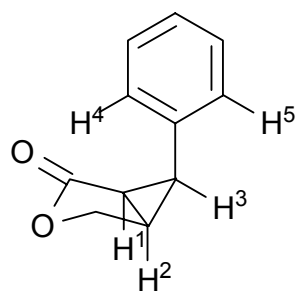

54b

Abb. 13. Exo- und endo-Diastereomere des Lactons 54. 
4.2 Synthese von 6-(4-Bromphenyl)-2-oxo-3-oxabicyclo[3.1.0]hexan

Zur Synthese von Substanzen mit möglicherweise flüssigkristallinen Eigenschaften ist die Funktionalisierung des Phenylrestes unerlässlich. Um in einem fertigen Produkt später in 4Position einen Rest der Wahl einführen zu können, wurde die Synthese eines Lactons vom Typ 54 gemäß Schema 11 wiederholt. Zur Verwirklichung dieser Synthese musste der kommerziell nicht erhältliche 4-Bromzimtalkohol durch eine Zimtsäuresynthese mit nachfolgender Reduktion der Säure hergestellt werden. Dazu wurde in der literaturbekannten Synthese $^{[44]}$ der Benzaldehyd ohne weitere Modifikation der Bedingungen durch 4Brombenzaldehyd ersetzt. Gemäß der synthetischen Idee muss das aus der Ringöffnung des Lactons resultierende Diol 55 in das entsprechende Dibromid 56 überführt werden und dann mittels Malonestersynthese den nach den Schemata 17 und 20 fehlgeschlagenen Ringschluss zu realisieren.
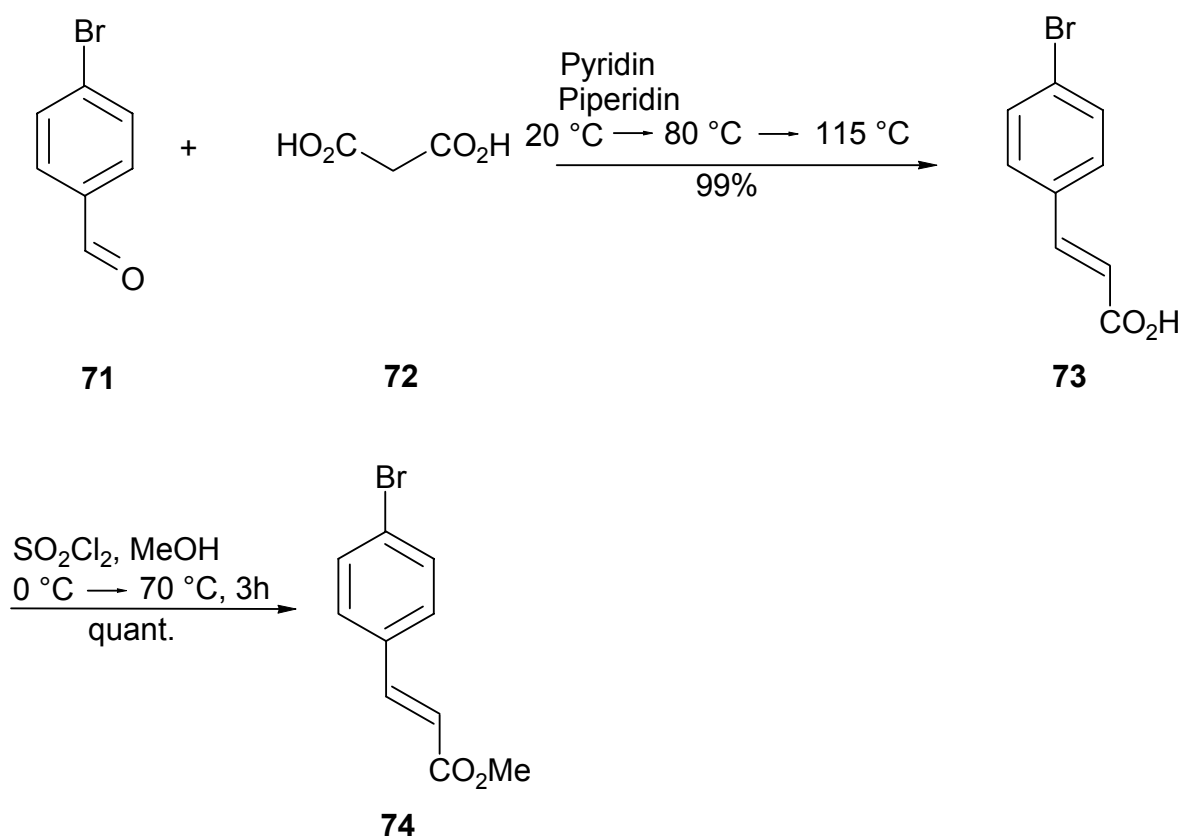

Schema 14. Zimtsäuresynthese nach J. Koo.

Dabei ergibt sich das Problem, dass die 4-Bromzimtsäure 73 zwar in sehr guter Ausbeute synthetisierbar ist, aber die nachfolgende Reduktion mit Reduktionsmitteln wie $\mathrm{LiAlH}_{4}$ oder $\mathrm{AlH}_{3}$ auch die olefinische Doppelbindung reduzieren. Die einzige im Rahmen dieser Arbeit realisierte Lösung des Problems setzt das teuere DIBAH in stöchiometrischer Menge ein. Mit dem teueren Reagenz sind die Ausbeuten an $\mathbf{7 5}$ dann aber sehr gut und das NMR-Spektrum des isolierten 4-Bromzimtalkohols $\mathbf{7 5}$ weist keine fremden Signale auf. 


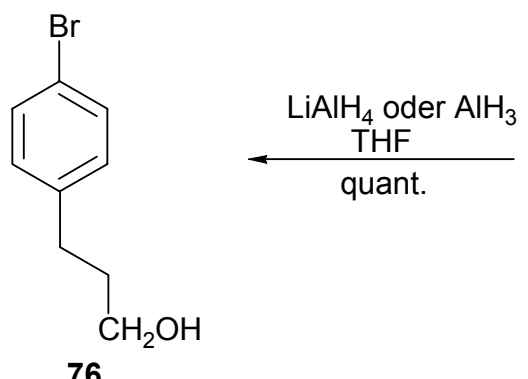

76

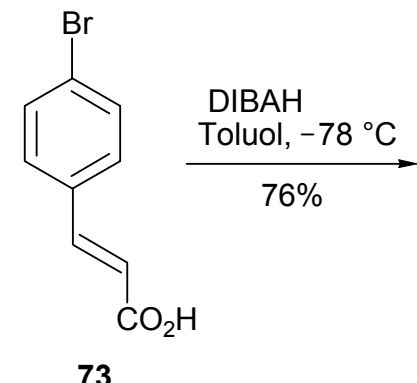

73<smiles>OC/C=C/c1ccc(Br)cc1</smiles>

75

Schema 15. Reduktion von 4-Bromzimtsäure zum 4-Bromzimtalkohol.

Die nachfolgende Synthese des Lactons nach Schema 16 verläuft mit ähnlichen Ausbeuten. Nach Doyle ${ }^{[45]}$ wurde bei dieser teureren Versuchsserie als Katalysator $\operatorname{Rh}_{2}(\mathrm{MEPY})_{4}$ verwendet, der im Fall des Phenylsubstituenten angeblich 75\% Ausbeute an isomerenreinem 54a liefern soll. Im Fall von 4-Bromcinnamyldiazoacetat wurde die Ausbeute an 78 NMRspektroskopisch jedoch mit nur 20\% abgeschätzt. Die chromatographische Reinigung des Zielproduktes an Kieselgel gelingt nicht, da das Zielprodukt sich nicht mehr vom Adsorptionsmittel entfernen lässt.

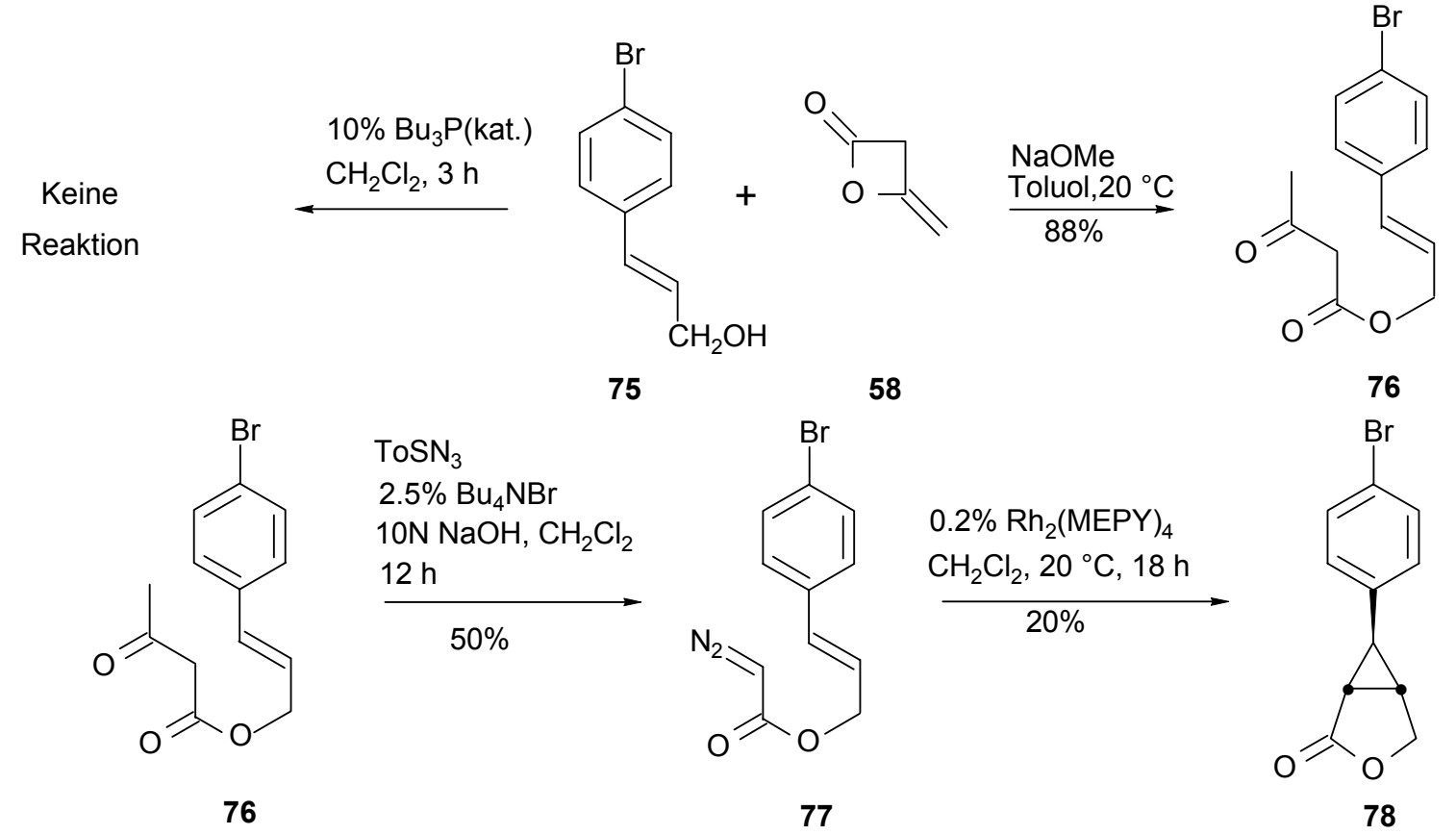

Schema 16. Synthese des 6-(4-Bromphenyl)-2-oxo-3-oxabicyclo[3.1.0]hexans 78.

Die Versuche, über die intramolekulare Variante Moleküle vom Typ 78 herzustellen, wurden wegen der beschriebenen Probleme eingestellt. Möglicherweise können die bei diesen Synthesen gewonnenen Erfahrungen aber bei der Darstellung von Substanzen vom Typ 42 44 hilfreich sein. 
4.3 Versuche zur Ringschlussreaktion von 2-Hydroxymethyl-3-phenylcyclopropylmethanol

Um aus dem isomerenreinen Lacton 54a Substanzen mit möglicherweise flüssigkristallinen Eigenschaften synthetisieren zu können, müsste der Lactonring mit Lithiumaluminiumhydrid zum Diol 60 geöffnet werden. Die beiden Hydroxyfunktionen müssen in bessere Abgangsgruppen umgewandelt werden, woraufhin ein Ringschluss über ein deprotoniertes Methylnitrat als Nucleophil erfolgen könnte.

Wie erwartet verläuft die Öffnung des Lactons sowie die Synthese des Dimesylats in nahezu quantitativer Ausbeute.

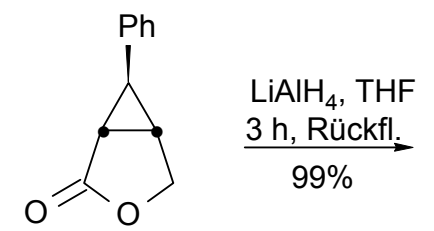

59

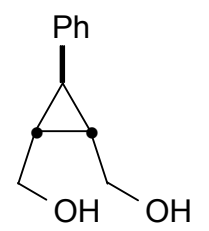

60

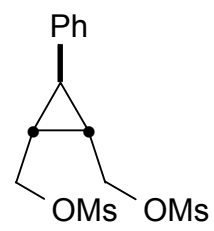

61

Schema 17. Ringöffnung des Lactons 59 und Synthese des Dimesylats 61.

Zuerst wurde versucht, das Dimesylat 61 mit Nitromethan zu kondensieren. Das dabei entstehende sekundäre Nitrat 62 ließe sich laut Literatur in ein Keton umwandeln ${ }^{[46]}$. Die dabei entstandene Ketofunktion sollte breite Möglichkeiten für die weitere Transformation eröffnen. Es ist eine Überführung des Ketons 63 mittels Wittig-Reaktion in das Alken 64 denkbar, das bei nachfolgender Hydrierung das erwünschte Zielprodukt 65 liefern könnte.

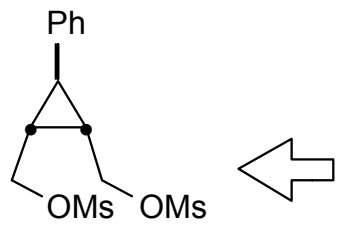

61<smiles>O=[N+]([O-])C1CC2C(C1)C2c1ccccc1</smiles>

62<smiles>O=C1CC2C3C(=O)CC3C2c2ccccc21</smiles>

63

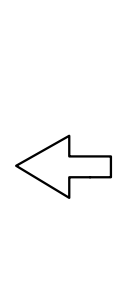

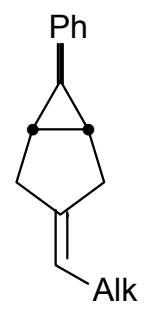

64

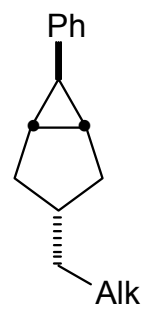

65

Schema 18. Retrosynthese von 3-Alkyl-6-phenylbicyclo[3.1.0]hexan via Wittig-Reaktion.

Eine Alternative dazu ist die Reduktion des Ketons 63 zum Alkohol 66, der nach der bekannten effektiven Methode ${ }^{[47]}$ in das entsprechende Bromid 67 überführt werden könnte. Mit diesem Bromid könnte eine übergangsmetallvermittelte C-C-Bindungsknüpfung mit einem Grignard-Reagenz zu den erwünschten Zielprodukten 69 führen. 
<smiles></smiles>

63<smiles>[CH]1CCC1</smiles>

66

6<smiles>O[C@@H]1C[C@H]2[C@@H](C1)[C@@H]2c1ccccc1</smiles><smiles>BrC1CC2C(Br)C2C1c1ccccc1</smiles>

67<smiles>[Al]C1C2C(c3ccccc3)C3C1C23</smiles>

68<smiles>[Al]C1CC2C(C1)C2c1ccccc1</smiles>

69

Schema 19. Retrosynthese von 3-Alkyl-6-phenylbicyclo[3.1.0]hexan via Reduktion des 6Phenylbicyclo[3.1.0]hexan-3-ons und übergangsmetallvermittelter C-CBindungsknüpfung

Eine weitere Alternative ist die Thorpe-Ziegler-Cyclisierung ${ }^{[48]}$ des Dinitrils $\mathbf{7 1}$ mit nachfolgender Verseifung und Decarboxylierung. Auch diese Reaktionssequenz könnte 63 als Edukt für eine Wittigreaktion zur Verfügung stellen.

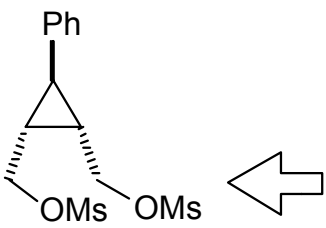

61

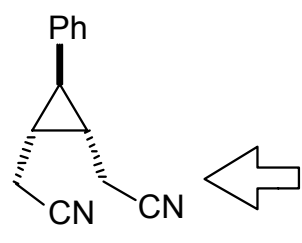

71<smiles>N#CC1=C(N)CC2C1C2c1ccccc1</smiles>

70

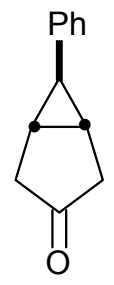

63

Schema 20. Retrosynthese von 3-Oxo-6-phenylbicyclo[3.1.0]hexan via Thorpe-Ziegler-Cyclisi erung.

Wie alle vorangegangenen Versuche zur Cyclisierung von 2-Hydroxymethyl-3phenylcyclopropylmethanol 60 ist auch diese Variante gescheitert. Einerseits konnte das Lacton 59 nicht in genügender Menge für aufwendige Versuchsreihen zur Verfügung gestellt werden, andererseits ist weder die Herstellung des Dinitrils 71 noch die Synthese des Nitrats $\mathbf{6 2}$ in ausreichender Menge und Reinheit gelungen 


\section{Synthese 4-substituierter Cyclopentene}

5.1 Retrosynthetische Überlegungen zu 4-substituierten Cyclopentenderivaten

Es ist bekannt, dass sich 4-Cyclopentenol 80 aus Cyclopentadien durch Hydroborierung mit mindestens 2 verschiedenen Methoden ${ }^{[49,50]}$ darstellen lässt. Eine ebenfalls literaturbekannte Bromierung $^{[47]}$ ergibt hieraus das 4-Bromcyclopenten 81, woraus das in guter Ausbeute synthetisierbare entsprechende Grignardreagenz 82 einen geeigneten Kupplungspartner für eine an ähnlichen Beispielen literaturbekannte ${ }^{[51]}$ metallkatalysierte Kreuzkupplung an Aromaten bietet. Die Zielprodukte $\mathbf{8 3}$ bieten die Grundlage für die Synthese der Substanzklassen $\mathbf{4 1 ,} \mathbf{4 3}$ und $\mathbf{4 5}$.

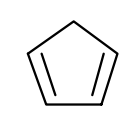

79<smiles>C1CCCC1</smiles><smiles>OC1CC=CC1</smiles>

80

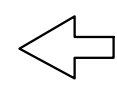<smiles>BrC1CC=CC1</smiles>

81

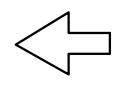<smiles>[Mg]C1CC=CC1</smiles>

82<smiles>BrC1CC=CC1</smiles>

83

Schema 21. Retrosynthese von 4-Arylcyclopentenderivaten. 


\subsection{Palladium-katalysierte Aryl-Cyclopentenyl-Kreuzkupplung}

Für die Synthese von 4-Cyclopentenol 80 stehen mehrere Wege zur Verfügung. Sie unterscheiden sich in der Methode, mit der das für die Hydroborierung erforderliche Diboran

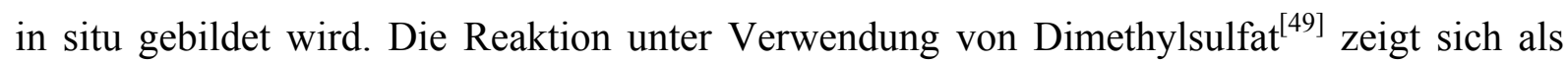
nachteilig gegenüber der Synthese unter Verwendung von Bortrifluoridetherat ${ }^{[50]}$, bei der Verunreinigungen vor der Oxidation im Vakuum entfernt werden können, und die durch die nach der Hydroborierung erfolgende Oxidation und fraktionierter Destillation gute Ausbeuten an reinem 4-Cyclopentenol 80 liefert. Die nachfolgende Synthese des 4-Bromcyclopentens 81 unter Verwendung des Triphenylphospan-Bromkomplexes ist in der Literatur ausreichend beschrieben als leistungsstarke Methode zur Bromierung von Alkoholen in Anwesenheit von olefinischen Doppelbindungen und ergibt auch bei der Bromierung von 4-Cyclopentenol 80 sehr gute Ausbeuten. Eine weitere Überführung des 4-Bromcyclopentens in das Grignardreagenz 82 ist ebenfalls in sehr guten Ausbeuten möglich.

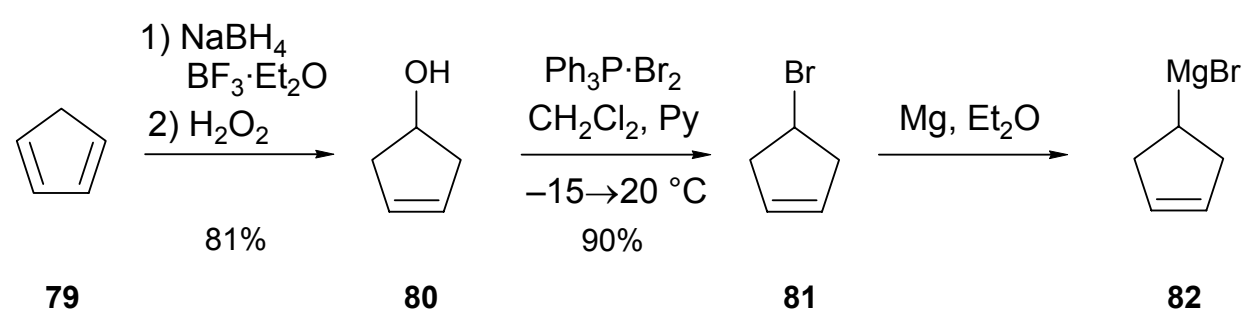

Schema 22. Synthese von 4-Cyclopentenmagnesiumbromid.

Als Kupplungspartner für die Reaktion mit Cyclopentylmagnesiumbromid stehen die bei der Synthese von Flüssigkristallen üblichen Substanzen $\mathbf{8 4 - 8 8}$ zur Verfügung. Sie unterscheiden sich hauptsächlich in ihren Substituenten. Fluorinierte Kupplungspartner sind nach dem aktuellen Trend in der Entwicklung flüssigkristalliner Substanzen sehr beliebt, die Verwendung von fluorfreien Kupplungspartnern ist wegen der guten Vergleichsmöglichkeit mit bekannten ähnlichen Molekülen aus Datenbanken ebenfalls interessant. 


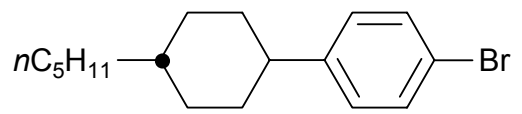

84

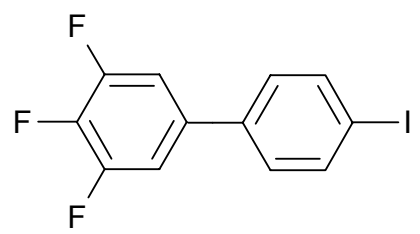

85

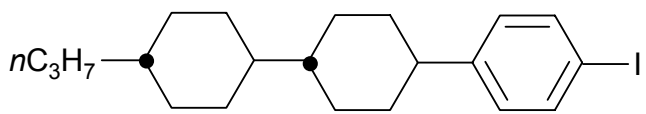

86<smiles>Fc1cc(Br)cc(F)c1F</smiles>

87<smiles>CCOc1ccc(I)c(F)c1F</smiles>

88

Abb. 14. Reaktionspartner für die Kupplung mit 4-Cyclopentenylmagnesiumbromid.

Die Kupplungsreaktionen mit den Molekülen 84 - 87 verlaufen mit der erwartet guten Ausbeute, die Synthese des Zielmoleküls 93 durch Kupplung mit 88 misslingt aus nicht näher erklärbaren Gründen. Mit Ausnahme der Darstellung von 4-(4-Ethoxy-2,3difluorphenyl)cyclopenten mit 88, bei der 4-(4-Ethoxy-2,3-difluorphenyl)jodid unverändert zurückgewonnen wurde, stellt die palladiumkatalysierte Kreuzkupplung von Cyclopentylmagnesiumbromid mit Arylhalogeniden 84 - 87 eine leistungsfähige Methode zur Synthese der Edukte für die nachfolgende Addition von Carbenen oder Carbenoiden dar. Eine Übersicht über die Ausbeuten der jeweiligen Reaktionen findet sich in Tabelle 2.

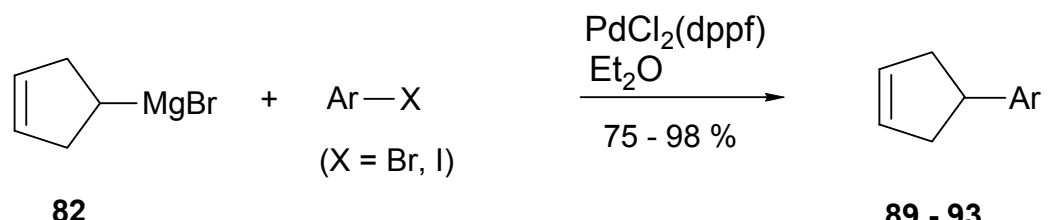

Schema 23. Pd-katalysiere Kreuzkupplung von 4-Cyclopentenylmagnesiumbromid mit verschiedenen Arylhalogeniden. Details siehe Tabelle 2. 
Tabelle 2: Synthesen von 4-Arylcyclopentenen

84 Produkt $\quad$ Ausbeute (\%)




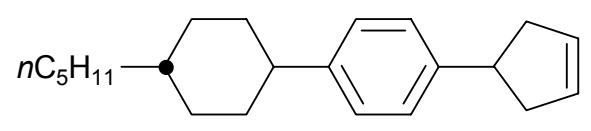

89

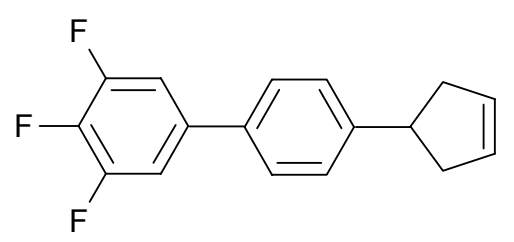

90<smiles>Fc1cc(C2CC=CC2)cc(F)c1F</smiles>

92

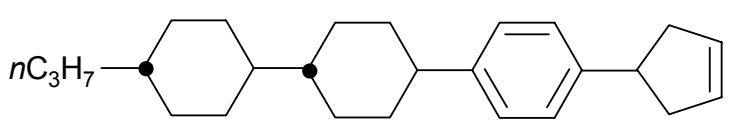

91<smiles>CCOc1ccc(C2CC=CC2)c(F)c1F</smiles>

93

Abb. 15. Zielmoleküle der Pd-katalysierten Kreuzkupplung.

5.2 Cuprat-katalysierte Alkyl-Cyclopentenyl-Kreuzkupplung

Aus den in den voranstehenden Kapiteln vorgestellten Vorläufern 4-Cyclopentenol 80 (Schema 22) und 4-Cyclopentencarbonsäure 31 (Schema 6) lassen sich das4-Bromcyclopent1-en (81) und das 4-(Brommethyl)cyclopent-1-en (95) und daraus die Grignard-Reagenzien 82 und 96, als Substrate für Cuprat-katalysierte Kreuzkupplungen nach Nicolau ${ }^{[52]}$ herstellen.<smiles>O=C(O)C1CC=CC1</smiles>

31

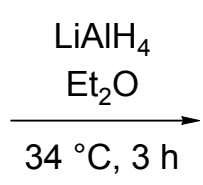

$91 \%$<smiles>OCC1CC=CC1</smiles>

94

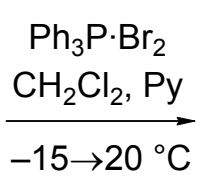

$85 \%$<smiles>BrCC1CC=CC1</smiles>

95<smiles>CCOCC</smiles><smiles>Br[Mg]CC1CC=CC1</smiles>

96

Schema 24. Darstellung von Cyclopent-3-en-1-ylmethylenmagnesiumbromid (96). 
Durch diese Kreuzkupplung können primäre Grignard-Reagenzien mit primären Halogeniden verknüpft werden. Die Kupplung zweier sekundärer Reaktionspartner gelingt nur in einigen Ausnahmefällen. Mit der Cuprat-katalysierten Methode können somit Substanzen des Typs 37 hergestellt werden. Das Cyclopentenderivat 94 kann wahlweise in das Halogenid und nachfolgend in das Grignard-Reagenz überführt werden. Die Kupplungspartner können entweder jeweils in einstufigen Synthesen synthetisiert werden, wenn sie nicht käuflich sind. Bei dem Versuch 98, mit dem Grignard-Reagenz 96 zu kuppeln, entstand als Hauptprodukt das Diarylmethanderivat 99, das Kupplungsprodukt 100 konnte nur in 25\% Ausbeute isoliert werden.

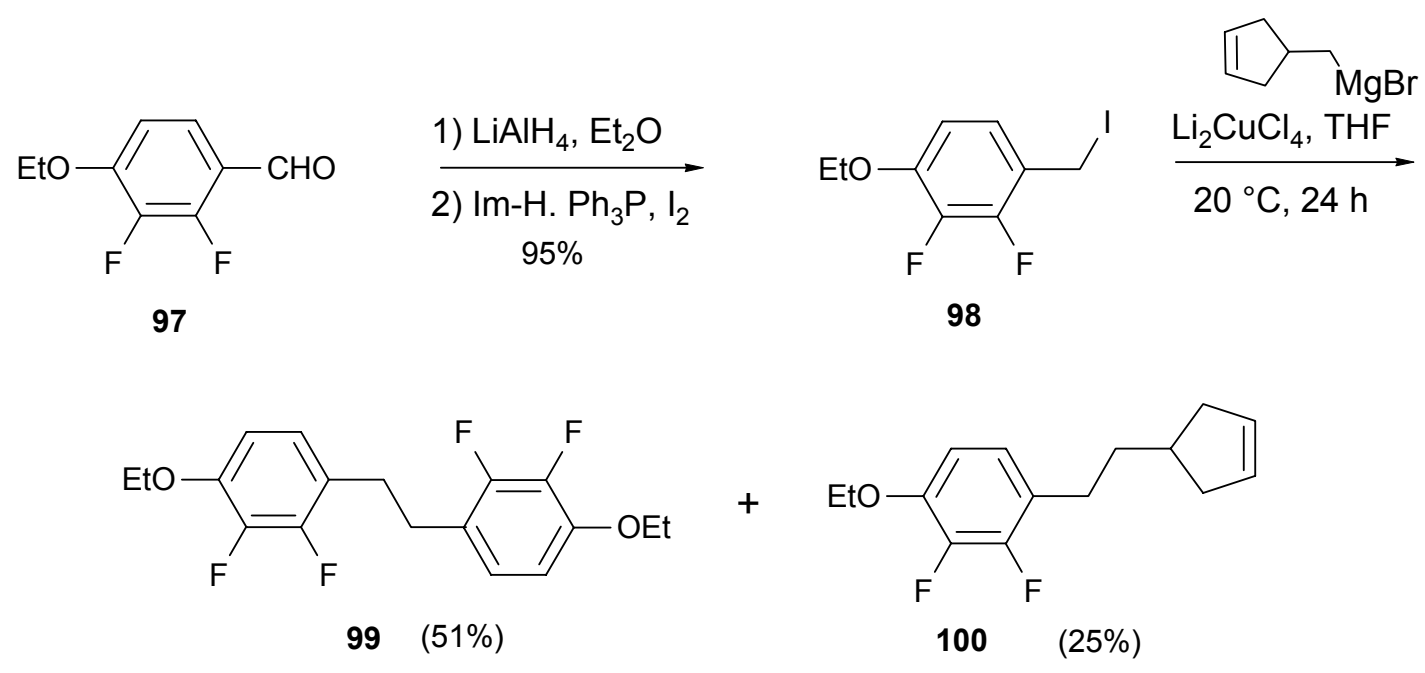

Schema 25. Synthese von 1-Ethoxy-,2,3-difluor-4-[2-(cyclopent-3-en-1-yl)ethyl]benzol 100. 
Die Kupplung des 4-(Iodmethyl)cyclopentens (101) zweier mit Ethylmagnesiumbromid verlief in 54\% Ausbeute (Schema 26). Erstaunlicherweise lieferte die Kupplung mit dem sekundären Cyclopentenyl-4-magnesiumbromid (82) vergleichbare Ausbeuten. Wegen des einfacheren Zugangs $\mathrm{zu}$ 4-Bromcyclopenten (81) wurden deshalb verschiedene 4-Alkylcyclopentene durch Kupplung von 4-Alkyliodiden mit dem sekundären GrignardReagenz dargestellt (Schema 27). Damit standen mehrere 4-Alkylcyclopentenderivate als Edukte für die spätere Addition von Carbenen oder Carbenoiden zur Verfügung.<smiles>OCC1CC=CC1</smiles>

94

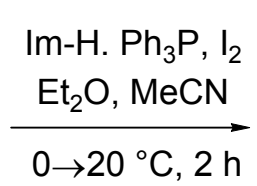

$84 \%$

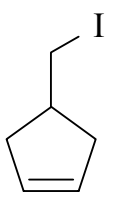

101

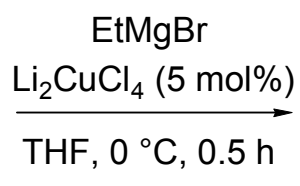

$54 \%$

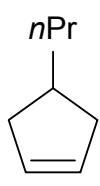

102

Schema 26. Synthese von 4-Alkylcyclopentenen aus Cyclopenten-4-ethanol.

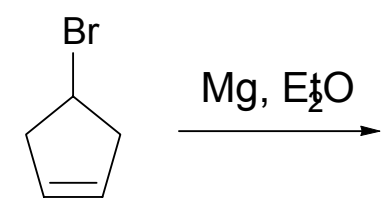

81<smiles>Br[Mg]C1CC=CC1</smiles>

82

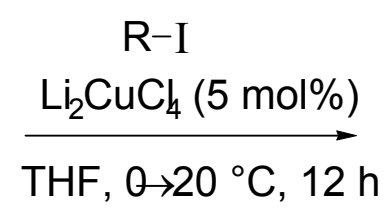<smiles>[R]C1CC=CC1</smiles>

Schema 27. Synthese von 4-Alkylcyclopentenen aus 4-Bromcyclopenten.

Tabelle 3. Ausbeuten der Synthese von 4-Alkylcyclopentenen aus 4-Bromcyclopenten.

\begin{tabular}{ccc}
\hline $\mathrm{R}$ & Produkt & Ausbeute (\%) \\
\hline$n \operatorname{Pr}$ & $\mathbf{1 0 2}$ & 30 \\
$n$ Pent & $\mathbf{1 0 3}$ & 53 \\
$n$ Hept & $\mathbf{1 0 4}$ & 56 \\
\hline
\end{tabular}




\subsection{Synthese von 4-Cyclohexylcyclopentenen}

\subsubsection{Vorüberlegungen und synthetische Ansätze}

Zur Darstellung der Substanzklassen 33 und 35 mußte eine Möglichkeit zur Kupplung zweier sekundärer Kohlenstoffatome gefunden werden. Es ist aus Erfahrung bekannt, dass sogar die Kupplung von Cylohexanon mit Methyllithium Organometallverbindungen schlechte Ausbeuten liefert, da die Organometallkomponente als starke Base mit sterisch anspruchsvollen Ketonen zur Enolatbildung führt. Nachfolgende Hydrolyse führt zur Entstehung eines Alkans aus dem Grignardreagenz sowie zur Rückbildung des Ketons. So führt die Reaktion von 4-nPentylcyclohexanon 106 mit dem entsprechenden Lithiumorganyl 82a nur zur Rückgewinnung der gesamten eingesetzten Menge an Keton 106. Das dabei entstandene Cyclopenten (105) wurde offenbar aufgrund des niedrigen Siedepunkts mit dem Lösungsmittel entfernt.

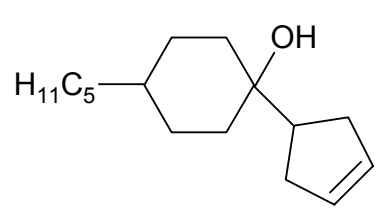

107

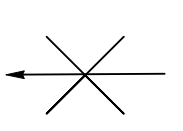<smiles>ClC1CC=CC1</smiles>

$82 a$

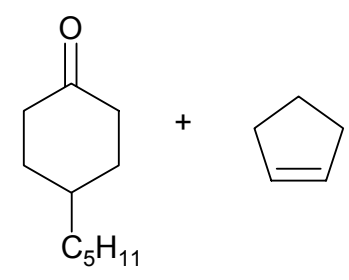

106
105

Schema 28. Reaktion von Cyclohexanon mit Cyclopentenylmagnesiumbromid.

Ein weiterer vielversprechender Ansatz ist die Ringschlussreaktion über den Cyclopent-3encarbonsäuremethylester 109. Ähnliche Reaktionen mit $\alpha, \omega$-Bis-Grignard-Reagenzien sind literaturbekannt ${ }^{[53]}$ und liefern in einigen Fällen gute Ausbeuten. So kann Pentan-1,5-diyl-bismagnesiumbromid in Ausbeuten von 56\% mit Buttersäureethylester zum erwünschten Ringschlussprodukt ${ }^{[53]}$ abreagieren. In der hier zu versuchenden Variante ist die Substitution in 3-Position des Grignard-Reagenzes von entscheidender Bedeutung für die späteren physikalischen Eigenschaften des Zielproduktes. Grignard-Reagenzien bieten sich auch hier wegen ihrer angepassten Reaktivität und ihrer leichten Darstellbarkeit an. 


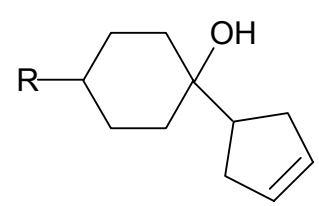

110

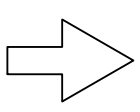

Schema 29. Retrosynthetische Überlegungen zu 1-(4-Cyclopentenyl)-4-alkyl-1-<smiles></smiles>

108

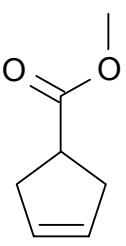

109 cyclohexanolen.

Der Zugang zu Metallorganylen vom Typ 108 erweist sich als unerwartet schwierig. Nicht die Möglichkeit, ein 3-Alkyl-1,5-dibrompentan in die jeweilige Grignardverbindung zu überführen bereitet Probleme, sondern die Einführung eines innerhalb eines kurzen Synthesewegs variablen Substituenten in der 3-Position erfordert gründliche synthetische Überlegungen. Gewöhnlich werden Dibromide vom Typ $\mathbf{7 0}$ durch Ringöffnung 4-substituierter Tetrahydropyranderivate hergestellt ${ }^{[54]}$. Dieser Syntheseweg ist aber ungünstig, weil das käuflich erhältliche Edukt teuer ist, und weil sich der Alkylsubstituent später nicht mehr variieren lässt. Aufgrund der beschriebenen Nachteile wurde beschlossen, eine günstigere Synthese zur Herstellung der 3-Alkyl-pentan-1,5-diyl-bismagnesiumbromide 108 zu suchen. Es sind einige in verschiedenen Quellen ${ }^{[55][56][57][58]}$ beschriebene Synthesen ausgehend von dem billigen $\delta$-Valerolacton 111 mit verschiedenen Erfolgen erprobt worden. 


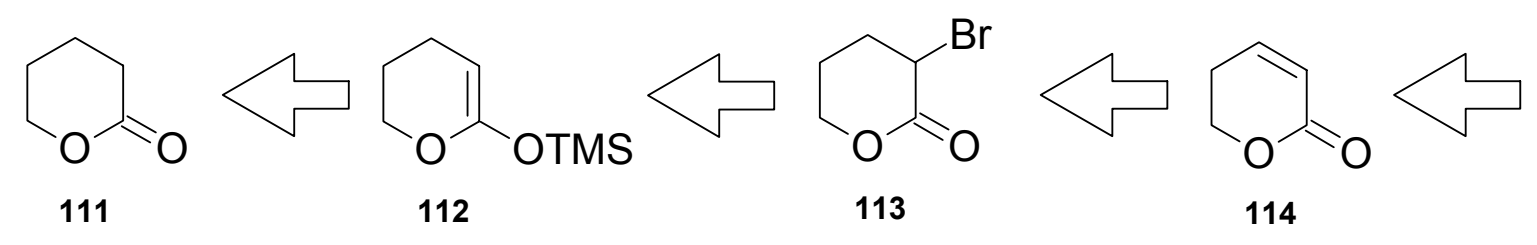

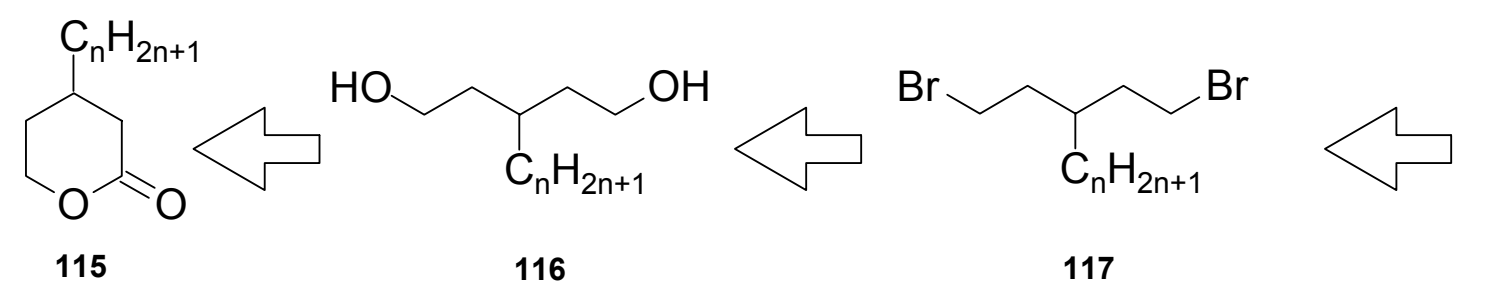

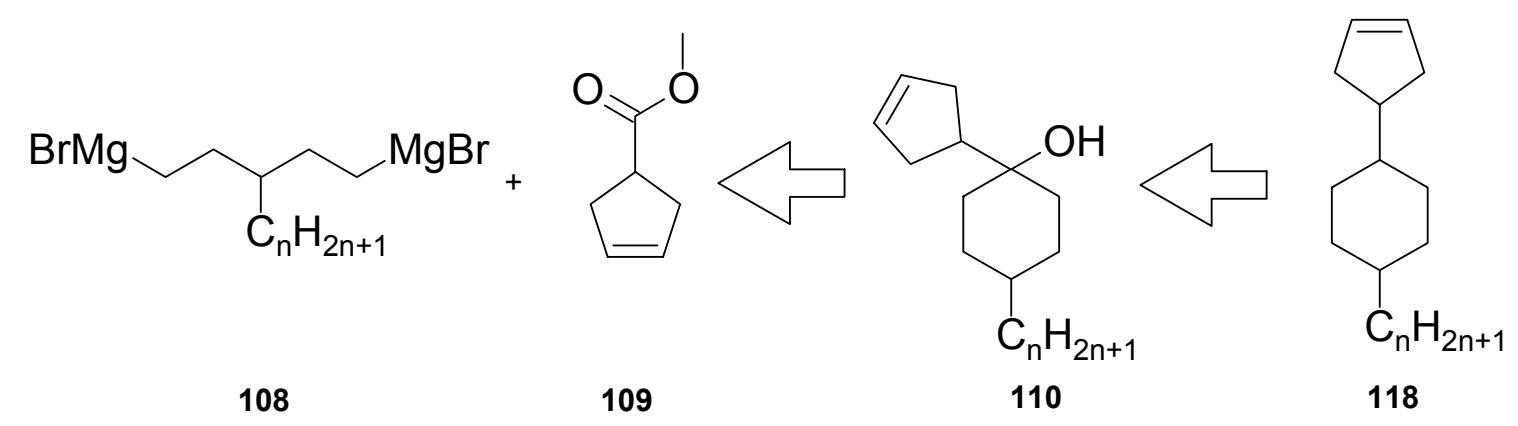

Schema 30. Retrosynthetische Überlegungen zu 1-(3-Cyclopentenyl)-4-alkylcyclohexanDerivaten.

5.3.2 Versuche zur Synthese von Cyclohexylcyclopentenylderivaten aus $\delta$-Valerolacton

Literaturgemäß $^{[59]}$ lässt sich die Synthese des 5,6-Dihydropyran-2-yltrimethylsilylethers 112 in guter Ausbeute problemlos nachvollziehen. Nach einer ebenfalls literaturbekannten Vorschrift $^{[55]}$ sollte sich dieser in guter Ausbeute in das 3-Bromtetrahydro-2-pyranon 113 überführen lassen. Danach ${ }^{[60]}$ sollte das Reaktionsprodukt ein braunes Öl mit einigen Verunreinigungen sein, die bei weiteren synthetischen Schritten angeblich nicht stören sollten.

In dem nachvollzogenen Versuch fiel bei allen Experimenten ein tiefbraunes Öl mit gemäß ${ }^{1}$ H-NMR-Spektrum mindestens $20 \%$ nicht abtrennbaren Verunreinigungen an. Durch Destillation konnte zwar ein gelbliches Öl erhalten werden, dessen NMR-Spektren sich aber von denen des braunen Öls nicht unterschieden. Weiterführende synthetische Versuche zur Dehydrobromierung von 113 mit verschiedenen Basen und Lösungsmitteln schlugen fehl. 


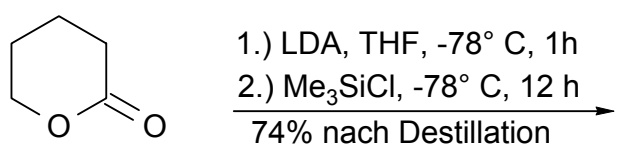

111<smiles>COC1=CCCCO1</smiles>

112

Schema 31. Synthese des 5,6-Dihydropyran-2-yltrimethylsilylethers 112.<smiles>O=C1OCCCC1Br</smiles>

113

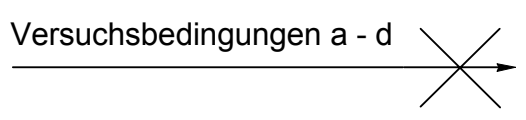

(1)

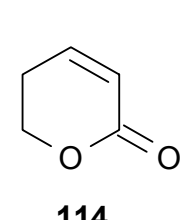

114 a) $\mathrm{KO}^{\mathrm{t} B u}$, THF, RT

b) Pyridin, Benzol, reflux

c) $\mathrm{Et}_{2} \mathrm{O}, \mathrm{NEt}_{3}$, reflux

d) $\mathrm{K}_{2} \mathrm{CO}_{3}, 120^{\circ} \mathrm{C}, 3 \mathrm{~h}$, $10^{-2}$ Torr

Schema 32. Dehydrobromierung von 3-Bromtetrahydro-2H-2-pyranon 113.

Eine erweiterte Beilstein-Crossfire Recherche ergab, dass es möglich ist, Palladiumkatalysiert mit Hilfe von Allylmethylcarbonat $\mathbf{1 2 0}$ den 5,6-Dihydropyran-2yltrimethylsilylether 112 in 70\% Ausbeute $^{[61]}$ in das 5,6-Dihydro-2H-2-pyranon $114 \mathrm{zu}$ überführen. Dieses Verfahren hat den Nachteil, dass das stöchiometrisch eingesetzte Carbonat 120 zwar käuflich, aber sehr teuer ist. Deshalb wurde das Allylmethylcarbonat nach einem deutschen Patent ${ }^{[62]}$ in einer haloformähnlichen Reaktion aus Trichloressigsäure 119 und Allylalkohol 118 synthetisiert.

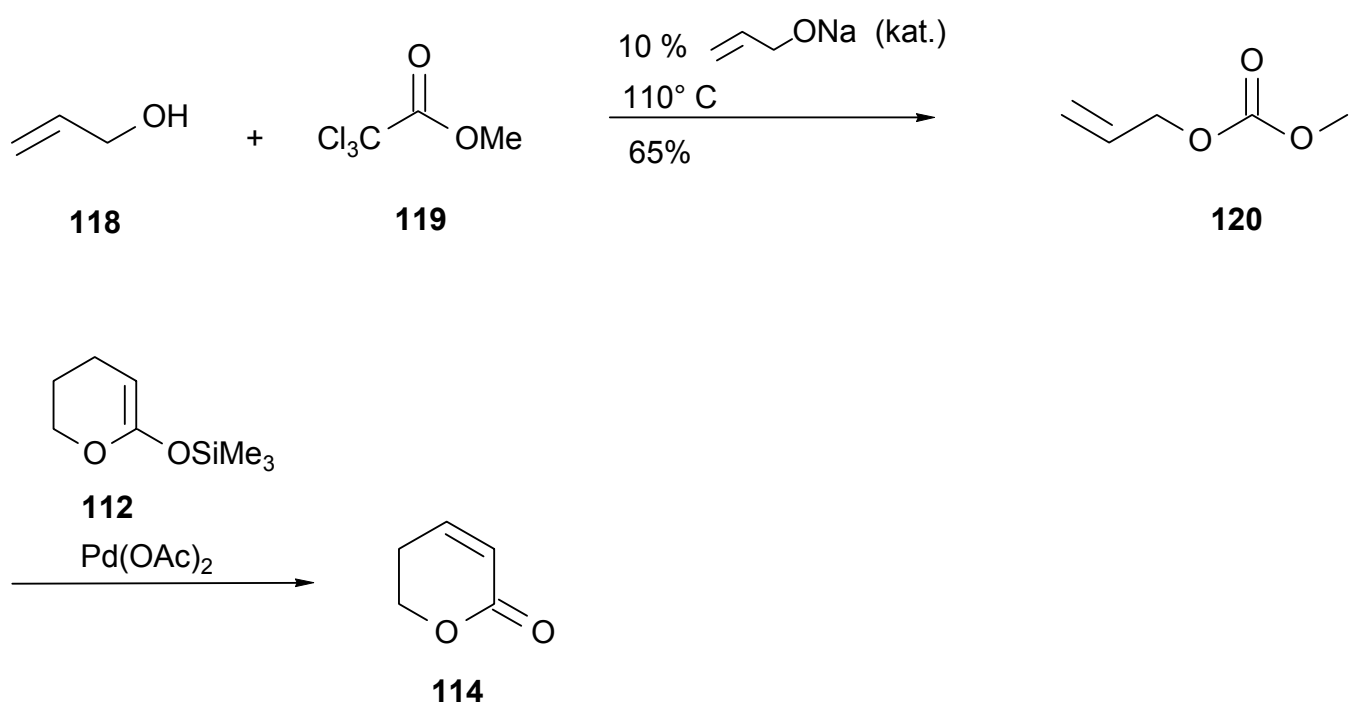

Schema 34. Synthese von 5,6-Dihydro-2H-2-pyranon 114.

Die Lactonstrategie nach Schema 30 wurde daher zugunsten der besseren und schnelleren Idee einer Cyanacetamidkondensation mit nachfolgender Michael-Addition aufgegeben. 


\subsubsection{Cyanacetamidkondensation mit nachfolgender Michael-Addition}

Die Lactonstrategie erwies sich als schwierig und langwierig. Sie ist kaum geeignet, einige hundert Gramm Edukte darzustellen. Mit der literaturbekannten ${ }^{[63]}$ Cyanacetamidkondensation mit nachfolgender Michael-Addition eines aliphatischen Aldehyds bietet sich eine Möglichkeit, diese Aufgabe zu lösen. Das entstehende 2,4-Dicyano-3-alkylglutarsäurediamid 122 lässt sich literaturgemäß ${ }^{[63]}$ durch Erhitzen in die 3-Alkylpentan-1,5disäure 121 überführen. Die Reduktion von Säuren zu Alkoholen verläuft erfahrungsgemäß problemlos. Die nachfolgende milde Bromierung mit dem Triphenylphosphan-Bromkomplex liefert auch bei weitaus komplizierteren Molekülen mit mehreren Funktionalitäten gute Ergebnisse.<smiles>[R]C(CCBr)CCBr</smiles>

$70 \mathrm{a}$

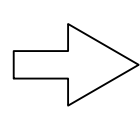

$\mathrm{HO}$<smiles>[R]C(CCC)CCO</smiles>

$71 \mathrm{a}$

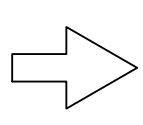<smiles>[R]C(CC(=O)O)CC(=O)O</smiles>

121<smiles>[R]C(C(C#N)C(N)=O)C(C#N)C(N)=O</smiles><smiles>C1CCCCC1</smiles><smiles></smiles>
122

Schema 35. Retrosynthetische Überlegungen zu 3-Alkyl-1,5-Dibrompentan.

5.3.4 Synthese eines 3-Alkyl-1,5-diylbismagnesiumbromids mit nachfolgender Additionsreaktion

Die literaturbekannte ${ }^{[63]}$ basenkatalysierte Cyanacetamidkondensation mit nachfolgender Michael-Addition von Butanal kann mit mehreren hundert Gramm Ausgangsmaterial in wässrigem Medium als "Eintopfreaktion" durchgeführt werden. Das entstehende 2,4Dicyano-3-propyl-glutarsäurediamid 122 ist nicht wasserlöslich und fällt vollständig aus. Das farblose Reaktionsprodukt kann durch Umkristallisieren aus Salzsäure weiter aufgereinigt werden, was sich im Falle dieser synthetischen Verwendung als nicht notwendig erwies, da 
die NMR-Spektren keine Signale von Verunreinigungen enthielten. Die nachfolgende Umsetzung in $6 \mathrm{~N}$ Salzsäure verläuft ebenso schnell und problemlos, wie die Synthese des 2,4-Dicyano-3-alkylpentandiamids 122 und liefert in guter Ausbeute die 3-n-Propyl-1,5pentandisäure 121, deren weitere Reinigung für diese synthetische Anwendung nicht erforderlich ist.

Die Reduktion der 3-n-Propylglutarsäure 121 zu 3-n-Propyl-1,5-pentandiol 71 mit Lithiumaluminiumhydrid verläuft wie erwartet mit guter Ausbeute. Das einzige Problem dieser Reaktion ergab sich bei der Scalierung zum Molmasstab. Es erweist sich als äußerst schwierig, die Wärme-, Schaum- und Wasserstoffentwicklung bei der Hydrolyse des überschüssigen Lithiumaluminiumhydrids zu kontrollieren. Dieses Problem lies sich aber mit ausreichend Erfahrung und einigem apparativen Aufwand bewerkstelligen. Auch die Transformation des Diols 71 zum Dibromid 70 kann mit Erfolg durchgeführt werden. Das synthetisierte 1,5-Dibrom-3-n-propylpentan 70 wird bei längerem Stehen bei Raumtemperatur etwas gelblich, die Reaktivität und Reinheit gemäß NMR-Spektren ändern sich dabei nicht.

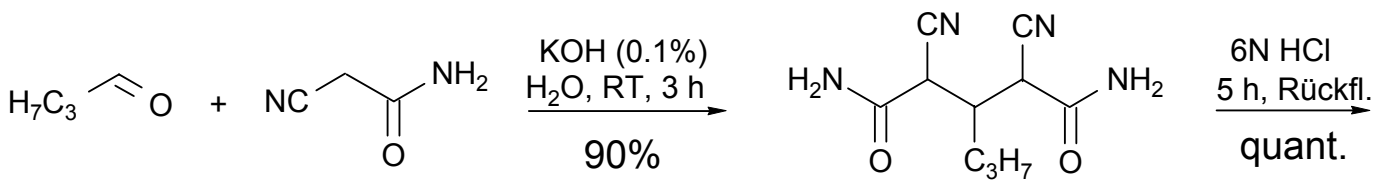

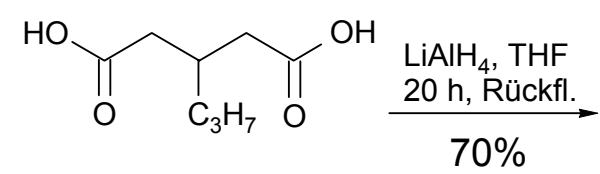

121<smiles>BrCCCC(CCBr)C(Br)Br</smiles>

70<smiles>CCC(CCO)CCO</smiles>

71

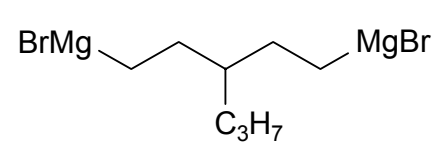

67
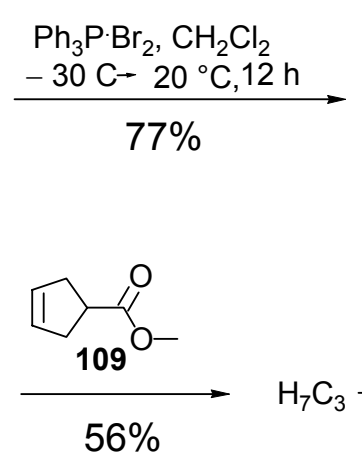<smiles>CCCCCCCCCCCCC1(O)CCC(C2CC=CC2)CC1</smiles>

123

Schema 36. Synthese von 1-Cyclopent-3-enyl-4-n-propylcyclohexanol 123.

Bei der Reaktion des 3-n-Propyl-1,5-dibrompentans 70 mit Magnesium werden der Literatur $^{[53]}$ und eigenen Versuchen zufolge je Bromatom nur 0.8 Äquivalente Magnesium eingesetzt. Der nachfolgende Ringschluss mit Cyclopenten-4-carbonsäuremethylester ergibt nach chromatographischer Reinigung in 56proz. Ausbeute das gewünschte 1-Cyclopent-3enyl-4-n-propylcyclohexanol 123. 
Mit dem 1-Cyclopent-3-enyl-4- $n$-propylcyclohexanol (123) steht nun ein Synthesebaustein zur Verfügung, an dessen olefinische Doppelbindung ein Carben oder ein Carbenoid addiert werden kann. Die Alkoholfunktionalität kann dazu entweder in ein für flüssigkristalline Substanzen günstiges Fluorid überführt ${ }^{[64,65]}$ oder später ganz entfernt ${ }^{[66]}$ werden. Unter bestimmten Umständen ${ }^{[67]}$ ist auch eine Carbenoidaddition in Anwesenheit von Hydroxyfunktionalitäten möglich, deren Realisierbarkeit wird aber hier nicht erwartet. Bei dem Versuch ein Carbenoid an die Doppelbindung zu addieren, sollte die Hydroxyfunktion wegen der hohen Acidität des Hydroxyprotons und dessen Neigung zur Insertion von Carbenoiden zuvor geschützt werden. Nach erfolgreicher Addition des Carbenoids könnte der Alkohol radikalisch nach Barton und McCombie ${ }^{[66]}$ defunktionalisiert werden. Eine solche Synthese wäre der Reduktion mit Triethylsilan ${ }^{[68]}$ über eine kationische Zwischenstufe vorzuziehen, da letztere leicht eine Ringerweiterung durch Wagner-Meerwein-Umlagerung ${ }^{\text {[69] }}$ unter Bildung einer Spiroverbindung eingeht. 


\section{Synthese und Addition von Carbenoiden an 4-substituierte Cyclopentenderivate}

Im Rahmen der Synthese flüssigkristalliner Substanzen mit zentraler Bicyclo[3.1.0]-hexanEinheit ist die Darstellung von Carbenen oder Carbenoiden und ihre Addition an 4substituierte Cyclopentenderivate von entscheidender Bedeutung. Es gibt eine fast unerschöpfliche Menge an Literatur über die Addition $^{[70]}$ von Ethyldiazoacetat an Doppelbindungen. Diese Additionsreaktion wurde auch schon zur Synthese der Diester 35a und 30b genutzt. Um Moleküle vom Typ 42-45 darstellen zu können, ist es nötig, weitere funktionalisierte Carbenoide zu synthetisieren. Gemäß dem synthetischen Ziel zur Zeit aktueller neuer flüssigkristalliner Substanzen sollten die Carben- oder Carbenoidkomponenten möglichst wenig Funktionalitäten besitzen. Für Teilstrukturen 124126 müssen verschiedene Synthesewege zur Erzeugung von Carbenen oder Carbenoiden erprobt werden. Bekannte Methoden hierfür sind die Synthese von Arylchlorcarbenen, sowie die Erzeugung von Carben- bzw. Carbenoiden via Alkyl-, Cycloalkyl- oder Aryldiazomethanderivate. Desweiteren müssen Synthesewege gefunden werden, die Teilstrukturen 124-126 durch Addition dieser Carbenoide an 4-substituierte Cyclopentenderivate zu synthetisieren.

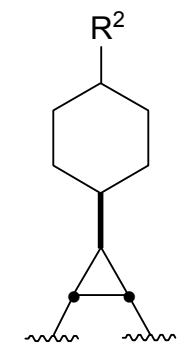

124

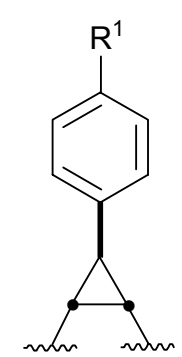

125

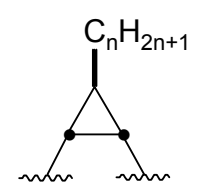

126

Abb. 16. Zielstrukturen für Carbenoidadditionen an Cyclopentenderivate. 


\subsection{Die Erzeugung von Chlorcarbenen aus Cycloalkylmethanol- oder Benlaldehydderivaten}

Es ist bekannt ${ }^{[71]}$, dass aus aromatischen Aldehyden in einer Reaktion mit Phosphorpentachlorid Aryldichlormethanderivate synthetisiert werden können. Die Behandlung dieser Dichlormethanderivate mit starken Basen in Gegenwart von Olefinen ergibt 1-Aryl-1-chlorcyclopropanderivate ${ }^{[72]}$. Es wurde auch versucht, den aus dem verfügbaren 4-(4-Ethoxy-2,3-difluorphenyl)cyclohexylmethanol (127) mittels PCC-Oxidation in quantitativer Ausbeute erhältliche Aldehyd 128 in einer analogen Reaktion einzusetzen.

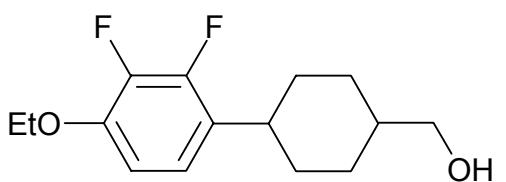

127

$$
\underset{\text { quant. }}{\stackrel{\mathrm{PCC}}{\mathrm{CH}_{2} \mathrm{Cl}_{2}, 20{ }^{\circ} \mathrm{C}}}
$$

127

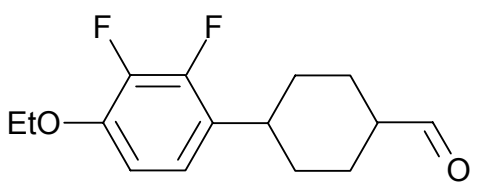

128

Schema 37. PCC-Oxidation von 4-(4-Ethoxy-2,3-difluorphenyl)cyclohexylmethanol.

Die nachfolgende Umsetzung der Aldehyde 128 und 129 mit Phosphorpentachlorid lieferte laut NMR-Spektren jeweils Produktgemische, die nur zu 35\% die zu erwartetenden Dichlormethanderivate 131 und 131 enthielten. Die chromatographische Reinigung der Dichlormethanderivate $\mathbf{1 3 0}$ und $\mathbf{1 3 1}$ schlug wegen ihrer Zersetzung bei Kontakt mit Kieselgel oder Aluminiumoxid fehl.

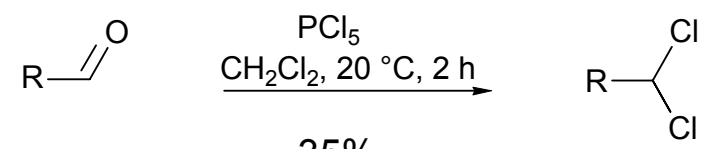
a) 128
$35 \%$
a) 130

b) 129

b) 131

a) $\mathrm{R}=$<smiles>[R][Y]([H])(C)C1CCC(c2ccc(OCC)c(F)c2F)CC1</smiles>

b) $R=$<smiles>CCOc1cccc(I)c1F</smiles>

Schema 38. Synthese der Dichlormethanderivate 130 und 131. 


\subsection{Synthese von Diazomethanderivaten aus $N$-Nitrosoharnstoffderivaten}

Für die Synthese von Diazoverbindungen sind zwei Synthesewege besonders gebräuchlich. Der erste ist die Umsetzung eines Benzylammoniumchlorids mit Harnstoff. Der gebildete $N$-Alkylharnstoff ${ }^{[73]} 135$ kann mit salpetriger Säure in das $N$-Nitrosoharnstoffderivat 136 überführt werden. Dieses wird dann für gewöhnlich durch Behandlung mit Alkalihydroxid in das jeweilige Diazomethanderivat 137 überführt $^{[74]}$.

Die zweite bekannte Synthese von Aryldiazomethanderivaten 137 verläuft über die entsprechenden Tosylhydrazone 138. Auch diese werden alkalisch pyrolysiert ${ }^{[75]}$. Bei dem milden Verfahren wird in einer Phasentransferreaktion die Diazoverbindung aus der alkalischen Glycolphase in die Hexanphase überführt. An der Färbung der Hexanphase kann erkannt werden, wann die Reaktion beendet ist.

Um etwas über die Leistungsfähigkeit der N-Aryl-N-nitrosoharnstoffvariante zu erfahren, wurde zunächst Benzylammoniumchlorid 140 mit Harnstoff in den N-Benzylharnstoff 135 überführt. Die Kondensationsreaktion liefert die erwartete quantitative Ausbeute. Der NBenzylharnstoff 135 kann entweder isoliert, oder in einer 1-Topfreaktion weiter zu dem N-Benzyl-N-Nitrosoharnstoff 136 umgesetzt werden.
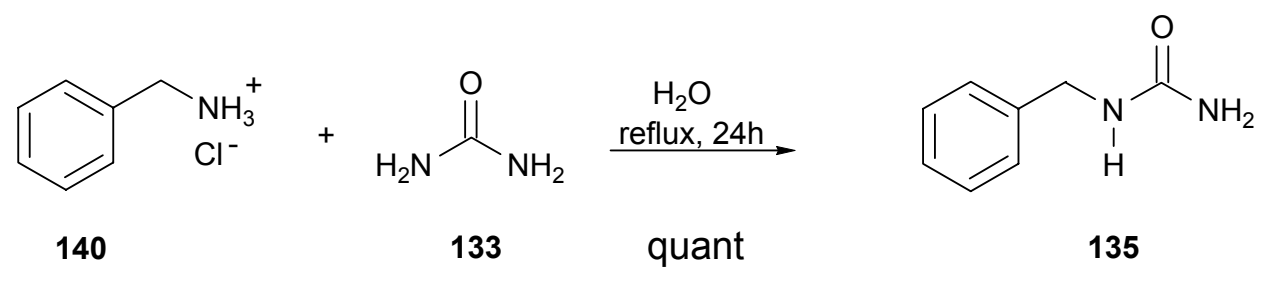

Schema 39. Darstellung von $N$-Benzylharnstoff.

Die Nitrosierung des N-Benzylharnstoff 135 ist nicht ganz unproblematisch. Die Reaktion verläuft in einer 70\%igen KOH-Lösung. Nachfolgendes Überschichten des Reaktionsgemisches mit Diethylether sollte das gewünschte Phenyldiazomethan $\mathbf{1 3 7}$ in die organische Phase überführen. Diese 2-Phasenreaktion bringt jedoch nicht den gewünschten Erfolg. Es lässt sich nur ein Bruchteil der erwarteten Menge an Phenyldiazomethan 137 in der etherischen Phase nachweisen, und das Entfernen des Lösungsmittels hat die Zersetzung der Diazoverbindung 137 zur Folge. Das NMR-Spektrum des roten Feststoffes weist eine Vielzahl von Signalen auf und die chromatographische Reinigung der Diazoverbindung 137 gelingt in diesem Fall nicht. Diese Methode ist für weitere synthetische Arbeiten ungeeignet. 


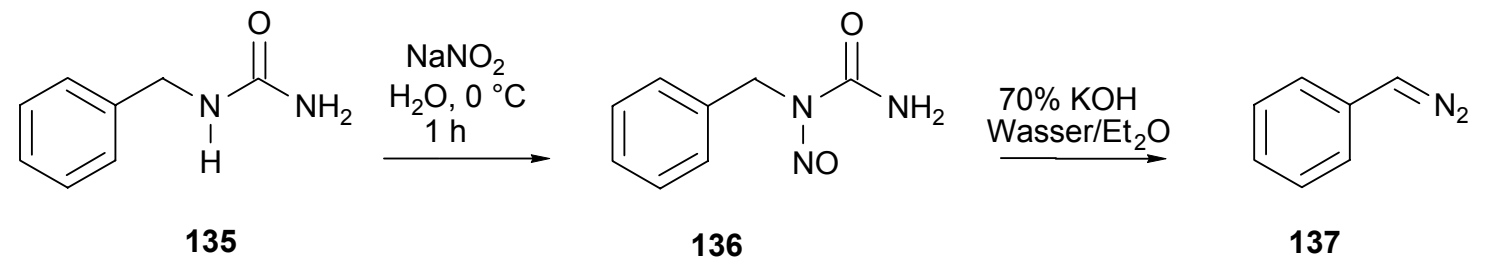

Schema 40. Synthese von Phenyldiazomethan (137) via $N$-Nitroso- $N$-phenylharnstoff. 


\subsection{Synthese von Phenyldiazomethanderivaten aus Tosylhydrazonen}

Eine weitaus vielversprechendere Methode zur Synthese der Phenyldiazomethanderivate 143 ist die alkalische Pyrolyse von Tosylhydrazonen ${ }^{[76]}$. Die benötigten Tosylhydrazone 142 sind aus den entsprechenden Aldehyden leicht und sehr schnell erhältlich. Auch bei dieser Synthesestrategie wird die unterschiedliche Löslichkeit der Tosylhydrazone $\mathbf{1 4 2}$ in Natriumglycolat/Glycollösung und Hexan ausgenutzt. Während sich die Tosylhydrazone in Hexan nicht nennenswert lösen, sind die Phenyldiazomethanderivate 143 in Hexan sehr gut, in der Glycolphase aber unlöslich.

Die entsprechende Testreaktion mit 4-Cyanobenzaldehyd 141a verlief so vielversprechend, dass die Herstellung von Aryldiazomethanderivaten mit den Aldehyden 141b und 141c erprobt wurde.

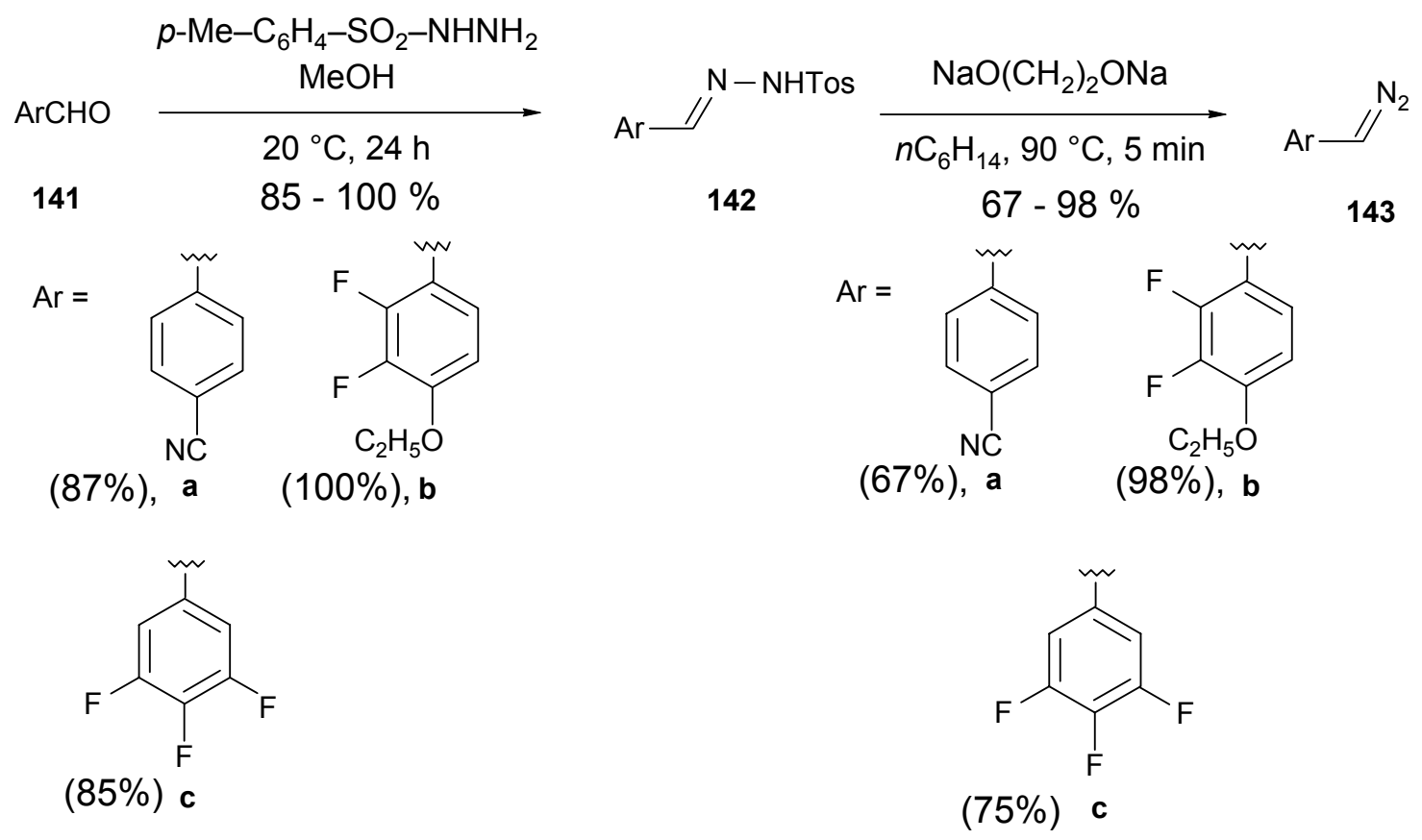

Schema 41. Darstellung von Aryldiazomethan 143 über Tosylhydrazone 142.

Die Kondensation der Aldehyde 141 mit Tosylhydrazin liefert in hohen Ausbeuten die erwünschten Zielprodukte 142. Die Synthese der fluorierten Phenyldiazomethanderivate 143b und 143c versprechen einen Zugang zu den erwünschten fluorierten mesogenen Substanzen der Klassen 42-44. 


\section{Konvergente Synthese neuer flüssigkristalliner Substanzen mit zentraler Bicyclo[3.1.0]hexan-Einheit}

\subsection{Vorüberlegungen}

Nach dem Scheitern der intramolekularen Variante der Synthese von exo-6-Arylbicyclo[3.1.0]hexanderivaten 57 bleibt als einzige Methode zur Synthese von exo,anti-3,6disubstituierten Bicyclo[3.1.0]hexan-Derivaten die Addition von Carbenen oder Carbenoiden an die olefinische Doppelbindung entsprechender 4-substituierter Cyclopentenderivate.

Zur Erzeugung von Carbenen oder Carbenoiden ${ }^{[77]}$ existieren verschiedene Varianten. Zur Wahl steht die homogene Übergangsmetall-katalysierte Zersetzung von Diazoverbindungen, bei der alleine in den letzten 30 Jahren eine nahezu unermessliche Vielfalt an Varianten bezüglich der Metalle ${ }^{[78]}$ und Liganden ${ }^{[79]}$ in Katalysatoren, der Katalysatormenge, der Reaktionstemperaturen und von Lösungsmitteln veröffentlicht wurde.

Wenn aus berechtigten Überlegungen und aus experimenteller Erfahrung die Carbenoidaddition mittels katalytischer Zersetzung von Diazoverbindungen fehlschlägt, ist die photochemische Erzeugung von Carbenen mit nachfolgender Addition ein alternativer ${ }^{[80]}$ Weg. Die Existenz freier Carbene könnte darüber hinaus die Additionsneigung der Carbenkomponente an die Doppelbindung der Cyclopentenderivate erhöhen.

Eine weitere Methode, die einiges an synthetischem Geschick verlangt, ist die Addition von Chlorcarbenen. Die Vorstufen von Chlorcarbenen können aus Aldehyden durch Umsetzung mit Phosphorpentachlorid erzeugt werden. In vielen Fällen ist die Addition von Chlorcarbenen eine bewährte Methode. 


\subsection{Die Erzeugung und Versuche zur Addition von Chlorcarbenen}

Die Addition von aus Aryldichlormethanderivaten erzeugten Carbenen an olefinische Doppelbindungen ist seit langem bekannt. Besonders $\mathrm{zu}$ nennen sind die Arbeiten von McElvain und Weyna, die zur Erzeugung der Halogencarbenoide Kalium-tertbutanolat ${ }^{[81]}$ eingesetzt haben, sowie Moss ${ }^{[82]}$, der für die Carbenerzeugung Methyllithium benutzt hat. Die Stereochemi $^{[83]}$ der mit Cyclohexen resultierenden Produkte wurde schon sehr früh untersucht. Dabei wurde festgestellt, dass bei Carbenoidadditionen der beabsichtigten Art beide Diastereomere in unterschiedlichen Verhältnissen entstehen. Die 1964 von Hodgkins et al. ${ }^{[84]}$ durchgeführten stereochemischen Untersuchungen bei der Addition des durch Einwirkung von Kalium-tert-butylat auf Phenyldichlormethan erzeugten Phenylchlorcarbens an Cyclohexen ergaben mit mäßiger Ausbeute das 7-Chlor-7-phenylnorcaran (7-Chlor-7phenylbicyclo[4.1.0]heptan). Nachfolgende Reduktion mit Zink und 5\% Natriumethanolat ergab überwiegend das thermodynamisch stabilere exo-Diastereomer 147a.

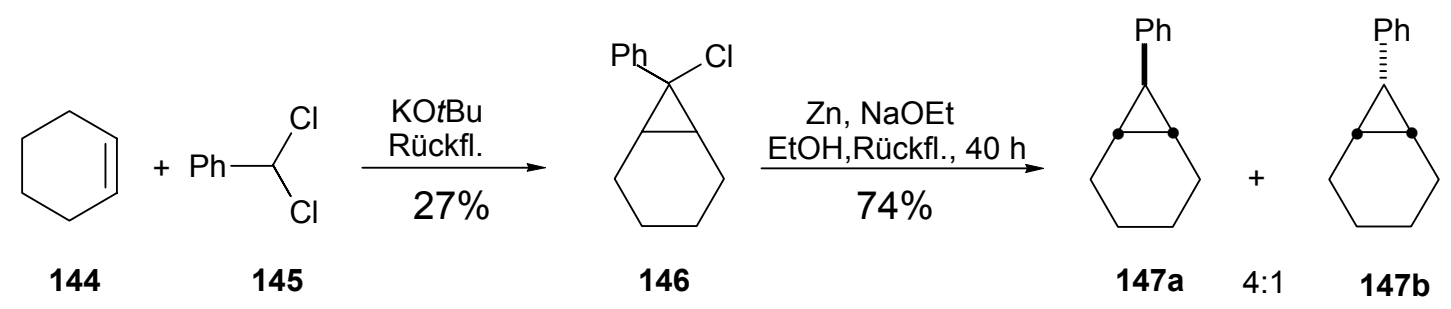

Schema 42. Synthese der 7-Phenylnorcarane 147.

1967 berichteten Cope et al. ${ }^{[85]}$ erstmals über die Addition des aus Benzalchlorid 145 erzeugten Phenylchlorcarbens an Cyclohepten. Radikalische Dechlorierung mit Triphenylzinnhydrid ergab ein Gemisch von exo- und endo-8-Phenylbicyclo[5.1.0]octan im Verhältnis 6:1. Erstaunlicherweise ist eine analoge Reaktionfolge an Cyclopenten nicht berichtet worden. Für die vorliegende Problemstellung böte die Addition eines Arylchlorcarbens an ein geeignetes 4-substituiertes Cyclopenten den Zugang zu den Zielmolekülen des Typs 42-44. Die neuere Methode zur Erzeugung von Chlorcarbenen unter Verwendung von LTMP wurde nicht berücksichtigt. 


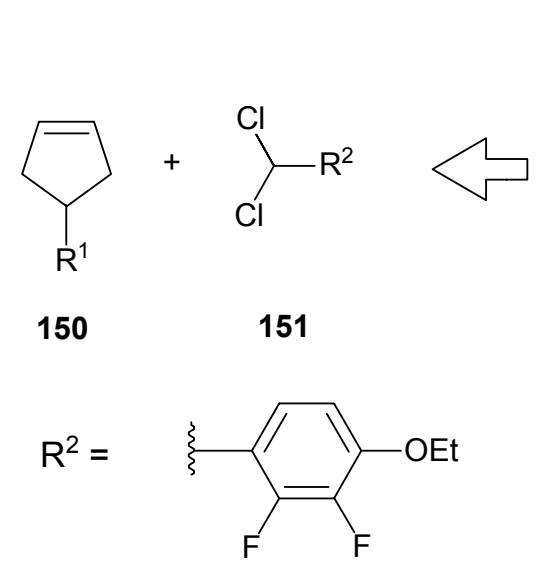

a

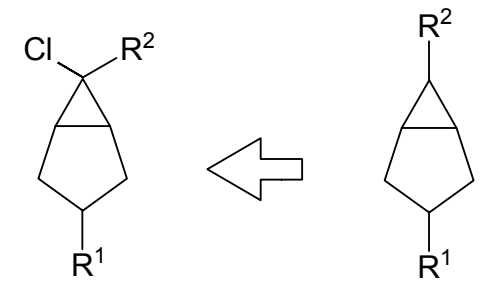

149

148

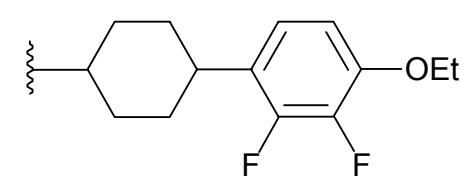

b

Schema 43. Retrosynthetische Überlegungen zu exo-6-Aryl-3-alkylbicyclo[3.1.0]hexanen.

Um diese Idee zu verwirklichen, wurde versucht, unter den literaturbekannten Bedingungen die aus dem Aryldichlorcarben 151 erzeugten Carbene an das 4- $n$-Pentylcyclopenten 150 zu addieren. Dazu wurden die in nicht reiner Form angefallenen Aryldichlormethanderivate 151a und 151b in 4- $n$-Pentylcyclopenten als Lösungsmittel bei Raumtemperatur mit Kalium-tertbutylat behandelt und nach ausbleibender Reaktion auf $50{ }^{\circ} \mathrm{C}$ erwärmt. In beiden Versuchen konnten die erwarteten 6-substituierten 6-Chlor-3-n-pentylbicyclo[3.1.0]hexan-derivate 149 nicht nachgewiesen werden. Wegen der nur in knappem Umfang zur Verfügung stehenden Difluorarenderivate zur Herstellung der entsprechenden Aryldichlormethanderivate 151 und der ohnehin literaturgemäß nicht allzu vielversprechenden Ausbeuten wurden weitere Versuche zur Addition von Arylchlorcarbenen an 4- $n$-Pentylcyclopenten nicht unternommen.

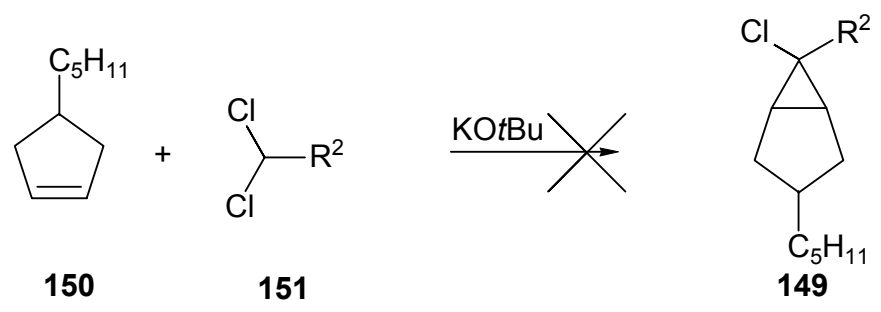

Schema 44. Versuch zur Darstellung von 6-Aryl-6-chlorbicyclo[3.1.0]hexanderivaten. 


\subsection{Katalysierte Additionen von Phenyldiazomethanderivaten}

\subsubsection{Allgemeine Vorüberlegungen über geeignete Katalysatoren}

Obwohl für die katalysierte Addition von Diazomethan an olefinische Doppelbindungen seit mehr als 50 Jahren experimentelle Ergebnisse und mechanistische Betrachtungen veröffentlcht sind, ist die Addition von Aryldiazomethanderivaten an olefinische Doppelbindungen sehr wenig untersucht. Eine Beilstein-Crossfire-Recherche nach der Addition von Aryldiazomethanderivaten an 1,2-disubstituierte Olefine ergab 23 Literaturstellen, von denen der überwiegende Teil auf photolytische Addition von Aryldiazomethan und Zinkhalogenid-katalysierte Addition von Arylchlorcarbenen an Olefine durch Closs et al. ${ }^{[86][87]}$ zurückzuführen ist.

Dabei sind für die Zersetzung von Diazoverbindungen und anschließende Addition der entstehenden Carbene oder Carbenkomplexe mehrere verschiedene Katalysatoren bekannt.

Die Untersuchungen umfassen sowohl den Zerfall von Diazomethan an Metalloberflächen $(\mathrm{Ni}, \mathrm{Pd}, \mathrm{Cu}, \mathrm{Fe}, \mathrm{Co}, \mathrm{Ru}, \mathrm{U})^{[88][89]}$, sowie die homogen und heterogen katalysierte Bildung von Carbenen. Unter den best untersuchten Katalysatoren sind Kupfer-, Palladium- und Rhodiumderivate zu nennen. Unter den Kupfersalzen sind Kupfer(II)chlorid, Cu(acac) 2 , und $\mathrm{Cu}\left(\mathrm{OSO}_{2} \mathrm{CF}_{3}\right)_{2}$ sowie deren Komplexe mit chiralen Liganden, wie Semicorrine ${ }^{[90]}$, 5Azasemicorrine ${ }^{[91]}$, und Bipyridine ${ }^{[92]}$ untersucht. Da in den hier durchgeführten Versuchen weder die Edukte noch die Zielmoleküle chiral sind, wurde eine Verwendung von chiralen Katalysatoren garnicht in Erwägung gezogen. Je nach verwendetem Olefin werden Ausbeuten zwischen 5 und 90\% berichtet. Bei der Cyclopropanierung von Cyclohexen, Cyclohepten und Cycloocten wird eine von 20 auf 80\% zunehmende Ausbeute an Cyclopropanierungsprodukt mit zunehmender Ringgröße berichtet ${ }^{[93]}$. Die Kupferderivate bieten den Vorteil, dass sie seit langem bekannt, gut untersucht und entweder günstig zu kaufen oder jedenfalls einfach herzustellen sind. Im Allgemeinen werden Kupferderivate in katalytischen Mengen von 5-10 mol\% eingesetzt.

Kirmse et al. ${ }^{[94]}$ untersuchten die die Cyclopropanierung von cyclischen Olefinen mit Diazomethan mittels Bis[ $\eta$-allylpalladiumchlorid] und Kupferhalogeniden und fanden eine von Kupferhalogeniden verschiedene Stereoselektivität bei der Bildung der Cyclopropanderivate. Auch mit anderen Palladiumkatalysatoren sind Cyclopropanierungen an cyclischen Olefinen bekannt ${ }^{[95]}$. Wie bei den analogen Kupfer-katalysierten Reaktionen nehmen Ausbeuten an Bicyclo[n.1.0]alkanderivaten unter Palladiumkatalyse mit 
zunehmender Ringgröße von 10\% im Falle des Cyclohexens bis 92\% im Falle des Cyclooctens $^{[96]}$ zu. Die Palladiumliganden scheinen im Fall der Cylopropanierung von n-Alkenen keinen größeren Einfluß auf die Ausbeuten zu haben. Palladiumkatalysatoren werden in Mengen von $0.1-10 \mathrm{~mol} \%$ eingesetzt.

Rhodiumkomplexe sind in neuerer Zeit eingehend hinsichtlich ihrer katalytischen Fähigkeiten zur Zersetzung von Diazoverbindungen ${ }^{[97]}$ untersucht worden. Im Falle der EthyldiazoAcetataddition an Doppelbindungen sind sie in ihrer Effektivität unerreicht. Der zweikernige Rhodiumkomplex eröffnet hinsichtlich Selektivität und Steuerung der Reaktivität wesentlich größere Möglichkeiten als es für die einkernigen Kupfer- oder Palladiumkomplexe möglich ist. Die Verwendung von dimerem Rhodiumacetat wird hier besonders in Erwägung gezogen, weil mindestens ein Fall bekannt ist, in dem Rhodiumacetat eine zu Palladiumacetat komplementäre Selektivität aufweist.

\subsubsection{Katalysierte Additionen von Aryldiazomethanderivaten an 4-Alkylcyclopentene}

Da die katalysierte Zersetzung von Aryldiazomethanderivaten mit nachfolgender Carbenaddition an olefinische Doppelbindungen erstaunlicherweise nicht intensiv untersucht ist, wurden die bekanntesten Katalysatoren für die Additionsreaktion von Diazomethan in der Reaktion von Aryldiazomethanderivaten mit den 4-substituierten Cyclopentenen erprobt.

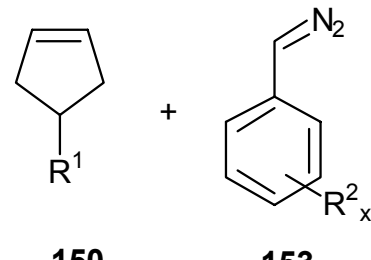

150
153

Schema 45. Retrosynthetische Überlegungen $\quad$ zu 3-n-Alkyl-6-

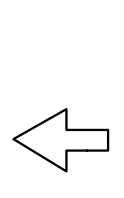

Schema 45. Retrosynthetische Überlegungen $\quad$ zu 3-n-Alkyl-6-

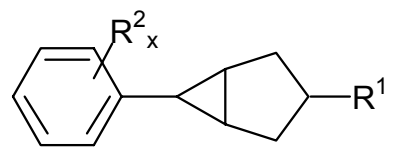

152 arylbicyclo[3.1.0]hexanderivaten 152.

Um von einer möglichst einfachen und in großem Umfang erhältlichen Substanz auszugehen, wurde zunächst 4- $n$-Pentylcyclopenten unter Rhodiumacetat-katalyse mit Phenyldiazomethan umgesetzt. Unter diesen Bedingungen konnte jedoch nur Stilben als Dimerisierungsprodukt des Phenyldiazomethans isoliert werden. Daraus wurde geschlossen, dass Rhodiumacetat für diese Reaktionen ungeeignet ist.

Da das das 4- $n$-Pentylcyclopenten 103 in ausreichender Menge synthetisiert werden konnte, 
wurde es mit dem ebenfalls in ausreichender Menge vorhandenen (4-Cyanophenyl)diazomethan $\mathbf{1 5 4}$ in Gegenwart von Palladiumacetat umgesetzt. Nach säulenchromatographischer Reinigung konnte $30 \%$ eines Gemisches der beiden Diastereomere 155a und 155b isoliert werden, deren Trennung mittels präparativer HPLC gelang Das Verhältnis von endo- zu exo-Derivat betrug 1:6.<smiles>CCCCCCCCCCC</smiles><smiles>N#Cc1ccc(C=N)cc1</smiles>

103
154

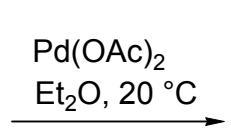

$30 \%$<smiles>[CH2]C1C[C@@H]2C(c3ccc(C#N)cc3)[C@H]2C1</smiles>

$155 a$

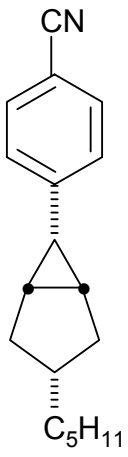

$155 b$

Schema 46. Palladiumkatalysierte Addition von (4-Cyanophenyl)diazomethan an 4- $n$-Pentylcyclopenten.

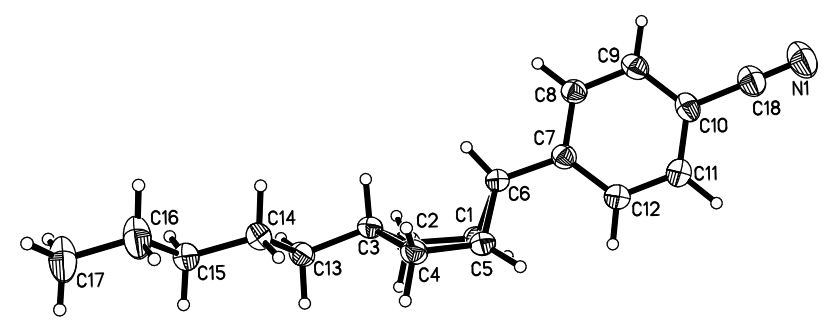

$155 a$

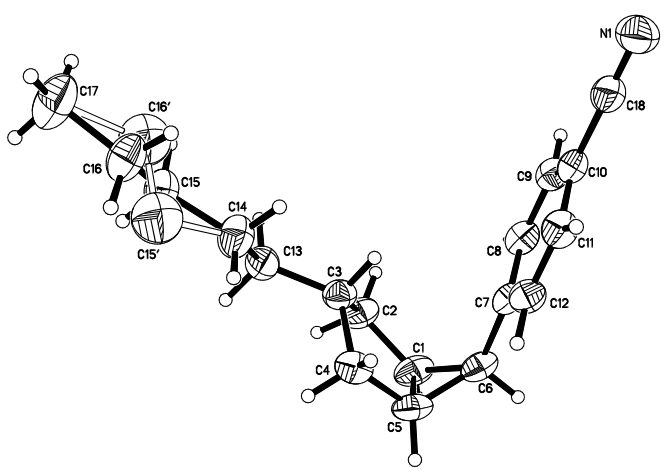

$155 b$

Abb. 17. Strukturen der Verbingungen 155a und 155b im Kristall.

Die Konstitutionen und Konfigurationen von 155a und 155b wurden durch RöntgenStrukturanalysen bestätigt. Ein Vergleich von 155b mit bekannten „BananenFlüssigkristallen“ vom Typ 156a ${ }^{[98]}$ oder $\mathbf{1 5 6} \mathbf{b}^{[99]}$ zeigt zwar eine gewisse geometrische Ähnlichkeit, wegen der kurzkettigen Substituenten und der sterisch anspruchsvollen Geometrie des Bicyclo[3.1.0]hexan-Mittelstücks besteht allerdings nur geringe Hoffnung dass 155b eine Mesophase bildet. Wegen der geringen vorhandenen Substanzmenge konnte wurde 155b nicht auf seine flüssigkristallinen Eigenschaften untersucht werden. Das 
exo-Diasereomere 155a weist dagegen vielversprechende Eigenschaften auf.

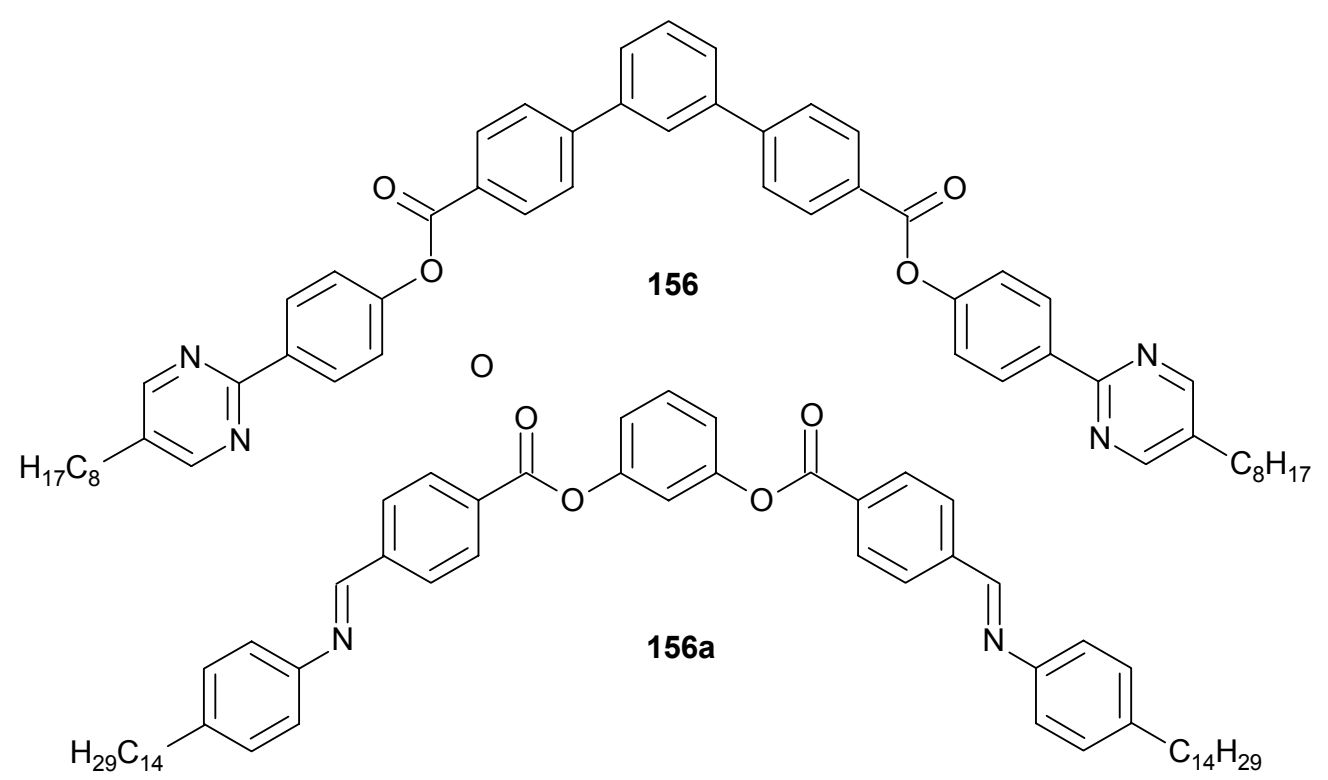

Abb. 18. Beispiele für Bananen Flüssigkristalle.

Nach der erfolgreichen Synthese der Verbindung 155a wurde versucht, eine analoge Reaktion mit den Aryldiazomethanderivaten 157, 158 und 159 durchzuführen.<smiles>N=Cc1cc(F)c(F)c(F)c1</smiles>

157<smiles>CCOc1ccc(C=N)c(F)c1F</smiles>

158

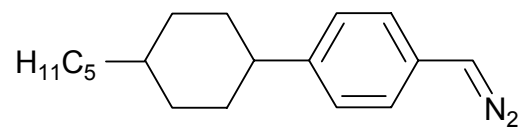

159

Abb. 19. Fluorierte und Cyclohexylsubstituierte Aryldiazomethanderivate.

Die in Flüssigkristallen häufig anzutreffenden fluorierten Arylreste wie in $\mathbf{1 5 7}$ und $\mathbf{1 5 8}$ sollten wegen der stark elektronenziehenden Substituenten die aus den Diazoverbingungen gebildeten Carbene besonders elektrophil machen wie dasjenige aus (4Cyanophenyl)diazomethan (154). Doch führten die Reaktionen unter den für 154 benutzen Bedingungen nicht zu den Carbenaddukten an das Cyclopentenderivat, sondern ausschließlich zur Bildung der Stilbene durch Carbendimerisierung. 
Bei der Umsetzung von 159 mit dem 4-n-Pentylcyclopenten 103 entstand zwar wie anhand der NMR- und Massenspektren geschlossen werden konnte, in geringem das erwünschte Bicyclo[3.1.0]hexan-Derivat, doch ließ es sich nicht von dem Dimerisierungsprodukt des Carbens 159 abtrennen.

\subsection{Photochemische Spaltung von Aryldiazomethanderivaten}

Für die photolytische Erzeugung von Phenylcarben aus Phenyldiazomethan und dessen nachfolgende Addition an Olefine haben Closs et al. ${ }^{[86]}$ ebenfalls an cis-2-Buten verwendet, um die Stereoselektivität dieser Reaktion zu ermitteln. Für die hier in Betracht gezogene Addition an das Cyclopentenderivat $\mathbf{1 0 3}$ spielt jedoch die cis-trans-Isomerisierung keine Rolle.

Bei einstündiger Belichtung mit einer $450 \mathrm{~W}$ Quecksilberdampflampe in einer gekühlten Photolyseapparatur verschwand die rote Farbe der Diazokomponente 158 nicht. Nach Entfernen des Lösungsmittels und der Olefinkomponente im Vakuum zeigten sich im NMRSpektrum der Rohsubstanz keine erwarteten Cyclopropylsignale. Verlängern der Bestrahlungszeit auf 12 Stunden führte dagegen zum Verblassen der roten Aryldiazomethanfärbung der Lösung. Als einziges Reaktionsprodukt fiel ein farbloses, in Pentan, Dichlormethan und THF sehr schlecht lösliches Polymer an. Das erwünschte Additionsprodukt $\mathbf{1 6 0}$ konnte trotz mehrfacher Versuche nicht isoliert werden. Möglicherweise wäre die photolytische Addition der Carbene zu realisieren, wenn das Olefin als Lösungsmittel eingesetzt werden könnte. Da die entsprechenden 4-substituierten Cyclopentene 103 zuvor aufwendig in Kupplungsreaktionen dargestellt wurden, stand für einen Versuch, der eine erhebliche Menge der Olefinkomponente benötigte, nicht genug Substanz zur Verfügung. 


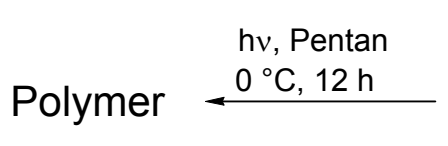

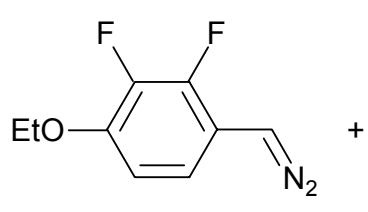

158<smiles>CCCCCCCCCCCC</smiles>

103

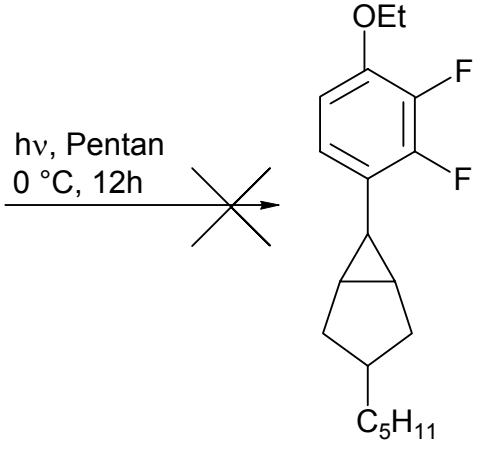

160

Schema 47. Versuch der photolytischen Carbenerzeugung mit nachfolgender Addition.

\subsection{Addition von tert-Butyldiazoacetat mit nachfolgender Homologisierung}

Bei der Erzeugung der Diester 35a und 35b wurde unter Rhodiumacetat-Katalyse mit guter Ausbeute Ethyldiazoacetat an den Cyclopent-4-encarbonsäure-tert-butylester 32 addiert. Eine analoge Addition von tert-Butyldiazoacetat unter Rhodiumoctanoat-Katalyse an die 4-substituierten Cyclopentenderivate 161 sollte die Bicyclo[3.1.0]hexanderivate mit höherer exo-Selektivität ergeben. Durch nachfolgende Reduktion der Estergruppe, Transformation der Hydroxymethylgruppe und anschließende Kreuzkupplung mit einem Organometallderivat sollte man literaturgemäß in guten Ausbeuten zu den Zielmolekülen 165 gelangen.<smiles>[R]C1CC=CC1</smiles>

161

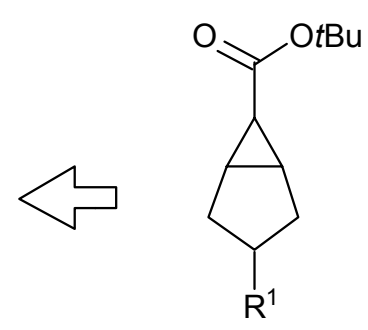

162

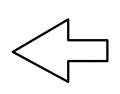

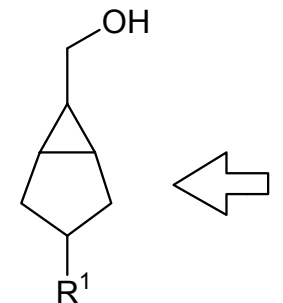

163

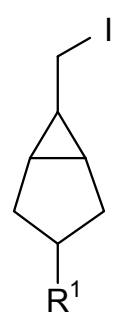

164

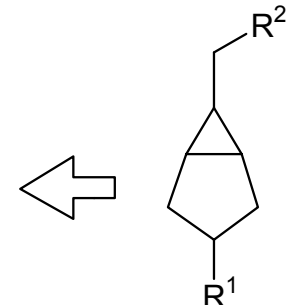

165

Schema 48. Retrosynthese von 6-n-Alkyl-3-alkylbicyclo[3.1.0]hexanderivaten und 6-Aryl-3-alkylbicyclo[3.1.0]hexanderivaten.

Gemäß der seit neuerem bekannten und im Vergleich zur Rhodiumacetat-katalysierten Addition von Ethyldiazoacetat selektiveren Rhodiumoctanoat-katalysierten Addition von tertButyldiazoacetat an Olefine wurden im Falle der hier untersuchten Reaktion mit 4substituierten Cyclopentenderivaten Ausbeuten zweier Diastereomere zwischen 62 und 86\% erzielt. Da in vorherigen Experimenten die Isomerisierung von analogen Cyclopropancarbonsäureestern nicht gelungen ist, ist die Strukturaufklärung des tert-Butylesters 162a für 
die Stereochemie der nachfolgenden Produkte äußerst wichtig. Da die Alkylsubstituenten wie gewöhnlich eine Konformationsanalyse mittels NMR-Spektroskopie erschweren, ergibt die Röntgenstrukturanalyse die gewünschten Informationen.

Die Diastereomere 162a und 162b lassen sich entweder chromatographisch oder durch Kristallisation trennen und ergeben die erwünschten exo,exo-3,6-disubstituierten Bicyclo[3.1.0]hexane 162 in 39-48\% Ausbeute. Die nachfolgende Reduktion der tert-Butylester mit Lithiumaluminiumhydrid verläuft in allen Fällen quantitativ. Nach einem Protokoll von Nicolaou ${ }^{[52]}$ kann aus den resultierenden Alkoholen das entsprechende exo-6(Jodmethyl)bicyclo[3.1.0]hexanderivat 164 mit Ausbeuten von 91-99\% hergestellt werden. Die danach erfolgte lithiumcupratvermittelte Kreuzkupplung mit Alkylmagnesiumbromiden liefert die gewünschten Zielverbindungen 165 mit Ausbeuten von 77-85\%.

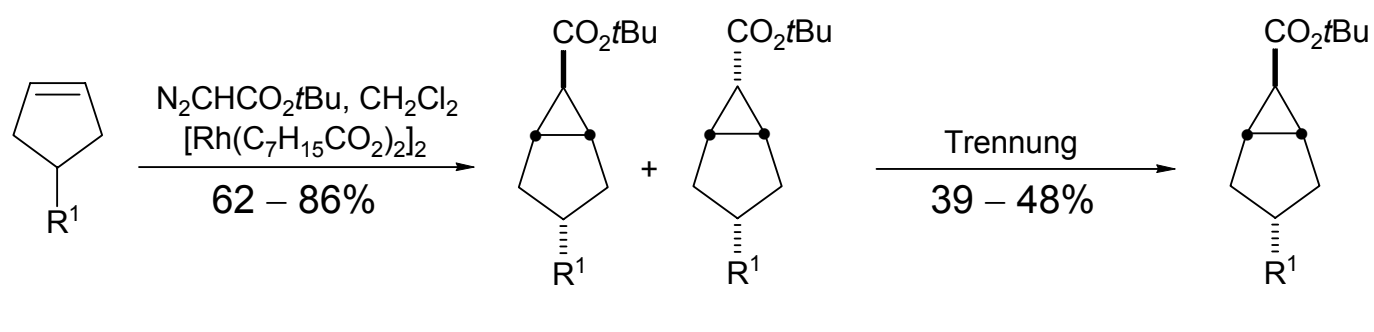

161

$$
\text { exo-162 }
$$

endo-162b

exo-162

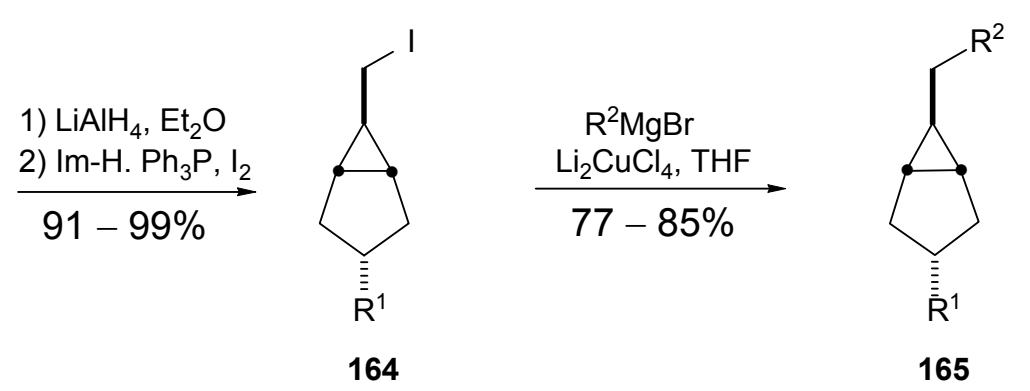

Schema 49. Synthese von exo,exo-3,6-disubstituierten Bicyclo[3.1.0]hexanderivaten. Details siehe Tabelle 4. 


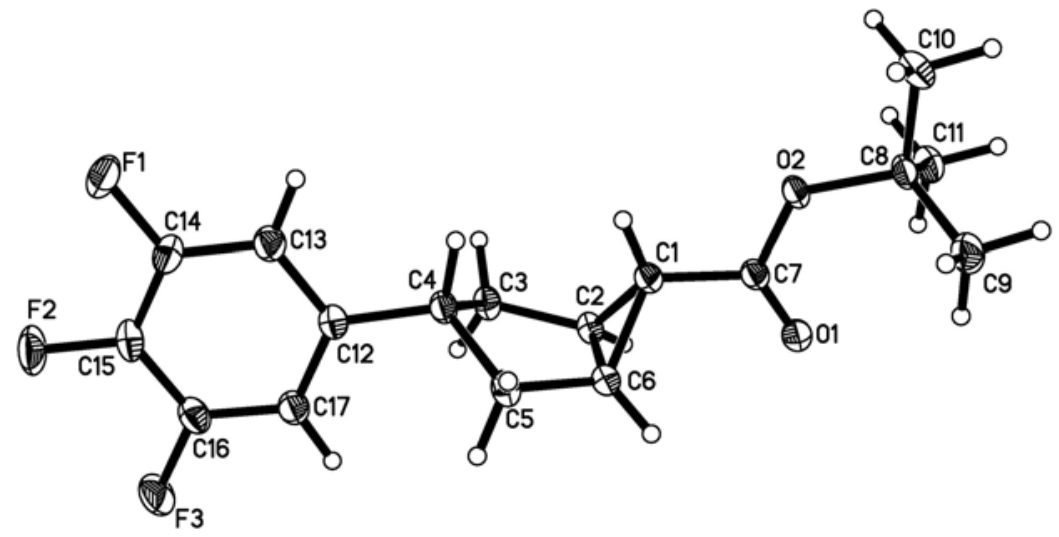

Abb. 20. Struktur des 3-(3,4,5-Trifluorphenyl)bicyclo[3.1.0]hexan-6-carbonsäure-tertbutylesters im Kristall. 
Tabelle 4. Synthesen neuer Substanzen mit zentraler Bicyclo[3.1.0]hexan-Einheit

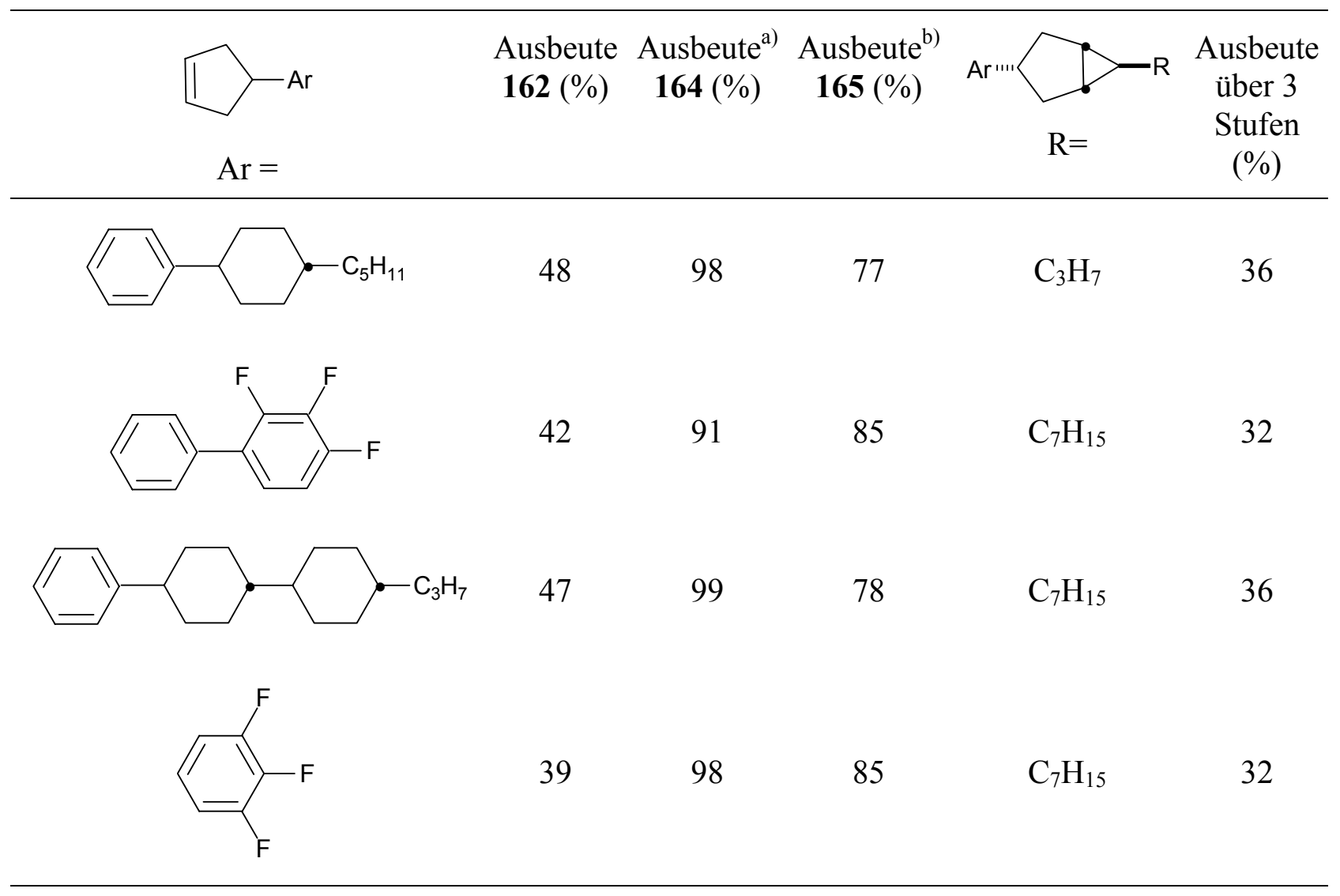

Die Strukturen der synthetisierten Substanzen 162a-1 und 162b im Festkörper konnten mit Röntgenstrukturanalyse bestimmt werden. ${ }^{\text {a) }}$ : Reduktion und Jodierung; b): Kreuzkupplung. 


\section{Diskussion der Ergebnisse: Synthetische Resultate und physikalische Eigenschaften der Produkte}

\section{Neue Bausteine zur Synthese flüssigkristalliner Substanzen}

Nach verschiedenen Synthesestrategien konnten die in Schema 50 abgebildeten Olefine in für weitere Synthesen ausreichender Menge dargestellt werden. Die anfänglichen Probleme der begrenzten Verfügbarkeit der Cyclopentencarbonsäure und des Cyclopentenols konnten behoben werden.

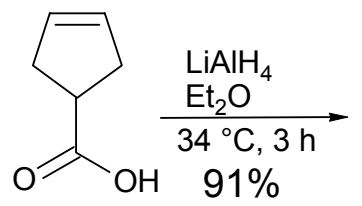

31<smiles>OCC1CC=CC1</smiles>

94

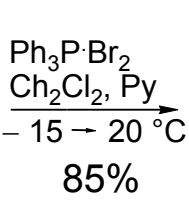

$85 \%$<smiles>COC(=O)C1CC=CC1</smiles>

109<smiles>O=C(O)C1CC=CC1</smiles>

31<smiles>COC(=O)C1CC=CC1</smiles>

109

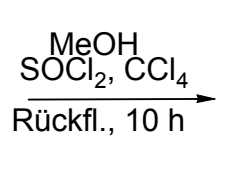

quant.<smiles>BrCC1CC=CC1</smiles>

95
2) $\mathrm{ArCH}_{2} \mathrm{I}$

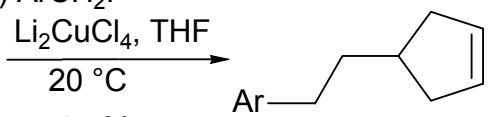

100

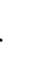


3-nAlkyl-6-Aryl-bicyclo[3.1.0]hexan (44) hergestellt werden. Durch Addition von Ethyldiazoacetat bzw. tert-Butyldiazoacetat an die Olefine mit nachfolgender Reduktion der Ester, Jodierung der Alkohole und übergangsvermittelter Kreuzkupplung wurden 6- $n$-alkylsubstituierte Substanzen vom Typ 45 synthetisiert.

Es hat sich weiterhin gezeigt, dass Ringschlussreaktionen über ein 3-substituiertes Pentan1,5-diyl-bis-magnesiumbromidderivat in zufriedenstellender Ausbeute Cyclohexanolderivate vom Typ 168 bilden, deren Überführung in das Fluorid oder Defunktionalisierung der Hydroxyfunktionalität noch nicht näher untersucht wurde. Diese Reaktion könnte aber Synthesen der Substanzklassen 40-42 erlauben.<smiles>[R1]C1CC2C(Br)C2C1</smiles>
nAlkyl $(102-104)$ $\mathrm{R}=$ Aryl $(89-93)$

Diastereomeren-$$
\mathrm{R}=\mathrm{Ar}
$$$$
\text { nAlkyl (102 - 104) }
$$<smiles>CC(C#N)C#N</smiles><smiles>[R]C1CC=CC1</smiles>

$\mathrm{N}_{2} \mathrm{CHCO}_{2} \mathrm{R}$

Kat.

Diastereomerentrennung

166

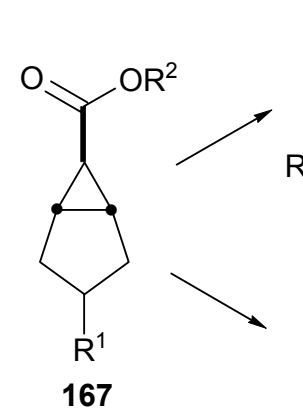

167<smiles></smiles>

168

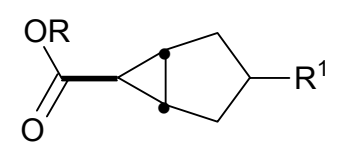

169

Schema 51. Darstellungsmöglichkeiten für 3,6-disubstituierte Bicyclo[3.1.0]hexane. 


\section{Eigenschaften der neuen Substanzen mit zentraler Bicyclo[3.1.0]hexan-Einheit}

Im Rahmen der in der vorliegenden Arbeit durchgeführten Synthesen konnten exemplarisch sieben Testsubstanzen der Typen 43 - 45 sowie des zunächst nicht in Erwägung gezogenen Typs 172 in den beschriebenen konvergenten Synthesen dargestellt, strukturell abgesichert und hinsichtlich ihrer physikalischen Eigenschaften untersucht werden. Weiterhin konnten neue Ausgangsmaterialien für die bislang noch nicht synthetisierten 3,6-Dialkylbicyclo[3.1.0]hexane der Typen $\mathbf{4 0}$ - 42 dargestellt werden.

Die physikalischen Eigenschaften der neuen Verbindungen sind in Tabelle 5 zusammengestellt.<smiles>CC(C)C1[C@H]2CC(c3cc(F)c(F)c(F)c3)C[C@H]12</smiles>

170

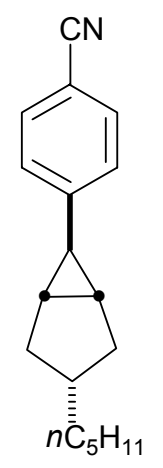

171

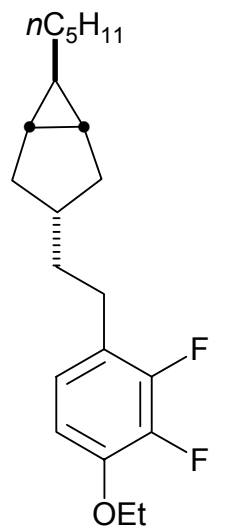

172<smiles>CC(C)C1C2CC(c3ccc(-c4cc(F)c(F)c(F)c4)cc3)CC21</smiles>

173

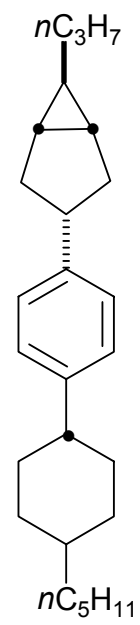

174

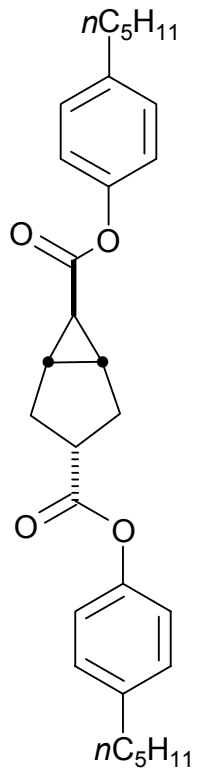

175

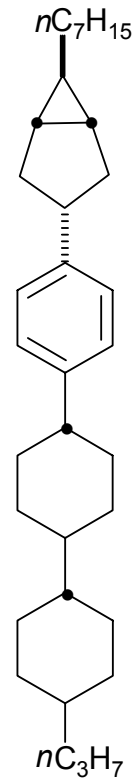

176

Abb. 21. Neue potentiell flüssigkrisalline Bicyclo[3.1.0]hexanderivate. 
Tabelle 5: Physikalische Eigenschaften der neuen Bicyclo[3.1.0]hexan-Derivate

\begin{tabular}{ccccc}
\hline Substanz & Phasenübergänge & $\Delta \varepsilon$ & $\Delta \mathrm{n}$ & $\eta[\mathrm{mPaS}]$ \\
\hline 169 & C 12.8 I & $1.4^{\text {a) }}$ & $-0.090^{\text {a) }}$ & $16.5^{\text {a) }}$ \\
170 & C $<20^{\circ} \mathrm{C} \mathrm{I}$ & $-3.46^{\text {a) }}$ & $0.020^{\text {a) }}$ & - \\
171 & C 33.8 N 46.7 I & $14.0^{\text {a) }}$ & $0.124^{\text {a) }}$ & - \\
172 & C 30.1 I & $10.1^{\text {a) }}$ & $0.090^{\text {a) }}$ & $52.0^{\text {a) }}$ \\
173 & C 18.5 SmB 107.9 I & $0.3^{\text {a) }}$ & $0.070^{\text {a) }}$ & $23.3^{\text {a) }}$ \\
174 & C 60.5 N 86.4 I & $0.5^{\text {a) }}$ & $0.070^{\text {a) }}$ & - \\
175 & C 36.9 SmX 246.7 I & $-0.6^{\text {b) }}$ & $0.117^{\text {b) }}$ & $61.3^{\text {b) }}$ \\
\hline
\end{tabular}

a) Extrapoliert: 15\% in der Basismischung auf Benzonitrilbasis Merck ZLI-1132®.

b) Extrapoliert: 5\% in der Basismischung auf Benzonitrilbasis Merck ZLI-1132®.

Wie anfangs besprochen, sind die Eigenschaften von flüssigkristallinen Substanzen unter anderem von der Molekülform abhängig. Da die entsprechenden Vergleichssubstanzen nicht alle kommerziell erhältlich sind, wurde der Diester 39 im Chisso Specialty Chemical Research Center nach einer Literaturvorschrift von Neubert ${ }^{[100]}$ synthetisiert.

Ein Vergleich mit den bekannten isomorphen Substanzen mit Cyclohexanmittelstück ergibt, dass die neuen Bicyclo[3.1.0]hexanmittelstücke die Übergangstemperatur erniedrigen. Die dielektrischen und optischen Anisotropien $\Delta \varepsilon$ und $\Delta \mathrm{n}$ der neuen Substanzen sind mit denen der Cyclohexanderivate vergleichbar, zeigen aber tendenziell, wie durch die Gleichungen von Maier und Meier ${ }^{[101]} \mathrm{zu}$ erwarten, etwas höhere Werte als die isomorphen Vergleichssubstanzen. Für weitere Analysen und Tests hinsichtlich der Mischbarkeit, Viskosität und dem Verhalten der Substanzen in den üblichen flüssigkristallinen Mischungen zur technischen Anwendung müssten einige Gramm der jeweiligen Verbindung synthetisiert werden, was mit den hier beschriebenen Methoden praktikabel ist. 


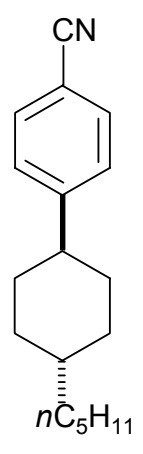

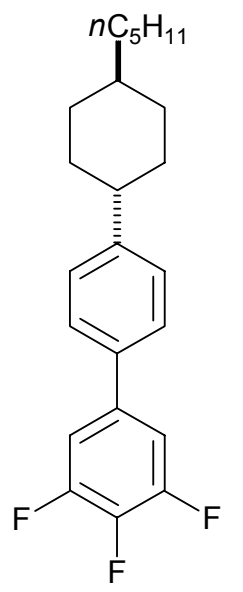

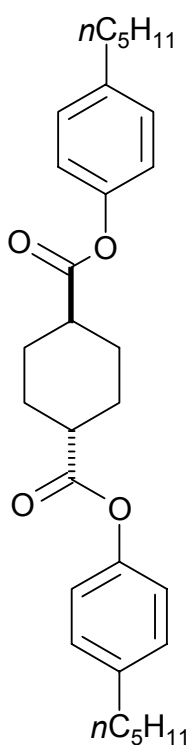

39

Abb. 22. Vergleichssubstanzen zur Beurteilung der mesogenen Eigenschaften.

Tabelle 6. Physikalische Eigenschaften der bekannten Vergleichssubstanzen

\begin{tabular}{ccccc}
\hline Substanz & Phasenübergänge & $\Delta \varepsilon$ & $\Delta \mathrm{n}$ & $\eta[\mathrm{mPaS}]$ \\
\hline 177 & N 53.0 I & $11.0^{\text {a) }}$ & $0.118^{\text {a) }}$ & - \\
178 & C 30.4 N 58.0 I & $10.3^{\text {a) }}$ & $0.124^{\text {a) }}$ & 44.3 \\
39 & C 95.1 SA 131.1 N 155.3 I & $-1.1^{\text {b) }}$ & $0.117^{\text {b) }}$ & -
\end{tabular}

a) Extrapoliert: 15\% in der Basismischung auf Benzonitrilbasis Merck ZLI-1132®.

b) Extrapoliert: $5 \%$ in der Basismischung auf Benzonitrilbasis Merck ZLI-1132®. 


\section{Zusammenfassung}

In den letzten Jahren wurde durch die Kulinkovich - de Meijere Reaktion ${ }^{[21][22]}$ eine Möglichkeit geschaffen und optimiert, in einer einstufigen Synthese aus einem Cycloalken und Dialkylformamiden (n+3)-Aminobicyclo[n.1.0]alkane herzustellen. Daraus entstand zunächst die Idee, ein 3-Azabicyclo[3.1.0]hex-6-ylamin als zentrale Einheit in mesogenen Substanzen zu erproben. Nachdem es gelungen war, einige Substanzen vom Struktrutyp 18 herzustellen und auf ihr mesogenes Potential zu untersuchen, wurde beschlossen, die Eigenschaften der neuen Substanzklasse dadurch zu verbessern, dass die Heteroatome weitgehend aus der Grundstruktur entfernt werden sollten, um die Löslichkeit der Substanzen in bekannten flüssigkristallinen Basismischungen zu erhöhen sowie die Photolabilität, die Oxidations- und Hydrolyseempfindlichkeit zu beheben.

Im Rahmen der vorliegenden Arbeit wurden auf verschiednen Wegen die 3,6-disubstituierten Bicyclo[3.1.0]hexanderivate 35a und 170 - 176 synthetisiert, strukturell abgesichert und auf ihre physikalischen Eigenschaften hin untersucht. Einige der neuen Substanzen weisen gute flüssigkristalline Eigenschaften auf. Als Trend ist erkennbar, dass erwartungsgemäß die Übergangstemperaturen aus der kristallinen Phase niedriger liegen als die der isomorphen Vergleichssubstanzen. Auch der mesogene Temperaturbereich ist breiter, als die der bekannten Mesogene mit 1,4-disubstituierten Cyclohexaneinheiten. Im Vergleich zu den bekannten gebräuchlichen Substanzen ist feststellbar, dass dielektrische Anisotropie der neuen Substanzklasse häufig niedriger und die optische Anisotropie in ähnlichen Bereichen liegt (vgl. Tabelle 5 und 6).

Um weitere Aussagen über den technischen Nutzen der neuen Bicyclo[3.1.0]hexane machen zu können, müssten noch wesentlich mehr Substanzen hergestellt werden, sodass man eine eutektische Mischung aus Mesogenen herstellen könnte. Die erwarteten Vorteile wären besonders eine wesentlich erniedrigte Temperatur für den Übergang aus der kristallinen Phase sowie ein breiterer Temperaturbereich für die Mesophase. In Anbetracht der vergleichsweise leichten Synthetisierbarkeit der Flüssigkristalle mit zentralen Cyclohexaneinheiten ist jedoch fraglich, ob unter wirtschaftlichen Aspekten diese neue Substanzklasse wegen ihrer physikalischen Eigenschaften die bekannten Eutektika ersetzen kann. 


\section{E. Experimenteller Teil}

\section{Allgemeines}

${ }^{1}$ H-NMR: Bruker AM 250 (250 MHz); $\delta$ = 0 für Tetramethylsilan, 7.26 für Chloroform, 7.16 für $\mathrm{C}_{6} \mathrm{D}_{5} \mathrm{H}$. Charakterisierung der Signalaufspaltung: $\mathrm{s}=$ Singulett, br s $=$ breites Singulett, $\mathrm{d}$ $=$ Dublett, $\mathrm{t}=$ Triplett, $\mathrm{q}=$ Quartett, $\mathrm{m}=$ Multiplett, $\mathrm{dd}=$ doppeltes Dublett, $\mathrm{dt}=$ doppeltes Triplett; Spektren wurden unter der Annahme einer Kopplungsaufspaltung 1. Ordnung ausgewertet. Sämtliche Kopplungskonstanten sind als Betrag angegeben. Abkürzungen für Zuordnungen der Signale: Ar-H = arylisch. $-{ }^{13}$ C-NMR: Bruker AM 250 (62.9 MHz), $\delta=$ 77.0 für Deuterochloroform, 128.0 für $\mathrm{C}_{6} \mathrm{D}_{5} \mathrm{H}$. Die Multiplizität der Signale wurde durch die DEPT-Aufnahmetechnik $($ DEPT $=$ Distortionless Enhancement by Polarisation Transfer; Pulswinkel des letzten Impulses $135^{\circ}$ ) bestimmt und wie folgt angegeben: DEPT: + = primäre oder tertiäre (positives DEPT-Signal), - = sekundäre (negatives DEPT-Signal), $\mathrm{C}_{\text {quart }}=$ quartäre C-Atome (DEPT-Signalintensität Null). Abkürzung für die Zuordnung der Signale: Ar-C = arylisch. - IR: Bruker IFS 66. - MS: Varian MAT CH 7, MAT 731. Säulenchromatographie (SC): Chromatographische Trennungen erfolgten an Merck Kieselgel 60 (0.063-0.200 mm, 70-230 mesh ASTM) oder an basischem Aluminiumoxid der Firma ICN (ICN Alumina Activity I, 50-200 $\mu \mathrm{m}$ ). Die Säulendimensionen werden als „Säulendurchmesser $\times$ Höhe der Säule“ angegeben. Alle Laufmittel wurden nur destilliert verwendet. Der Petrolether besaß einen Siedepunkt von $30-50^{\circ} \mathrm{C}$. Dünnschichtchromatographie (DC): Machery-Nagel, Fertigfolien Alugram ${ }^{\circledR}$ Sil G/UV 254 . Detektion unter UV-Licht bei 254 nm, Entwicklung mit Molypdatophosphorsäure- (10\%-ige Lsg. in Ethanol) Kaliumpermanganat (1 g Kaliumpermanganat, $2 \mathrm{~g}$ Essigsäure, $5 \mathrm{~g}$ Natriumhydrogencarbonat in $100 \mathrm{ml}$ Wasser) oder Ninhydrin-Tauchreagenz (0.3 g Ninhydrin und $3 \mathrm{~g}$ Eisessig in $100 \mathrm{ml} n$ Butanol). - Schmelzpunkte: Schmelzpunktapparatur nach Dr. Totolli der Fa. Büchi; die gemessenen Werte sind unkorrigiert. - Elementaranalysen: Mikroanalytisches Laboratorium des Institutes für Organische Chemie der Universität 
Göttingen. - Umsetzungen ohne Temperaturangabe wurden bei $20^{\circ} \mathrm{C}$ durchgeführt. Lösungsmittel wurden nach üblichen Laboratoriumsmethoden getrocknet und unter Stickstoff oder Argon destilliert. - Sämtliche nicht-wäßrigen Reaktionen wurden unter Argon- oder Stickstoffatmosphäre in ofengetrockneten, geflammten Kolben durchgeführt. - Nicht gesondert aufgeführte Chemikalien sind kommerziell erhältlich.

\section{Darstellung der Verbindungen}

\subsection{Allgemeine Arbeitsvorschriften}

Allgemeine Arbeitsvorschrift (AAV 1) für die Herstellung von Tosylhydrazonen aus Aldehyden: Tosylhydrazin wird in $100 \mathrm{ml}$ Methanol suspendiert und $10 \mathrm{~min}$ bei $20^{\circ} \mathrm{C}$ gerührt. $\mathrm{Zu}$ der Suspension werden 1.15 äq in Methanol gelöster Aldehyd gegeben und weitere $10 \mathrm{~min}$ bei $20^{\circ} \mathrm{C}$ gerührt. Nach 1 min löst sich das Tosylhydrazin auf und es entsteht eine gelbliche Lösung, aus der nach weiteren 2 min ein weisser Feststoff ausfällt. Es wird 30 min unter Rückfluss erwärmt, das Zielprodukt aus der abgekühlten Lösung abfiltriert und der Filterrückstand mit $30 \mathrm{ml}$ eiskaltem Methanol gewaschen. Der Feststoff wird $1 \mathrm{~d}$ bei $20^{\circ} \mathrm{C}$ und $10^{-2}$ Torr von flüchtigen Substanzen befreit. Das so hergestellte Tosylhydrazon weist keine fremden Signale auf.

\section{Allgemeine Arbeitsvorschrift (AAV 2) für die Herstellung von Aryldiazomethanderivaten aus Tosylhydrazonen: Es werden 2.0 eq. Natrium in $15 \mathrm{ml}$} (16.7 g) Ethylenglycol in einer $100 \mathrm{ml}$ Pyrexflasche aufgelöst und das Tosylhydrazon (1 eq.) eingerührt. Der zähe Brei wird mit $50 \mathrm{ml}$ Hexan überschichtet, die Flasche gut verschlossen und die Reaktionsmischung unter Rühren für 5 min auf $90{ }^{\circ} \mathrm{C}$ erwärmt. Das Reaktionsgefäß wird in einem Eisbad schnell abgekühlt, die rote Hexanlösung abpipettiert und in einen mit Aluminiumfolie umwickelten eisgekühlten Kolben überführt. Dieser Vorgang wird so oft wiederholt, bis sich keine Diazokomponente mehr in der Hexanphase löst ( $5 \times$, Rotfärbung). Das Hexan wird unter vermindertem Druck bei $20^{\circ} \mathrm{C}$ entfernt und die 
Aryldiazomethanderivate können in für weitere Synthesen ausreichender Reinheit als roter Feststoff isoliert werden.

Allgemeine Arbeitsvorschrift (AAV 3) für die Herstellung von Cinnamyldiazoacetatderivaten aus Cinnamylacetoacetat und Tosylazid: $\mathrm{Zu}$ einer Lösung von $50 \mathrm{mmol}$ Tosylazid und $50 \mathrm{mmol}$ Acetoacetat in $200 \mathrm{ml}$ Dichlormethan werden $1.2 \mathrm{mmol}$ Tetrabutylammoniumbromid zugegeben und $30 \mathrm{~min}$ gerührt. Es werden $10 \mathrm{ml} 10 \mathrm{~N} \mathrm{NaOH}-$ Lösung zugegeben und $12 \mathrm{~h}$ bei $20^{\circ} \mathrm{C}$ gerührt. Die Reaktionsmischung wird dann mit

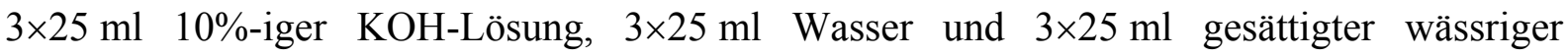
Kochsalzlösung gewaschen und über $\mathrm{NaSO}_{4}$ getrocknet. Das Rohprodukt kann unter geringem Ausbeuteverlust chromatographisch (5-fache Menge Silica, Hexan/ $\mathrm{CH}_{2} \mathrm{Cl}_{2}$ 1:1) gereinigt werden.

\section{Allgemeine Arbeitsvorschrift (AAV 4) für Kreuzkupplung unter} $\mathbf{P d C l}_{2}$ (dppf)-Katalyse: ${ }^{[5]}$ Es wird eine Lösung von Cyclopent-3-en-1-ylmagnesiumbromid (4) aus Cyclopenten-4-ylbromid (81) (45 mmol) und Magnesium (50 mmol) in trockenem $\mathrm{Et}_{2} \mathrm{O}(30 \mathrm{ml})$ hergestellt ${ }^{[6]}$ und mit einer Kannüle zu einer Mischung aus Arylhalogenid (18 mmol) und dem Katalysator $(3 \mathrm{~mol} \%)$ in wasserfreiem $\mathrm{Et}_{2} \mathrm{O}(100 \mathrm{ml})$ bei Raumtemperatur unter Schutzgas schnell zugegeben. Während der Kupplungsreaktion tritt nach 10 min Rühren eine schnelle Farbänderung von orange über gelb nach grün oder braun auf. Im Falle der Aryljodide ist die Reaktion etwas exotherm und die zu Anfang tiefgrüne Farbe geht über hellgrün oder gelb nach braun über. Die Reaktionsmischung wird für weitere $12 \mathrm{~h}$ bei $20{ }^{\circ} \mathrm{C}$ gerührt und dann in eine eiskalte gesättigte $\mathrm{NH}_{4} \mathrm{Cl}$-Lösung $(100 \mathrm{ml})$ gegossen. Es werden $100 \mathrm{ml} \mathrm{Et} \mathrm{f}_{2} \mathrm{O}(100 \mathrm{ml})$ zugegeben und die Phasen getrennt. Die organische Phase wird mit jeweils 50 ml 10\%-iger $\mathrm{NH}_{4} \mathrm{Cl}$-Lösung und gesättigter wässriger Kochsalzlösung gewaschen, getrocknet und das Lösungsmittel entfernt. Der Rückstand wird säulenchromatographisch gereinigt.

\section{Allgemeine Arbeitsvorschift für die rhodiumoctanoatkatalysierte Addition von tertButyldiazoacetat an Alkene (AAV 5): Zu einer gerührten Lösung des jeweiligen Alkens (25 mmol) und Dirhodiumtetraoctanoat $(0.5 \mathrm{~mol} \%)$ in $100 \mathrm{ml}$ trockenem Dichlormethan wurde tertButyl diazoacetat $(37.5 \mathrm{mmol})$ bei $0-+5{ }^{\circ} \mathrm{C}$ während $12 \mathrm{~h}$ zugetropft. Nach Entfernen des Lösungsmittels wird das Reaktionsprodukt säulenchromatographisch gereinigt.}


Bei schlechter Löslichkeit im Laufmittel wird das Rohprodukt vor der Chromatographie aus Dichlormethan an $20 \mathrm{~g}$ Kieselgel adsorbiert.

Allgemeine Arbeitsvorschrift zur Reduktion von tertButylestern 162 (AAV 6): Zu einer Lösung des jeweiligen tertButylesters $162(10 \mathrm{mmol})$ in wasserfreiem Diethylether $(80 \mathrm{ml})$ wurden unter Rühren 7.5 mmol $\mathrm{LiAlH}_{4}\left(6.4 \mathrm{ml}\right.$ einer $1.17 \mathrm{M}$ Lösung in $\left.\mathrm{Et}_{2} \mathrm{O}\right)$ während 20 min bei $20{ }^{\circ} \mathrm{C}$ zugetropft. Danach wurde die Reaktionsmischung bei $34{ }^{\circ} \mathrm{C} 1 \mathrm{~h}$ gerührt, auf 10 ${ }^{\circ} \mathrm{C}$ abgekühlt, mit gesättigter $\mathrm{Na}_{2} \mathrm{SO}_{4}$-Lösung gequencht, getrocknet und das Lösungsmittel unter vermindertem Druck entfernt. Die erhaltenen Alkohole 163 sind für weitere synthetische Anwendungen ausreichend rein.

Allgemeine Arbeitsvorschrift zur Synthese von Jodiden aus Alkoholen (AAV 7): ${ }^{[102]} \mathrm{Zu}$ einer Lösung des jeweiligen Alkohols 163 (4 mmol), Imidazol $(5.5 \mathrm{mmol})$ und $\mathrm{Ph}_{3} \mathrm{P}(5.2$ $\mathrm{mmol}$ ) in einer Mischung aus wasserfreiem $\mathrm{MeCN}(30 \mathrm{ml})$ und trockenem $\mathrm{Et}_{2} \mathrm{O}$ [50 ml; bei schlechter Löslichkeit der Alkohole 163a,d wurde wasserfreies THF (20 ml) zugesetzt] wurde unter Rühren unter Lichtschutz und Argon Jod $(6.0 \mathrm{mmol})$ bei $-10{ }^{\circ} \mathrm{C}$ in einer Portion zugegeben. Nach weiteren 30 min Rühren bei $0{ }^{\circ} \mathrm{C}$ wurde der Reaktionsmischung $\mathrm{Et}_{2} \mathrm{O}$ (100 ml) zugesetzt und die Lösung wurde nacheinander mit gesättigter wässriger $\mathrm{Na}_{2} \mathrm{~S}_{2} \mathrm{O}_{3}$-Lösung $(50 \mathrm{ml})$ und gesättigter wässriger Kochsalzlösung $(100 \mathrm{ml})$ gewaschen, getrocknet und das Lösungsmittel unter vermindertem Druck unter Lichtschutz entfernt. Der Rückstand wurde in möglichst wenig $\mathrm{CH}_{2} \mathrm{Cl}_{2}(5-35 \mathrm{ml})$ aufgenommen und säulenchromatographisch gereinigt.

Allgemeine Arbeitsvorschrift für die Kreuzkupplung von Jodiden mit Alkylmagnesiumbromiden unter Katalyse von $\mathrm{Li}_{2} \mathbf{C u C l}_{4}$ (AAV 8): ${ }^{[7]} \mathrm{Zu}$ einer Lösung von Alkylmagnesiumbromid (1.5-2 eq.) in trockenem $\mathrm{Et}_{2} \mathrm{O}$ wirde das jeweilige Jodid 9 (5 mmol) in wasserfreiem THF $(50 \mathrm{ml})$ bei $-5{ }^{\circ} \mathrm{C}$ zugegeben. Die Reaktionsmischung wird 5 min gerührt und dann eine Lösung von aus $\mathrm{LiCl}(0.5 \mathrm{mmol})$ und wasserfreiem $\mathrm{CuCl}_{2}(0.25 \mathrm{mmol})$ frisch zubereitetem $\mathrm{Li}_{2} \mathrm{CuCl}_{4}(5 \mathrm{~mol} \%)$ in THF (3 ml) zugegeben. Nach Abklingen der heftigen Reaktion wird die Reaktionsmischung $0.5 \mathrm{~h}$ bei $0{ }^{\circ} \mathrm{C}$ gerührt und dann in eine eiskalte Mischung aus gesättigter $\mathrm{NH}_{4} \mathrm{Cl}$-Lösung $(50 \mathrm{ml})$ und $\mathrm{Et}_{2} \mathrm{O}(100 \mathrm{ml})$ geschüttet. Die organische Phase wurde mit gesättigter wässriger $\mathrm{NH}_{4} \mathrm{Cl}$-Lösung $(50 \mathrm{ml})$ und gesättigter wässriger Kochsalzlösung $(50 \mathrm{ml})$ gewaschen, getrocknet und das Lösungsmittel unter vermindetem Druck entfernt. Der Rückstand wird säulenchromatographisch aufgearbeitet. 


\subsection{Beschreibung der Synthesen}

cis-1,4-Dichloro-2-buten (27): Unter Eiskühlung wurden zu einer Lösung von $108 \mathrm{~g}(1.00 \mathrm{Mol})$ trockenem cis-2-Buten-1,4-diol in $200 \mathrm{ml}$

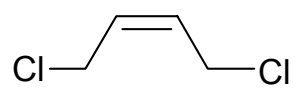
trockenem Pyridin (204 g, 2.59 Mol, 2.59 äq) $250 \mathrm{ml} \mathrm{(408} \mathrm{g,} 3.43 \mathrm{Mol}, 3.34$ äq) $\mathrm{SOCl}_{2}$ so zugetropft, dass die Innentemperatur der Reaktionsmischung $10{ }^{\circ} \mathrm{C}$ nicht überschritt. Es wurden $2 \mathrm{~h}$ gerührt, und das braune Öl weitere $24 \mathrm{~h}$ ohne Rühren bei $20^{\circ} \mathrm{C}$ stehen gelassen. Die Rohsubstanz wurde dann in einen Scheidetrichter mit $1.5 \mathrm{~kg}$ Eis gegossen, die Phasen getrennt, die organische Phase mit $500 \mathrm{ml}$ gesättigter $\mathrm{NaHCO}_{3}$-Lösung gewaschen und mit $500 \mathrm{ml}$ gesättigter wässriger Kochsalzlösung ausgeschüttelt. Nach Trocknen über $\mathrm{CaCl}_{2}$ wurde das braune Öl über eine $30 \mathrm{~cm}$ Vigreuxkolonne destilliert. Man erhielt $94.1 \mathrm{~g}$ farbloses Zielprodukt (27) mit einem Siedepunkt von $152{ }^{\circ} \mathrm{C}$ (758 Torr). Die physikalischen Eigenschaften der Substanz stimmen mit den Literaturangaben überein.

${ }^{1} \mathrm{H}-\mathrm{NMR}\left(250 \mathrm{MHz}, \mathrm{CDCl}_{3}\right): \delta=4.09\left(\mathrm{~m}, \quad 4 \mathrm{H}, \mathrm{CH}_{2}\right), 5.82(\mathrm{~m}, 2 \mathrm{H}, \mathrm{CH})-{ }^{13} \mathrm{C}-\mathrm{NMR}$ (62.9 MHz, $\left.\mathrm{CDCl}_{3}, \mathrm{DEPT}\right): \delta=124.3(-)\left(\mathrm{CH}_{2}\right), 41.4(+)(\mathrm{CH})$.

Cyclopent-3-encarbonsäure (31): In einem 1-1 Dreihalskolben mit Rückflusskühler wurden unter kräftigem Rühren zu einer Lösung von $33.0 \mathrm{~g}$ (250 mmol) Dimethylmalonat und $50 \mathrm{ml}$ trockenem DMPU in $450 \mathrm{ml}$ trockenem THF bei $0{ }^{\circ} \mathrm{C} \mathrm{CO}_{2} \mathrm{H}$ $5.00 \mathrm{~g}$ (629 mmol, 2.5 eq.) Lithiumhydrid in einer Portion zugegeben. Nach beendeter Wasserstoffentwicklung (2 h) wurden $28.4 \mathrm{ml}$ (269 mmol, 1.1 eq.) cis-1,4-Dichlor-2-buten schnell zugegeben. Die Reaktionsmischung wurde für $24 \mathrm{~h}$ auf $40-45{ }^{\circ} \mathrm{C}$ erwärmt. Danach wurden $31.50 \mathrm{~g}$ (750 mmol, 3.0 eq.) festes Lithiumhydroxid Monohydrat zugegeben. Nach 24 h Rühren wurden $350 \mathrm{ml}$ Wasser zugegeben, 10 min gerührt, und Neutralstoffe durch 3maliges Ausschütteln mit je $500 \mathrm{ml}$ Ethylacetat entfernt. Die wässrige Lösung wurde mit $160 \mathrm{ml} 6 \mathrm{~N}$ Salzsäure angesäurert, und mit 3×500 ml Ethylacetat ausgeschüttelt. Die organische Phase wurde mit gesättigter wässriger Kochsalzlösung gewaschen, mit $\mathrm{MgSO}_{4}$ getrocknet und das Lösungsmittel im Vakuum entfernt. Die 3-Cyclopenten-1,1-Dicarbonsäure wurde ohne weitere Reinigung auf 170 - $175{ }^{\circ} \mathrm{C}$ erwärmt, bis die $\mathrm{CO}_{2}$-Entwickelung beendet 
war (ca. 2 h). Die erhaltene Flüssigkeit wurde ohne Fraktionstrennung destilliert. Es wurden $23.0 \mathrm{~g}$ ( $205 \mathrm{mmol}, 82 \%$ ) der Zielverbindung als farbloses Öl mit einem Siedepunkt von $88^{\circ} \mathrm{C}(2 \mathrm{~mm})$ isoliert.

- IR (KBr): $v\left[\mathrm{~cm}^{-1}\right]=3060,2859,2557,1702,1618,1414,1300,1223,1179,949,708$. ${ }^{1} \mathrm{H}-\mathrm{NMR}\left(250 \mathrm{MHz}, \mathrm{CDCl}_{3}\right): \delta=2.70\left(\mathrm{~d},{ }^{3} \mathrm{~J}=8.0 \mathrm{~Hz}, 4 \mathrm{H}, \mathrm{CH}_{2}\right), 3.18$ (quin, ${ }^{3} J=8.0 \mathrm{~Hz}$, $1 \mathrm{H}, \mathrm{CH}), 5.70(\mathrm{~s}, 2 \mathrm{H}, \mathrm{CH}), 11.6-12.1$ (bs, $\left.1 \mathrm{H}, \mathrm{CO}_{2} \mathrm{H}\right) .-{ }^{13} \mathrm{C}-\mathrm{NMR}\left(62.9 \mathrm{MHz}, \mathrm{CDCl}_{3}\right.$, DEPT): $\delta=36.19(-)\left(\mathrm{CH}_{2}\right), 41.35(+)(\mathrm{CH}), 128.90(+)(\mathrm{CH}), 182.93\left(\mathrm{C}_{\text {quart }}, \mathrm{CO}_{2} \mathrm{H}\right)$. Die physikalischen Eigenschaften stimmen mit den Literaturangaben überein.

Cyclopent-3-encarbonsäure (31): Es wurden in einem 11 Dreihalskolben mit KPG-Rührer unter Stickstoff $33.0 \mathrm{~g} \quad(250 \mathrm{mmol})$ Dimethylmalonat, $50 \mathrm{ml}$ trockenes DMPU (N,N'-Dimethylpropylharnstoff) und $450 \mathrm{ml}$ trockenes THF

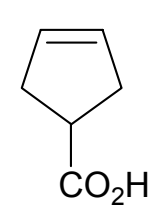
vorgelegt und mit einem Eisbad gekühlt. Zu der Lösung wurden $5.00 \mathrm{~g}(629 \mathrm{mmol}) \mathrm{LiH}$ in einer Portion zugegeben. Die Kühlung wurde entfernt und der Schutzgasfluss gestoppt. Es wurde so lange gerührt, bis die Wasserstoffentwicklung beendet war $(2-5 \mathrm{~h})$. Dann wurden $28.4 \mathrm{ml}$ (269 mmol) cis-1,4-Dichlorbut-2-en mit einer Spritze schnell zugegeben. Die Reaktionsmischung wurde $24 \mathrm{~h}$ auf $45^{\circ} \mathrm{C}$ erwärmt, abgekühlt auf $20^{\circ} \mathrm{C}$, und nacheinander vorsichtig $50 \mathrm{ml}$ Wasser und $31.5 \mathrm{~g}(750 \mathrm{mmol})$ festes Lithiumhydroxid Monohydrat zugegeben. Nach weiteren $24 \mathrm{~h}$ Rühren bei Raumtemperatur wurden $350 \mathrm{ml}$ Wasser zugegeben, weitere 10 min gerührt und die Reaktionsmischung in einen 21 Scheidetrichter überführt. Neutralstoffe wurden durch Ausschütteln mit $5 \times 500 \mathrm{ml}$ Ethylacetat entfernt. Jede der $500 \mathrm{ml}$ Ethylacetatfraktionen wurde mit $30 \mathrm{ml}$ gesättigter wässriger Kochsalzlösung zurückextrahiert. Die vereinigten wässrigen Phasen wurden mit $160 \mathrm{ml} 6 \mathrm{~N} \mathrm{HCl}$ angesäuert und mit $3 \times 500 \mathrm{ml}$ Ethylacetat extrahiert. Die vereinigten organischen Phasen wurden nachfolgend mit $3 \times 100 \mathrm{ml} 3 \mathrm{~N} \mathrm{HCl}$ und $2 \times 50 \mathrm{ml}$ gesättigter wässriger Kochsalzlösung gewaschen, mit Natriumsulfat getrocknet und das Lösungsmittel unter vermindertem Druck entfernt. Letzte Spuren Lösungsmittel können in $1 \mathrm{~h}$ bei 0.1 Torr und $20^{\circ} \mathrm{C}$ entfernt werden. Es können 35.8 g Cyclopent-3-en-1,1-dicarbonsäure isoliert werden, die durch nachfolgendes 2-stündiges Erwärmen auf $175^{\circ} \mathrm{C}$ ohne Lösungsmittel zur Cyclopent-3-encarbonsäure decarboxyliert. Es können nach Destillation (Sdp $\left.88^{\circ} \mathrm{C}, \quad 2 \mathrm{Torr}\right) \quad 23.0 \mathrm{~g} \quad(89 \%$; Gesamtausbeute $82 \%)$ der Titelverbindung isoliert werden. - IR $(\mathrm{KBr}): v\left[\mathrm{~cm}^{-1}\right]=2859(\mathrm{~b})$, 1702, 1414, 1300, 1223, 1179, 949, 708. - ${ }^{1} \mathrm{H}-\mathrm{NMR}\left(250 \mathrm{MHz}, \mathrm{CDCl}_{3}\right): \delta=1.60\left(\mathrm{~d},{ }^{3} \mathrm{~J}=\right.$ 
$8.0 \mathrm{~Hz}, 4 \mathrm{H}, \mathrm{CH}_{2}$ ), 3.10 (quin, ${ }^{3} \mathrm{~J}=8.0 \mathrm{~Hz}, 1 \mathrm{H}, \mathrm{CH}$ ), 5.70 (s, $2 \mathrm{H}, \mathrm{CH}$ ), 11.2-11.8 (bs, $1 \mathrm{H}$, $\mathrm{OH}) .-{ }^{13} \mathrm{C}-\mathrm{NMR}\left(62.9 \mathrm{MHz}, \mathrm{CDCl}_{3}, \mathrm{DEPT}\right): \delta=35.1(-) \mathrm{CH}_{2}, 43.3(+) \mathrm{CH}, 128.5(+) \mathrm{CH}$, 183.5 $\mathrm{CO}_{2} \mathrm{H}$. Die physikalischen Eigenschaften stimmen mit den Literaturangaben überein.

Cyclopenten-4-carbonsäure-tertbutylester (32): $\quad \mathrm{Zu} \quad 11.8 \mathrm{~g} \quad$ Cyclopent3encarbonsäure wurden unter Stickstoffkühlung $150 \mathrm{ml}$ Isobuten in eine Ampulle kondensiert und $3 \mathrm{ml} \mathrm{H}_{2} \mathrm{SO}_{4}$ zugegeben. Die Reaktionsmischung wurde bei $20{ }^{\circ} \mathrm{C} \mathrm{CO}_{2}{ }^{t} \mathrm{Bu}$ 3 Tage gerührt, mit $100 \mathrm{ml}$ gesättigter $\mathrm{NaHCO}_{3}$-Lösung geschüttelt, die wässrige Phase mit $2 \times 100 \mathrm{ml} \mathrm{CH} \mathrm{Cl}_{2}$ ausgeschüttelt, und die organische Phase mit $\mathrm{Na}_{2} \mathrm{SO}_{4}$ getrocknet. Das Natriumsulfat wurde abfiltriert, das Lösungsmittel im Vakuum entfernt und $17.9 \mathrm{~g}$ der Zielverbindung isoliert. - IR (KBr): $v\left[\mathrm{~cm}^{-1}\right]=3422,3058,2849,1742(\mathrm{C}=\mathrm{O}), 1618$ $(\mathrm{HC}=\mathrm{CH}), 1480,1392,1368,1297,1136,849,680 .-{ }^{1} \mathrm{H}-\mathrm{NMR}\left(250 \mathrm{MHz}, \mathrm{CDCl}_{3}\right): \delta=1.44$ $\left(\mathrm{s}, 9 \mathrm{H}, \mathrm{C}\left(\mathrm{CH}_{3}\right)_{3}\right), 2.59\left(\mathrm{~d},{ }^{3} J=8.0 \mathrm{~Hz}, 4 \mathrm{H}, \mathrm{CH}_{2}\right.$ ), 3.01 (quin, ${ }^{3} \mathrm{~J}=8.0 \mathrm{~Hz}, 1 \mathrm{H}, \mathrm{CH}$ ), 5.63 (s, $2 \mathrm{H}, \mathrm{CH}) .-{ }^{13} \mathrm{C}-\mathrm{NMR}\left(62.9 \mathrm{MHz}, \mathrm{CDCl}_{3}, \mathrm{DEPT}\right): \delta=27.07(+) \mathrm{CH}_{3}, 36.28(-) \mathrm{CH}_{2}, 42.49$ (+) $\mathrm{CH}, 79.96 \mathrm{C}_{\text {quart }}, 128.96(+) \mathrm{CH}$. - MS (70 eV, EI), m/z (\%):168 (5) $\left[\mathrm{M}^{+}\right], 112(35)\left[\mathrm{M}^{+}\right.$ $\left.-\mathrm{C}_{4} \mathrm{H}_{8}\right], 67(58)\left[\mathrm{C}_{5} \mathrm{H}_{7}^{+}\right], 57(100)\left[\mathrm{C}_{4} \mathrm{H}_{9}\right]$.

exo,exo-Bicyclo[3.1.0] hexan-3,6-dicarbonsäure-3-tbutylester-6-

ethylester (33a): $2.637 \mathrm{~g}(15.7 \mathrm{mmol}) \quad$ Cyclopenten-4-carbonsäure- ${ }^{t}$ butylester und $17.6 \mathrm{mg} \mathrm{Rh}_{2}(\mathrm{OAc})_{4}$ (3.98 mmol, $0.25 \mathrm{~mol} \%$ ) wurden in $15 \mathrm{ml} \mathrm{CH}_{2} \mathrm{Cl}_{2}$ vorgelegt, und mit einer Spritzenpumpe $1.789 \mathrm{~g} \quad(15.7 \mathrm{mmol}, 1$ äq) Ethyldiazoacetat in $15 \mathrm{ml} \mathrm{CH}_{2} \mathrm{Cl}_{2}$ während $12 \mathrm{~h}$ bei $20^{\circ} \mathrm{C}$ unter Rühren<smiles>CCOC(=O)C1C2CC3C(C(=O)OCC)C2C31</smiles>
zugetropft. Der Katalysator wurde durch Filtration der Reaktionsmischung über basisches Aluminiumoxid (Säule $5 \times 3 \mathrm{~cm}$, Laufmittel $\mathrm{CH}_{2} \mathrm{Cl}_{2}$ ) entfernt und das Lösungsmittel im Vakuum abdestilliert. Es entstanden 2 Diastereomere im Verhältnis 7:3. Es konnten nach säulenchromatographischer Reinigung (170 g Silica, Säule $27 \times 4 \mathrm{~cm}$, Laufmittel Petrolether:Diethylether 9:1, $\left.\mathrm{R}_{f}=0.30\right) 2.52 \mathrm{~g}$ (9.91 mmol, 63\%) Zielprodukt isoliert werden. - IR $(\mathrm{KBr}): v\left[\mathrm{~cm}^{-1}\right]=? ? ?,-{ }^{1} \mathrm{H}-\mathrm{NMR}\left(250 \mathrm{MHz}, \mathrm{CDCl}_{3}\right): \delta=1.20\left(\mathrm{~s}, 9 \mathrm{H},\left(\mathrm{CH}_{3}\right)_{3}\right), 1.40(\mathrm{t}$, $\left.{ }^{3} J=7.0 \mathrm{~Hz}, 3 \mathrm{H}\right), 1.80\left(\mathrm{~d},{ }^{3} J=4.0 \mathrm{~Hz} 2 \mathrm{H},(\mathrm{CH})_{2}\right), 2.20\left(\mathrm{~d},{ }^{3} J=4.0 \mathrm{~Hz}, 4 \mathrm{H},\left(\mathrm{CH}_{2}\right)_{2}\right), 2.83$ (quin, $\left.{ }^{3} J=4.0 \mathrm{~Hz} 1 \mathrm{H}, \mathrm{CH}\right), 4.15\left(\mathrm{q},{ }^{3} J=7.0 \mathrm{~Hz} 2 \mathrm{H}, \mathrm{OCH}_{2}\right) .-{ }^{13} \mathrm{C}-\mathrm{NMR}\left(62.9 \mathrm{MHz}, \mathrm{CDCl}_{3}\right.$, DEPT): $\delta=14.3(+) \mathrm{CH}_{3}, 23.6(+) \mathrm{CH}, 25.7(+) \mathrm{CH}_{3}, 27.0(+) \mathrm{CH}, 29.7(-) \mathrm{CH}_{2}, 44.1(+)$ $\mathrm{CH}, 60.1(-) \mathrm{CH}_{2}, 79.9 \mathrm{C}_{\text {quart }}, 171.7 \mathrm{C}=\mathrm{O}, 175.3 \mathrm{C}=\mathrm{O}$. 
exo,exo-Bicyclo[3.1.0] hexan-3,6-dicarbonsäure (34a): Es wurden $1.50 \mathrm{~g}$ (5.90 mmol) Bicyclo[3.1.0]hexan-3,6-dicarbonsäure-3-tertbutylester-6ethylester 33a in $25 \mathrm{ml}$ einer Mischung aus $50 \% \mathrm{MeOH}$ und $50 \%$ THF gelöst. Es wurden $1.47 \mathrm{~g}$ (35 mmol, äq) Lithiumhydroxyd Monohydrat in $6 \mathrm{ml}$ Wasser hinzugegeben und $24 \mathrm{~h}$ bei $20^{\circ} \mathrm{C}$ gerührt. Die Reaktionsmischung wurde mit $1 \mathrm{M} \mathrm{H} \mathrm{H}_{3} \mathrm{PO}_{4}$ auf $\mathrm{pH} 3$ gebracht, mit $4 \times 150 \mathrm{ml}$ Ethylacetat

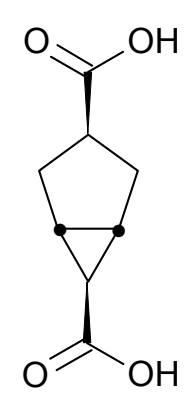
ausgeschüttelt, und die organische Phase über $\mathrm{Na}_{2} \mathrm{SO}_{4}$ getrocknet. Es wurden $1.00 \mathrm{~g}$ (5.90 mmol, 100\%) der Zielverbindung isoliert, die ohne weitere Charakterisierung zur Estersynthese verwendet wurde.

exo,exo-Bicyclo[3.1.0]hexan-3,6-dicarbonsäure-3, 6-di(4-npentylphenyl)ester (35a): Es wurden $368 \mathrm{mg}$ (2.16 mmol) exo,exo-Bicyclo[3.1.0]hexan-3,6dicarbonsäure, $710 \mathrm{mg}$ (4.33 mmol, 2 äq) 4-nPentylphenol und $43 \mathrm{mg}$ (0.35 mmol, $8 \mathrm{Mol} \%$ ) DMAP in $2 \mathrm{ml} \mathrm{DMF} \mathrm{vorgelegt} \mathrm{und} \mathrm{auf} 0{ }^{\circ} \mathrm{C}$ gekühlt. Dann wurden $1.037 \mathrm{~g}$ (4.76 mmol, 2.2 äq) DCC zugegeben und bei $20{ }^{\circ} \mathrm{C}$ weitere $18 \mathrm{~h}$ gerührt. Die Reaktionsmischung wurde dann in $50 \mathrm{ml}$ THF aufgenommen und säulenchromatographisch (Säule $25 \times 3 \mathrm{~cm}$, Laufmittel $\mathrm{PE}: \mathrm{Et}_{2} \mathrm{O}$ 3:1) gereinigt. Es wurden $421 \mathrm{mg}$ (0.911 mmol, 42\%) Zielprodukt erhalten. $-{ }^{1} \mathrm{H}-\mathrm{NMR}\left(250 \mathrm{MHz}, \mathrm{CDCl}_{3}\right): \delta=0.90\left(\mathrm{t},{ }^{3} J=6.5 \mathrm{~Hz}, 6 \mathrm{H}, 2 \times\right.$ $\left.\mathrm{CH}_{3}\right), 1.29-1.35\left(\mathrm{~m}, 8 \mathrm{H}, 4 \times \mathrm{CH}_{2}\right), 1.45(\mathrm{~m}, 1 \mathrm{H}, 6-\mathrm{H}), 1.55-1.67(\mathrm{~m}, 4 \mathrm{H}$, $\left.2 \times \mathrm{CH}_{2}\right), 1.69-1.72(\mathrm{~m}, 2 \mathrm{H}, 1-\mathrm{H}, 5-\mathrm{H}), 2.25-2.45(\mathrm{~m}, 4 \mathrm{H}, 2-\mathrm{H}, 4-\mathrm{H}), 2.60$ (t, $\left.{ }^{3} J=8.0 \mathrm{~Hz}, 4 \mathrm{H}, \mathrm{Ar}-\mathrm{CH}_{2}\right), 2.68-2.79$ (m, $\left.1 \mathrm{H}, 3-\mathrm{H}\right), 6.95-7.05$ (m, $4 \mathrm{H}$, Ar-H), $7.14-7.23$ (m, $4 \mathrm{H}, \mathrm{Ar}-\mathrm{H}) .-{ }^{13} \mathrm{C}-\mathrm{NMR}\left(62.9 \mathrm{MHz}, \mathrm{CDCl}_{3}\right.$, DEPT):

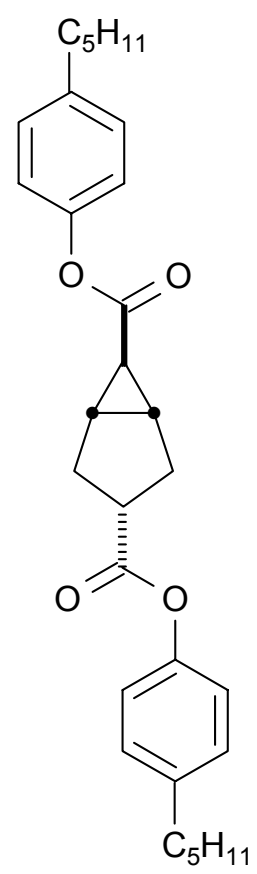
$\delta=14.0(+) 2 \times \mathrm{CH}_{3}, 22.2(+) \mathrm{C}-3,22.5(-) 2 \times \mathrm{CH}_{2}, 28.5(+) \mathrm{C}-1, \mathrm{C}-5,31.1$ (-) $2 \times \mathrm{CH}_{2}, 31.42 \times \mathrm{CH}_{2}, 35.5$ (-) $2 \times \mathrm{CH} 2,39.5$ (+) C-6, 120.98 (-) Ar-C, 121.15 (-) Ar-C, 129.22 (-) Ar-C, 129.27 (-) Ar-C, 140.38 Ar-C quart, 140.55 Ar- $\mathrm{C}_{\text {quart, }}$ 148.49 $\mathrm{Ar}-\mathrm{C}_{\text {quart, }}$ 148.54 $\mathrm{Ar}-\mathrm{C}_{\text {quart }}, 171.96 \mathrm{C}=\mathrm{O}, 173.30$ $\mathrm{C}=\mathrm{O}$. $-\mathrm{MS}(70 \mathrm{eV}, \mathrm{EI}), m / z \quad(\%): 462(22)\left[\mathrm{M}^{+}\right], 299(100)\left[\mathrm{M}^{+}-\right.$ $\mathrm{C}_{11} \mathrm{H}_{15} \mathrm{O}$ ], 164 (25), 107 (40). $\mathrm{C}_{30} \mathrm{H}_{38} \mathrm{O}_{4}$ (462.63): ber. C 78.89, H 8.28\%; gem. C 78.15, H 7.92\%.

exo,exo-Bicyclo[3.1.0]hexan-3,6-dicarbonsäure-3,6di(4-npropyloxyphenyl)ester (35b): $\quad$ Es $\quad$ wurden $300 \mathrm{mg} \quad(1.76 \mathrm{mmol})$ 
exo,exo-Bicyclo[3.1.0]hexan-3,6-dicarbonsäure, $\quad 536 \mathrm{mg} \quad(3.53 \mathrm{mmol}, \quad 2$ äq) 4- $n$ Propyloxyphenol und $43 \mathrm{mg}$ (0.35 mmol, $10 \mathrm{Mol} \%)$ DMAP in $2 \mathrm{ml}$ DMF vorgelegt und auf $0{ }^{\circ} \mathrm{C}$ gekühlt. Dann wurden $846 \mathrm{mg}$ (3.88 mmol, 2.2 äq) DCC zugegeben und bei $20^{\circ} \mathrm{C}$ weitere $18 \mathrm{~h}$ gerührt. Die Reaktionsmischung wurde dann in $50 \mathrm{ml}$ THF aufgenommen und säulenchromatographisch (180 g Kieselgel, Säule 25×3 cm, Laufmittel PE:Et $2 \mathrm{O}$ 3:1) gereinigt. Es wurden $356 \mathrm{mg}$ (0.812 mmol, 46\%) Zielprodukt erhalten. . - IR (KBr): v[cm $\left.{ }^{1}\right]=3110,3067,3046,2968,2940,2880,1743(\mathrm{C}=\mathrm{O}), 1592,1505,1310,1243,1192,1144$, 1016, 866, 770. - ${ }^{1} \mathrm{H}-\mathrm{NMR}\left(250 \mathrm{MHz}, \mathrm{CDCl}_{3}\right): \delta=1.02\left(\mathrm{t},{ }^{3} J=7.5 \mathrm{~Hz}, 3 \mathrm{H}, \mathrm{CH}_{3}\right), 1.03\left(\mathrm{t},{ }^{3} J\right.$ $\left.=7.5 \mathrm{~Hz}, 3 \mathrm{H}, \mathrm{CH}_{3}\right), 1.691 .02\left(\mathrm{t},{ }^{3} \mathrm{~J}=3.0 \mathrm{~Hz}, 1 \mathrm{H}, 6-\mathrm{H}\right), 1.72-1.86(\mathrm{~m}, 6 \mathrm{H}$, $\left.2 \times \mathrm{OCH}_{2} \mathrm{CH}_{2} \mathrm{CH}_{3}, 1-\mathrm{H}, 5-\mathrm{H}\right), 2.16(\mathrm{~m}, 2 \mathrm{H}, 2-\mathrm{H}, 4-\mathrm{H}), 2.22-2.49$ (m, $\left.2 \mathrm{H}, 2-\mathrm{H}, 4-\mathrm{H}\right), 2.62-$ 2.78 (quin, $\left.{ }^{3} \mathrm{~J}=6.5 \mathrm{~Hz}, 1 \mathrm{H}, 3-\mathrm{H}\right), 3.82-3.93\left(\mathrm{~m}, 4 \mathrm{H}, 2 \times \mathrm{OCH}_{2} \mathrm{CH}_{2} \mathrm{CH}_{3}\right), 6.68-7.05(\mathrm{~m}$, $8 \mathrm{H}, \mathrm{Ar}-\mathrm{H}) . \quad-{ }^{13} \mathrm{C}-\mathrm{NMR}\left(62.9 \mathrm{MHz}, \mathrm{CDCl}_{3}, \mathrm{DEPT}\right): \delta=10.4(+) 2 \times \mathrm{CH}_{3}, 2 \times 22.1(+) \mathrm{C}-3$, $22.5(-) 2 \times \mathrm{CH}_{2}, 28.5(+) \mathrm{C}-1, \mathrm{C}-5,31.4(-) \mathrm{C}-2, \mathrm{C}-4,39.4(+) \mathrm{C}-6,69.8(-) \mathrm{OCH}_{2}, 70.2(-)$ $\mathrm{OCH}_{2}, 115.0(+) 2 \times \mathrm{Ar}-\mathrm{C}, 115.5(+) 2 \times \mathrm{Ar}-\mathrm{C}, 115.9(+) 2 \times \mathrm{Ar}-\mathrm{C}, 122.0(+) \mathrm{Ar}-\mathrm{C}, 122.2(+) \mathrm{Ar}-$ $\mathrm{C}, 143.8$ Ar- $\mathrm{C}_{\text {quart, }}, 143.9 \mathrm{Ar}-\mathrm{C}_{\text {quart }}, 149.3 \mathrm{Ar}-\mathrm{C}_{\text {quart }}, 153.2 \mathrm{Ar}-\mathrm{C}_{\text {quart }}, 156.8 \mathrm{Ar}-\mathrm{C}_{\text {quart }}, 172.3$ $\mathrm{C}=\mathrm{O}, 173.6 \mathrm{C}=\mathrm{O} .-\mathrm{C}_{30} \mathrm{H}_{38} \mathrm{O}_{4}$ (438.53): ber. C 71.21, H 6.90\%; gem. C 71.07, H 6.89\%.

Ethyldiazoacetat (37): In einem 2-1 Dreihalskolben mit KPG-Rührer, Tropftrichter und Innenthermometer wurden zu einer Lösung von $140 \mathrm{~g}$ $(1.00 \mathrm{Mol})$ Glycinethylester Hydrochlorid in $250 \mathrm{ml}$ Wasser und $600 \mathrm{ml}$ $\mathrm{CH}_{2} \mathrm{Cl}_{2}$ unter Stickstoff bei $-5^{\circ} \mathrm{C}$ Innentemperatur $83.0 \mathrm{~g}$ (1.20 Mol, $\left.1.2 \mathrm{äq}\right) \mathrm{NaNO}_{2}$ in

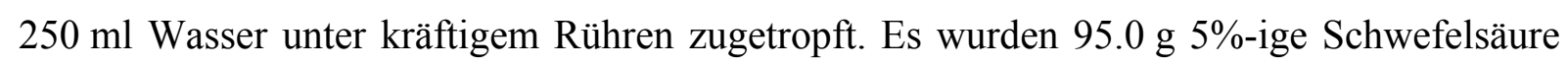
während 3 min zugetropft. Nach beendeter Gasentwicklung wurde die Reaktionsmischung in einen eiskalten 2-1 Scheidetrichter überführt, die organische Phase auf 11 eiskalte 5\%-ige

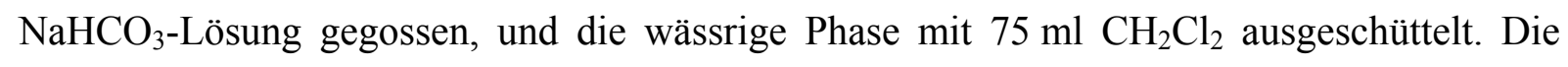
wässrige Phase wurde mit Indikatorpapier auf Säurefreiheit geprüft. Wenn noch Säure vorhanden war, musste die Lösung so lange mit $\mathrm{NaHCO}_{3}$-Lösung geschüttelt werden, bis alle Säurespuren entfernt waren. Die vereinigten organischen Phasen wurden mit $\mathrm{NaSO}_{4}$ getrocknet und das Lösungsmittel unter vermindertem Druck entfernt. Es wurden $95.0 \mathrm{~g}$ (833 mmol, $83 \%$ ) der Zielverbindung isoliert, die für weitere Synthesen nicht destilliert werden musste. ${ }^{1} \mathrm{H}-\mathrm{NMR}\left(250 \mathrm{MHz}, \mathrm{CDCl}_{3}\right): \delta=1.30\left(\mathrm{t},{ }^{3} J=8.0 \mathrm{~Hz}, 3 \mathrm{H}, \mathrm{CH}_{3}\right), 4.20\left(\mathrm{q},,{ }^{3} J=\right.$ $\left.8.0 \mathrm{~Hz}, 2 \mathrm{H}, \mathrm{CH}_{2}\right), 4.75\left(\mathrm{~s}, 1 \mathrm{H}, \mathrm{HC}=\mathrm{N}_{2}\right)$. Die physikalischen Eigenschaften stimmen mit den Literaturangaben überein. 
Cinnamylacetoacetat (52): Es wurde eine Lösung von 13.87 g (104 mmol) Zimtalkohol und Tributylphosphin (10 mol\%, $2.09 \mathrm{~g}, 10.4 \mathrm{mmol})$ in $150 \mathrm{ml}$ trockenem $\mathrm{CH}_{2} \mathrm{Cl}_{2}$ in einem $500 \mathrm{ml}$ Dreihalskolben vorgelegt, und 17.4 g (207 mmol, 1 äq) frisch destilliertes Diketen so schnell zugegeben, dass die Lösung nicht zu stark siedete. Nach beendeter Zugabe wurde die

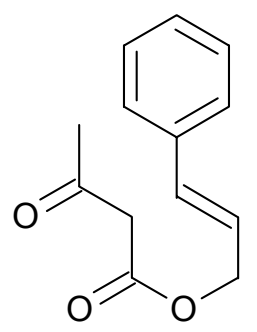
Reaktionsmischung $3 \mathrm{~h}$ unter Rückfluss erwärmt. Nach Abkühlen wurde überschüssiges Diketen mit $50 \mathrm{ml}$ Wasser gequencht, $15 \mathrm{~min}$ kräftig gerührt, die Phasen getrennt, und das Tributylphospin durch Ausschütteln mit $2 \times 200 \mathrm{ml} 1 \mathrm{~N} \mathrm{H}_{2} \mathrm{SO}_{4}$ in die wässrige Phase überführt. Saure Nebenprodukte wurden durch Extraktion mit $100 \mathrm{ml}$ 5\%-iger $\mathrm{NaOH}-L o ̈ s u n g$ entfernt. Die wässrigen Phasen wurden mit $100 \mathrm{ml}$ Hexan extrahiert. Die vereinigten organischen Phasen wurden mit $\mathrm{MgSO}_{4}$ getrocknet, das Lösungsmittel unter vermindertem Druck entfernt, und das Rohprodukt durch Säulenchromatographie (240 g Kieselgel, Säule $25 \times 5 \mathrm{~cm}$, Laufmittel Ethylacetat:Hexan 8.5:1.5) gereinigt. Es wurden 20.0 g (88\%) der Zielverbindung erhalten. . - ${ }^{1} \mathrm{H}-\mathrm{NMR}\left(250 \mathrm{MHz}, \mathrm{CDCl}_{3}\right): \delta=2.29(\mathrm{~s}, 3 \mathrm{H}$, $\left.\mathrm{CH}_{3}\right), 3.50\left(\mathrm{~s}, 2 \mathrm{H}, \mathrm{CH}_{2}\right), 4.82\left(\mathrm{~d},{ }^{3} \mathrm{~J}=1.5 \mathrm{~Hz}, 2 \mathrm{H}, \mathrm{CH}_{2}\right), 6.25\left(\mathrm{dt},{ }^{3} J=1.5 \mathrm{~Hz}, 16 \mathrm{~Hz}, 1 \mathrm{H}\right.$, $\mathrm{CH}), 6.68\left(\mathrm{~d},{ }^{3} J=16 \mathrm{~Hz} 1 \mathrm{H}, \mathrm{CH}\right), 7.32(\mathrm{~m}, 5 \mathrm{H}, \mathrm{Ar}-\mathrm{H})-{ }^{13} \mathrm{C}-\mathrm{NMR}\left(62.9 \mathrm{MHz}, \mathrm{CDCl}_{3}\right.$, DEPT): $\delta=30.2(+) \mathrm{CH}_{3}, 50.0(-) \mathrm{CH}_{2}, 65.9(+), 122.3(+) \mathrm{CH}, 126.6(+) \mathrm{Ar}-\mathrm{C}, 128.2(+)$ Ar-C, $128.6(+)$ Ar-C, $134.9(+) \mathrm{CH}, 136.0(+)$ Ar-C, $166.9(\mathrm{C}=\mathrm{O}), 200.7(\mathrm{C}=\mathrm{O})$.

Cinnamyldiazoacetat (53): $\quad$ Es $\quad$ wurden $\quad 10.9 \mathrm{~g} \quad(50.0 \mathrm{mmol})$ Cinnamylacetoacetat 52 und $9.85 \mathrm{~g}$ (50.0 mmol, 1.0 eq) Tosylazid und $386 \mathrm{mg}$ Tetrabutyl-ammoniumbromid in $200 \mathrm{ml} \mathrm{CH}_{2} \mathrm{Cl}_{2}$ gelöst und $30 \mathrm{~min}$ gerührt. Dann wurden $10 \mathrm{ml} 10 \mathrm{~N}$ NaOH-Lösung zugegeben und die Reaktionsmischung $12 \mathrm{~h}$ bei Raumtemperatur gerührt. Die Lösung wurde

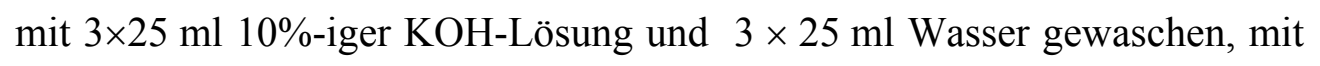<smiles>N#CC(=O)OC/C=C/c1ccccc1</smiles>
$\mathrm{NaSO}_{4}$ getrocknet und das Lösungsmittel unter vermindertem Druck entfernt. Nach Filtration über Kieselgel (Säule $5 \times 3 \mathrm{~cm}$, Laufmittel Hexan: $\mathrm{CH}_{2} \mathrm{Cl}_{2}$ 1:1) wurden $5.00 \mathrm{~g}$ (24.7 mmol, $49 \%)$ der Zielverbindung isoliert. - IR (KBr): $v\left[\mathrm{~cm}^{-1}\right]=3115,3082,2111\left(\mathrm{CN}_{2}\right), 1695$ $(\mathrm{C}=\mathrm{O}), 1391,1353,1239,1177,967,739 .-{ }^{1} \mathrm{H}-\mathrm{NMR}\left(250 \mathrm{MHz}, \mathrm{CDCl}_{3}\right): \delta=4.79(\mathrm{bs}, 1 \mathrm{H}$, $\left.\mathrm{CH}=\mathrm{N}_{2}\right), 4.82\left(\mathrm{dd},{ }^{3} J=7.0 \mathrm{~Hz},{ }^{4} J=1.5 \mathrm{~Hz}, 2 \mathrm{H}, \mathrm{CH}_{2}\right), 6.30\left(\mathrm{dt},{ }^{3} J=7.0 \mathrm{~Hz}, 16.0 \mathrm{~Hz}, 2 \mathrm{H}\right.$, $\left.\mathrm{CH}_{2}\right), 6.65\left(\mathrm{bd},{ }^{3} \mathrm{~J}=16.0 \mathrm{~Hz}, 1 \mathrm{H}, \mathrm{CH}\right), 7.34(\mathrm{~m}, 5 \mathrm{H}, \mathrm{Ar}-\mathrm{H})-{ }^{13} \mathrm{C}-\mathrm{NMR}\left(62.9 \mathrm{MHz}, \mathrm{CDCl}_{3}\right.$, DEPT): $\delta=65.4(-) \mathrm{CH}_{2}, 123.1(+) \mathrm{CH}, 126.6(+)$ Ar-C, $128.1(+) \mathrm{Ar}-\mathrm{C}, 128.2(+) \mathrm{Ar}-\mathrm{C}$, 
$134.3(+) \mathrm{CH}, 136.1$ Ar- $\mathrm{C}_{\text {quart }}, 166.6 \mathrm{C}=\mathrm{O} . \quad-\mathrm{MS}(70 \mathrm{eV}, \mathrm{EI}), m / z(\%): 202(15)\left[\mathrm{M}^{+}\right], 173$ (10) $\left[\mathrm{M}^{+}-\mathrm{N}_{2}\right] 129(75), 117(100), 91(40)$.

2-Oxo-6-phenyl-3-oxabicyclo[3.1.0]hexan (54): Es wurden $3.70 \mathrm{~g}(18.3 \mathrm{mmol})$ Cinnamyldiazoacetat in $15 \mathrm{ml}$ Benzol während $12 \mathrm{~h} \mathrm{zu}$ einer refluxierenden Suspension von $7.08 \mathrm{~g} \mathrm{CuSO}_{4}$ und $200 \mathrm{mg} \mathrm{Cu}(\mathrm{acac})_{2}$ in $500 \mathrm{ml}$ Benzol mittels Spritzenpumpe zugetropft. Die Reaktionsmischung wurde weitere $2 \mathrm{~h}$ unter Rückfluss erwärmt und nach Abkühlen wurde der Niederschlag über Kieselgel (20 g Kieselgel, Säule $5 \times 3 \mathrm{~cm}$, Laufmittel Benzol) filtriert. Das Lösungsmittel

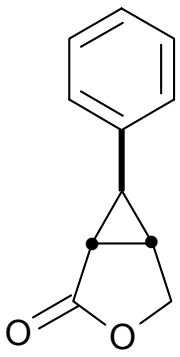
wurde unter vermindertem Druck abdestilliert, der Rückstand in $200 \mathrm{ml}$ Diethylether aufgenommen und der Niederschlag abfiltriert. Nach säulenchromatographischer Reinigung (Säule $25 \times 3 \mathrm{~cm}$, Laufmittel Petrolether : Diethylether $3: 2)$ wurden $920 \mathrm{mg}$ (5.31 mmol, $29 \%$ ) des Zielproduktes 54 isoliert. - ${ }^{1} \mathrm{H}-\mathrm{NMR}\left(250 \mathrm{MHz}, \mathrm{C}_{6} \mathrm{D}_{6}\right): \delta=1.40$ (tdd, ${ }^{3} J=3.0 \mathrm{~Hz}$, $3.5 \mathrm{~Hz}, 6.0 \mathrm{~Hz}, 1 \mathrm{H}$ ), $1.71\left(\mathrm{t},{ }^{3} \mathrm{~J}=3.5 \mathrm{~Hz}, 1 \mathrm{H}, \mathrm{CH}\right), 1.85\left(\mathrm{dd},{ }^{3} \mathrm{~J}=3.0 \mathrm{~Hz} 6.0 \mathrm{~Hz}, 1 \mathrm{H}, \mathrm{CH}\right)$, $3.52\left(\mathrm{bd},{ }^{3} \mathrm{~J}=3.5 \mathrm{~Hz}, 2 \mathrm{H}, \mathrm{CH}_{2}\right), 6.57$ (m, $\left.2 \mathrm{H}, \mathrm{Ar}-\mathrm{H}\right), 7.06(\mathrm{~m}, 3 \mathrm{H}, \mathrm{Ar}-\mathrm{H})-{ }^{13} \mathrm{C}-\mathrm{NMR}$ $\left(62.9 \mathrm{MHz}, \mathrm{CDCl}_{3}, \mathrm{DEPT}\right): \delta=26.2(+) \mathrm{CH}, 27.4(+) \mathrm{CH}, 29.4(+) \mathrm{CH}, 69.7(-) \mathrm{CH}_{2}, 125.9$ $(+) \quad A r-C, \quad 127.2 \quad(+) \quad A r-C, 128.7 \quad(+) \quad A r-C, \quad 137.2 \quad A r-C_{\text {quart }}, \quad 175.0 \quad \mathrm{C}=\mathrm{O}$. - MS (70 eV, EI), m/z (\%): 174 (52) [M+], 129 (100), 115 (70).

1-[2,3-Di(bromomethyl)cyclopropyl]benzol (56): Zu einer im Aceton/Trockeneis-Bad auf $-25^{\circ} \mathrm{C}$ gekühlten Lösung von $2.94 \mathrm{~g}$ Triphenylphosphin (11.2 mmol, 2.0 äq) wurden während $30 \mathrm{~min} 0.58 \mathrm{ml}$ Brom in $1.12 \mathrm{ml} \mathrm{CH}_{2} \mathrm{Cl}_{2}$ zugetropft und weitere $30 \mathrm{~min}$ bei $-25^{\circ} \mathrm{C}$ gerührt. Es wurden dann zu dem Komplex 1.00 g (5.61 mmol) 2-Hydroxymethyl-3-phenylcyclopropylmethanol 60 und $0.90 \mathrm{ml}$ Pyridin in $2.25 \mathrm{ml} \mathrm{CH}_{2} \mathrm{Cl}_{2}$ zugegeben, eine weitere Stunde bei

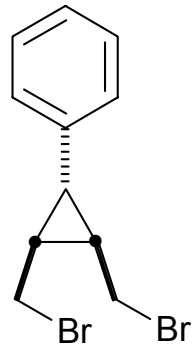
$-25^{\circ} \mathrm{C}$ und $12 \mathrm{~h}$ bei $20^{\circ} \mathrm{C}$ gerührt. Die Suspension wurde mit $20 \mathrm{ml}$ Pentan versetzt, der Niederschlag abfiltriert und mit $\mathrm{CH} 2 \mathrm{Cl} 2$ gewaschen. Die organische Phase wurde mit $\mathrm{MgSO}_{4}$ getrocknet und das Lösungsmittel unter vermindertem Druck entfernt. Es wurden $2.91 \mathrm{~g}$ Rohprodukt säulenchromatographisch (100 g Kieselgel, Säule $10 \times 2.5 \mathrm{~cm}$, Laufmittel $\mathrm{CH}_{2} \mathrm{Cl}_{2}$ ) gereinigt. Es konnten $1.25 \mathrm{~g}(74 \%)$ Zielprodukt isoliert werden, die ohne weitere Charakterisierung verwendet wurden. ${ }^{1} \mathrm{H}-\mathrm{NMR}\left(250 \mathrm{MHz}, \mathrm{CHCl}_{3}\right): \delta=2.00(\mathrm{~m}, 3 \mathrm{H}, \mathrm{CH})$, 3.60 (m, 4 H, $\left.\mathrm{CH}_{2}\right), 7.18$ (m, 2 H, Ar-H), 7.30 (m, 3 H, Ar-H). 
2-Hydroxymethyl-3-phenylcyclopropylmethanol (60): 1.02 g (5.74 mmol) 2Oxo-6-phenyl-3-oxabicyclo[3.1.0]hexan 54 wurden in $50 \mathrm{ml}$ THF gelöst und bei $20^{\circ} \mathrm{C}$ unter Rühren während $10 \mathrm{~min}$ zu einer Suspension von $163 \mathrm{mg}$ (4.29 mmol, $\quad 0.75$ äq) $\mathrm{LiAlH}_{4}$ in $30 \mathrm{ml}$ THF zugetropft. Die Reaktionsmischung wurde $3 \mathrm{~h}$ unter Rückfluss erwärmt. Nach dem Abkühlen wurden $0.2 \mathrm{ml}$ Wasser, $0.2 \mathrm{ml}$ 15\%-ige NaOH-Lösung, und dann

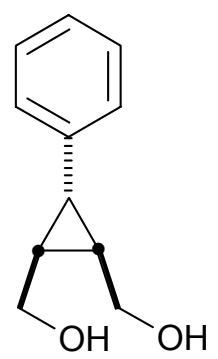
$0.6 \mathrm{ml}$ Wasser zugegeben. Der Niederschlag wurde abfiltriert und $12 \mathrm{~h}$ unter Rückfluß mit THF extrahiert. Die vereinigten organischen Phasen wurden mit $\mathrm{MgSO}_{4}$ getrocknet und das Lösungsmittel unter vermindertem Druck entfernt. Es konnten 1.02 g (99.8\%) Zielprodukt isoliert werden. ${ }^{1} \mathrm{H}-\mathrm{NMR}\left(250 \mathrm{MHz}, \mathrm{C}_{6} \mathrm{D}_{6}\right): \delta=1.52\left(\mathrm{t},{ }^{3} \mathrm{~J}=5.0 \mathrm{~Hz}, 1 \mathrm{H}, \mathrm{CH}\right), 1.66(\mathrm{~m}, 2 \mathrm{H}$, $\mathrm{CH}), 3.30\left(\mathrm{~m}, 2 \mathrm{H}, \mathrm{CH}_{2}\right), 4.05\left(\mathrm{~m}, 2 \mathrm{H}, \mathrm{CH}_{2}\right), 4.31$ (bs, $\left.2 \mathrm{H}, \mathrm{OH}\right), 6.95$ (m, 2H, Ar-H), 7.13 $(\mathrm{m}, 3 \mathrm{H}, \mathrm{Ar}-\mathrm{H})-{ }^{13} \mathrm{C}-\mathrm{NMR}\left(62.9 \mathrm{MHz}, \mathrm{CDCl}_{3}, \mathrm{DEPT}\right): \delta=27.1(+) \mathrm{CH}, 28.9(+) \mathrm{CH}, 61.7$ (-) $\quad \mathrm{OCH}_{2}, \quad 125.8(+) \mathrm{Ar}-\mathrm{C}, 126.0(+) \mathrm{Ar}-\mathrm{C}, 128.4(+) \mathrm{Ar}-\mathrm{C}, 141.5 \mathrm{Ar}-\mathrm{C}_{\text {quart }}$. - MS (70 eV, EI), m/z (\%): 178 (20) $[\mathrm{M}+], 147 \quad(22) \quad\left[\mathrm{M}^{+}-\mathrm{CH}_{2} \mathrm{OH}\right], 130$ (100) $\left[\mathrm{M}^{+}-\right.$ $\left.\mathrm{CH}_{2}(\mathrm{OH})_{2}\right], 115$ (40), 105 (20), 91 (90), 77 (20).

2-Methylsulfonyloxymethyl-3-phenylcyclopropylmethylmethanesulfonat (61): $\mathrm{Zu}$ einer Lösung von $1.00 \mathrm{~g} \quad$ (5.61 mmol) 2-Hydroxymethyl-3phenylcyclopropylmethanol 60 und $1.70 \mathrm{~g}$ (16.8 mmol, 3 äq) Triethylamin in $30 \mathrm{ml} \mathrm{CH}_{2} \mathrm{Cl}_{2}$ wurden unter Eiskühlung $1.04 \mathrm{~g}$ (12.3 mmol, 2.2äq) Mesylchlorid zugetropft und $15 \mathrm{~min}$ bei $-10{ }^{\circ} \mathrm{C}$ gerührt. Die Reaktionsmischung wurde mit $10 \mathrm{ml}$ Eiswasser, mit $10 \mathrm{ml} 10 \%$-iger

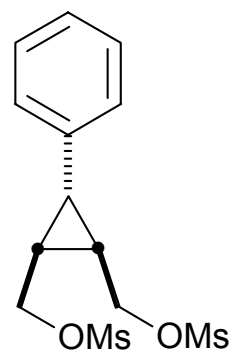
Salzsäure, mit $10 \mathrm{ml}$ gesättiger $\mathrm{NaHCO}_{3}$-Lösung und mit $10 \mathrm{ml}$ gesättigter wässriger Kochsalzlösung gewaschen, mit $30 \mathrm{ml} \mathrm{CH}_{2} \mathrm{Cl}_{2}$ verdünnt, mit $\mathrm{MgSO}_{4}$ getrocknet und das Lösungsmittel unter vermindertem Druck entfernt. Es wurden $1.87 \mathrm{~g}$ (5.61 mmol, 100\%) des Zielproduktes isoliert, die ohne weitere Charakterisierung in der Folgereaktion eingesetzt wurden.

3-nPropyl-1,5-dibrompentan (70a): Zu $43.1 \mathrm{~g}$ (164 mmol, 2.0 äq) Triphenylphosphin in $800 \mathrm{ml}$ trockenem $\mathrm{CH}_{2} \mathrm{Cl}_{2}$ wurden bei $-25{ }^{\circ} \mathrm{C}$ unter Rühren während 30 min $26.2 \mathrm{~g}$ (164 mmol, 2.0 äq) Brom zugetropft. Nach weiteren 30 min Rühren wurde $12.0 \mathrm{~g}(82.1 \mathrm{mmol}$,

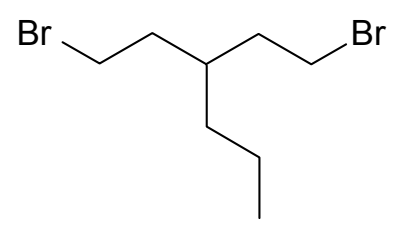
1.0 äq) 3-n-Propyl-1,5-pentadiol in einer Lösung von $6.39 \mathrm{~g}$ (82.1 mmol, 1.0 äq) Pyridin in 
$17.0 \mathrm{ml} \mathrm{CH}_{2} \mathrm{Cl}_{2}$ bei $-30{ }^{\circ} \mathrm{C}$ zugetropft. Es wurde $24 \mathrm{~h}$ bei Raumtemperatur gerührt, das Lösungsmittel unter vermindertem Druck entfernt, der Rückstand zweimal $2 \mathrm{~h}$ mit $250 \mathrm{ml}$ Hexan gerührt, die organische Phase nach Filtration über $\mathrm{MgSO}_{4}$ getrocknet, und das Hexan unter vermindertem Druck entfernt. Man erhielt $17.2 \mathrm{~g}$ Rohprodukt, das nach Kugelrohrdestillation $\left(135^{\circ} \mathrm{C}, 10^{-2}\right.$ Torr) 13.4 (49.3 mmol, $60 \%$ ) des Zielproduktes ergab. IR (KBr): $v\left[\mathrm{~cm}^{-1}\right]=3013,2961,2929,2869,1732,1453,655(\mathrm{C}-\mathrm{Br}), 567(\mathrm{C}-\mathrm{Br})-{ }^{1} \mathrm{H}-\mathrm{NMR}$ $\left(250 \mathrm{MHz}, \mathrm{CDCl}_{3}\right): \delta=0.91\left(\mathrm{t}, 3 \mathrm{~J}=6.5 \mathrm{~Hz}, 3 \mathrm{H}, \mathrm{CH}_{3}\right), 1.29\left(\mathrm{~m}, 4 \mathrm{H}, \mathrm{CH}_{2}\right), 1.77(\mathrm{~m}, 1 \mathrm{H}$, $\mathrm{CH}), 1.85\left(\mathrm{~m}, 4 \mathrm{H}, \mathrm{CH}_{2}\right), 3.42\left(\mathrm{t},{ }^{3} \mathrm{~J}=7.0 \mathrm{~Hz}, 4 \mathrm{H}, \mathrm{CH}_{2}\right) .-{ }^{13} \mathrm{C}-\mathrm{NMR}\left(62.9 \mathrm{MHz}, \mathrm{CDCl}_{3}\right.$, DEPT): $\delta=14.3(+) \mathrm{CH}_{3}, 19.3(-) \mathrm{CH}_{2}, 31.3(-) \mathrm{CH}_{2}, 34.7$ (+) $\mathrm{CH}, 35.3(-) \mathrm{CH}_{2}, 36.6(-)$ $\mathrm{CH}_{2 .}$ - MS (70 eV, EI), m/z (\%): 272 (2) $\left[\mathrm{M}^{+}\right], 193$ (5) $\left[\mathrm{M}^{+}-\mathrm{HBr}\right], 150$ (15) $\left[\mathrm{M}^{+}-\mathrm{HBr}-\right.$ $\left.\mathrm{C}_{2} \mathrm{H}_{5}\right], 111(30)\left[\mathrm{M}^{+}-\mathrm{HBr}-\mathrm{Br}\right], 83(60)\left[\mathrm{C}_{6} \mathrm{H}_{11}{ }^{+}\right], 69(55)\left[\mathrm{C}_{5} \mathrm{H}_{9}{ }^{+}\right], 55(99)\left[\mathrm{C}_{4} \mathrm{H}_{7}^{+}\right], 41$ (100) $\left[\mathrm{C}_{3} \mathrm{H}_{5}^{+}\right]$.

2-(2-Cyanomethyl-3-phenylcyclopropyl)acetonitril (71): Es wurden $1.06 \mathrm{~g}$ (3.49 mmol) 1-[2,3-Di(bromomethyl)cyclopropyl]benzol $56 \quad 920 \mathrm{mg}$ (13.9 mmol, 4 äq) KCN und $46.0 \mathrm{mg}$ ( $0.17 \mathrm{mmol}, 5 \mathrm{Mol} \%$ ) 18-Krone-6 in $10 \mathrm{ml}$ THF bei $20^{\circ} \mathrm{C}$ unter DC-Kontrolle $7 \mathrm{~d}$ gerührt und $2 \mathrm{~d}$ unter Rückfluss erwärmt. Nach Abkühlen wurde die Reaktionsmischung mit $50 \mathrm{ml}$ Diethylether verdünnt, mit $2 \times 50 \mathrm{ml}$ Wasser ausgeschüttelt, die wässrige Phase mit $50 \mathrm{ml}$

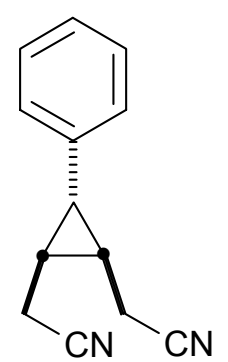
Diethylether zurückextrahiert, und die vereinigten organischen Phasen mit $\mathrm{MgSO}_{4}$ getrocknet. Nach säulenchromatographischer Reinigung an Kieselgel (60 g Kieselgel, Säule $10 \times 2 \mathrm{~cm}$, Laufmittel Petrolether: $\mathrm{CH}_{2} \mathrm{Cl}_{2}$ 1:1) konnten $250 \mathrm{mg}$ (36\%) des Zielproduktes isoliert werden. ${ }^{1} \mathrm{H}-\mathrm{NMR}\left(250 \mathrm{MHz}, \mathrm{C}_{6} \mathrm{D}_{6}\right): \delta=1.40(\mathrm{~s}, 1 \mathrm{H}, \mathrm{CH}), 2.00\left(\mathrm{dd}^{3} J=7.0 \mathrm{~Hz}, 7.0\right.$ $\left.\mathrm{Hz}, 2 \mathrm{H}, \mathrm{CH}_{2}\right), 2.35\left(\mathrm{dd},{ }^{3} J=7.0 \mathrm{~Hz}, 7.0 \mathrm{~Hz}, 2 \mathrm{H}, \mathrm{CH}_{2}\right), 2.80\left(\mathrm{~m}, 2 \mathrm{H}, \mathrm{CH}_{2}\right), 7.30(\mathrm{~m}, 5 \mathrm{H}$, Ar-H). 
3-nPropyl-1,5-pentadiol (71a): Es wurden $9.57 \mathrm{~g} \quad \mathrm{LiAlH}_{4}$ (252 mmol, 2 äq) in $50 \mathrm{ml}$ THF vorgelegt und auf $0{ }^{\circ} \mathrm{C}$ gekühlt. Während $2 \mathrm{~h}$ wurden $21.9 \mathrm{~g}$ (126 mmol) 3-nPropyl-1,5pentandisäure in $50 \mathrm{ml}$ THF zugetropft. Nach beendeter Zugabe<smiles>CCCC(CCO)CCO</smiles>
wurde die Reaktionsmischung für $20 \mathrm{~h}$ unter Rückfluss erwärmt. Nach Abkühlen wurden langsam $10 \mathrm{ml}$ Wasser, $15 \mathrm{ml}$ 15\%-ige $\mathrm{NaOH}$-Lösung und nachfolgend $10 \mathrm{ml}$ Wasser zugegeben und 30 min gerührt. Der Niederschlag wurde abfiltriert, $1 \mathrm{~h}$ mit $200 \mathrm{ml}$ siedendem THF extrahiert. Die vereinigten organischen Phasen wurden mit $\mathrm{MgSO}_{4}$ getrocknet und das Lösungsmittel unter vermindertem Druck entfernt. Man erhielt $14.3 \mathrm{~g}$ (97.8 mmol, 78 \%) des Zielproduktes 71a. Das Zielprodukt wies eine für weitere Synthesen ausreichende Reinheit auf. - ${ }^{1} \mathrm{H}-\mathrm{NMR}\left(250 \mathrm{MHz}, \mathrm{CDCl}_{3}\right): \delta=0.875\left(\mathrm{t},{ }^{3} \mathrm{~J}=7.0 \mathrm{~Hz}, 3 \mathrm{H}, \mathrm{CH}_{3}\right), 1.27\left(\mathrm{~m}, 4 \mathrm{H}, \mathrm{CH}_{2}\right)$, $1.52\left(\mathrm{~m}, 4 \mathrm{H}, \mathrm{CH}_{2}\right), 1.60$ (m, $\left.1 \mathrm{H}, \mathrm{CH}\right), 3.08$ (bs, 2H, OH), $3.64\left(\mathrm{~m}, 4 \mathrm{H}, \mathrm{CH}_{2}\right) .-{ }^{13} \mathrm{C}-\mathrm{NMR}$ (62.9 MHz, $\mathrm{CDCl}_{3}$, DEPT): $\delta=14.04(+) \mathrm{CH}_{3}, 19.61$ (-) $\mathrm{CH}_{2}, 30.75$ (-) $\mathrm{CH}_{2}, 36.38$ (+) $\mathrm{CH}$, $36.80(-) \mathrm{CH}_{2}, 60.57(\mathrm{COH})$. . - MS (70 eV, DCI), $m / z(\%): 164(100)\left[\mathrm{M}+\mathrm{NH}_{4}{ }^{+}\right], 147$ (5) $\left[\mathrm{M}+\mathrm{H}^{+}\right]$

4-Bromzimtsäure (73): Es wurden $100 \mathrm{~g}$ 4-Bromzimtaldehyd (540 mmol) und $112 \mathrm{~g}$ (1.08 mol) Malonsäure bei $50{ }^{\circ} \mathrm{C}$ in $216 \mathrm{ml}$ Pyridin gelöst und $8.1 \mathrm{ml}$ Piperidin zugegeben. Die Reaktionsmischung wurde innerhalb von 30 min auf $80^{\circ} \mathrm{C}$ erwärmt, dann für $1 \mathrm{~h}$ auf $85^{\circ} \mathrm{C}$ gehalten und weitere $3 \mathrm{~h}$ bei einer Temperatur von $109^{\circ} \mathrm{C}-115{ }^{\circ} \mathrm{C}$ unter Rückfluss erwärmt. Nach Abkühlen wurde die Reakitonsmischung auf 2.161 kaltes Wasser gegossen und mit CO $270 \mathrm{ml} \mathrm{konz.} \mathrm{Salzsäure} \mathrm{angesäurert.} \mathrm{Die} \mathrm{ausgefallenen} \mathrm{weißen} \mathrm{Kristalle} \mathrm{wurden} \mathrm{abfiltriert,}$ mit $4 \times 80 \mathrm{ml}$ Wasser gewaschen und $3 \mathrm{~d}$ im Vakuumtrockenschrank bei $45^{\circ} \mathrm{C}$ getrocknet. Es konnten $130 \mathrm{~g}(100 \%)$ 4-Bromzimtsäure isoliert werden. . - IR $(\mathrm{KBr}): v\left[\mathrm{~cm}^{-1}\right]=2885$, 2837, 2599, $1705(\mathrm{C}=\mathrm{O}), 1627(\mathrm{C}=\mathrm{C}), 1486,1427,1334,1307,1283,1228,1069,982,817$, 708, 548, 488. - ${ }^{1} \mathrm{H}-\mathrm{NMR}(250 \mathrm{MHz}, \mathrm{DMSO}): \delta=3.50-5.00\left(\mathrm{bs}, 1 \mathrm{H}, \mathrm{CO}_{2} \mathrm{H}\right), 6.55\left(\mathrm{~d},{ }^{3} J=\right.$ $16.0 \mathrm{~Hz}, 1 \mathrm{H}, \mathrm{CH}), 7.55\left(\mathrm{~d},{ }^{3} J=16.0 \mathrm{~Hz}, 1 \mathrm{H}, \mathrm{CH}\right), 7.61(\mathrm{~m}, 4 \mathrm{H}, \mathrm{Ar}-\mathrm{H})-{ }^{13} \mathrm{C}-\mathrm{NMR}$ (62.9 MHz, DMSO, DEPT): $\delta=120.3(+) \mathrm{CH}, 123.7$ Ar-C $\mathrm{C}_{\text {quart }}, 130.3(+) \mathrm{Ar}-\mathrm{C}, 132.0 \mathrm{Ar}-$ $\mathrm{C}_{\text {quart }}, 133.7(+) \mathrm{Ar}-\mathrm{C}, 142.8(+) \mathrm{CH}, 167.6\left(\mathrm{CO}_{2} \mathrm{H}\right) .-\mathrm{MS}(70 \mathrm{eV}, \mathrm{EI}), m / z(\%): 226(100)$ $[\mathrm{M}+], 211(20), 183(20), 147(25), 102(85)$. 
4-Bromzimtsäuremethylester (74): $\quad 50.0 \mathrm{~g} \quad$ 4-Bromzimtsäure $\mathbf{7 3}$

(220 mmol) wurden in $468 \mathrm{ml}$ Methanol auf $0{ }^{\circ} \mathrm{C}$ gekühlt und es wurden 37.0 g (310 mmol, 1.4 eq) Thionylchlorid langsam zugetropft. Die 4Bromzimtsäure löste sich erst nach Erwärmen auf. Die Reaktionsmischung wurde 3 h unter Rückfluss erwärmt. Das Zielprodukt krisallisiert beim Abkühlen als weiße Nadeln aus. Durch Einengen der

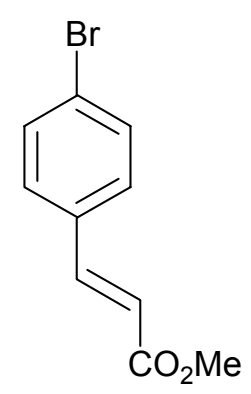
Mutterlauge kann die Ausbeute verbessert werden. Es konnten 53.1 g (219 mmol, 99.5\%) 4Bromzimtsäuremethylester isoliert werden. - IR (KBr): $v\left[\mathrm{~cm}^{-1}\right]=3061,3032,2995,1714$ $(\mathrm{C}=\mathrm{O}), 1631(\mathrm{C}=\mathrm{C}), 1489,1317,1169,1070,1006,8836,816 .-{ }^{1} \mathrm{H}-\mathrm{NMR}(250 \mathrm{MHz}$, $\left.\mathrm{CDCl}_{3}\right): \delta=3.80\left(\mathrm{~s}, 3 \mathrm{H}, \mathrm{CH}_{3}\right), 6.41\left(\mathrm{~d},{ }^{3} J=20.0 \mathrm{~Hz}, 1 \mathrm{H}, \mathrm{CH}\right), 7.37\left(\mathrm{~d},{ }^{3} J=7.5 \mathrm{~Hz}, 2 \mathrm{H}\right.$, Ar-H), $7.51\left(\mathrm{~d},{ }^{3} J=7.5 \mathrm{~Hz}, 2 \mathrm{H}, \mathrm{Ar}-\mathrm{H}\right), 7.61\left(\mathrm{~d},{ }^{3} J=20.0 \mathrm{~Hz}, 1 \mathrm{H}, \mathrm{CH}\right)-{ }^{13} \mathrm{C}-\mathrm{NMR}$ (62.9 MHz, $\left.\mathrm{CDCl}_{3}, \mathrm{DEPT}\right): \delta=51.8(+) \mathrm{OCH}_{3}, 118.4(+) \mathrm{CH}, 124.5 \mathrm{Ar}-\mathrm{C}_{\text {quart }}, 129.4(+) \mathrm{Ar}-$

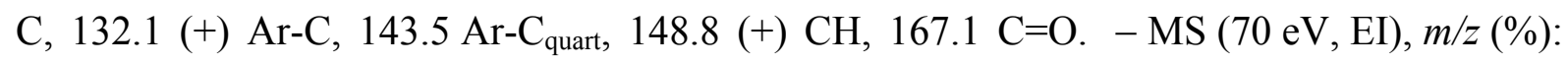
$240(90)[\mathrm{M}+], 209$ (100), $\left.\mathrm{M}^{+}-\mathrm{OMe}\right], 183(30), 102(90)$.

4-Bromzimtalkohol (75): $400 \mathrm{ml}$ (480 mmol, 3.5 eq) einer 1.2 M Lösung von DIBAH in Toluol wurden zu einer Lösung von $33.20 \mathrm{~g}$ (137 mmol) 4Bromzimtsäuremethylester in $380 \mathrm{ml}$ THF bei $-78{ }^{\circ} \mathrm{C}$ zugegeben. Nach $1 \mathrm{~h}$ Rühren bei $-78{ }^{\circ} \mathrm{C}$ wurde die Reaktionsmischung mit $100 \mathrm{ml}$ Methanol gequencht, $50 \mathrm{ml}$ Wasser zugegeben und $12 \mathrm{~h}$ stehen gelassen. Die Reaktionsmischung wurde mit $6 \mathrm{~N} \mathrm{HCl}$ auf $\mathrm{pH} 2$ gebracht um das

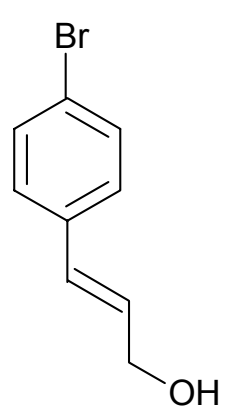
Aluminiumhydroxid in Lösung zu bringen und mit $3 \times 300 \mathrm{ml}$ Diethylether ausgeschüttelt. Die vereinigten organischen Phasen wurden mit $\mathrm{MgSO}_{4}$ getrocknet und das Lösungsmittel unter vermindertem Druck entfernt. Es wurden 25.7 g (88\%) 4-Bromzimtalkohol 75 isoliert. $-{ }^{1} \mathrm{H}-\mathrm{NMR}\left(250 \mathrm{MHz}, \mathrm{CDCl}_{3}\right): \delta=1.85(\mathrm{bs}, 1 \mathrm{H}, \mathrm{OH}), 4.30\left(\mathrm{~d},{ }^{3} \mathrm{~J}=5.0 \mathrm{~Hz}, 2 \mathrm{H}, \mathrm{CH}_{2}\right), 6.44$ $\left(\mathrm{dt},{ }^{3} J=16,5.0 \mathrm{~Hz}, 1 \mathrm{H}, \mathrm{CH}\right), 6.55\left(\mathrm{~d},{ }^{3} J=16.0 \mathrm{~Hz}, 1 \mathrm{H}, \mathrm{CH}\right), 7.22\left(\mathrm{~d},{ }^{3} J=8.5 \mathrm{~Hz}, 2 \mathrm{H}, \mathrm{Ar}-\right.$ H), $7.43\left(\mathrm{~d},{ }^{3} J=8.5 \mathrm{~Hz}, 2 \mathrm{H}, \mathrm{Ar}-\mathrm{H}\right)-{ }^{13} \mathrm{C}-\mathrm{NMR}\left(62.9 \mathrm{MHz}, \mathrm{CDCl}_{3}\right.$, DEPT): $\delta=63.5(-)$ $\mathrm{CH}_{2}, 121.4$ Ar-C $\mathrm{C}_{\text {quart }}, 128.0$ (-) Ar-C, 129.3 (-) CH, 129.7 (-)Ar-C, 131.7 (-) CH, 135.6 Ar$\mathrm{C}_{\text {quart. }}$ 
4-Bromcinnamylacetoacetat (76): Zu einer Lösung von $28.0 \mathrm{~g}$ (131 mmol) 4-Bromzimtalkohol und $200 \mathrm{mg}$ Natriummethanolat in $400 \mathrm{ml}$ Dichlormethan wurden $22.9 \mathrm{~g}$ (263 mmol, $2.0 \mathrm{eq})$ Diketen so langsam zugetropft, dass die Temperatur $30{ }^{\circ} \mathrm{C}$ nicht überstieg. Die Reaktionsmischung wurde weitere $12 \mathrm{~h}$ bei $20^{\circ} \mathrm{C}$ gerührt. Die braune Reaktionslösung wurde mit $75 \mathrm{ml} 1 \mathrm{M} \mathrm{H}_{2} \mathrm{SO}_{4}, 75 \mathrm{ml}$ gesättigter $\mathrm{NaHCO}_{3}$ -

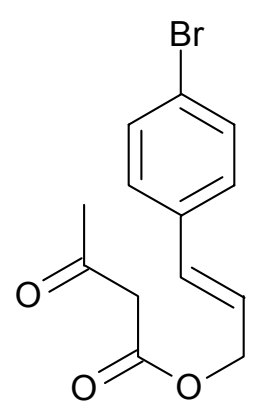
Lösung und mit 2x75 ml Wasser gewaschen. Die organische Phase wurde mit $\mathrm{MgSO}_{4}$ getrocknet, und das Lösungsmittel unter vermindertem Druck entfernt. Es konnten $34.4 \mathrm{~g}$ (88\%) Zielprodukt isoliert werden. $-{ }^{1} \mathrm{H}-\mathrm{NMR}\left(250 \mathrm{MHz}, \mathrm{CDCl}_{3}\right): \delta=2.26$ (s, $\left.3 \mathrm{H}, \mathrm{CH}_{3}\right), 3.49$ (s, $\left.2 \mathrm{H}, \mathrm{CH}_{2}\right), 4.75\left(\mathrm{~d},{ }^{3} J=6.0 \mathrm{~Hz}, 2 \mathrm{H}, \mathrm{CH}_{2}\right), 6.24\left(\mathrm{dd},{ }^{3} J=6.0,16.0 \mathrm{~Hz}\right.$, $1 \mathrm{H}, \mathrm{CH}), 6.57\left(\mathrm{~d},{ }^{3} J=16 \mathrm{~Hz}, 1 \mathrm{H}, \mathrm{CH}\right), 7.22\left(\mathrm{~d},{ }^{3} J=8.0 \mathrm{~Hz}, 2 \mathrm{H}, \mathrm{Ar}-\mathrm{H}\right), 7.41\left(\mathrm{~d},{ }^{3} J=8.0\right.$ $\mathrm{Hz}, 2 \mathrm{H}, \mathrm{Ar}-\mathrm{H})-{ }^{13} \mathrm{C}-\mathrm{NMR}\left(62.9 \mathrm{MHz}, \mathrm{CDCl}_{3}, \mathrm{DEPT}\right): \delta=30.2 \quad(+) . \mathrm{CH}_{3}, 50.0 \quad(-)$ $\mathrm{CH}_{2}, 65.6(-) \mathrm{CH}_{2}, 122.0(+)$ Ar- $\mathrm{C}_{\text {quart }}, 123.2(+) \mathrm{CH}, 128.1(+) \mathrm{Ar}-\mathrm{C}, 131.6(+) \mathrm{Ar}-\mathrm{C}, 133.3$ (+) $\mathrm{CH}, 134.9$ Ar- $\mathrm{C}_{\text {quart }}, 166.8 \mathrm{C}=\mathrm{O}, 200.5 \mathrm{C}=\mathrm{O}$.

4-Bromcinnamyldiazoacetat(77): Es wurden gemäß AAV 322.1 g (74.0 mmol) 4-Bromacetoacetat in $380 \mathrm{ml}$ Dichlormethan gelöst, $14.6 \mathrm{~g}$ (74.0 mmol, 1 eq) Tos $\mathrm{N}_{3}$ zugegeben und 30 min gerührt. Dann wurden $15 \mathrm{ml} 10 \mathrm{~N} \mathrm{NaOH}$ (150 mmol, 2.0 eq) zugegeben und $12 \mathrm{~h}$ bei RT gerührt. Es wurden $13.2 \mathrm{~g}$ (49.5 mmol, 67\%) der Titelverbindung isoliert, auf deren chromatographische Reinigung verzichtet wurde. -

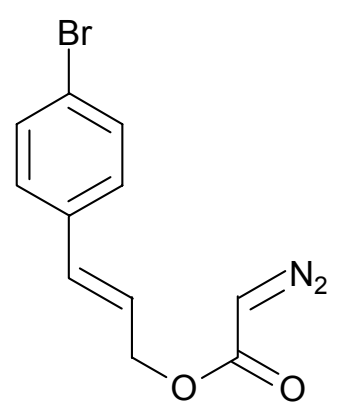
${ }^{1} \mathrm{H}-\mathrm{NMR}\left(250 \mathrm{MHz}, \mathrm{CDCl}_{3}\right): \delta=4.80\left(\mathrm{~d},{ }^{3} J=6.5 \mathrm{~Hz}, 2 \mathrm{H}, \mathrm{CH}_{2}\right), 4.85\left(\mathrm{bs}, 1 \mathrm{H}, \mathrm{HC}=\mathrm{N}_{2}\right), 6.30$ $\left(\mathrm{dt},{ }^{3} J=22.0,6.5 \mathrm{~Hz}, 1 \mathrm{H}, \mathrm{CH}\right), 6.58\left(\mathrm{~d},{ }^{3} J=22.0 \mathrm{~Hz}, 1 \mathrm{H}, \mathrm{CH}\right), 7.23\left(\mathrm{~d},{ }^{3} J=8.0 \mathrm{~Hz}, 2 \mathrm{H}\right.$, $\mathrm{Ar}-\mathrm{H}), 7.44\left(\mathrm{~d},{ }^{3} J=8.0 \mathrm{~Hz}, 2 \mathrm{H}, \mathrm{Ar}-\mathrm{H}\right)-{ }^{13} \mathrm{C}-\mathrm{NMR}\left(62.9 \mathrm{MHz}, \mathrm{CDCl}_{3}, \mathrm{DEPT}\right): \delta=46.3$ $\left(\mathrm{CN}_{2}\right), 65.1(-) \mathrm{CH}_{2}, 121.9$ Ar- $\mathrm{C}_{\text {quart }}, 124.0(+) \mathrm{CH}, 127.5(+) \mathrm{Ar}-\mathrm{C}, 131.7(+) \mathrm{Ar}-\mathrm{C}, 132.9(+)$ $\mathrm{CH}, 135.0$ Ar- $\mathrm{C}_{\text {quart }}, 168.2 \mathrm{C}=\mathrm{O}$.

Cyclopenten-4-ylbromid (81): Zu einer Lösung von (55.08 g, $210 \mathrm{mmol})$ in $250 \mathrm{ml}$ trockenem Dichlormethan wurden unter Stickstoff 33.56 g (10.82 ml, $210 \mathrm{mmol})$ Brom bei einer Temperatur von $-30--15{ }^{\circ} \mathrm{C}$ während $0.5 \mathrm{~h}$ zugetropft. Nach<smiles>BrC1CC=CC1</smiles>
weiteren 15 min Rühren wurde eine Mischung aus 93.2 g (0.95 mol) Cyclopent-3-en-1-ol 94 (16.82 g, $200 \mathrm{mmol}$ ) und $15.82 \mathrm{~g}$ trockenem Pyridin $(16.2 \mathrm{ml}, 200 \mathrm{mmol})$ bei $-15^{\circ} \mathrm{C}$ während $1 \mathrm{~h}$ zugetropft. Die Reaktionsmischung wurde weitere $12 \mathrm{~h}$ unter Schutzgas gerührt. Alle 
flüchtigen Bestandteile wurden zunächst bei 10 Torr und $30^{\circ} \mathrm{C}$ später unter 0.1 Torr bei $100^{\circ} \mathrm{C}$ in einen mit Trockeneis gekühlen Vorlagekolben umkondensiert. Die farblose umkondensierte Flüssigkeit wurde mit einer 30-cm Vigreuxkolonne feindestilliert und es konnten $26.44 \mathrm{~g}(90 \%)$ des Zielprodukts (Sdp. 61 ${ }^{\circ} \mathrm{C} @ 92$ mbar). Die ${ }^{1} \mathrm{H}$ - und ${ }^{13} \mathrm{C}-\mathrm{NMR}$ Spektren stimmen mit den Literaturangaben überein.

1-(Cyclopenten-4-yl)-4-[(trans-4-n-pentyl)cyclohexyl]benzol (89): Es wurden nach AAV $4 \quad 5.370 \mathrm{~g}$ 4-[(trans-4-n-pentyl)cyclohexyl]phenylbromid (18,53 mmol) und $6.801 \mathrm{~g}$ Cyclopenten-4-ylbromid 81 (46.32 mmol) mit $407 \mathrm{mg}$ $\mathrm{PdCl}_{2}$ (dppf) (0.56 mmol) zur Reaktion gebracht und es wurden nach säulenchromatographischer Aufarbeitung (300 g Kieselgel, Säule 40×4.5 cm, Hexan, $\left.R_{\mathrm{f}}=0.53\right) 4.10 \mathrm{~g}(75 \%)$ der Zielverbindung als farbloses Öl (Smp. $10-$ $\left.12^{\circ} \mathrm{C}\right)$ isoliert. $-{ }^{1} \mathrm{H}$ NMR: $\delta=0.98\left(\mathrm{t}, J=6.0 \mathrm{~Hz}, 3 \mathrm{H}, \mathrm{CH}_{3}\right), 1.14(\mathrm{t}, J=12.0,2$ $\mathrm{H}, \mathrm{CH}_{2}$ cyclohex.), 1.20-1.37 (m, $\left.9 \mathrm{H}\right), 1.54\left(\mathrm{td}, J=2.8,12.0,2 \mathrm{H}, \mathrm{CH}_{2}\right.$

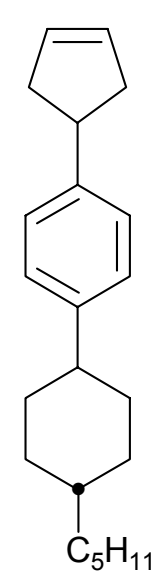
cyclohex.), 1.95 (m, 4 H, $2 \mathrm{CH}_{2}$ cyclohex.), 2.40-2.50 (m, $1 \mathrm{H}, \mathrm{CH}$ cyclohex.), 2.51 (ddd, $J=$ 2.0, 8.8, 14.5 Hz, $2 \mathrm{H}, \mathrm{CH}_{2}$ cyclopent.), 2.87 (dd, $J=8.8,14.5 \mathrm{~Hz}, 2 \mathrm{H}, \mathrm{CH}_{2}$ cyclopent.), 3.51 (p, $J=8.8 \mathrm{~Hz}, 1 \mathrm{H}, \mathrm{CH}$ cyclopent.), 5.85 (br. s, $2 \mathrm{H}, 2 \mathrm{C}=\mathrm{C}$ ), 7.15 (d, $J=8.3 \mathrm{~Hz}, 2 \mathrm{H}, \mathrm{Ar}$ ), $7.26(\mathrm{~d}, J=8.3 \mathrm{~Hz}, 2 \mathrm{H}, \mathrm{Ar}) .-{ }^{13} \mathrm{C}$ NMR: $\delta=14.1\left(\mathrm{CH}_{3}\right), 33.6,34.4,44.3\left(2 \mathrm{CH}_{2}\right), 22.7$, 26.7, 32.2, 37.4, $\left(\mathrm{CH}_{2}\right)$, 126.7, 126.8, 129.9 (2 CH), 37.3, 42.9, $44.2(\mathrm{CH}), 144.6,145.3(\mathrm{C})$. Als Nebenprodukte konnten Bis(cyclopenten-5-yl) (683 mg, $R_{\mathrm{f}}=0.58$, Öl) und 1,1'-Bis(4[(trans-4- $n$-pentyl)cyclohexyl]phenyl) $\left(101 \mathrm{mg}, R_{\mathrm{f}}=0.47\right.$, Feststoff) isoliert werden.

\section{4-[4-(3,4,5-Trifluorophenyl)phenyl]cyclopenten (90): Gemäß $\quad$ AAV 4} wurden $5.74 \mathrm{~g}$ (17.2 mmol) 1-Jod-4-(3,4,5-trifluorphenyl)benzol XX, $6.31 \mathrm{~g}$ (42.9 mmol) Cyclopenten-4-ylbromid 81 und $377 \mathrm{mg} \quad(0.52 \mathrm{mmol})$ $\mathrm{PdCl}_{2}(\mathrm{dppf})$ zur Reaktion gebracht. Es konnten nach säulenchromatographischer Aufarbeitung (250 g Kieselgel, Säule $25 \times 6 \mathrm{~cm}$, Hexan, $\left.R_{\mathrm{f}}=0.40\right) 4.17 \mathrm{~g}(98 \%)$ der Zielverbindung 90 als farbloses Öl (Smp. ca. $\left.10^{\circ} \mathrm{C}\right)$ isoliert werden. $-{ }^{1} \mathrm{H}$ NMR: $\delta=2.50(\mathrm{dd}, J=7.0,14.5 \mathrm{~Hz}, 2$ $\left.\mathrm{H}, \mathrm{CH}_{2}\right), 2.90\left(\mathrm{dd}, J=9.0,14.5 \mathrm{~Hz}, 2 \mathrm{H}, \mathrm{CH}_{2}\right), 3.54$ (tt, $J=7.0,9.0 \mathrm{~Hz}, 1 \mathrm{H}$,

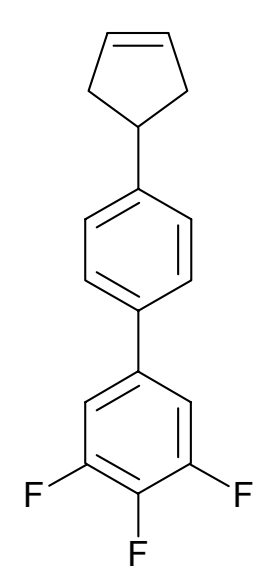
$\mathrm{CH}$ ), 5.84 (br. s, $2 \mathrm{H}, 2 \mathrm{C}=\mathrm{H}$ ), 7.19 (ddd, $J=4.5,6.5,9.0 \mathrm{~Hz}, 2 \mathrm{H}, \mathrm{Ar}$ ), 7.36 (d, $J=8.3 \mathrm{~Hz}, 2$ $\mathrm{H}, \mathrm{Ar}), 7.44(\mathrm{~d}, J=8.3 \mathrm{~Hz}, 2 \mathrm{H}, \mathrm{Ar}) .-{ }^{13} \mathrm{C} \mathrm{NMR:} \delta=41.3\left(2 \mathrm{CH}_{2}\right), 110.7$ (ddd, $J=7.1$, 14.15, 21.2 Hz), 126.7, 126.7, 129.8 (2 CH), $42.7(\mathrm{CH}), 151.5$ (ddd, $J=4.3,10.0,249.1 \mathrm{~Hz})$ 
(2 C), 135.7, $137.1(\mathrm{dt}, J=4.8,7.9 \mathrm{~Hz}) 139.0$ (dt, $J=15.5,251.2 \mathrm{~Hz}), 148.0$ (C).

\section{1-(Cyclopenten-4-yl)-4-\{trans-4-[(trans-4-n-}

propyl)cyclohexyl]cyclohexyl;benzol (91): Es wurden gemäß AAV $45.50 \mathrm{~g}$ (13.4 mmol) 4-[(trans-4-n-Pentyl)cyclohexyl]phenyljodid 5d, 4.40 g (19.9 mmol) Cyclopent-4-enylbromid 81 und $263 \mathrm{mg}$ (0.36 mmol) $\mathrm{PdCl}_{2}(\mathrm{dppf})$ zur Reaktion gebracht. Nach säulenchromatographischer Aufarbeitung (250 g Kieselgel, Säule $25 \times 6 \mathrm{~cm}$, Hexan, $\left.R_{\mathrm{f}}=0.46\right)$ konnten $4.61 \mathrm{~g}(98 \%)$ der Titelverbindung 91 als farbloser Feststoff isoliert werden (Smp. $\left.186-187^{\circ} \mathrm{C}\right) .-\mathrm{IR}(\mathrm{KBr}): \mathrm{v}\left[\mathrm{cm}^{-1}\right]=$ 2957, 2850, 1723, 1514, 1445, 1255, 1147, 978, 892, 820, 755, 697. - ${ }^{1} \mathrm{H}$ NMR: $\delta$ $=0.97\left(\mathrm{t}, J=7.0 \mathrm{~Hz}, 3 \mathrm{H}, \mathrm{CH}_{3}\right), 1.05-1.15$ (m, $2 \mathrm{H}, \mathrm{CH}_{2}$ cyclohex.), 1.20-1.30 $(\mathrm{m}, 6 \mathrm{H}), 1.33-1.54(\mathrm{~m}, 6 \mathrm{H}), 1.85-2.03(\mathrm{~m}, 9 \mathrm{H}), 2.40-2.50(\mathrm{~m}, 1 \mathrm{H}, \mathrm{CH}$ cyclohex.), 2.53 (dd, $J=7.0,14.0 \mathrm{~Hz}, 2 \mathrm{H}, \mathrm{CH}_{2}$ cyclopent.), 2.88 (dd, $J=9.0$,

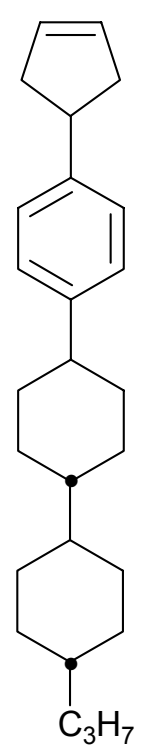
$14.0 \mathrm{~Hz}, 2 \mathrm{H}, \mathrm{CH}_{2}$ cyclopent.), 3.52 (p, J=7.9 Hz, $1 \mathrm{H}, \mathrm{CH}$ cyclopent.), 5.85 (br. s, $2 \mathrm{H}, 2$ $\mathrm{c}=\mathrm{H}), 7.21(\mathrm{~d}, J=8.3 \mathrm{~Hz}, 2 \mathrm{H}, \mathrm{Ar}), 7.26(\mathrm{~d}, J=8.3 \mathrm{~Hz}, 2 \mathrm{H}, \mathrm{Ar}) .-{ }^{13} \mathrm{C} \mathrm{NMR}: \delta=14.5\left(\mathrm{CH}_{3}\right)$, 30.1, 30.4, 33.6, 34.7, $41.3\left(2 \mathrm{CH}_{2}\right), 20.1,39.9\left(\mathrm{CH}_{2}\right), 126.8,126.9,129.9(2 \mathrm{CH}), 37.6,42.9$, 43.0, 43.4, $44.2(\mathrm{CH}), 144.7,145.4(\mathrm{C}) .-\mathrm{C}_{26} \mathrm{H}_{38}$ (350.56): calcd C 89.07, H 10.93\%; found C $89.02, \mathrm{H} 10.80 \%$.

4-(3,4,5-Trifluorophenyl)cyclopenten (92): Es wurden gemäß AAV 4 $4.22 \mathrm{~g}$ (20.0 mmol) 1- Brom-3,4,5-trifluorobenzol, $7.35 \mathrm{~g}$ (50.0 mmol) Cyclopen-4-enylbromid 81 und $439 \mathrm{mg}$ (0.6 mmol) $\mathrm{PdCl}_{2}(\mathrm{dppf})$ zur Reaktion gebracht. Es konnten nach säulenchromatographischer Aufarbeitung (300 g Kieselgel, Säule $40 \times 4.5 \mathrm{~cm}$, Hexan, $\left.R_{\mathrm{f}}=0.52\right)$ $3.27 \mathrm{~g}(82 \%)$ als farbloses Öl isoliert werden. $-{ }^{1} \mathrm{H}$ NMR: $\delta=2.37$ (ddd, $J$

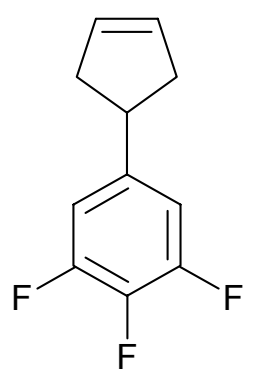
$\left.=2.0,9.0,14.5 \mathrm{~Hz}, 2 \mathrm{H}, \mathrm{CH}_{2}\right), 2.82\left(\mathrm{dd}, J=6.7,14.5 \mathrm{~Hz}, 2 \mathrm{H}, \mathrm{CH}_{2}\right), 3.39(\mathrm{tt}, J=6.3,9.0 \mathrm{~Hz}$, $1 \mathrm{H}, \mathrm{CH}), 5.76$ (br. s, $2 \mathrm{H}, 2 \mathrm{C}=\mathrm{H}), 6.84$ (ddd, $J=4.5,11.0,13.5 \mathrm{~Hz}, 2 \mathrm{H}, \mathrm{Ar}) .-{ }^{13} \mathrm{C} \mathrm{NMR}: \delta$ $=41.1\left(2 \mathrm{CH}_{2}\right), 110.6(\mathrm{ddd}, J=6.4,13.5,20.3 \mathrm{~Hz}), 129.5(2 \mathrm{CH}), 43.1(\mathrm{CH}), 151.0(\mathrm{ddd}, J=$ 4.2, 9.9, 248.6 Hz) (2 C), 137.9 (dt, $J=15.4,247.8 \mathrm{~Hz}), 143.9$ (dt, $J=4.5,9.1 \mathrm{~Hz})(\mathrm{C})$. Als Nebenprodukte konnten Bis(cyclopenten-5-yl) (145 mg, $R_{\mathrm{f}}=0.58$, Öl) und 1,1'-Bis(3,4,5trifluorphenyl) (202 mg, $R_{\mathrm{f}}=0.35$, Feststoff) isoliert werden. 
Cyclopent-3-encarbonsäure-methylester (109): $\quad 9.22 \mathrm{~g} \quad$ (80.8 mmol)

Cyclopenten-4-carbonsäure wurden mit $9.80 \mathrm{ml}$ (242 mmol, 4 äq) Methanol, $400 \mathrm{mg}$ Toluonsulfonsäure und $24.2 \mathrm{ml}$ Tetrachlorkohlenstoff $10 \mathrm{~h}$ unter Feuchtigkeitsausschluss unter Rückfluss erwärmt. Nach Abkühlen der

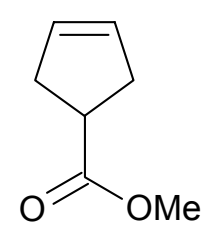
Reaktionsmischung wurde die organische Schicht mit $20 \mathrm{ml}$ Wasser, $30 \mathrm{ml}$ gesättigter $\mathrm{NaHCO}_{3}$-Lösung und gesättigter Kochsalzlösung ausgeschüttelt, mit $\mathrm{MgSO}_{4}$ getrocknet, und das Lösungsmittel im Vakuum entfernt. Es wurden $10.2 \mathrm{~g}$ (80.8 mmol, 100\%) der Zielverbindung isoliert. - IR (KBr): $v\left[\mathrm{~cm}^{-1}\right]=3059$ (s), 2957 (b), $1758(\mathrm{C}=\mathrm{O}), 1433,1366$, 1298, 1221, 1023, 798, $711-{ }^{1} \mathrm{H}-\mathrm{NMR}\left(250 \mathrm{MHz}, \mathrm{CDCl}_{3}\right): \delta=2.63\left(\mathrm{~d},{ }^{3} J=8.0 \mathrm{~Hz}, 4 \mathrm{H}\right.$, $\mathrm{CH}_{2}$ ), 3.11(quin, $\left.{ }^{3} \mathrm{~J}=8.0 \mathrm{~Hz}, 1 \mathrm{H}, \mathrm{CH}\right), 3.68\left(\mathrm{~s}, 3 \mathrm{H}, \mathrm{CH}_{3}\right), 5.65(\mathrm{~s}, 1 \mathrm{H}, \mathrm{CH}) .-{ }^{13} \mathrm{C}-\mathrm{NMR}$ (62.9 MHz, $\mathrm{CDCl}_{3}$, DEPT): $\delta=36.2(-) 2 \times \mathrm{CH}_{2}, 41.3(+) \mathrm{CH}, 51.7(+) \mathrm{OCH}_{3}, 128.8(+) \mathrm{CH}$, $176.6 \mathrm{C}_{\text {quart. }}-\mathrm{MS}(70 \mathrm{eV}, \mathrm{EI}), m / z(\%): 126(35)[\mathrm{M}+], 111(18)\left[\mathrm{M}+-\mathrm{CH}_{3}\right], 95(24)[\mathrm{M}+$ $-\mathrm{OMe}], 67$ (100) $\left[\mathrm{C}_{5} \mathrm{H}_{7}\right]$.

$\beta$-Propylglutarsäure (121): Es wurden $28.0 \mathrm{~g}$ (126 mmol) $\alpha, \alpha^{\prime}-$ Dicyano- $\beta$-propylglutardiamid in $170 \mathrm{ml} 15 \%$-iger Salzsäure aufgenommen und $5 \mathrm{~h}$ unter Rückfluß erwärmt. Nach Abkühlen<smiles>CCCC(CC(=O)O)CC(=O)O</smiles>
wurde die Disäure mit $3 \times 200 \mathrm{ml}$ Diethylether in die organische Phase überführt, mit $\mathrm{MgSO}_{4}$ getrocknet, und das Lösungsmittel unter vermindertem Druck entfernt. Das Produkt kann aus Salzsäure umkristallisiert werden, wies aber eine für die weitere Synthese ausreichende Reinheit auf. Es wurden $21.9 \mathrm{~g}$ (126 mmol, 100\%) des Zielproduktes erhalten. - ${ }^{1} \mathrm{H}-\mathrm{NMR}\left(250 \mathrm{MHz}, \mathrm{CDCl}_{3}\right): \delta=0.90\left(\mathrm{t},{ }^{3} \mathrm{~J}=8.0 \mathrm{~Hz}, 3 \mathrm{H}, \mathrm{CH}_{3}\right)$, $1.35\left(\mathrm{~m}, 4 \mathrm{H}, \mathrm{CH}_{2}\right), 2.40\left(\mathrm{~m}, 4 \mathrm{H}, \mathrm{CH}_{2}\right), 3.50$ (m, $\left.1 \mathrm{H}, \mathrm{CH}\right), 10.86$ (bs, $\left.2 \mathrm{H}, \mathrm{COOH}\right) .-{ }^{13} \mathrm{C}-$ NMR (62.9 MHz, $\left.\mathrm{CDCl}_{3}, \mathrm{DEPT}\right): \delta=13.94(+) \mathrm{CH}_{3}, 19.71(-) \mathrm{CH}_{2}, 31.57(-) \mathrm{CH}, 36.57(+)$ $\mathrm{CH}, 38.56(-) \mathrm{CH}_{2}, 179.43 \mathrm{CO}_{2} \mathrm{H}$. . - MS (70 eV, DCI), m/z (\%): $366(85)\left[2 \mathrm{M}+\mathrm{NH}_{4}^{+}\right], 209$ (15) $\left[\mathrm{M}+\mathrm{NH}_{3}+\mathrm{NH}_{4}{ }^{+}\right], 192(100)\left[\mathrm{M}+\mathrm{NH}_{4}{ }^{+}\right]$. Die physikalischen Eigenschaften stimmen mit den Literaturangaben überein. 
$\alpha, \alpha$ '-Dicyano- $\beta$-propylglutardiamid (122): 25.2 g (300 mmol, 2.00 äq) Cyanoacetamid wurden in $180 \mathrm{ml}$ Wasser aufgelöst, $10.8 \mathrm{~g}$ (150 mmol, 1.00 äq) n-Butylaldehyd zugegeben, und mit $0.3 \mathrm{ml} 3 \mathrm{~N} \mathrm{KOH}-L o ̈ s u n g$ versetzt. Nach $3 \mathrm{~h}$ hatte sich ein weißer Niederschlag gebildet. Der Niederschlag wurde<smiles>CCCC(C(N)C#N)C(C#N)C(N)=O</smiles>
abfiltriert und 3 Tage im Vakuumtrockenschrank bei $45^{\circ} \mathrm{C}$ getrocknet. Die Substanz kann aus einem Alkohol-Benzolgemisch umkristallisiert werden, war aber für weitere Synthesen ausreichend rein. Es wurden $30.0 \mathrm{~g}$ (13.5 mmol, 90\%) der Zielverbindung mit einem Schmelzpunkt von $136{ }^{\circ} \mathrm{C}$ isoliert. . - IR (KBr): $v\left[\mathrm{~cm}^{-1}\right]=3418$ (bs, $\left.\mathrm{NH}_{2}\right), 3181$ (bs, $\mathrm{NH}_{2}$ ), 2963 (s), 2247 (ss, CN), 1718 (bs), 1400 (bs), 586 (bs) - ${ }^{1} \mathrm{H}-\mathrm{NMR}$ (250 MHz, DMSO): $\delta=0.83\left(\mathrm{t},{ }^{3} J=7.5 \mathrm{~Hz}, 3 \mathrm{H}, \mathrm{CH}_{3}\right), 1.33-1.51\left(\mathrm{~m}, 4 \mathrm{H}, \mathrm{CH}_{2}\right), 2.70-2.80(\mathrm{~m}, 1 \mathrm{H}, \mathrm{CH}), 3.89$ $\left(\mathrm{d},{ }^{3} J=6.5 \mathrm{~Hz} 2 \mathrm{H}, \mathrm{CH}\right), 7.65(\mathrm{~s}, 2 \mathrm{H}, \mathrm{NH}), 7.90(\mathrm{~s}, 2 \mathrm{H}, \mathrm{NH}) .{ }^{13} \mathrm{C}-\mathrm{NMR} 14.1(+) \mathrm{CH}_{3}, 19.4$ (-) $\mathrm{CH}_{2}, 33.0(-) \mathrm{CH}_{2}, 37.4(+) \mathrm{CH}, 40.5(+) \mathrm{CH}, 117.6 \mathrm{CN}, 165.4 \mathrm{C}=\mathrm{O}$. Die physikalischen Daten stimmen mit den Literaturangaben überein.

1-(Cyclopent-3-enyl)-4-propylcyclohexanol (123): Es wurden in einem $100 \mathrm{ml}$ Dreihalskolben mit Rückflüsskühler und Septum unter Stickstoff aus $2.34 \mathrm{~g}$ (91.9 mmol, 2.05 äq) Magnesium und 12.2 g (44.8 mmol, 1.0 äq) 3-nPropyl-1,5Dibrompentan in $50 \mathrm{ml}$ THF ein Bisgrignardreagenz hergestellt. Nach $2 \mathrm{~h}$ Rühren wurden mit einer Spritzenpumpe $5.75 \mathrm{~g}$ (44.8 mmol, 1.0 äq) Cyclopen-3-

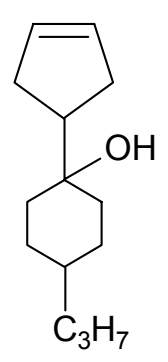
tencarbonsäuremethylester während $2 \mathrm{~h}$ zugegeben und $24 \mathrm{~h}$ gerührt. Die Reaktionsmischung wurde dann mit $\mathrm{NaHCO}_{3}$-Lösung gequencht, die wässrige von der organischen Phase getrennt, die organische Phase mit $\mathrm{MgSO}_{4}$ getrocknet, und das Lösungsmittel unter vermindertem Druck entfernt. Es konnten nach säulenchromatographischer Aufarbeitung (240 g Kieselgel, Säule $5 \times 30 \mathrm{~cm}$, Hexan:Et $2 \mathrm{O}$ 4:1, $\left.\mathrm{R}_{f}=0.3\right) \quad 5.23 \mathrm{~g}(56 \%)$ gelbes Öl isoliert werden, aus dem nach $14 \mathrm{~d}$ Stehen bei $20^{\circ} \mathrm{C}$ ein farbloser Feststoff auskristallisiert. - IR (KBr): $v\left[\mathrm{~cm}^{-1}\right]=3052,2959,1622,1461,1345$, $1293,1269,1215,1191,1163,1143,1107,1053,1016,941,915,886,848,739,702$. ${ }^{1} \mathrm{H}-\mathrm{NMR}\left(250 \mathrm{MHz}, \mathrm{CDCl}_{3}\right): \delta=0.87\left(\mathrm{t},{ }^{3} \mathrm{~J}=7.5 \mathrm{~Hz}, 3 \mathrm{H}, \mathrm{CH}_{3}\right), 1.30\left(\mathrm{~m}, 8 \mathrm{H}, \mathrm{CH}_{2}\right), 1.58$ (m, 1 H, CH), $1.60\left(\mathrm{~m}, 4 \mathrm{H}, \mathrm{CH}_{2}\right), 2.38$ (bs, $\left.4 \mathrm{H}, \mathrm{CH}_{2}\right), 2.42(\mathrm{~m}, 1 \mathrm{H}, \mathrm{CH}), 3.55$ (bs, $1 \mathrm{H}$, $\mathrm{OH}), 5.68(\mathrm{~s}, 2 \mathrm{H}, \mathrm{CH}) .-{ }^{13} \mathrm{C}-\mathrm{NMR}\left(62.9 \mathrm{MHz}, \mathrm{CDCl}_{3}, \mathrm{DEPT}\right): \delta=14.2(+) \mathrm{CH}_{3}, 20.2(-)$ $\mathrm{CH}_{2}, 28.9$ (-) $\mathrm{CH}_{2}, 32.2(-) \mathrm{CH}_{2}, 35.7$ (-) $\mathrm{CH}_{2}, 36.3(-), 37.7(+) \mathrm{CH}, 42.1(+) \mathrm{CH}$, $72.4 \mathrm{C}_{\text {quart }}$, $129.9(+) \mathrm{CH}$.

- $\mathrm{MS}(70 \mathrm{eV}, \mathrm{EI}), m / z(\%)$ : $190(25)$ 
$\left[\mathrm{M}^{+}-\mathrm{H}_{2} \mathrm{O}\right], 141(100)\left[\mathrm{M}^{+}-\mathrm{C}_{5} \mathrm{H}_{7}\right], 123(35)\left[\mathrm{M}^{+}-\mathrm{H}_{2} \mathrm{O}-\mathrm{C}_{5} \mathrm{H}_{11}\right], 67(60)\left[\mathrm{C}_{5} \mathrm{H}_{7}\right]$

4-Cyanobenzaldehydtosylhydrazon (142a): Gemäß AAV 1 wurden 12.3 g (66.1 mmol) Toluolsulfonylhydrazin in $100 \mathrm{ml}$ Methanol suspendiert und $10 \mathrm{~min}$ bei $20^{\circ} \mathrm{C}$ gerührt. Zu der Suspension wurden $10.0 \mathrm{~g}$ (76.3 mmol, 1.15 äq) 4-Cyanobenzaldehyd gegeben und weitere $10 \mathrm{~min}$ bei $20^{\circ} \mathrm{C}$ gerührt. Nach 1 min lösen sich beide Substanzen auf, und es entsteht eine gelbliche Lösung, aus der nach weiteren 2 min ein weisser Feststoff ausfällt. Es wurde 30 min<smiles></smiles>
unter Rückfluss erwärmt, das Zielprodukt aus der abgekühlten Lösung abfiltriert und das Filtrat mit $30 \mathrm{ml}$ eiskaltem Methanol gewaschen. Der Feststoff wude $1 \mathrm{~d}$ bei Raumtemperatur und $10^{-2}$ Torr von flüchtigen Substanzen befreit. Es konnten $17.7 \mathrm{~g}$ (87\%) Zielprodukt isoliert werden. - IR (KBr): $v\left[\mathrm{~cm}^{-1}\right]=3580,3430,3205,2226(\mathrm{CN}), 1595(\mathrm{~N}=\mathrm{C}), 1493,1449,1403$, 1369, 1335, 1288, 1220, 1190, 1152, 1091, 922, 846, 817, 740, 676, 539. - ${ }^{1} \mathrm{H}-\mathrm{NMR}$ (250 $\left.\mathrm{MHz}, \mathrm{C}_{6} \mathrm{D}_{6}\right): \delta=2.41\left(\mathrm{~s}, 3 \mathrm{H}, \mathrm{CH}_{3}\right), 7.33\left(\mathrm{~d},{ }^{3} J=8.5 \mathrm{~Hz}, 2 \mathrm{H}, \mathrm{Ar}-\mathrm{H}\right), 7.64(\mathrm{~m}, 4 \mathrm{H}, \mathrm{Ar}-\mathrm{H})$, $7.78(\mathrm{~s}, 1 \mathrm{H}, \mathrm{CH}), 7.87(\mathrm{~d}, 3 \mathrm{~J}=8.0 \mathrm{~Hz}, 2 \mathrm{H}, \mathrm{Ar}-\mathrm{H}), 8.63(\mathrm{~s}, 1 \mathrm{H}, \mathrm{NH}).) \quad-{ }^{13} \mathrm{C}-\mathrm{NMR}$ $\left(62.9 \mathrm{MHz}, \mathrm{CDCl}_{3}, \mathrm{DEPT}\right): \delta=21.62(+) \mathrm{CH}_{3}, 113.86 \mathrm{Ar}-\mathrm{C}_{\text {quart }}, 118.37(\mathrm{CN}), 127.4(+) \mathrm{Ar}-\mathrm{C}$, 127.8 (+) Ar-C, 129.8 (+) Ar-C, 132.3 Ar- $\mathrm{C}_{\text {quart, }}$ 134.9 (+) Ar- $\mathrm{C}_{\text {quart }}, 137.4$ Ar- $\mathrm{C}_{\text {quart }}, 144.6(+$, $\mathrm{C}=\mathrm{N})$. Die physikalischen Daten stimmen mit den Literaturangaben überein.

4-Diazomethylbenzonitril (143a): Gemäss AAV 2 wurden $860 \mathrm{mg}$ (37.4 mmol, 2.0 eq) Natrium in $15 \mathrm{ml}$ Ethylenglycol in einer $100 \mathrm{ml}$ Pyrexflasche aufgelöst und $5.00 \mathrm{~g}$ (18.7 mmol) 4-Cyanobenzaldehydtosylhydrazon eingerührt. Der zähe Brei wurde mit $50 \mathrm{ml}$ Hexan überschichtet, die Flasche gut verschlossen, und die Reaktionsmischung unter Rühren für $5 \mathrm{~min}$ auf $90^{\circ} \mathrm{C}$ erwärmt. Das<smiles>N#Cc1ccc(C=N)cc1</smiles>
Reaktionsgefäss wurde in einem Eisbad schnell abgekühlt, die rote Hexanlösung abpipettiert und in einen mit Aluminiumfolie umwickelten eisgekühlten Kolben überführt. Dieser Vorgang wurde so oft wiederholt, bis sich keine Diazokomponente mehr in der Hexanphase löste (5 x, Rotfärbung). Das Hexan wurde unter vermindertem Druck bei $20{ }^{\circ} \mathrm{C}$ entfernt und es konnten $1.79 \mathrm{~g}(67 \%)$ der Zielverbindung isoliert werden. ${ }^{1} \mathrm{H}-\mathrm{NMR}\left(250 \mathrm{MHz}, \mathrm{C}_{6} \mathrm{D}_{6}\right)$ : $\delta=5.06\left(\mathrm{~s}, 1 \mathrm{H}, \mathrm{CHN}_{2}\right), 6.95\left(\mathrm{~d},{ }^{3} J=7.5 \mathrm{~Hz}, 2 \mathrm{H}, \mathrm{Ar}-\mathrm{H}\right), 7.52\left(\mathrm{~d},{ }^{3} J=7.5 \mathrm{~Hz}, 2 \mathrm{H}, \mathrm{Ar}-\mathrm{H}\right)$. ${ }^{13} \mathrm{C}-\mathrm{NMR}\left(62.9 \mathrm{MHz}, \mathrm{CDCl}_{3}, \mathrm{DEPT}\right): \delta=63.65(+) \mathrm{CHN}_{2}, 118.5 \mathrm{CN}, 119.3 \mathrm{Ar}-\mathrm{C}_{\text {quart }}, 121.2$ (+) Ar-C, $132.7(+)$ Ar-C, 136.4 Ar-C quart. 
4-Ethoxy-2,3-difluorphenyldiazomethan (143b): Nach AAV 2 wurden $1.83 \mathrm{~g}$ (5.17 mmol) 4-Ethoxy-2,3-difluorbenzyltosylhydrazon bei $90{ }^{\circ} \mathrm{C}$ in einer Lösung von Natriumglycolat in Glycol zur Reaktion gebracht und das Reaktionsprodukt mit Hexan extrahiert. Es wurden 830 mg (5.09 mmol, 98\%) des Zielprodukts isoliert.

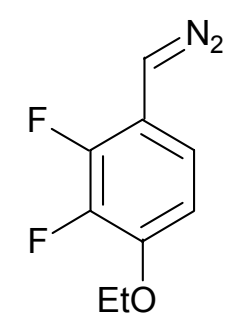
- IR (KBr): v [ cm $\left.{ }^{-1}\right]=2988$ (s), 2066 (s), 1617, 1511, 1304, 1082 (s). - ${ }^{1} \mathrm{H}-\mathrm{NMR}(250 \mathrm{MHz}$, $\left.\mathrm{CDCl}_{3}\right): \delta=1.40\left(\mathrm{t},{ }^{3} J=6.0 \mathrm{~Hz}, 3 \mathrm{H}, \mathrm{CH}_{3}\right), 4.13\left(\mathrm{q},{ }^{3} J=6.0 \mathrm{~Hz}, 2 \mathrm{H}, \mathrm{CH}_{2}\right), 5.00(\mathrm{~s}, 1 \mathrm{H}$, $\mathrm{HC}=\mathrm{N}) 6.55\left(\mathrm{t},{ }^{3} J=8.5 \mathrm{~Hz}, 1 \mathrm{H}, \mathrm{Ar}-\mathrm{H}\right), 7.70\left(\mathrm{t},{ }^{3} J=8.5 \mathrm{~Hz}, 1 \mathrm{H}, \mathrm{Ar}-\mathrm{H}\right) .-{ }^{13} \mathrm{C}-\mathrm{NMR}$ (62.9 MHz, $\mathrm{CDCl}_{3}$, DEPT): $\delta=14.4(+) \mathrm{CH}_{3}, 65.7$ (-) $\mathrm{CH}_{2}, 110.8$ (-) Ar-C, 115.5 (-) Ar-C, $142.4\left(\mathrm{dd},{ }^{1} J=265 \mathrm{~Hz},{ }^{2} J=21 \mathrm{~Hz}, \mathrm{Ar}-\mathrm{CF}\right), 145.6\left(\mathrm{dd},{ }^{1} J=247 \mathrm{~Hz},{ }^{2} J=22 \mathrm{~Hz}, \mathrm{Ar}-\mathrm{CF}\right)$.

4-Ethoxy-2,3-difluorbenzyltosylhydrazon (142b): Nach AAV 1 wurden $1.00 \mathrm{~g}$ (5.37 mmol) 4-Ethoxy- 2,3-difluorbenzaldehyd mit $1.05 \mathrm{~g} \quad$ (5.64 mmol, 1.05 äq) Tosylhydrazin zur Reaktion gebracht und $2.00 \mathrm{~g}$ (5.26 mmol, 98\%) des Tosylhydrazons isoliert. . - IR (KBr): v[ $\left.\mathrm{cm}^{-1}\right]=3185$ (s), 2982 (d), 2930 (bs), 1623, 1518, 1458, 1334 (s) CF, 1167 (s) CF, 1083 (s) $\mathrm{SO}_{2 .}-{ }^{1} \mathrm{H}-\mathrm{NMR}$ $\left(250 \mathrm{MHz}, \mathrm{C}_{6} \mathrm{D}_{6}\right): \delta=1.45\left(\mathrm{t},{ }^{3} J=6.0 \mathrm{~Hz}, 3 \mathrm{H}, \mathrm{CH}_{3}\right), 2.41\left(\mathrm{~s}, 3 \mathrm{H}, \mathrm{CH}_{3}\right), 4.13$<smiles></smiles>
(q, $\left.{ }^{3} J=6.0 \mathrm{~Hz}, 2 \mathrm{H}, \mathrm{CH}_{2}\right), 6.72\left(\mathrm{t},{ }^{3} J=8.5 \mathrm{~Hz}, 1 \mathrm{H}, \mathrm{Ar}-\mathrm{H}\right), 7.31\left(\mathrm{~d},{ }^{3} J=8.0 \mathrm{~Hz}, 2 \mathrm{H}, \mathrm{Ar}-\mathrm{H}\right)$, $7.51\left(\mathrm{t},{ }^{3} \mathrm{~J}=8.5 \mathrm{~Hz}, 1 \mathrm{H}, \mathrm{Ar}-\mathrm{H}\right) .7 .86\left(\mathrm{~d},{ }^{3} \mathrm{~J}=8.0 \mathrm{~Hz}, 2 \mathrm{H}, \mathrm{Ar}-\mathrm{H}\right), 7.97$ (s, $\left.1 \mathrm{H}, \mathrm{NH}\right)-{ }^{13} \mathrm{C}-$ NMR (62.9 MHz, $\left.\mathrm{CDCl}_{3}, \mathrm{DEPT}\right): \delta=14.6(+) \mathrm{CH}_{3}, 21.5(+) \mathrm{CH}_{3}, 65.4(-) \mathrm{OCH}_{2}, 109.5(+)$

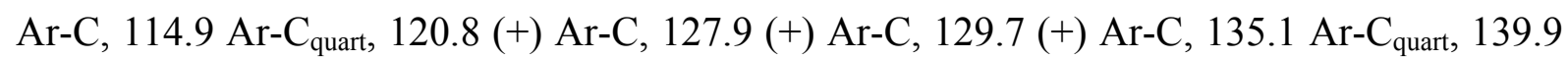
(+) Ar-C, 144.3 Ar-C quart, $158.7(-) \mathrm{C}=\mathrm{N}$.

exo,exo-6-(4-Cyanophenyl)-3-npentylbicyclo[3.1.0]hexan(155a): $\mathrm{Zu}$ einer Lösung von 966 mg (6.99 mmol) 4-nPentylcyclopenten 103 wurden 109 mg (7 Mol\%) Palladiumacetat gegeben und dann eine Lösung von $1.10 \mathrm{~g}$ 4Cyanophenyldiazomethan 143a in $10 \mathrm{ml} \mathrm{Et} 2 \mathrm{O}$ bei $20^{\circ} \mathrm{C}$ während $12 \mathrm{~h}$ zugetropft. Trennung von $600 \mathrm{mg}$ Produktgemisches mittels HPLC (LM Methanol/Wasser, $85: 15)$ ergab $30 \mathrm{mg}$ des endo-Derivates und $180 \mathrm{mg}$ des erwünschten exoDerivates. - ${ }^{1} \mathrm{H}-\mathrm{NMR}\left(250 \mathrm{MHz}, \mathrm{C}_{6} \mathrm{D}_{6}\right): \delta=0.88\left(\mathrm{t},{ }^{3} \mathrm{~J}=6.5 \mathrm{~Hz}, 3 \mathrm{H}, \mathrm{CH}_{3}\right), 1.50$ (m, $14 \mathrm{H}), 2.08\left(\mathrm{dd},{ }^{3} J=12.0,3.0 \mathrm{~Hz}, 2 \mathrm{H}, \mathrm{CH}\right), 7.04$ (d, $\left.{ }^{3} \mathrm{~J}=8.5 \mathrm{~Hz}, 2 \mathrm{H}, \mathrm{Ar}-\mathrm{H}\right)$, (c) $7.48\left(\mathrm{~d},{ }^{3} J=8.5 \mathrm{~Hz}, 2 \mathrm{H}, \mathrm{Ar}-\mathrm{H}\right)-{ }^{13} \mathrm{C}-\mathrm{NMR}\left(62.9 \mathrm{MHz}, \mathrm{CDCl}_{3}, \mathrm{DEPT}\right): \delta=14.0(+) \mathrm{CH}_{3}$, 
$22.5(+) \mathrm{CH}, 22.6(-) \mathrm{CH}_{2}, 24.2(-) \mathrm{CH}_{2}, 28.2(-) \mathrm{CH}_{2}, \quad 31.8(-) \mathrm{CH}_{2}, 32.8$ (-) $\mathrm{CH}_{2}, 36.2$ (+) $\mathrm{CH}, 36.5(-) \mathrm{CH}_{2}, 109.4$ Ar-C $\mathrm{C}_{\text {quart }}, 118.5 \mathrm{CN}, 130.2$ (+) Ar-C, $132.0(+) \mathrm{Ar}-\mathrm{C}, 144.9 \mathrm{Ar}-$ $\mathrm{C}_{\text {quart. }}-\mathrm{MS}(70 \mathrm{eV}, \mathrm{EI}), m / z(\%): 253(100)\left[\mathrm{M}^{+}\right], 182(95)\left[\mathrm{M}^{+}-\mathrm{C}_{5} \mathrm{H}_{11}\right], 168(16), 154(40)$, 142 (65), 137 (35), 127 (15), $116(50)$.

3-\{[4-(trans-4-n-Pentyl)cyclohexyl]phenyl\}bicyclo[3.1.0]hexan-6-

carbonsäure-tertbutylester (162a): Gemäß AAV 5 wurden $4.10 \mathrm{~g}$ (13.3 mmol) Alken 161a und $2.95 \mathrm{~g}(2.87 \mathrm{ml}, 20.8 \mathrm{mmol}) \mathrm{N}_{2} \mathrm{CHCO}_{2} t \mathrm{Bu}$ unter Katalyse von $54.0 \mathrm{mg}(0.07 \mathrm{mmol})\left[\mathrm{Rh}\left(\mathrm{C}_{7} \mathrm{H}_{15} \mathrm{COO}\right)_{2}\right]_{2}$ zur Reaktion gebracht und das Reaktionsprodukt säulenchromatographisch (350 g Kieselgel, Säule $\left.45 \times 4.5 \mathrm{~cm}, \mathrm{Hexan} / \mathrm{Et}_{2} \mathrm{O} 15: 1, R_{\mathrm{f}}=0.45,0.43\right)$ gereinigt. Dabei wurden die beiden Reaktionsprodukte 162a-1 und 162a-2 isoliert.

162a-1: 2.726 g (48\%), farblose Kristalle, Smp. $113-115{ }^{\circ} \mathrm{C}(\mathrm{MeOH}) .-{ }^{1} \mathrm{H}$ NMR: $\delta=0.92\left(\mathrm{t},{ }^{3} J=6.8 \mathrm{~Hz}, 3 \mathrm{H}, \mathrm{CH}_{3}\right), 1.08\left(\mathrm{t},{ }^{3} J=12.0,2 \mathrm{H}, \mathrm{CH}_{2}\right.$ cyclohex.), 1.10-1.31 (m, $10 \mathrm{H}), 1.48$ (s, $9 \mathrm{H}, 3 \mathrm{CH}_{3}$ ), 1.62 (br. s, $1 \mathrm{H}, \mathrm{CH}$

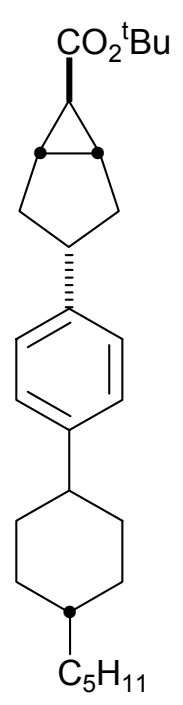
cyclopr.), 1.95-2.05 (m, $9 \mathrm{H}), 2.25$ (dd, ${ }^{3} J=7.3,12.5 \mathrm{~Hz}, 2 \mathrm{H}, \mathrm{CH}_{2}$ cyclopent.), 2.45 (tt, ${ }^{3} J=$ 2.6, $12.3 \mathrm{~Hz}, 1 \mathrm{H}, \mathrm{CH}_{2}$ cyclohex.), 2.70 (tt, ${ }^{3} \mathrm{~J}=6.7,8.9 \mathrm{~Hz}, 1 \mathrm{H}, \mathrm{CH}$ cyclopent.), 7.13 (br. s, $4 \mathrm{H}, \mathrm{Ar}) .-{ }^{13} \mathrm{C}$ NMR: $\delta=28.1\left(3 \mathrm{CH}_{3}\right), 14.1\left(\mathrm{CH}_{3}\right), 33.6,34.3,35.7\left(2 \mathrm{CH}_{2}\right), 22.7,26.6,32.2$, $37.4\left(\mathrm{CH}_{2}\right), 27.8,126.7,127.0(2 \mathrm{CH}), 23.0,37.2,39.6,44.1(\mathrm{CH}), 79.9,141.1,145.7,173.1$ (C).

162a-2: $2.174 \mathrm{~g} \mathrm{(38 \% ),} \mathrm{farblose} \mathrm{Kristalle,} \mathrm{Smp.} 68-70{ }^{\circ} \mathrm{C}(\mathrm{MeOH}) .-{ }^{1} \mathrm{H}$ NMR: $\delta=0.88(\mathrm{t}, J$ $\left.=6.8 \mathrm{~Hz}, 3 \mathrm{H}, \mathrm{CH}_{3}\right), 1.05$ (t, $J=12.0,2 \mathrm{H}, \mathrm{CH}_{2}$ cyclohex.), 1.10-1.31 (m, $\left.9 \mathrm{H}\right), 1.42$ (t, $J=$ $6.5 \mathrm{~Hz}, 2 \mathrm{H}$ ), 1.49 (s, $9 \mathrm{H}, 3 \mathrm{CH}_{3}$ ), 1.60 (br. s, $1 \mathrm{H}, \mathrm{CH}$ cyclopr.), 1.75 (dd, J=2.0, $5.6 \mathrm{~Hz}, 2$ H), $1.85(\mathrm{dm},=9.5 \mathrm{~Hz}, 4 \mathrm{H}), 1.95(\mathrm{tt}, J=2.4,7.2 \mathrm{~Hz}, 2 \mathrm{H}), 2.38(\mathrm{dd}, J=8.0,13.2 \mathrm{~Hz}, 2 \mathrm{H}$, $\mathrm{CH}_{2}$ cyclopent.), 2.40-2.55 (m, $1 \mathrm{H}$.), 2.89 (tt, $J=6.5,8.0 \mathrm{~Hz}, 1 \mathrm{H}, \mathrm{CH}$ cyclopent.), 7.11 (br. s, $4 \mathrm{H}, \mathrm{Ar}) .-{ }^{13} \mathrm{C} \mathrm{NMR}: \delta=27.9\left(3 \mathrm{CH}_{3}\right), 14.1\left(\mathrm{CH}_{3}\right), 33.6,34.3,35.0\left(2 \mathrm{CH}_{2}\right), 22.6,26.6$, 32.1, 37.4 $\left(\mathrm{CH}_{2}\right), 28.1,126.7,127.0(2 \mathrm{CH}), 23.5,37.3,41.8,44.1(\mathrm{CH}), 80.2,143.0,145.4$, 170.7 (C). Es wurden zur Stukturbestimmung von beiden Produkten Kristalle $\left(\mathrm{MeOH} / \mathrm{Et}_{2} \mathrm{O}, 0\right.$ ${ }^{\circ} \mathrm{C}$ ) angefertigt. Es konnte nur von dem Diastereomer 162a-2 eine Röntgenstruktur erhalten werden. Diese belegte exo,endo-Konfiguration. Aus diesem Ergebnis wurde geschlossen, dass 162a-1 die erwünschte exo,exo-Konfiguration aufweisen muss. 
exo,exo-3-(3,4,5-Trifluorophenyl)bicyclo[3.1.0]hexan-6-carbonsäure-

tertbutylester (162b): Nach AAV 5 wurden 4.99 g (25.2 mmol) Alken 161b mit $5.37 \mathrm{~g}(5.24 \mathrm{ml}, 37.8 \mathrm{mmol}) \mathrm{N}_{2} \mathrm{CHCO}_{2} t \mathrm{Bu}$ unter Katalyse von $98 \mathrm{mg}(0.13 \mathrm{mmol})\left[\mathrm{Rh}\left(\mathrm{C}_{7} \mathrm{H}_{15} \mathrm{COO}\right)_{2}\right]_{2}$ zur Reaktion gebracht und nach säulenchromatographischer $(350 \mathrm{~g}$ Kieselgel, Säule $45 \times 4.5 \mathrm{~cm}$, Hexan/Et $\mathrm{E}_{2} \mathrm{O} 20: 1, R_{\mathrm{f}}=0.31$.) Reinigung konnten $4.87 \mathrm{~g}(62 \%)$ zweier chromatographisch nicht weiter trennbarer Diastereomere 162b (3:1) isoliert werden. Das Hauptprodukt ist gemäß ${ }^{1} \mathrm{H}-\mathrm{NMR}-$ Spektrum mit 47\%

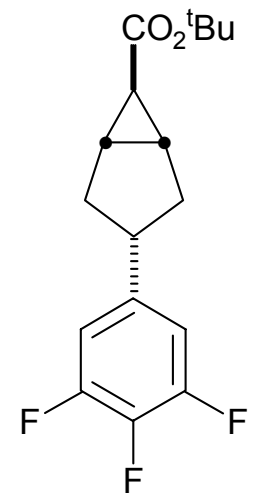
gebildet worden. Durch Kristallisation aus Methanol konnten $2.46 \mathrm{~g}$ (31\%) der Hauptkomponente isoliert werden. Durch Einengen der Mutterlauge und erneuter Kristallisation aus Methanol bei $-20^{\circ} \mathrm{C}$ konnten weitere $610 \mathrm{mg}$ des Zielprodukts mit einem Schmelzpunkt von Smp. $88{ }^{\circ} \mathrm{C}$ isoliert werden. Es konnten insgesamt $3.07 \mathrm{~g}$ (39\%) der Titelverbindung 162b-1 isoliert werden..

162b-1: - IR (KBr): $v\left[\mathrm{~cm}^{-1}\right]=3396,3051,2957,2923,2861,1709(\mathrm{C}=\mathrm{O}), 1622,1533$, 1404, 1368, 1321, 1151 (Ar-F), 1036, 832, 692. - ${ }^{1} \mathrm{H}$ NMR: $\delta=1.44$ (s, 9 H, $3 \mathrm{CH}_{3}$ ), 1.54 (t, $J=2,3 \mathrm{~Hz}, 1 \mathrm{H}, \mathrm{CH}$ cycloprop.), 1.80 (dd, $\left.J=3.0,12.3 \mathrm{~Hz}, 2 \mathrm{H}, \mathrm{CH}_{2}\right), 1.90$ (m, $2 \mathrm{H}, 2 \mathrm{CH}$ cycloprop.), 2.23 (dd, $J=7.3,12.3 \mathrm{~Hz}, 2 \mathrm{H}, \mathrm{CH}_{2}$ ), 2.63 (tt, $J=7.3,10.4 \mathrm{~Hz}, 1 \mathrm{H}, \mathrm{CH}$ ), 6.76 (dd, $J=6.5,9.0 \mathrm{~Hz}, 2 \mathrm{H}, \mathrm{Ar}) .-{ }^{13} \mathrm{C}$ NMR: $\delta=28.1\left(3 \mathrm{CH}_{3}\right), 35.5\left(2 \mathrm{CH}_{2}\right), 27.3,111.0$ (ddd, $J$ $=6.8,13.6,20.4 \mathrm{~Hz})(2 \mathrm{CH}), 23.0,39.5(\mathrm{CH}), 151.1$ (ddd, $J=4.5,9.6,250.0 \mathrm{~Hz})(2 \mathrm{C})$, 80.27, 138.1 (dt, $J=15.4,264.2 \mathrm{~Hz}$ ), 140.3 (dt, $J=4.6,6.7 \mathrm{~Hz}), 172.7$ (C). $-\mathrm{C}_{17} \mathrm{H}_{19} \mathrm{~F}_{3} \mathrm{O}_{2}$ (312.32): ber. C 65.37, H 6.13\%; gem. C 65.22, H 6.09.

Die Konfiguration der Substanz im Festkörper wurde durch Röntgenstrukturanalyse (Kristalle aus $\mathrm{MeOH} / \mathrm{Et}_{2} \mathrm{O}, 0{ }^{\circ} \mathrm{C}$ ) als exo,exo festgestellt.

exo,exo-3-[4-(3,4,5-Trifluorophenyl)phenyl]bicyclo[3.1.0] hexan-6carbonsäuretertbutylester (162c): Gemäss $\quad$ AAV 5 wurden $4.07 \mathrm{~g}$ (16.5 mmol) Alken und $3.51 \mathrm{~g}$ (3.42 ml, $24.7 \mathrm{mmol}) \mathrm{N}_{2} \mathrm{CHCO}_{2} t \mathrm{Bu}$ unter Katalyse von $64 \mathrm{mg}(0.08 \mathrm{mmol})\left[\mathrm{Rh}\left(\mathrm{C}_{7} \mathrm{H}_{15} \mathrm{COO}\right)_{2}\right]_{2}$ zur Reaktion gebracht und nach säulenchromatographischer $(450 \mathrm{~g}$ Kieselgel, Säule $40 \times 5.6 \mathrm{~cm}$, Hexan/ $\left.\mathrm{Et}_{2} \mathrm{O} 10: 1, R_{\mathrm{f}}=0.30\right)$ Aufarbeitung zwei chromatographisch nicht weiter trennbare Diastereomere isoliert. Feinkristallisation aus $\mathrm{MeOH} / \mathrm{CHCl}_{3}$ (15:1) ergab $2.07 \mathrm{~g}$ (32\%) des Zielprodukts 162c als farblose Kristalle mit dem Sdp. $150-151^{\circ} \mathrm{C}$. Einengen der Mutterlauge und erneute

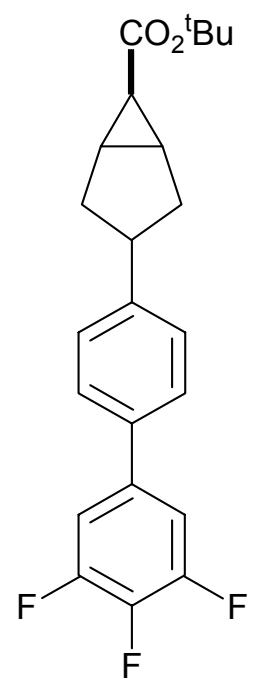


Kristallisation ergaben zusätzliche $606 \mathrm{mg}$ der Titelverbindung 162c. Gesamtausbeute: 42\%. $-{ }^{1} \mathrm{H}$ NMR: $\delta=1.47$ (s, $9 \mathrm{H}, 3 \mathrm{CH}_{3}$ ), 1.62 (t, $J=2,2 \mathrm{~Hz}, 1 \mathrm{H}, \mathrm{CH}$ cycloprop.), 1.85-2.08 (m, 4 $\mathrm{H}, 2 \mathrm{CH}$ cycloprop $\left.+\mathrm{CH}_{2}\right), 2.28\left(\mathrm{dd}, J=7.3,12.5 \mathrm{~Hz}, 2 \mathrm{H}, \mathrm{CH}_{2}\right), 2.75(\mathrm{tt}, J=7.3,10.3 \mathrm{~Hz}, 1$ $\mathrm{H}, \mathrm{CH}), 7.17$ (dd, $J=6.5,9.0 \mathrm{~Hz}, 2 \mathrm{H}, \mathrm{Ar}), 7.27$ (d, $J=8.3 \mathrm{~Hz}, 2 \mathrm{H}, \mathrm{Ar}), 7.41$ (d, $J=8.3 \mathrm{~Hz}$, $2 \mathrm{H}, \mathrm{Ar}) .-{ }^{13} \mathrm{C}$ NMR: $\delta=28.2\left(3 \mathrm{CH}_{3}\right), 35.7\left(2 \mathrm{CH}_{2}\right), 27.7,111.7(\mathrm{ddd}, J=7.1,14.3,21.3$ Hz) 126.8, 127.9 (2 CH), 23.1, $39.7(\mathrm{CH}), 151.3$ (ddd, $J=4.5,10.3,259.1 \mathrm{~Hz})(2 \mathrm{C}), 80.27$, 136.1, 137.1 (dt, $J=4.6,6.7 \mathrm{~Hz}), 139.1$ (dt, $J=23.8,251.5 \mathrm{~Hz}), 144.3,173.0$ (C). $\mathrm{C}_{23} \mathrm{H}_{23} \mathrm{~F}_{3} \mathrm{O}_{2}$ (388.41): ber. C 71.12, H 5.97\%; gem. C 70.88, H 6.03\%.

exo,exo-3-[\{trans-4-[(trans-4-n-Propyl)cyclohexyl]cyclohexyl\}phenyl]bicyclo[3.1.0] hexan-6-carbonsäuretertbutylester (162d): Gemäss AAV 5 wurden $4.31 \mathrm{~g}$ (12.3 mmol) Alken und $2.61 \mathrm{~g}$ ( $2.55 \mathrm{ml}, 18.4 \mathrm{mmol}) \mathrm{N}_{2} \mathrm{CHCO}_{2} t \mathrm{Bu}$ unter Katalyse von $49 \mathrm{mg}(0.06 \mathrm{mmol}) \quad\left[\mathrm{Rh}\left(\mathrm{C}_{7} \mathrm{H}_{15} \mathrm{COO}\right)_{2}\right]_{2}$ umgesetzt und nach säulenchromatographischer (350 g Kieselgel, Säule $45 \times 4.5 \mathrm{~cm}, \mathrm{Hexan} / \mathrm{Et}_{2} \mathrm{O}$ 15:1, $R_{\mathrm{f}}=0.44$ und 0.39) Aufarbeitung zwei Diastereomere und $889 \mathrm{mg}$ Alken $\left(R_{\mathrm{f}}=0.73\right)$ erhalten. Die Diastereomere konnten durch Feinkristallisation aus $\mathrm{MeOH} / \mathrm{CHCl}_{3}$ (10:1) weiter gereinigt werden. Es wurden $1.52 \mathrm{~g}$ (27\%) exo,endo-Derivat und $2.68 \mathrm{~g}(47 \%)$ exo,exo-Derivat isoliert.

exo,exo-162d: farblose Kristalle, Smp. 281-283 ${ }^{\circ} \mathrm{C}$ (Zers.). - IR (KBr): v[ $\left.\mathrm{cm}^{-1}\right]=3048,3007,2927,2846,1709(\mathrm{C}=\mathrm{O}), 1450,1410,1157,1062,840,557$. $-{ }^{1} \mathrm{H}$ NMR: $\delta=0.88\left(\mathrm{t},{ }^{3} J=7.1 \mathrm{~Hz}, 3 \mathrm{H}, \mathrm{CH}_{3}\right), 0.97-1.18(\mathrm{~m}, 8 \mathrm{H}), 1.27-1.42$ (m, $2 \mathrm{H}), 1.45$ (s, $9 \mathrm{H}, 3 \mathrm{CH}_{3}$ ), 1.63 (br. s, $1 \mathrm{H}, \mathrm{CH}$ cyclopr.), 1.73-1.97 (m, 16 (1) H), 2.23 (dd, ${ }^{3} J=7.0,12.3 \mathrm{~Hz}, 2 \mathrm{H}, \mathrm{CH}_{2}$ cyclopent.), 2.41 (tt, ${ }^{3} \mathrm{~J}=2.6,12.0 \mathrm{~Hz}, 2 \mathrm{H}, \mathrm{CH}_{2}$ cyclohex.), 2.67 (tt, ${ }^{3} J=7.0,9.4 \mathrm{~Hz}, 1 \mathrm{H}, \mathrm{CH}$ cyclopent.), 7.11 (br. s, $4 \mathrm{H}, \mathrm{Ar}$ ). $-{ }^{13} \mathrm{C} \mathrm{NMR}: \delta$ $=28.2\left(3 \mathrm{CH}_{3}\right), 14.4\left(\mathrm{CH}_{3}\right), 30.1,10.3,33.6,34.6,35.8\left(2 \mathrm{CH}_{2}\right), 20.0,39.8\left(\mathrm{CH}_{2}\right), 27.8$, 126.7, $127.1(2 \mathrm{CH}), 23.0,37.6,39.6,42.9,43.4,44.2(\mathrm{CH}), 80.0,141.1,145.8,173.3(\mathrm{C}) .-$ $\mathrm{C}_{32} \mathrm{H}_{48} \mathrm{O}_{2}$ (464.7): ber. C 82.70, H 10.41\%; gem. C 82.53, H 10.19\%. 
exo,exo-(3-\{[4-(trans-4-n-Pentyl)cyclohexyl]phenyl\}bicyclo[3.1.0]hex-6-

yl)methanol (163a): Gemäss AAV6 wurden $2.17 \mathrm{~g}$ (5.29 mmol) tert-Butylester 162a, mit $\mathrm{LiAlH}_{4}$ zur Reaktion gebracht und 1.80 g (quant.) des Alkohols 163a (Smp. $\left.141^{\circ} \mathrm{C}\right)$ als farbloser Feststoff (Hexan) isoliert. - IR $(\mathrm{KBr}): v\left[\mathrm{~cm}^{-1}\right]=$ 3325, 3017, 2957, 2931, 2851, 1899, 1514, 1465, 1443, 1415, 1368, 1289, 1264, 1237, 1208, 1117, 1062, 1022, 997, 943, 895, 827, 768, 725, 663, 556. - ${ }^{1} \mathrm{H}$ NMR: $\delta=0.91\left(\mathrm{t},{ }^{3} J=6.9 \mathrm{~Hz}, 3 \mathrm{H}, \mathrm{CH}_{3}\right), 1.04\left(\mathrm{qd},{ }^{3} J=3.0,12.3 \mathrm{~Hz}, 2 \mathrm{H}\right)$, $1.17-1.34(\mathrm{~m}, 8 \mathrm{H}), 1.40(\mathrm{pd},=3.0,13.5 \mathrm{~Hz}, 4 \mathrm{H}), 1.73\left(\mathrm{dd},{ }^{3} J=7.8,13.5 \mathrm{~Hz}, 1\right.$ H), 1.83-1.91 (m, $7 \mathrm{H}), 2.19$ (dd, $\left.{ }^{3} J=7.5,12.5 \mathrm{~Hz}, 2 \mathrm{H}, \mathrm{CH}_{2}\right), 2.43$ (tq, ${ }^{3} J=3.3$, $12.5 \mathrm{~Hz}, 2 \mathrm{H}), 2.72\left(\mathrm{tt},{ }^{3} J=7.4,10.1 \mathrm{~Hz}, 1 \mathrm{H}, \mathrm{CH}\right), 3.46\left(\mathrm{~d},{ }^{3} J=7.0 \mathrm{~Hz}, 2 \mathrm{H}\right.$,

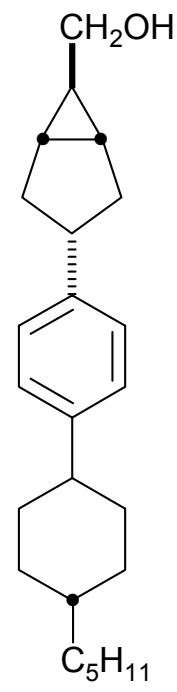
$\left.\mathrm{CH}_{2} \mathrm{O}\right), 7.13$ (br. s, $\left.4 \mathrm{H}, \mathrm{Ar}\right) .-{ }^{13} \mathrm{C}$ NMR: $\delta=14.1\left(\mathrm{CH}_{3}\right), 33.6,34.4,35.8\left(2 \mathrm{CH}_{2}\right), 22.7,26.7$, 32.2, 37.4, $66.0\left(\mathrm{CH}_{2}\right), 22.3,126.7,127.1(2 \mathrm{CH}), 22.4,37.3,40.3,44.1(\mathrm{CH}), 141.7,145.6$ (C). $-\mathrm{C}_{24} \mathrm{H}_{36} \mathrm{O}$ (340.53): ber. C 84.64, H 10.66\%; gem. C 84.90, H 10.55\%.

exo,exo-\{3-(3,4,5-Trifluorophenyl)bicyclo[3.1.0] hex-6-yl\}methanol (163b): Gemäss AAV 6 wurden $2.42 \mathrm{~g}(7.74 \mathrm{mmol})$ des Esters 162b mit $\mathrm{LiAlH}_{4}$ umgesetzt, $1.87 \mathrm{~g}$ (quant.) des Alkohols 163b wurden als farbloser Feststoff (Smp. $40{ }^{\circ} \mathrm{C}$ ) isoliert. $-{ }^{1} \mathrm{H}$ NMR: $\delta=1.09\left(\mathrm{tt},{ }^{3} J=3.3,7.0 \mathrm{~Hz}, 1\right.$ H, CH cycloprop.), 1.21-1.31 (m, 2 H, 2 CH cycloprop.), 1.73 (ddd, ${ }^{3} J=$ 3.8, 11.0, $\left.12.5 \mathrm{~Hz}, 2 \mathrm{H}, \mathrm{CH}_{2}\right), 2.14\left(\mathrm{dd},{ }^{3} J=7.5,12.5 \mathrm{~Hz}, 2 \mathrm{H}, \mathrm{CH}_{2}\right), 2.44$ (s, $1 \mathrm{H}, \mathrm{OH}), 2.63\left(\mathrm{tt},{ }^{3} J=7.5,11.0 \mathrm{~Hz}, 1 \mathrm{H}, \mathrm{CH}\right), 3.40\left(\mathrm{~d},{ }^{3} J=7.0 \mathrm{~Hz}, 2 \mathrm{H}\right.$, $\left.\mathrm{CH}_{2} \mathrm{O}\right), 6.73\left(\mathrm{dd},{ }^{3} J=6.8,9.0 \mathrm{~Hz}, 2 \mathrm{H}, \mathrm{Ar}\right) .-{ }^{13} \mathrm{C}$ NMR: $\delta=35.4\left(2 \mathrm{CH}_{2}\right)$,

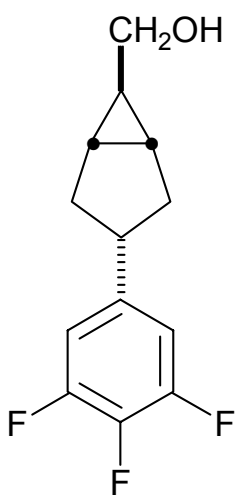
$65.3\left(\mathrm{CH}_{2}\right), 21.9,110.9$ (ddd, $\left.J=6.7,13.8,20.4 \mathrm{~Hz}\right)(2 \mathrm{CH}), 22.1,40.0(\mathrm{CH}), 150.8$ (ddd, $J=$ 4.1, 9.8, 248.7 Hz) (2 C), $137.7(\mathrm{dt}, J=15.4,250.0 \mathrm{~Hz}), 140.3(\mathrm{dt}, J=6.9,11.3 \mathrm{~Hz})(\mathrm{C})$. 
exo,exo-\{3-[4-(3,4,5-Trifluorophenyl)phenyl]bicyclo[3.1.0]hex-6-

yl;methanol (163c): Gemäss AAV 6 wurden $2.45 \mathrm{~g}$, (6.30 mmol) des Esters 162c mit $\mathrm{LiAlH}_{4}$ umgesetzt und $2.00 \mathrm{~g}$ (quant.) des Alkohols 163c als farbloser Feststoff (Smp. $\left.78-80^{\circ} \mathrm{C}\right)$ isoliert. - IR $(\mathrm{KBr}): v\left[\mathrm{~cm}^{-1}\right]=$ 3300, 3087, 3025, 2995, 2945, 2930, 2859, 1903, 1793, 1615, 1539, 1510, 1443, 1403, 1363, 1250, 1120, 1048, 946, 866, 828, 767, 702, 539. $-{ }^{1} \mathrm{H}$ NMR: $\delta=1.22\left(\mathrm{tt},{ }^{3} J=3.3,7.0 \mathrm{~Hz}, 1 \mathrm{H}, \mathrm{CH}\right.$ cycloprop.), 1.35 (m, 2 H, 2 CH cycloprop.), 1.89 (ddd, ${ }^{3} J=2.8,11.0,12.8 \mathrm{~Hz}, 2 \mathrm{H}, \mathrm{CH}_{2}$ ), 2.06 (s, $1 \mathrm{H}, \mathrm{OH}), 2.22\left(\mathrm{dd},{ }^{3} J=7.5,12.8 \mathrm{~Hz}, 2 \mathrm{H}, \mathrm{CH}_{2}\right), 2.79\left(\mathrm{tt},{ }^{3} \mathrm{~J}=7.5\right.$, $11.0 \mathrm{~Hz}, 1 \mathrm{H}, \mathrm{CH}), 3.48\left(\mathrm{~d},{ }^{3} J=7.0 \mathrm{~Hz}, 2 \mathrm{H}, \mathrm{CH}_{2} \mathrm{O}\right), 7.14\left(\mathrm{dd},{ }^{3} J=6.5\right.$,

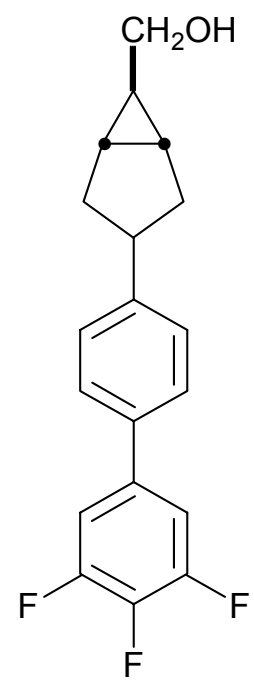
$8.8 \mathrm{~Hz}, 2 \mathrm{H}, \mathrm{Ar}), 7.27\left(\mathrm{~d},{ }^{3} J=8.3 \mathrm{~Hz}, 2 \mathrm{H}, \mathrm{Ar}\right), 7.40\left(\mathrm{~d},{ }^{3} J=8.3 \mathrm{~Hz}, 2 \mathrm{H}\right.$, Ar). $-{ }^{13} \mathrm{C}$ NMR: $\delta=35.7\left(2 \mathrm{CH}_{2}\right), 65.7\left(\mathrm{CH}_{2}\right), 22.2,110.6(\mathrm{ddd}, J=7.1,14.1,21.3 \mathrm{~Hz})$, 126.6, 127.9 (2 CH), 22.3, $40.3(\mathrm{CH}), 151.3$ (ddd, $J=4.4,10.1,249.2 \mathrm{~Hz})(2 \mathrm{C}), 135.8,137.1$ (dt, $J=4.6,7.8 \mathrm{~Hz}) 138.9$ (dt, $J=15.4,251.4 \mathrm{~Hz}), 144.9$ (C). $-\mathrm{C}_{19} \mathrm{H}_{17} \mathrm{~F}_{3} \mathrm{O}$ (318.33): ber. C 71.68, H 5.38\%; gem. C 71.51, H 5.11\%.

exo,exo-(3-[\{trans-4-[(trans-4-n-

Propyl)cyclohexyl]cyclohexyl\}phenyl]bicyclo-[3.1.0]hex-6-yl)methanol

(163d): Gemäß AAV 6 wurden $2.68 \mathrm{~g}(5.76 \mathrm{mmol})$ des Esters 162d mit $\mathrm{LiAlH}_{4}$ umgesetzt und $2.26 \mathrm{~g}$ (quant.) des Alkohols 163d als farbloser Feststoff (Smp. 259-260 ${ }^{\circ} \mathrm{C}$ (Zers.) (Hexan)) isoliert. - IR (KBr): $v\left[\mathrm{~cm}^{-1}\right]=3325,3018,2958$, $2847,1515,1445,1119,1062,1020,943,895,824,559-{ }^{1} \mathrm{H}$ NMR: $\delta=0.78$ (m, $1 \mathrm{H}, \mathrm{CH}$ cycloprop.), 0.89 ( $\left.\mathrm{t},{ }^{3} J=7.1 \mathrm{~Hz}, 3 \mathrm{H}, \mathrm{CH}_{3}\right), 1.03-1.61$ (m, $8 \mathrm{H}$ ), 1.30-1.57 (m, $6 \mathrm{H}), 1.73\left(\mathrm{dd},{ }^{3} J=7.8,13.5 \mathrm{~Hz}, 1 \mathrm{H}\right), 1.68$ (s, $\left.1 \mathrm{H}, \mathrm{OH}\right), 1.73-$ $1.95(\mathrm{~m}, 12 \mathrm{H}), 2.19\left(\mathrm{dd},{ }^{3} J=7.5,12.5 \mathrm{~Hz}, 2 \mathrm{H}, \mathrm{CH}_{2}\right), 2.42\left(\mathrm{tq},{ }^{3} J=3.3,11.9\right.$ $\mathrm{Hz}, 2 \mathrm{H}), 2.72\left(\mathrm{tt},{ }^{3} J=7.5,11.3 \mathrm{~Hz}, 1 \mathrm{H}, \mathrm{CH}\right), 3.46\left(\mathrm{~d},{ }^{3} J=7.3 \mathrm{~Hz}, 2 \mathrm{H}\right.$, $\mathrm{CH}_{2} \mathrm{O}$ ), 7.13 (br. s, $\left.4 \mathrm{H}, \mathrm{Ar}\right) .-{ }^{13} \mathrm{C}$ NMR: $\delta=14.4\left(\mathrm{CH}_{3}\right), 30.0,30.3,33.6$, 34.6, $35.8\left(2 \mathrm{CH}_{2}\right), 20.0,39.8,66.0\left(\mathrm{CH}_{2}\right), 22.2,126.7,127.1(2 \mathrm{CH}), 22.3$, 37.6, 40.3, 42.8, 43.4, 44.1 (CH), 141.7, $145.6(\mathrm{C}) .-\mathrm{C}_{28} \mathrm{H}_{42} \mathrm{O}$ (394.62): ber C (O) 85.22, H 10.73\%; gem. C 85.04, H 10.48\%. 
exo,exo-6-(Jodomethyl)-3-\{[4-(trans-4-n-

pentyl)cyclohexyl]phenyl\}bicyclo[3.1.0]hexan (164a): Gemäss AAV7 wurden $1.76 \mathrm{~g}$ (5.17 mmol) des Alkohols 163a mit $482 \mathrm{mg}$ (7.08 mmol) Im-H und $1.76 \mathrm{~g}$ (6.72 mmol) $\mathrm{Ph}_{3} \mathrm{P}$ und $1.97 \mathrm{~g}(7.75 \mathrm{mmol})$ Jod umgesetzt und nach säulenchromatographischer Aufarbeitung (50 g Kieselgel, Säule $20 \times 3 \mathrm{~cm}$, Hexan/Et $\left.{ }_{2} \mathrm{O} 5: 1, R_{\mathrm{f}}=0.54\right) 2.28 \mathrm{~g}(98 \%)$ des Jodids 164a als farblose Kristalle (Smp. 96-97 ${ }^{\circ} \mathrm{C}$ (Hexan-MeOH) isoliert. $-{ }^{1} \mathrm{H}$ NMR: $\delta=0.9\left(\mathrm{t},{ }^{3} J=6.6 \mathrm{~Hz}, 3\right.$ $\left.\mathrm{H}, \mathrm{CH}_{3}\right), 1.05\left(\mathrm{t},{ }^{3} \mathrm{~J}=11.3 \mathrm{~Hz}, 2 \mathrm{H}\right), 1.18-1.45(\mathrm{~m}, 15 \mathrm{H}), 1.71-1.81(\mathrm{~m}, 1 \mathrm{H}$, $\mathrm{CH}), 1.85\left(\mathrm{dd},{ }^{3} \mathrm{~J}=8.0,8.5 \mathrm{~Hz}, 4 \mathrm{H}, 2 \mathrm{CH}_{2}\right), 2.17\left(\mathrm{dd},{ }^{3} J=7.5,12.5 \mathrm{~Hz}, 2 \mathrm{H}\right.$, $\left.\mathrm{CH}_{2}\right), 2.42\left(\mathrm{tt},{ }^{3} J=3.1,10.5 \mathrm{~Hz}, 1 \mathrm{H}, \mathrm{CH}\right), 2.70\left(\mathrm{tt},{ }^{3} J=7.5,11.2 \mathrm{~Hz}, 1 \mathrm{H}\right.$, $\mathrm{CH}), 3.14\left(\mathrm{~d},{ }^{3} J=7.5 \mathrm{~Hz}, 2 \mathrm{H}, \mathrm{CH}_{2} \mathrm{I}\right), 7.09$ (d, $\left.{ }^{3} J=7.5 \mathrm{~Hz}, 2 \mathrm{H}, \mathrm{Ar}\right), 7.13\left(\mathrm{~d},{ }^{3} J=7.5 \mathrm{~Hz}, 2 \mathrm{H}\right.$, Ar). $-{ }^{13} \mathrm{C}$ NMR: $\delta=12.4,14.1\left(\mathrm{CH}_{3}\right), 22.7,24.5,26.6,30.6,32.2,33.6,34.4,36.2\left(2 \mathrm{CH}_{2}\right)$, 37.3, $37.4\left(\mathrm{CH}_{2}\right), 40.2$, $44.1(\mathrm{CH}), 126.7,127.1(2 \mathrm{CH}), 141.4,145.7\left(\mathrm{C}_{\text {quart }}\right) .-\mathrm{MS}(\mathrm{EI}), \mathrm{m} / \mathrm{z}$ (\%): $450(8)[\mathrm{M}]^{+}, 323(100)[\mathrm{M}-\mathrm{I}]^{+}$. - MS (HR-EI): $450.1783\left(\mathrm{C}_{24} \mathrm{H}_{35} \mathrm{I}\right.$, ber. 450.17834).

exo,exo-6-(Jodomethyl)-3-(3,4,5-trifluorophenyl)bicyclo[3.1.0]hexan (164b): Gemäss AAV 7 wurden $1.88 \mathrm{~g}$ (7.74 mmol) des Alkohols 163b, $723 \mathrm{mg}$ (10.6 mmol) Im-H, $2.63 \mathrm{~g}$ (10.1 mmol) $\mathrm{Ph}_{3} \mathrm{P}$ und $2.95 \mathrm{~g}$ Jod (11.6 mmol) umgesetzt und nach säulenchromatographischer Aufarbeitung (80 g Kieselgel, Säule $30 \times 3 \mathrm{~cm}$, Hexan/Et $\left.{ }_{2} \mathrm{O} 5: 1, R_{\mathrm{f}}=0.60\right) 2.66 \mathrm{~g}(98 \%)$ der Zielverbindung 164b als farbloses Ö1 isoliert. $-{ }^{1} \mathrm{H}$ NMR: $\delta=1.32$ (tt, ${ }^{3} J=3.0,7.5 \mathrm{~Hz}, 1 \mathrm{H}, \mathrm{CH}$ cycloprop.), 1.33-1.36 (m, $2 \mathrm{H}, 2 \mathrm{CH}$ cycloprop.), 1.72 (ddd, ${ }^{3} J=3.0,11.0,12.8 \mathrm{~Hz}, 2 \mathrm{H}, \mathrm{CH}_{2}$ ), 2.17 (dd, ${ }^{3} J=$

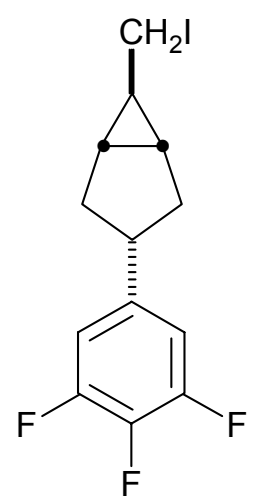
7.5, $\left.12.8 \mathrm{~Hz}, 2 \mathrm{H}, \mathrm{CH}_{2}\right), 2.65\left(\mathrm{tt},{ }^{3} J=7.5,11.0 \mathrm{~Hz}, 1 \mathrm{H}, \mathrm{CH}\right), 3.11\left(\mathrm{~d},{ }^{3} J=7.5 \mathrm{~Hz}, 2 \mathrm{H}, \mathrm{CH}_{2} \mathrm{I}\right)$, $6.77\left(\mathrm{ddd},{ }^{3} J=3.2,4.0,8.8 \mathrm{~Hz}, 2 \mathrm{H}, \mathrm{Ar}\right) .-{ }^{13} \mathrm{C}$ NMR: $\delta=35.8\left(2 \mathrm{CH}_{2}\right), 11.5\left(\mathrm{CH}_{2}\right), 30.1$, $111.0\left(\mathrm{ddd},{ }^{3} J=6.6,13.7,20.4 \mathrm{~Hz}\right)(2 \mathrm{CH}), 24.4,39.9(\mathrm{CH}), 150.9\left(\mathrm{ddd},{ }^{3} J=4.3,9.9,249.0\right.$ Hz) (2 C), 137.9 (dt, $J=15.4,249.1 \mathrm{~Hz}), 140.4$ (dt, $J=4.5,11.3 \mathrm{~Hz})(\mathrm{C}) .-\mathrm{MS}(\mathrm{EI}), m / z(\%)$ : $352(10)[\mathrm{M}]^{+}, 225$ (100) [M - I] $]^{+}$- MS (HR-EI): $351.9936\left(\mathrm{C}_{13} \mathrm{H}_{12} \mathrm{~F}_{3} \mathrm{I}\right.$, ber. 450.17834). $\mathrm{C}_{13} \mathrm{H}_{12} \mathrm{~F}_{3} \mathrm{I}$ (352.13): ber. C 43.50, H 3.44, I 35.94\%; gem. C 43.36, H 3.50. 
exo,exo-6-(Jodomethyl)-3-[4-(3,4,5-

trifluorophenyl)phenyl]bicyclo[3.1.0]hexan (164c): Gemäss AAV 7 wurden $2.00 \mathrm{~g}$ (6.29 mmol) des Alkohols 163c, $508 \mathrm{mg}$ (7.46 mmol) Im-H, $1.86 \mathrm{~g}$ (7.08 mmol) $\mathrm{Ph}_{3} \mathrm{P}$ und $2.19 \mathrm{~g}(8.62 \mathrm{mmol})$ Jod umgesetzt und nach säulenchromatographischer Aufarbeitung (100 g Kieselgel, Säule $35 \times 3 \mathrm{~cm}$, Hexan/Et $\left.\mathrm{E}_{2} \mathrm{O} 10: 1, R_{\mathrm{f}}=0.55\right) 2.44 \mathrm{~g}(91 \%)$ der Titelverbindung $164 \mathrm{c}$ als farbloses Öl, das beim Stehen bei $0{ }^{\circ} \mathrm{C}$ über Nacht erstarrte (Smp. 60-61 $\left.{ }^{\circ} \mathrm{C}\right) .-{ }^{1} \mathrm{H}$ NMR: $\delta=1.29$ (tt, ${ }^{3} J=3.0,7.5 \mathrm{~Hz}, 1 \mathrm{H}, \mathrm{CH}$ cycloprop.), $1.35-$ 1.47 (m, 2 H, 2 CH cycloprop.), 1.86 (ddd, ${ }^{3} J=3.0,10.8,12.8 \mathrm{~Hz}, 2 \mathrm{H}$, $\left.\mathrm{CH}_{2}\right), 2.24\left(\mathrm{dd},{ }^{3 J}=7.5,12.8 \mathrm{~Hz}, 2 \mathrm{H}, \mathrm{CH}_{2}\right), 2.79\left(\mathrm{tt},{ }^{3} J=7.5,10.8 \mathrm{~Hz}, 1 \mathrm{H}\right.$,

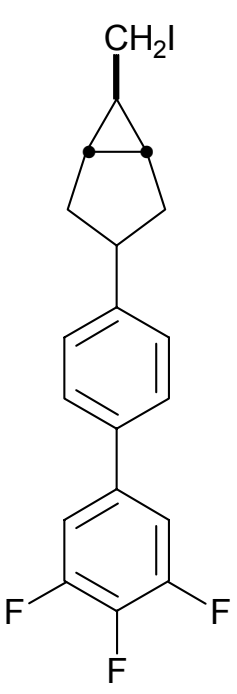
$\mathrm{CH}), 3.17\left(\mathrm{~d},{ }^{3} \mathrm{~J}=7.5 \mathrm{~Hz}, 2 \mathrm{H}, \mathrm{CH}_{2} \mathrm{I}\right), 7.17\left(\mathrm{ddd},{ }^{3} \mathrm{~J}=0.5,7.2,8.8 \mathrm{~Hz}, 2 \mathrm{H}\right.$, Ar), $7.28\left(\mathrm{~d},{ }^{3} J=8.3 \mathrm{~Hz}, 2 \mathrm{H}, \mathrm{Ar}\right), 7.37\left(\mathrm{~d},{ }^{3} J=8.3 \mathrm{~Hz}, 2 \mathrm{H}, \mathrm{Ar}\right) .-{ }^{13} \mathrm{C}$ NMR: $\delta=36.1(2$ $\left.\mathrm{CH}_{2}\right), 12.1\left(\mathrm{CH}_{2}\right), 30.5,111.7(\mathrm{dd}, J=18.3,32.0 \mathrm{~Hz}), 126.6,127.9(2 \mathrm{CH}), 24.4,40.1(\mathrm{CH})$, 151.2 (ddd, $J=4.3,10.1,249.3 \mathrm{~Hz})(2 \mathrm{C}), 135.9,136.9$ (dt, $J=4.5,15.0 \mathrm{~Hz}), 139.0$ (dt, $J=$ 15.4, $251.9 \mathrm{~Hz}), 144.6(\mathrm{C}) .-\mathrm{C}_{19} \mathrm{H}_{16} \mathrm{~F}_{3} \mathrm{I}$ (428.22): ber. C 53.29, H 3.77, I 29.63\%; gem. C 52.69, H 3.80 .

exo,exo-6-(Jodomethyl)-3-[\{trans-4-[(trans-4-n-

propyl)cyclohexyl]cyclohexyl\}phenyl]- bicyclo[3.1.0]hexan (164d): Gemäss AAV 7 wurden $1.87 \mathrm{~g}$ (4.37 mmol) des Alkohols 163d, $442 \mathrm{mg}(6.49 \mathrm{mmol})$ Im-H, $1.61 \mathrm{~g}$ (6.15 mmol) $\mathrm{Ph}_{3} \mathrm{P}$ und $1.80 \mathrm{~g}(7.10 \mathrm{mmol})$ Jod umgesetzt und nach säulenchromatographischer Aufarbeitung (100 g Kieselgel, Säule 35×3 $\left.\mathrm{cm}, \mathrm{Hexan} / \mathrm{Et}_{2} \mathrm{O} 10: 1, R_{\mathrm{f}}=0.64\right) 2.36 \mathrm{~g}(99 \%)$ der Titelverbindung 164d als farbloser Feststoff (Smp. $197^{\circ} \mathrm{C}($ Zers. $\left.)\right)$. - IR (KBr): v[ $\left.\mathrm{cm}^{-1}\right]=3020,2925$, 2846, 1512, 1446, 1223, 1167, 976, 842, 820, 560. - ${ }^{1} \mathrm{H}$ NMR: $\delta=0.81$ (m, 1 H, Ch cycloprop.), 0.85 (t, $\left.{ }^{3} J=7.1 \mathrm{~Hz}, 3 \mathrm{H}, \mathrm{CH}_{3}\right), 1.05-1.80$ (m, $\left.2 \mathrm{H}\right), 1.22-$ $1.31(\mathrm{~m}, 6 \mathrm{H}), 1.34-1.46(\mathrm{~m}, 6 \mathrm{H}), 1.67-1.93(\mathrm{~m}, 13 \mathrm{H}), 2.18\left(\mathrm{dd},{ }^{3} J=7.4\right.$, $\left.12.5 \mathrm{~Hz}, 2 \mathrm{H}, \mathrm{CH}_{2}\right), 2.42\left(\mathrm{tt},{ }^{3} \mathrm{~J}=3.0,11.8 \mathrm{~Hz}, 1 \mathrm{H}, \mathrm{CH}\right), 2.70\left(\mathrm{tt},{ }^{3} J=7.4,11.3\right.$ $\mathrm{Hz}, 1 \mathrm{H}, \mathrm{CH}), 3.15$ (d, $\left.{ }^{3} \mathrm{~J}=7.8 \mathrm{~Hz}, 2 \mathrm{H}, \mathrm{CH}_{2} \mathrm{I}\right), 7.12$ (s, $\left.4 \mathrm{H}, \mathrm{Ar}\right) \cdot-{ }^{13} \mathrm{C}$ NMR: $\delta$

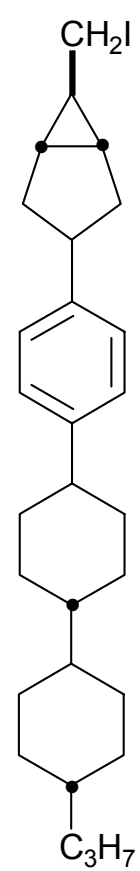
$=14.4\left(\mathrm{CH}_{3}\right), 30.1,30.3,33.6,34.6,36.2\left(2 \mathrm{CH}_{2}\right), 12.5,20.0,39.8\left(\mathrm{CH}_{2}\right), 30.6,126.7,127.1$ (2 CH), 24.4, 37.6, 40.1, 42.9, 43.4, $44.1(\mathrm{CH}), 141.4,145.6(\mathrm{C}) .-\mathrm{C}_{28} \mathrm{H}_{41} \mathrm{I}$ (504.51): ber. $\mathrm{C}$ 66.65, H 8.19, I 25.15\%; gem. C 66.39, H 8.08. 
exo,exo-3-\{[4-(trans-4-n-Pentyl)cyclohexyl]phenyl\}-6-n-

propylbicyclo[3.1.0]hexan (165a): Gemäss AAV 8 wurden $2.28 \mathrm{~g}(5.05 \mathrm{mmol})$ des Jodids 164a und $2.32 \mathrm{ml}$ (3.26 M Lösung, $7.58 \mathrm{mmol})$ EtMgBr unter Katalyse des aus $21 \mathrm{mg}(0.50 \mathrm{mmol}) \mathrm{LiCl}$ und $34 \mathrm{mg}(0.25 \mathrm{mmol}) \mathrm{CuCl}_{2}$ hergestellten Katalysators zur Reaktion gebracht und säulenchromatographisch (300 g Kieselgel, Säule $50 \times 4.5 \mathrm{~cm}$, Hexan, $\left.R_{\mathrm{f}}=0.55\right)$ aufgearbeitet. Es konnten $1.36 \mathrm{~g} \mathrm{(77 \% )}$ ) der Titelverbindung 165a als farbloser Feststoff (Smp. 100-101 ${ }^{\circ} \mathrm{C}$ $(\mathrm{MeOH}))$ isoliert werden. - IR (KBr): $v\left[\mathrm{~cm}^{-1}\right]=2957,2850,1514,1465,1444$, 1378, 1297, 1263, 1212, 1182, 1144, 1112, 1050, 1018, 973, 896, 825, 730, 585. $-{ }^{1} \mathrm{H}$ NMR: $\delta=0.72$ (tt, ${ }^{3} J=3.6,6.8 \mathrm{~Hz}, 1 \mathrm{H}, \mathrm{CH}$ cycloprop.), 0.89 (t, ${ }^{3} J=7.0$ $\left.\mathrm{Hz}, 3 \mathrm{H}, \mathrm{CH}_{3}\right), 0.92$ (t, $\left.{ }^{3} J=7.5 \mathrm{~Hz}, 3 \mathrm{H}, \mathrm{CH}_{3}\right), 1.05-1.50(\mathrm{~m}, 16 \mathrm{H}), 1.71-1.81$

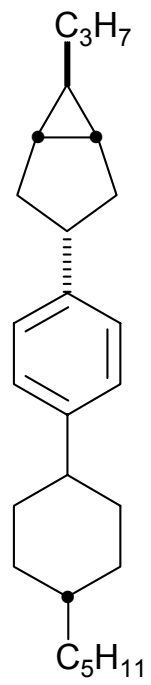
(m, $1 \mathrm{H}, \mathrm{CH}), 1.84\left(\mathrm{dd},{ }^{3} J=8.0,8.8 \mathrm{~Hz}, 8 \mathrm{H}, 4 \mathrm{CH}_{2}\right), 2.11\left(\mathrm{dd},{ }^{3} J=7.3,12.3 \mathrm{~Hz}, 2 \mathrm{H}, \mathrm{CH}_{2}\right)$, $2.42\left(\mathrm{tt},{ }^{3} J=3.1,12.0 \mathrm{~Hz}, 1 \mathrm{H}, \mathrm{CH}\right), 2.67\left(\mathrm{tt},{ }^{3} J=7.5,11.0 \mathrm{~Hz}, 1 \mathrm{H}, \mathrm{CH}\right), 7.09\left(\mathrm{~d},{ }^{3} J=7.6 \mathrm{~Hz}\right.$, $2 \mathrm{H}, \mathrm{Ar}), 7.13\left(\mathrm{~d},{ }^{3} J=7.6 \mathrm{~Hz}, 2 \mathrm{H}, \mathrm{Ar}\right) .-{ }^{13} \mathrm{C} \mathrm{NMR}: \delta=14.07,14.12\left(\mathrm{CH}_{3}\right), 33.6,34.4,36.4$ (2 $\left.\mathrm{CH}_{2}\right), 22.69,22.71,26.6,32.2,35.1,37.7\left(\mathrm{CH}_{2}\right), 24.1,126.6,127.1(2 \mathrm{CH}), 19.7,37.3$, 40.6, $44.1(\mathrm{CH}), 142.4,145.4(\mathrm{C}) .-\mathrm{C}_{26} \mathrm{H}_{40}$ (352.58): ber. C 88.56, $\mathrm{H} 11.44 \%$; gem C 88.85, H 11.25\%.

exo,exo-6-n-Heptyl-3-(3,4,5-trifluorophenyl)bicyclo[3.1.0] hexan (165b):

Gemäss AAV 8 wurden $2.55 \mathrm{~g}$ (7.24 mmol) des Jodids 164b und $16.2 \mathrm{ml}$ (0.67 M Lösung, $10.9 \mathrm{mmol}) n-\mathrm{C}_{6} \mathrm{H}_{13} \mathrm{MgBr}$ unter Katalyse des aus $30 \mathrm{mg}$ $\mathrm{LiCl}$ und $49 \mathrm{mg} \mathrm{CuCl} 2$ bereiteten Katalysators umgesetzt und nach säulenchromatographischer Aufarbeitung (100 g Kieselgel, Säule $35 \times 3 \mathrm{~cm}$, Hexan, $\left.R_{\mathrm{f}}=0.60\right) 1.91 \mathrm{~g}(85 \%)$ der Titelverbindung $\mathbf{1 6 5 b}$ als farbloses Öl (Smp. ca. $10-12{ }^{\circ} \mathrm{C}$ ) isoliert. $-{ }^{1} \mathrm{H}$ NMR: $\delta=0.67\left(\mathrm{tt},{ }^{3} \mathrm{~J}=3.3\right.$, $6.7 \mathrm{~Hz}, 1 \mathrm{H}, \mathrm{CH}$ cycloprop.), 0.89 (t, $\left.{ }^{3} J=6.5 \mathrm{~Hz}, 3 \mathrm{H}, \mathrm{CH}_{3}\right), 1.10\left(\mathrm{t},{ }^{3} J=\right.$

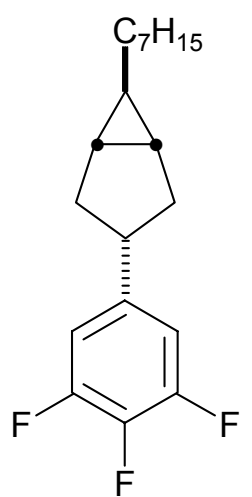
$6.3 \mathrm{~Hz}, 2 \mathrm{H}), 1.17\left(\mathrm{t},{ }^{3} J=6.8 \mathrm{~Hz}, 2 \mathrm{H}\right), 1.21-1.41(\mathrm{~m}, 10 \mathrm{H}), 1.73\left(\mathrm{ddd},{ }^{3} J=3.3,11.0,12.1\right.$ $\left.\mathrm{Hz}, 2 \mathrm{H}, \mathrm{CH}_{2}\right), 2.13\left(\mathrm{dd},{ }^{3} J=7.5,12.1 \mathrm{~Hz}, 2 \mathrm{H}, \mathrm{CH}_{2}\right), 2.64\left(\mathrm{tt},{ }^{3} J=7.5,11.0 \mathrm{~Hz}, 1 \mathrm{H}, \mathrm{CH}\right)$, $6.80\left(\mathrm{ddd},{ }^{3} J=3.3,7.3,13.7 \mathrm{~Hz}, 2 \mathrm{H}, \mathrm{Ar}\right) .-{ }^{13} \mathrm{C} \mathrm{NMR}: \delta=14.1\left(\mathrm{CH}_{3}\right), 36.3\left(2 \mathrm{CH}_{2}\right), 22.7$, 29.4, 29.5, 29.6, 31.9, $32.9\left(\mathrm{CH}_{2}\right), 24.2,110.7$ (ddd, $\left.J=7.0,13.9,21.2 \mathrm{~Hz}\right), 126.6,128.0(2$ $\mathrm{CH}), 20.0,40.7(\mathrm{CH}), 151.4(\mathrm{ddd}, J=4.4,10.1,249.3 \mathrm{~Hz})(2 \mathrm{C}), 135.7,137.2(\mathrm{dt}, J=4.7$, $12.5 \mathrm{~Hz}) 139.1$ (dt, $J=14.9,241.5 \mathrm{~Hz}), 145.6(\mathrm{C})$. 
exo,exo-6-n-Heptyl-3-[4-(3,4,5-

trifluorophenyl)phenyl]bicyclo[3.1.0]hexan (165c): Gemäss $\quad$ AAV 8 wurden $2.34 \mathrm{~g}(5.47 \mathrm{mmol})$ des Jodids $164 \mathrm{c}$ mit $16.3 \mathrm{ml}$ (0.67 M Lösung, $10.9 \mathrm{mmol}) n-\mathrm{C}_{6} \mathrm{H}_{13} \mathrm{MgBr}-\mathrm{Lösung}$ unter Katalyse des aus $30 \mathrm{mg} \mathrm{LiCl}$ und $49 \mathrm{mg} \mathrm{CuCl} 2$ frisch bereiteten Katalysators zur Reaktion gebracht und nach säulenchromatographischer Aufarbeitung (120 g Kieselgel, Säule $40 \times 3 \mathrm{~cm}$, Hexan, $\left.R_{\mathrm{f}}=0.51\right)$ und $1.67 \mathrm{~g}(85 \%)$ der Titelverbindung $\mathbf{1 6 5 c}$ $(1.67 \mathrm{~g}, 85 \%)$ als farbloses bei $0{ }^{\circ} \mathrm{C}$ erstarrendes Ö1 (Smp. $\left.34{ }^{\circ} \mathrm{C}(\mathrm{MeOH})\right)$ isoliert. - IR (KBr): $v\left[\mathrm{~cm}^{-1}\right]=2923,1613,1536,1509,1364,1250(\mathrm{CF})$, 1029, 826, 764, 561, 534. $-{ }^{1} \mathrm{H}$ NMR: $\delta=0.77\left(\mathrm{tt},{ }^{3} J=3.5,6.8 \mathrm{~Hz}, 1 \mathrm{H}, \mathrm{CH}\right.$

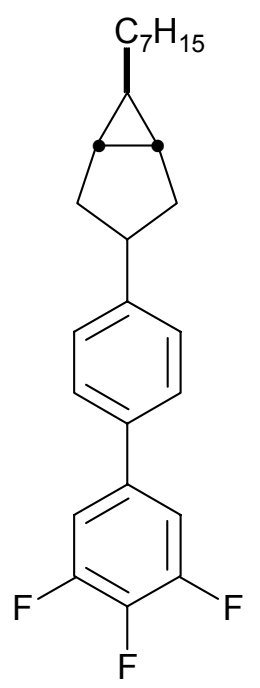
cycloprop.), 0.93 (t, $\left.{ }^{3} J=6.8 \mathrm{~Hz}, 3 \mathrm{H}, \mathrm{CH}_{3}\right), 1.15(\mathrm{~m}, 2 \mathrm{H}), 1.22\left(\mathrm{t},{ }^{3} \mathrm{~J}=6.8 \mathrm{~Hz}, 2 \mathrm{H}\right), 1.25-$ 1.45 (m, $10 \mathrm{H}), 1.87\left(\mathrm{ddd},{ }^{3} J=3.3,11.0,12.3 \mathrm{~Hz}, 2 \mathrm{H}, \mathrm{CH}_{2}\right), 2.20\left(\mathrm{dd},{ }^{3} J=7.5,12.3 \mathrm{~Hz}, 2 \mathrm{H}\right.$, $\left.\mathrm{CH}_{2}\right), 2.78\left(\mathrm{tt},{ }^{3} \mathrm{~J}=7.5,11.0 \mathrm{~Hz}, 1 \mathrm{H}, \mathrm{CH}\right), 7.17\left(\mathrm{dd},{ }^{3} \mathrm{~J}=6.5,8.8 \mathrm{~Hz}, 2 \mathrm{H}, \mathrm{Ar}\right), 7.29\left(\mathrm{~d},{ }^{3} J=\right.$ $8.3 \mathrm{~Hz}, 2 \mathrm{H}, \mathrm{Ar}), 7.41\left(\mathrm{~d},{ }^{3} J=8.3 \mathrm{~Hz}, 2 \mathrm{H}, \mathrm{Ar}\right) .-{ }^{13} \mathrm{C} \mathrm{NMR}: \delta=14.1\left(\mathrm{CH}_{3}\right), 22.7,24.2,29.4$, 29.5, 29.6, 31.9, $32.9\left(\mathrm{CH}_{2}\right), 36.3\left(2 \mathrm{CH}_{2}\right), 110.7$ (ddd, $\left.J=7.0,13.9,21.2 \mathrm{~Hz}\right), 126.6,128.0(2$ CH), 20.0, 40.7 (CH), 151.4 (ddd, $J=4.4,10.1,249.3 \mathrm{~Hz})(2 \mathrm{C}), 135.7,137.2$ (dt, $J=4.7$, $12.5 \mathrm{~Hz}) 139.1$ (dt, $J=14.9,241.5 \mathrm{~Hz}$ ), $145.6(\mathrm{C}) .-\mathrm{C}_{25} \mathrm{H}_{19} \mathrm{~F}_{3}$ (386.48): ber. $\mathrm{C} 77.69, \mathrm{H}$ 7.56\%; gem. C 77.37, H 7.60\%.

exo,exo-6-n-Heptyl-3-[\{trans-4-[(trans-4-npropyl)cyclohexyl]cyclohexyl'phenyl]bicyclo-[3.1.0] hexan (165d): Gemäß AAV 8 wurden $2.31 \mathrm{~g}(4.58 \mathrm{mmol})$ des Jodids 164d mit $13.7 \mathrm{ml}$ (0.67 M Lösung , 9.16) $n-\mathrm{C}_{6} \mathrm{H}_{13} \mathrm{MgBr}$ unter Katalyse des aus $30 \mathrm{mg} \mathrm{LiCl}$ und $49 \mathrm{mg} \mathrm{CuCl}$ frisch zubereiteten Katalysators zur Reaktion gebracht und nach säulenchromatographischer Aufarbeitung (300 g Kieselgel, Säule $50 \times 4.5 \mathrm{~cm}$, Hexan, $R_{\mathrm{f}}=0.57$.) $1.65 \mathrm{~g}(78 \%)$ der Titelverbindung $165 \mathrm{~d}$ als farbloser Feststoff (Smp. $\left.237-239{ }^{\circ} \mathrm{C}\left(\mathrm{MeOH} / \mathrm{CHCl}_{3} 10: 1\right)\right)$ isoliert. $-\mathrm{IR}(\mathrm{KBr}): v\left[\mathrm{~cm}^{-1}\right]=3019$, 2957, 2850, 1513, 1466, 1441, 1050, 997, 892, 818, 722, 549. $-{ }^{1} \mathrm{H}$ NMR: $\delta=$ 0.71 (tt, ${ }^{3} J=2.9,6.5 \mathrm{~Hz}, 1 \mathrm{H}, \mathrm{CH}$ cycloprop.), 0.87 ( $\mathrm{t},{ }^{3} J=7.3 \mathrm{~Hz}, 3 \mathrm{H}, \mathrm{CH}_{3}$ ), $0.89\left(\mathrm{t},{ }^{3} \mathrm{~J}=6.7 \mathrm{~Hz}, 3 \mathrm{H}, \mathrm{CH}_{3}\right), 0.98-1.12(\mathrm{~m}, 12 \mathrm{H}), 1.12-1.52(\mathrm{~m}, 13 \mathrm{H}), 1.61$ (br. S, $2 \mathrm{H}), 1.67-2.00(\mathrm{~m}, 12 \mathrm{H}), 2.11\left(\mathrm{dd},{ }^{3} J=7.3,12.5 \mathrm{~Hz}, 2 \mathrm{H}, \mathrm{CH}_{2}\right), 2.40$ (tt, ${ }^{3} J=3.3,11.8 \mathrm{~Hz}, 1 \mathrm{H}, \mathrm{CH}$ ), $2.68\left(\mathrm{tt},{ }^{3} J=7.3,11.0 \mathrm{~Hz}, 1 \mathrm{H}, \mathrm{CH}\right.$ ), 7.11 (br.s, (1) $4 \mathrm{H}, \mathrm{Ar}) \cdot-{ }^{13} \mathrm{C}$ NMR: $\delta=14.1,14.4\left(\mathrm{CH}_{3}\right), 30.1,30.4,33.6,34.6,36.4\left(2 \mathrm{CH}_{2}\right), 20.0,22.7$, 
29.4, 29.5, 29.6, 31.9, 32.9, $39.8\left(\mathrm{CH}_{2}\right), 24.2,126.6,127.1(2 \mathrm{CH}), 19.9,37.6,40.6,42.9$, 43.4, $44.2(\mathrm{CH}), 142.4,145.4(\mathrm{C})$. 


\section{F. Spektrenanhang}

\section{1. ${ }^{1}$ H-NMR-Spektren}

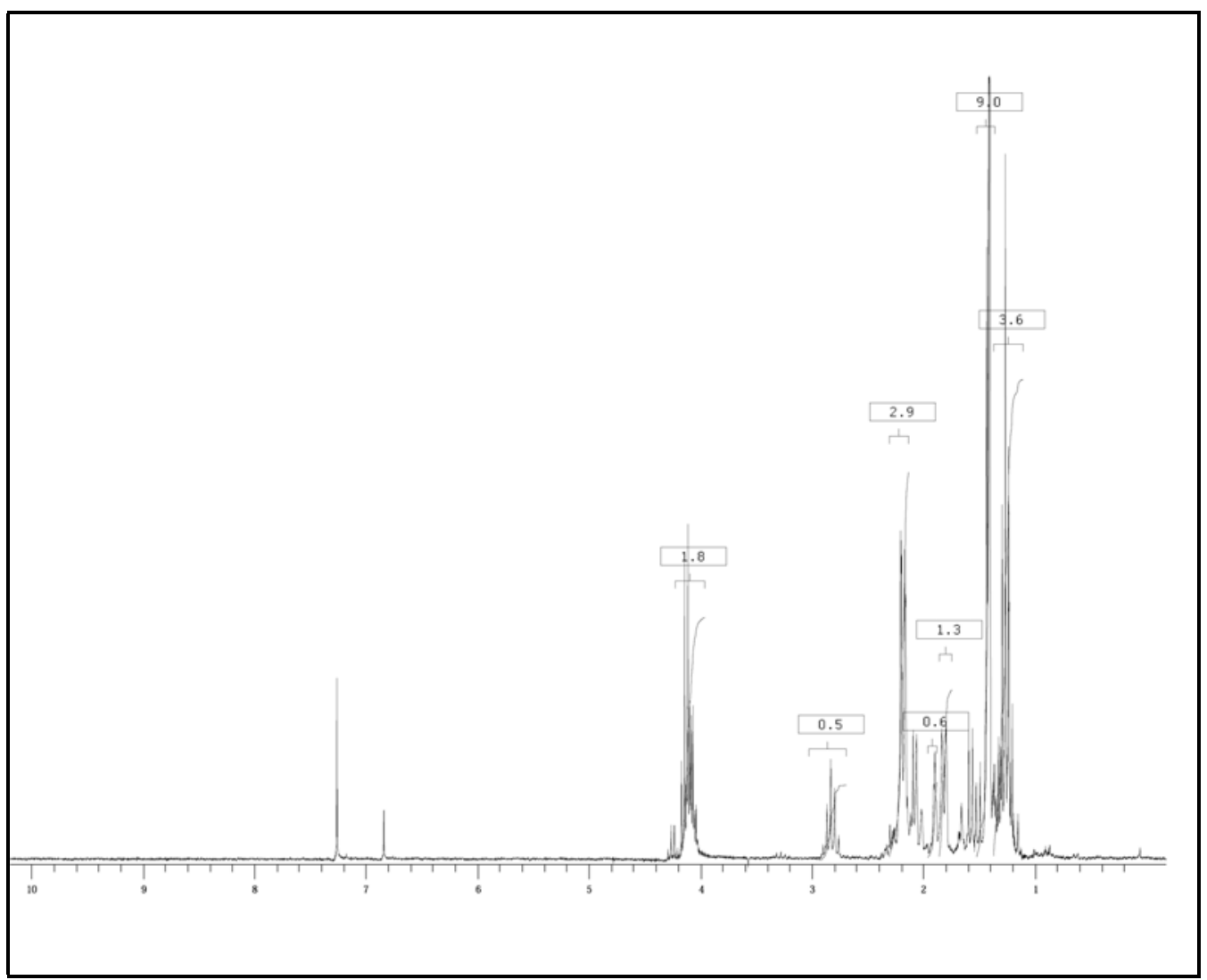

exo,exo-Bicyclo[3.1.0]hexan-3,6-dicarbonsäure-3-tbutylester-6-ethylester 33a

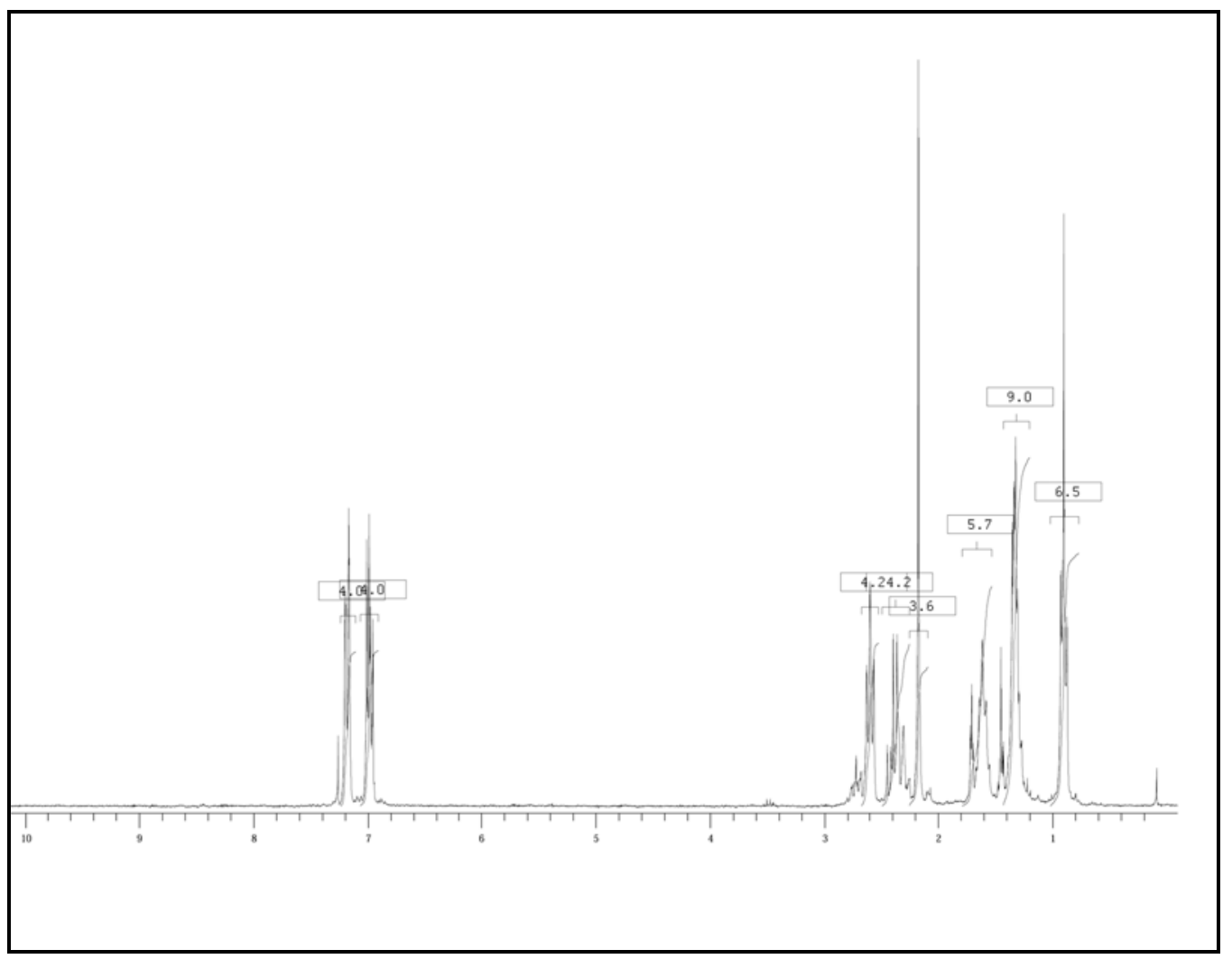

exo,exo-Bicyclo[3.1.0]hexan-3,6-dicarbonsäure-3,6-di(4-npentylphenyl)ester 35a 


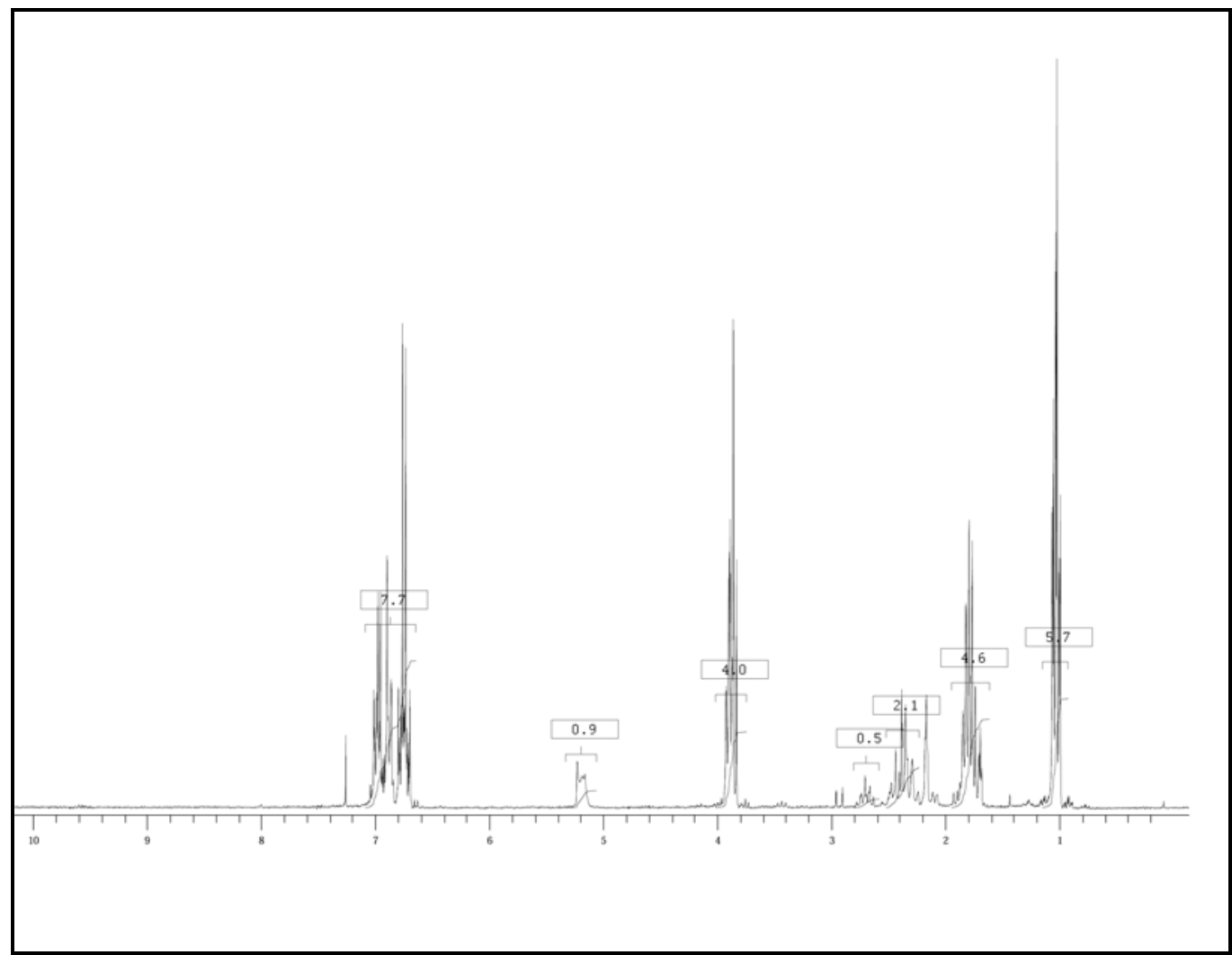

exo,exo-Bicyclo[3.1.0]hexan-3,6-dicarbonsäure-3,6-di(4-npropyloxyphenyl)ester 35b

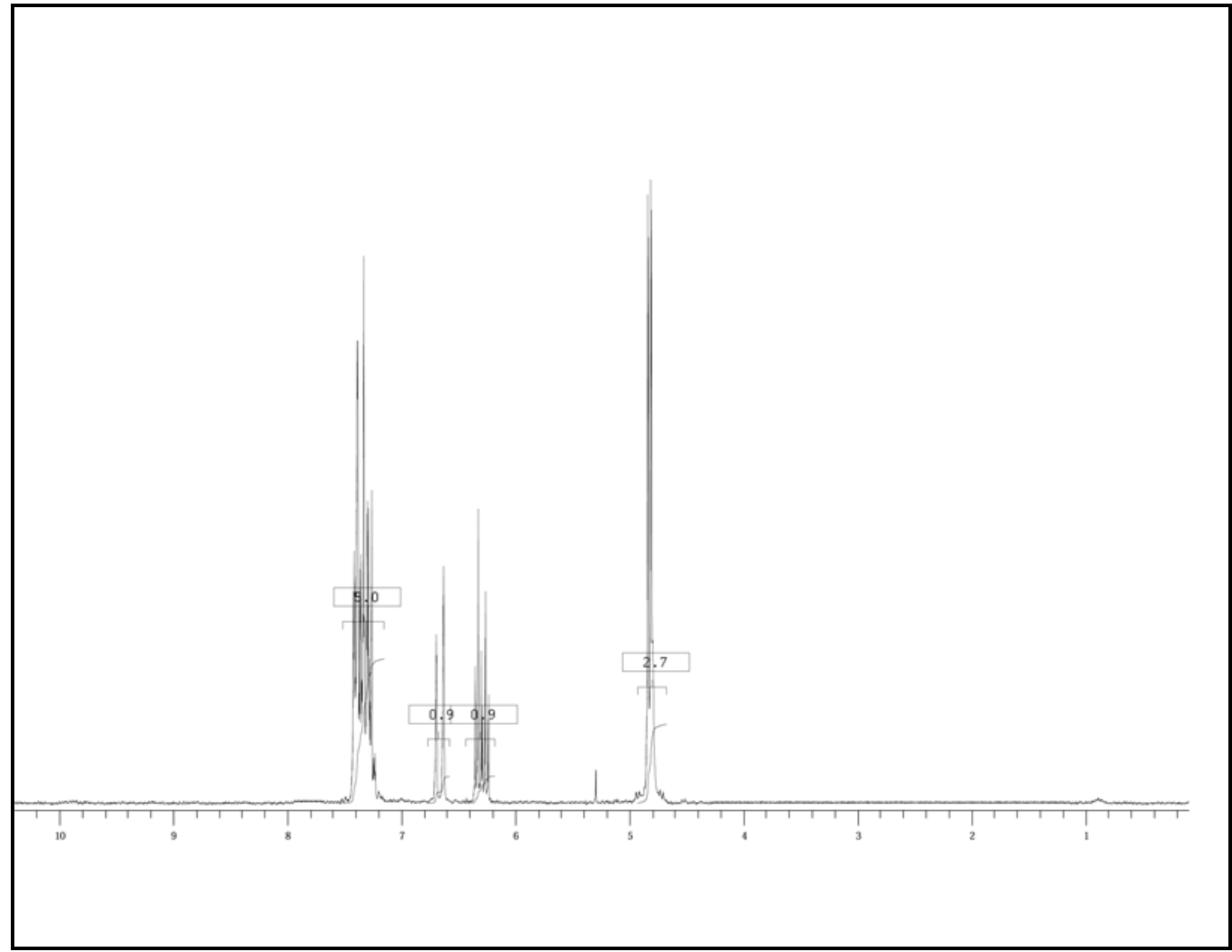

Cinnamyldiazoacetat 53 


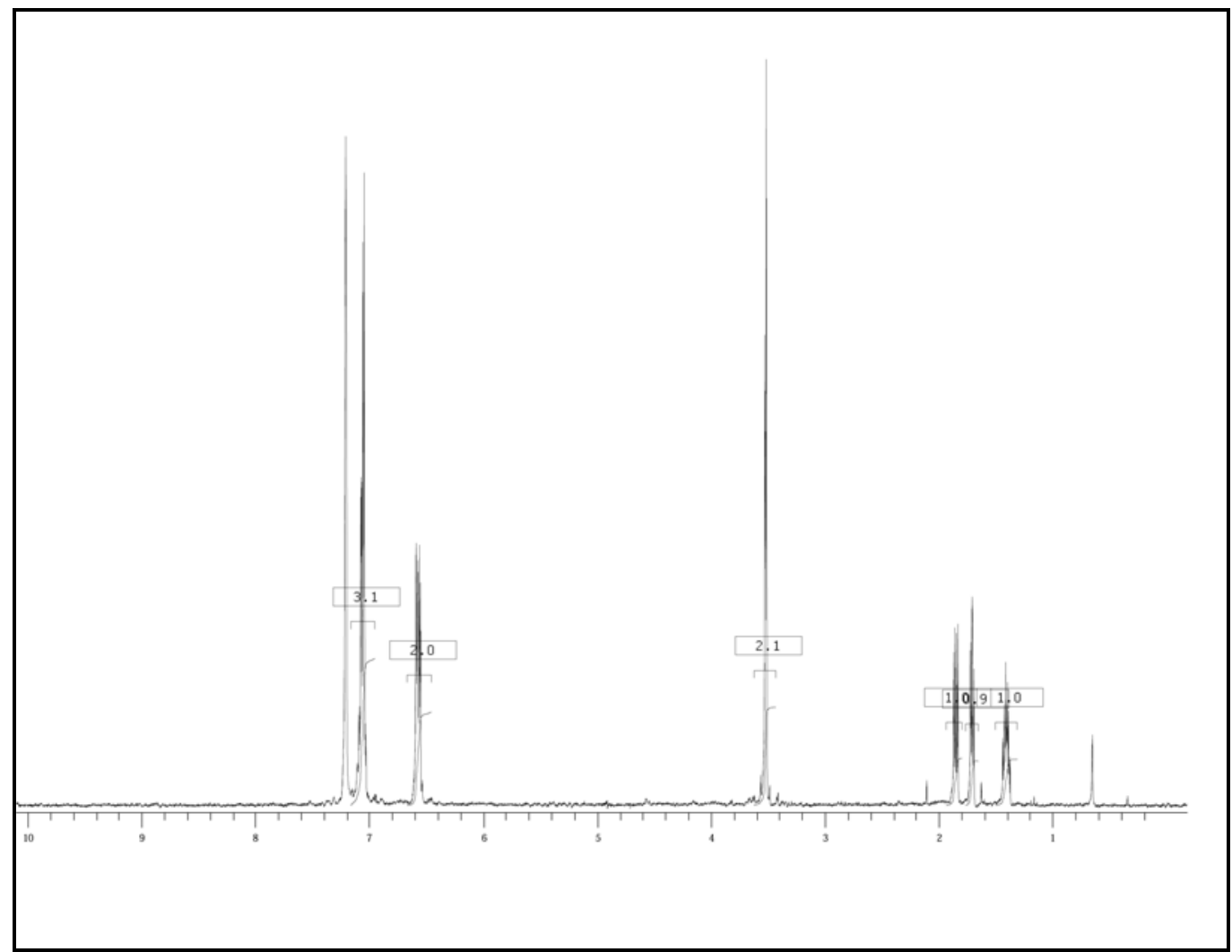

2-Oxo-6-phenyl-3-oxabicyclo[3.1.0]hexan 54

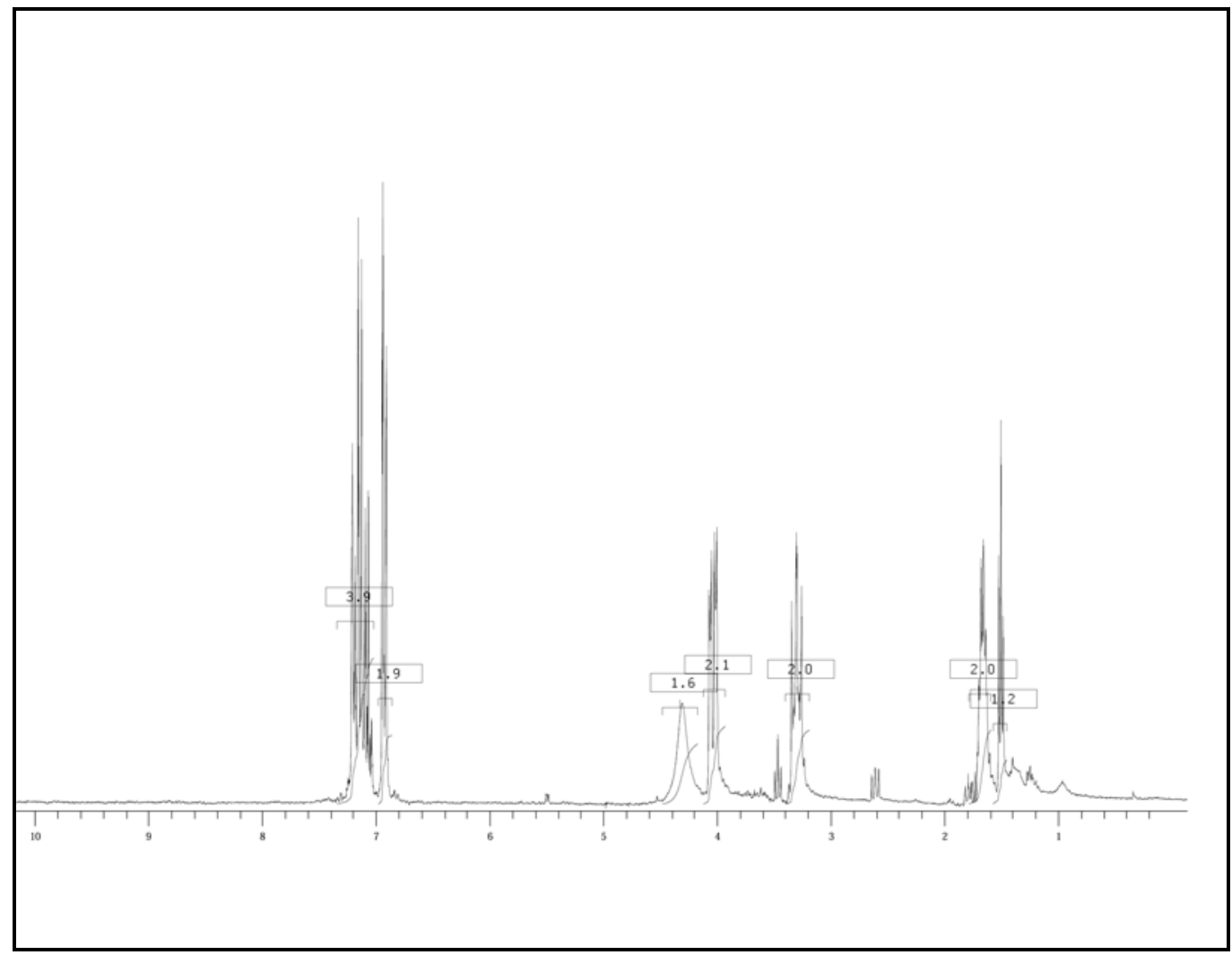

2-Hydroxymethyl-3-phenylcyclopropylmethanol 60 


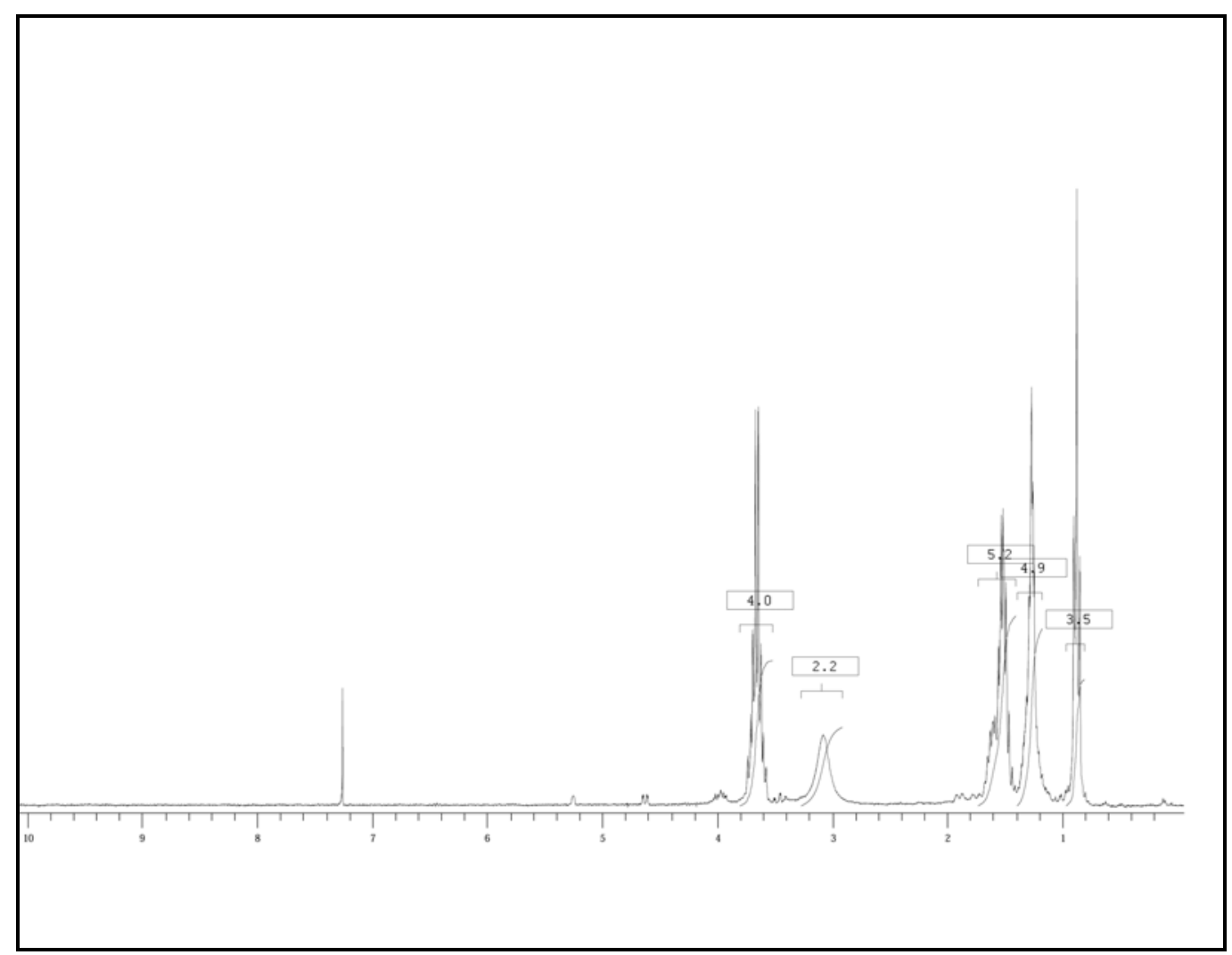

3-nPropyl-1,5-pentadiol 71

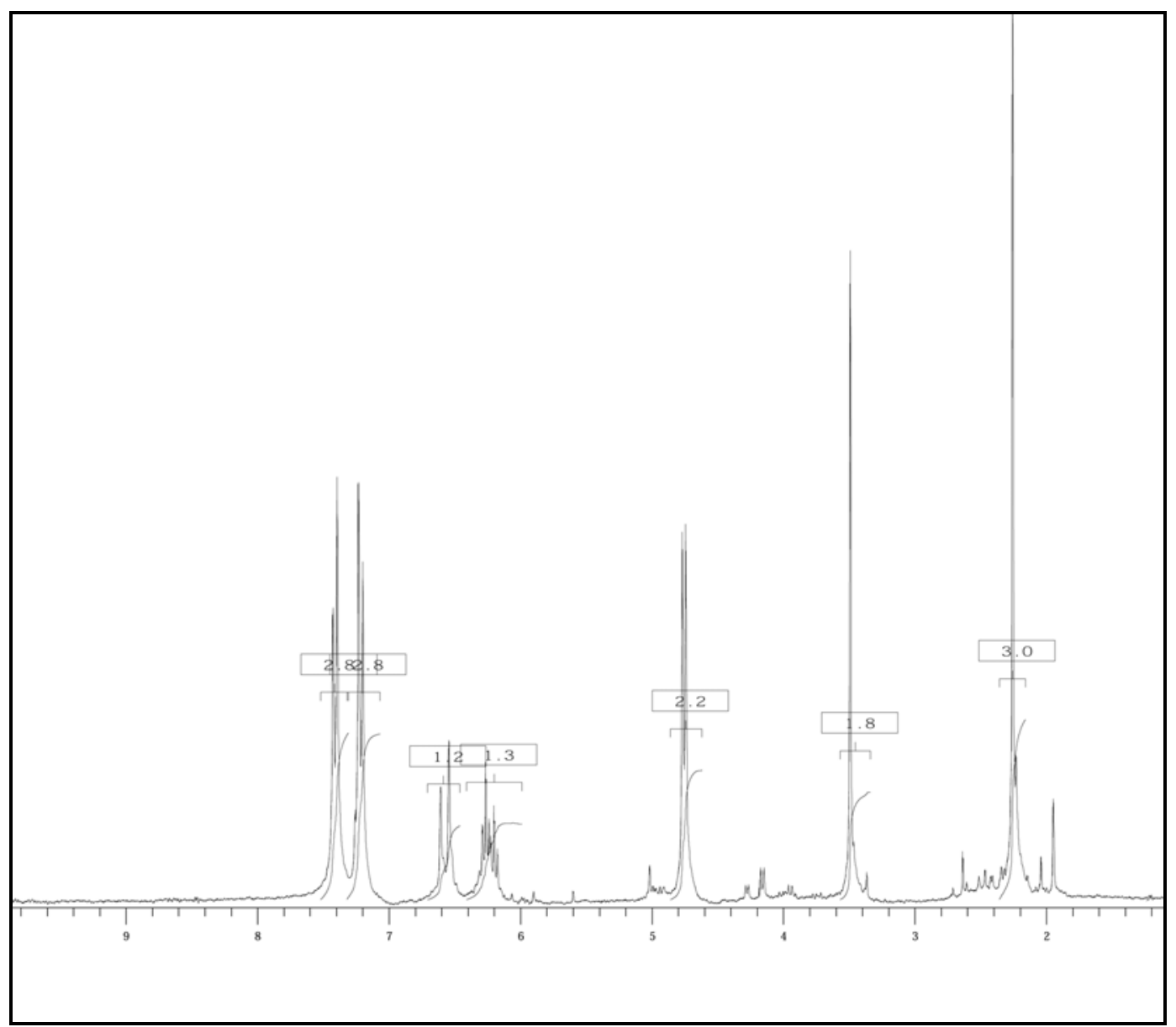

3-nPropyl-1,5-pentadiol 71a 


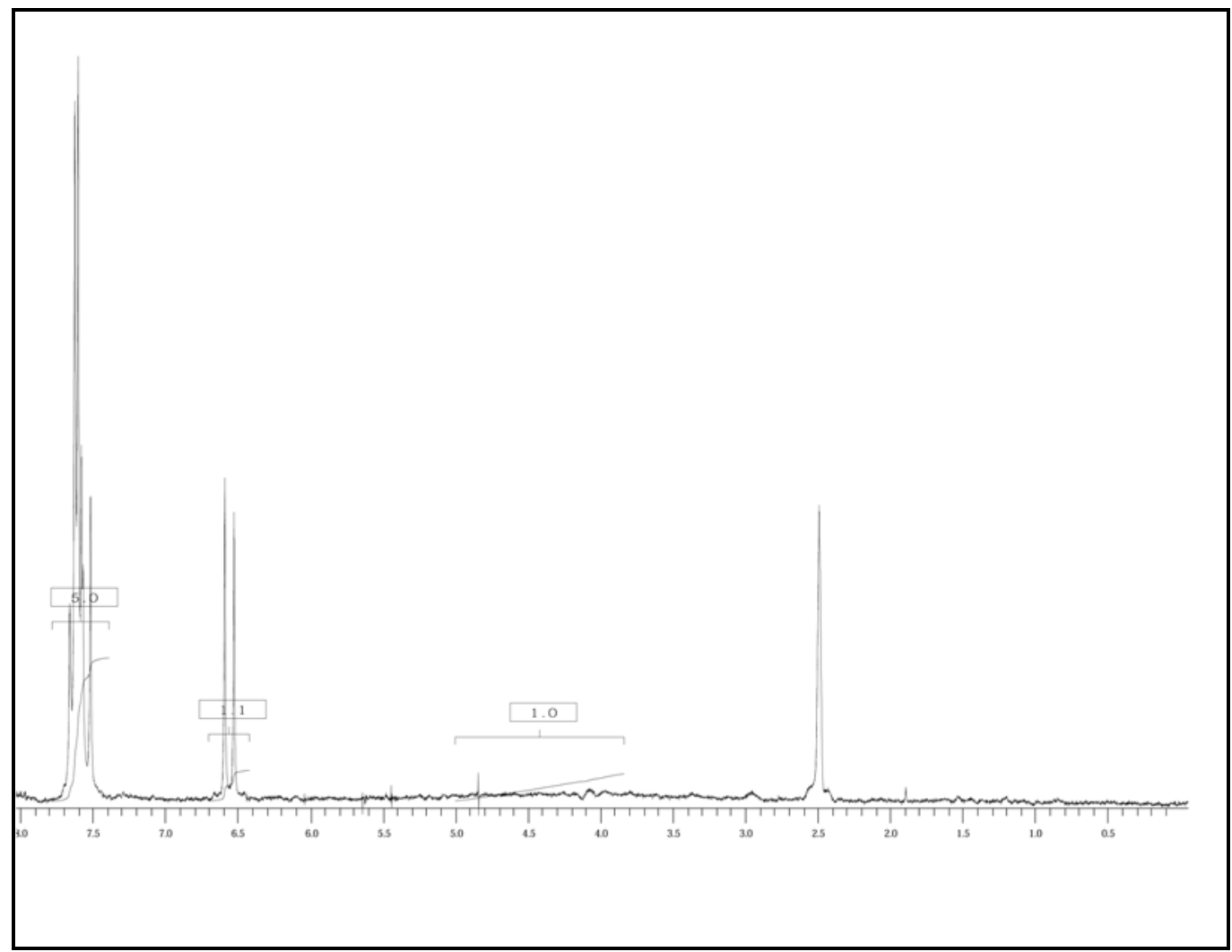

4-Bromzimtsäure 73

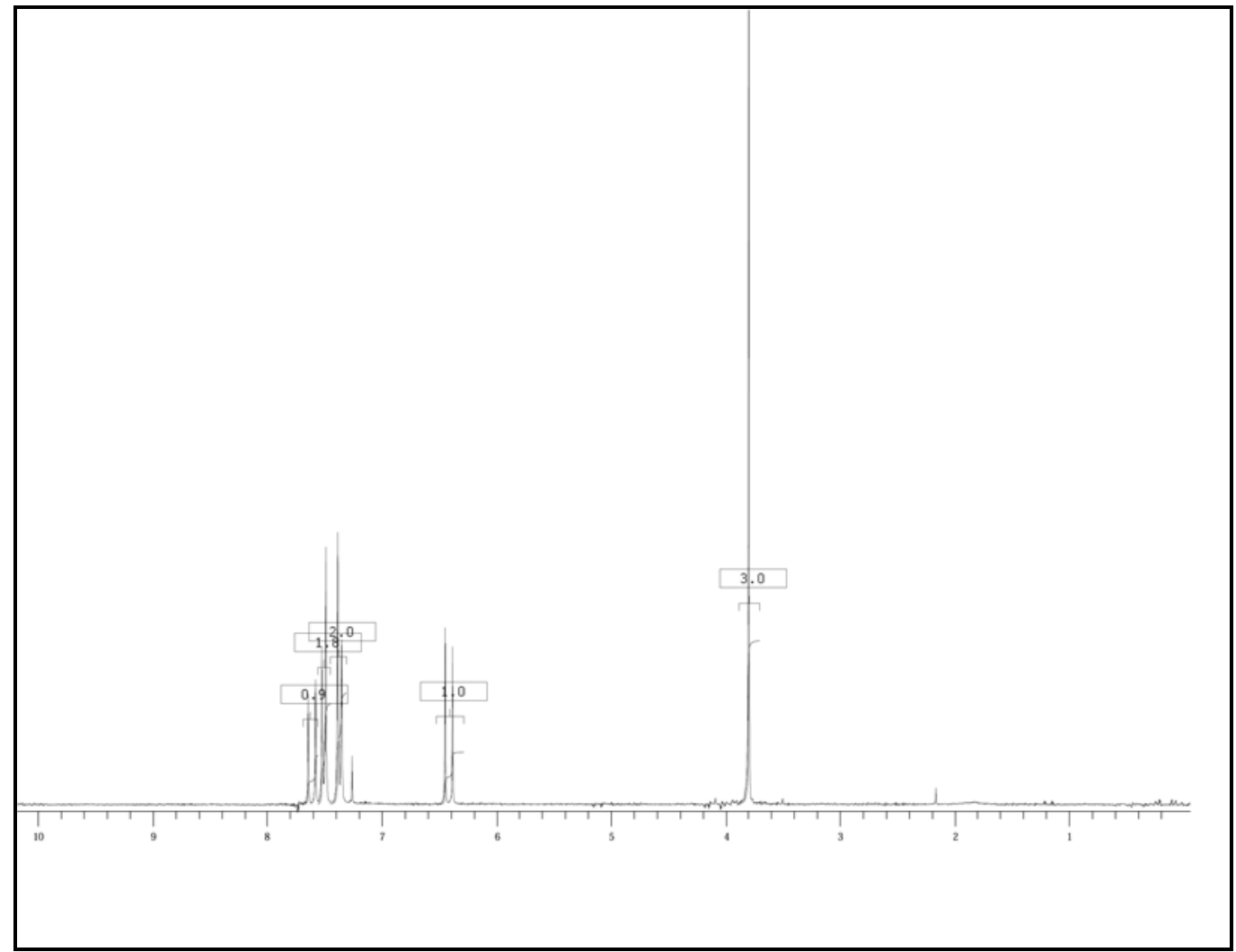

4-Bromzimtsäuremethylester $\mathbf{7 4}$ 
110

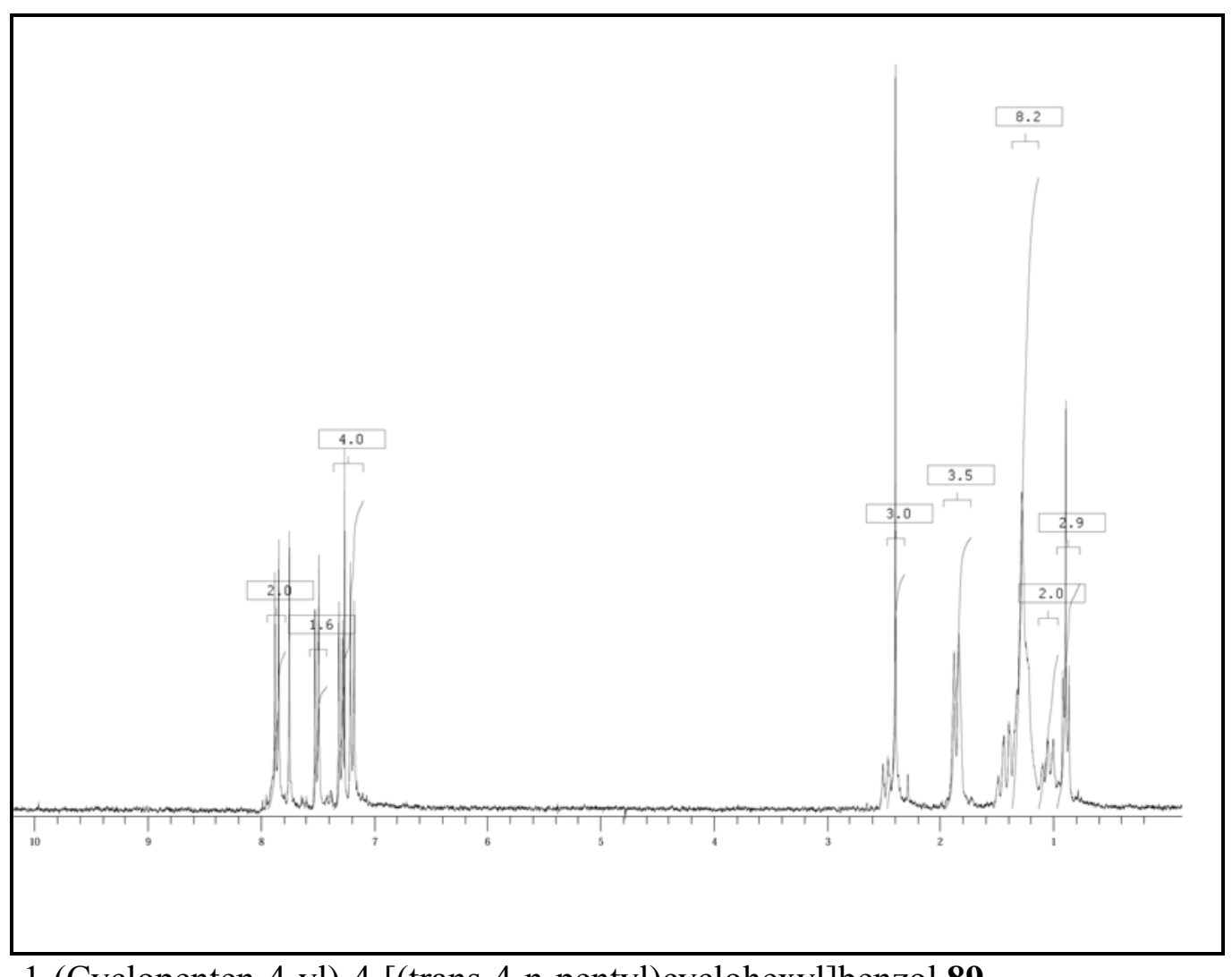

1-(Cyclopenten-4-yl)-4-[(trans-4-n-pentyl)cyclohexyl]benzol 89

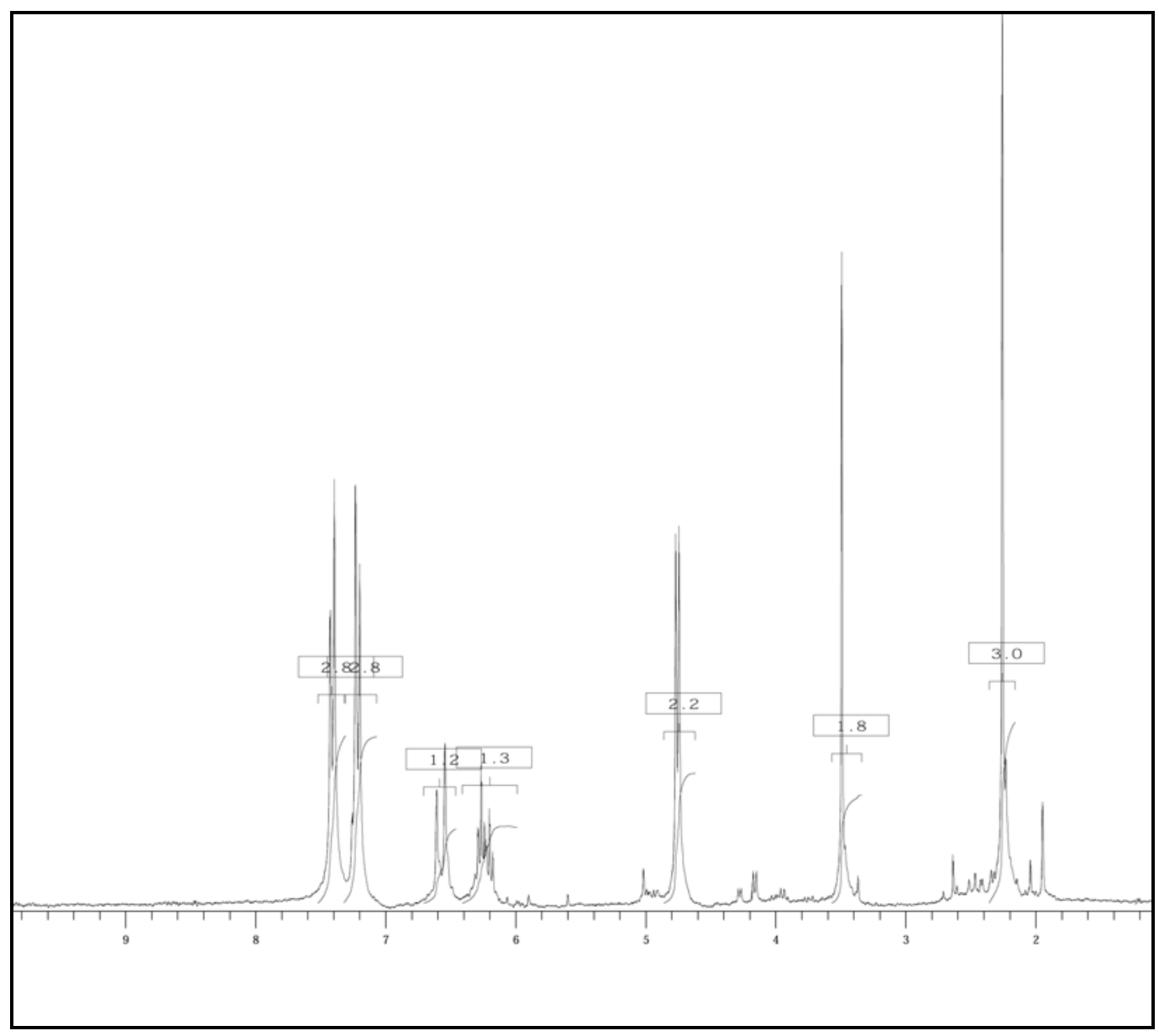

4-Bromcinnamylacetoacetat $\mathbf{7 6}$ 


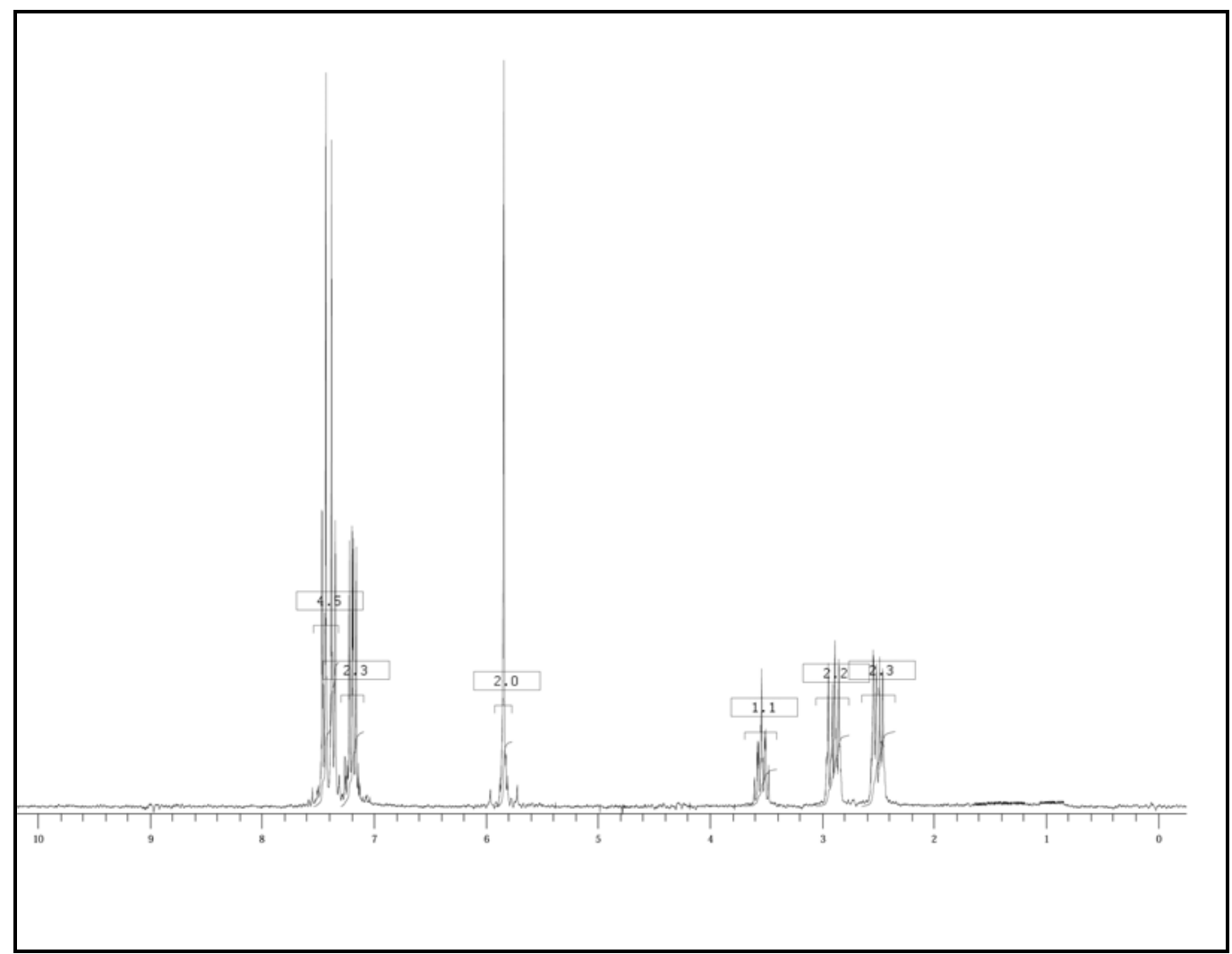

4-[4-(3,4,5-Trifluorophenyl)phenyl]cyclopenten 90

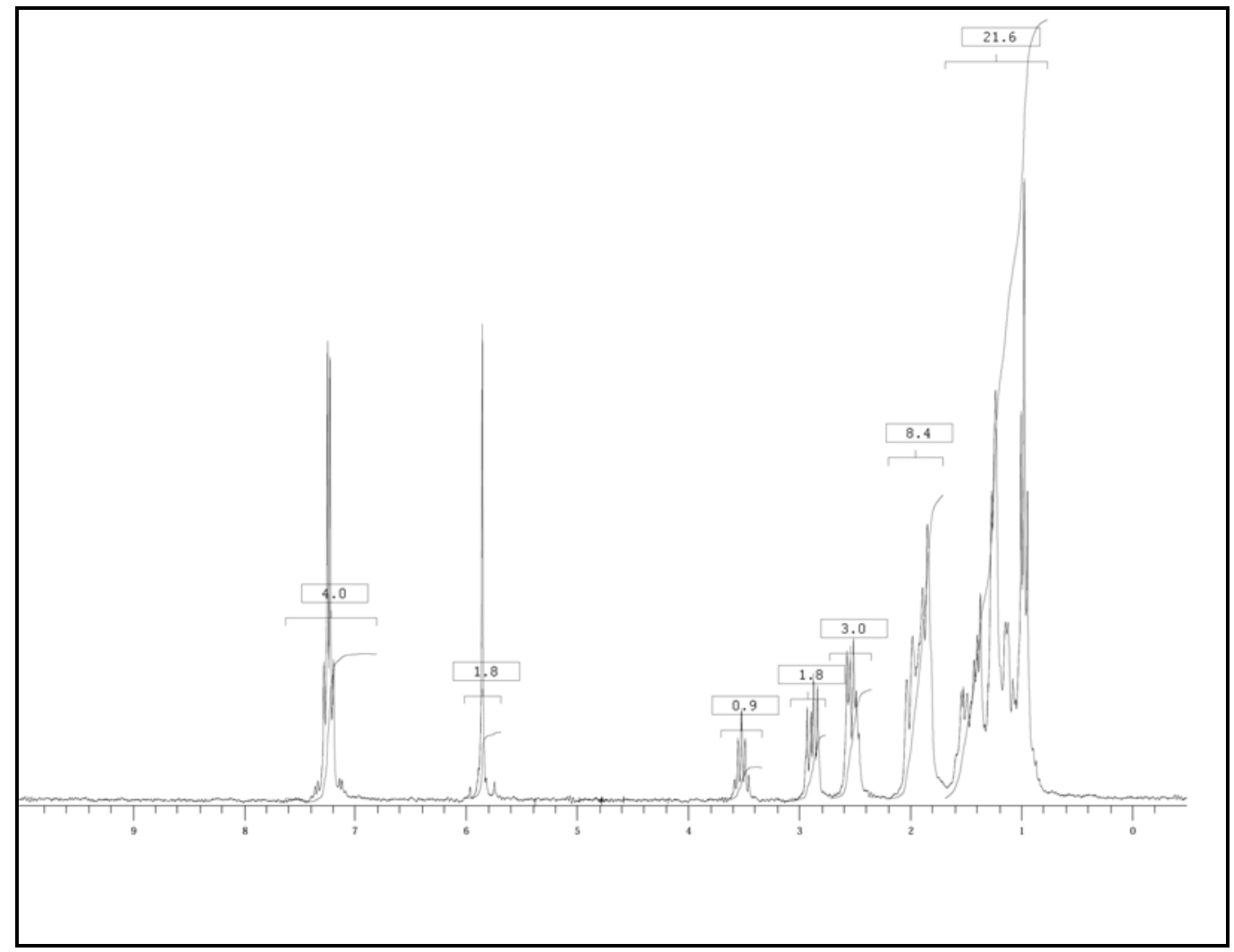

1-(Cyclopenten-4-yl)-4-\{trans-4-[(trans-4-n-propyl)cyclohexyl]cyclohexyl\} benzol 91 


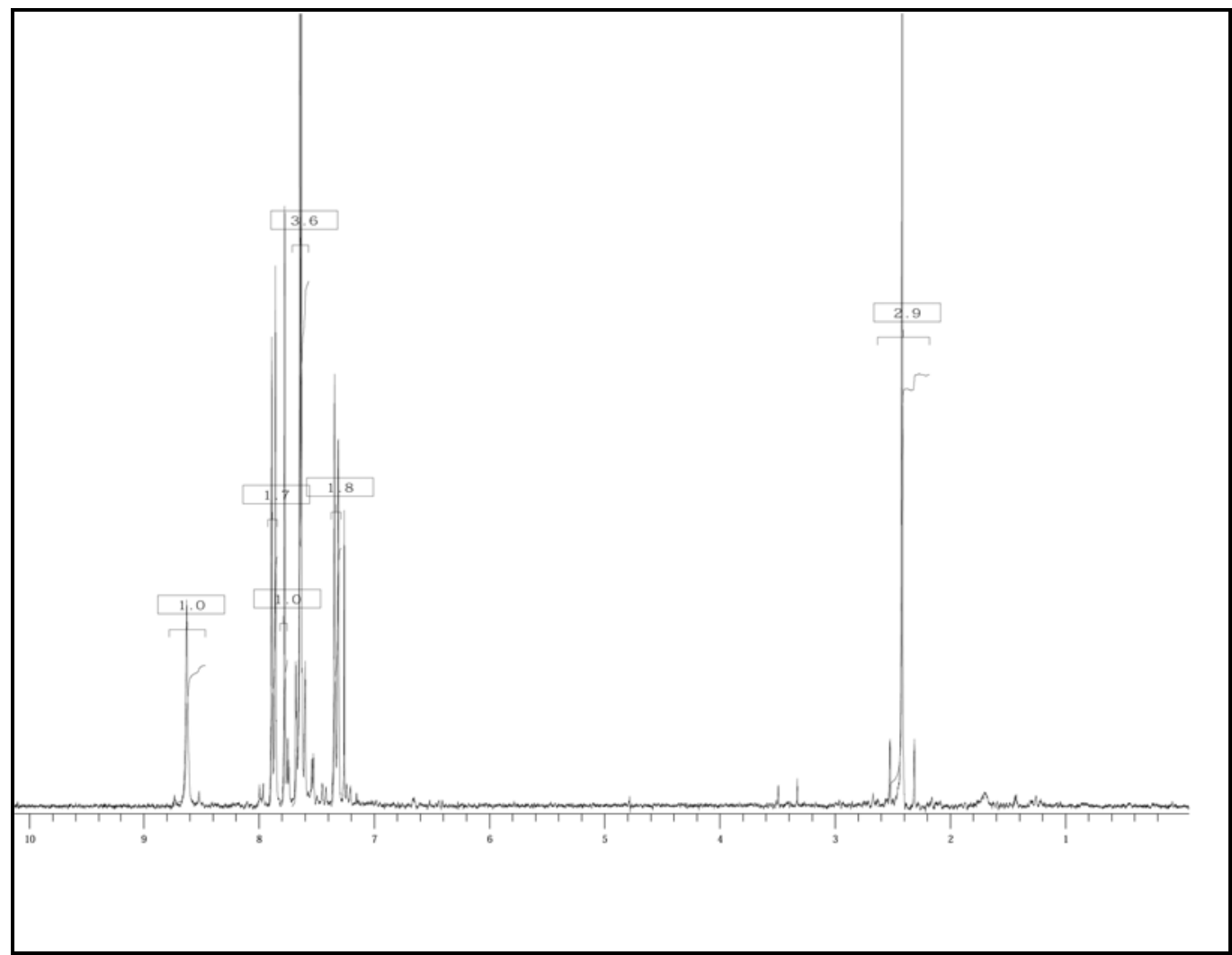

4-Cyanobenzaldehydtosylhydrazon 142a

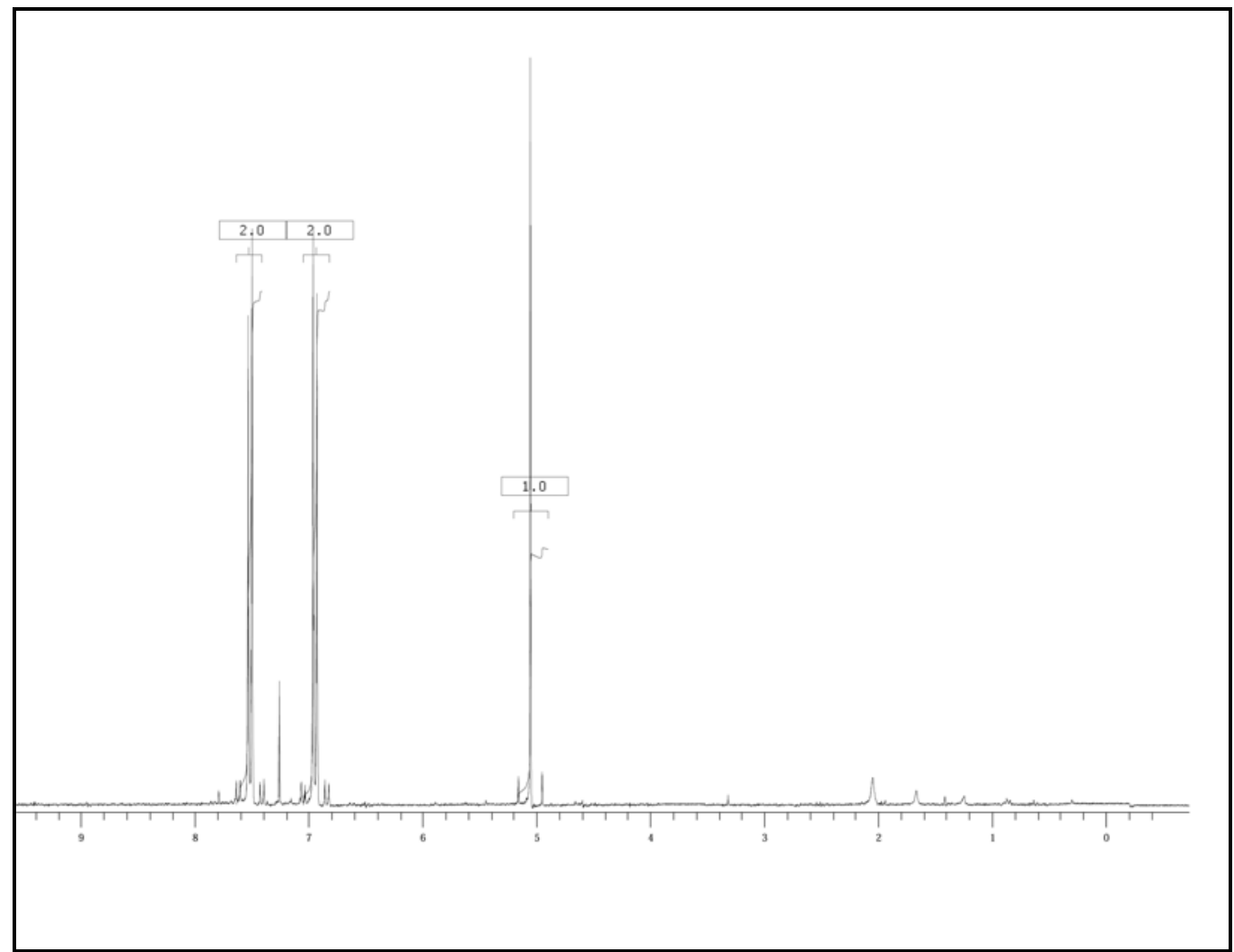

4-Diazomethylbenzonitril 143a 


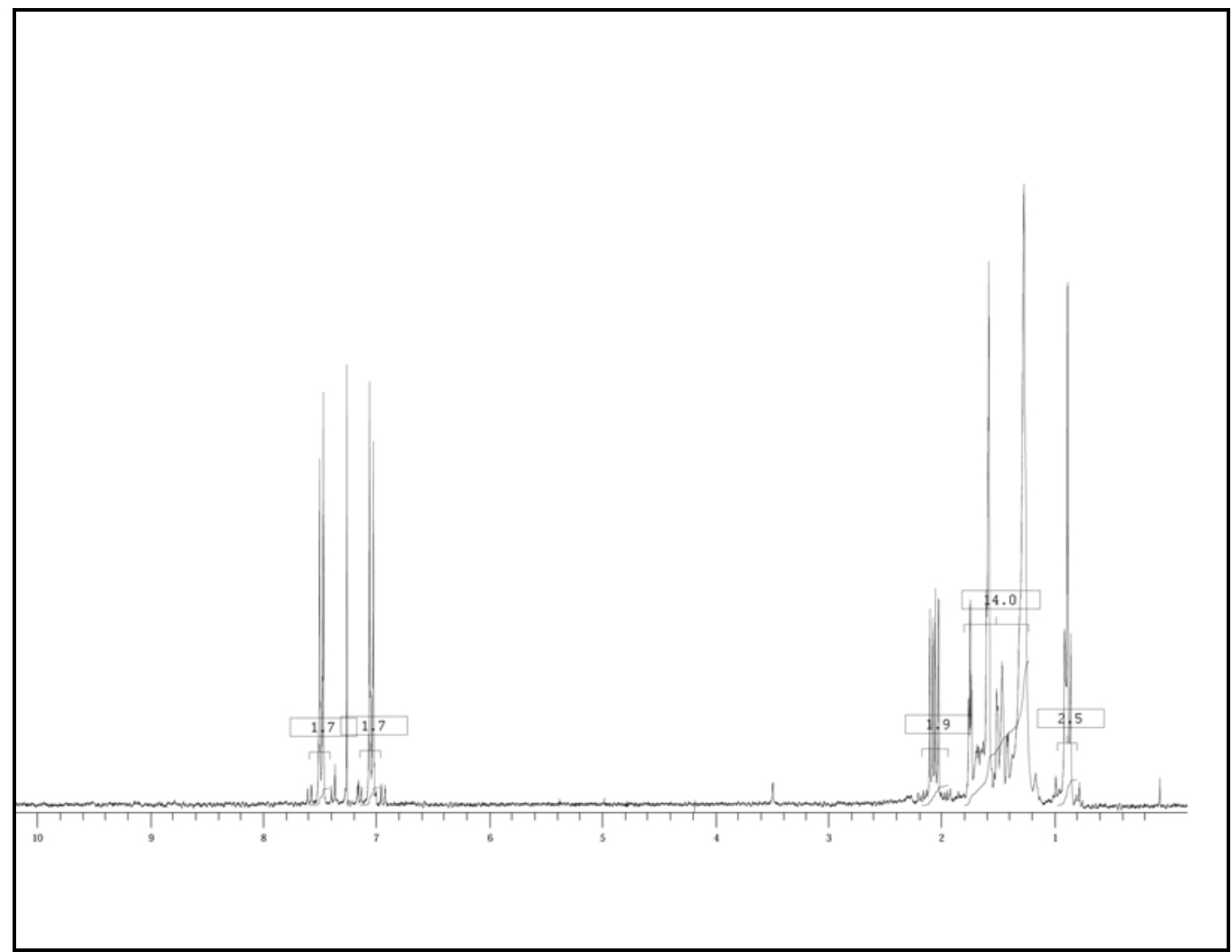

exo,exo-6-(4-Cyanophenyl)-3-npentylbicyclo[3.1.0]hexan 155a

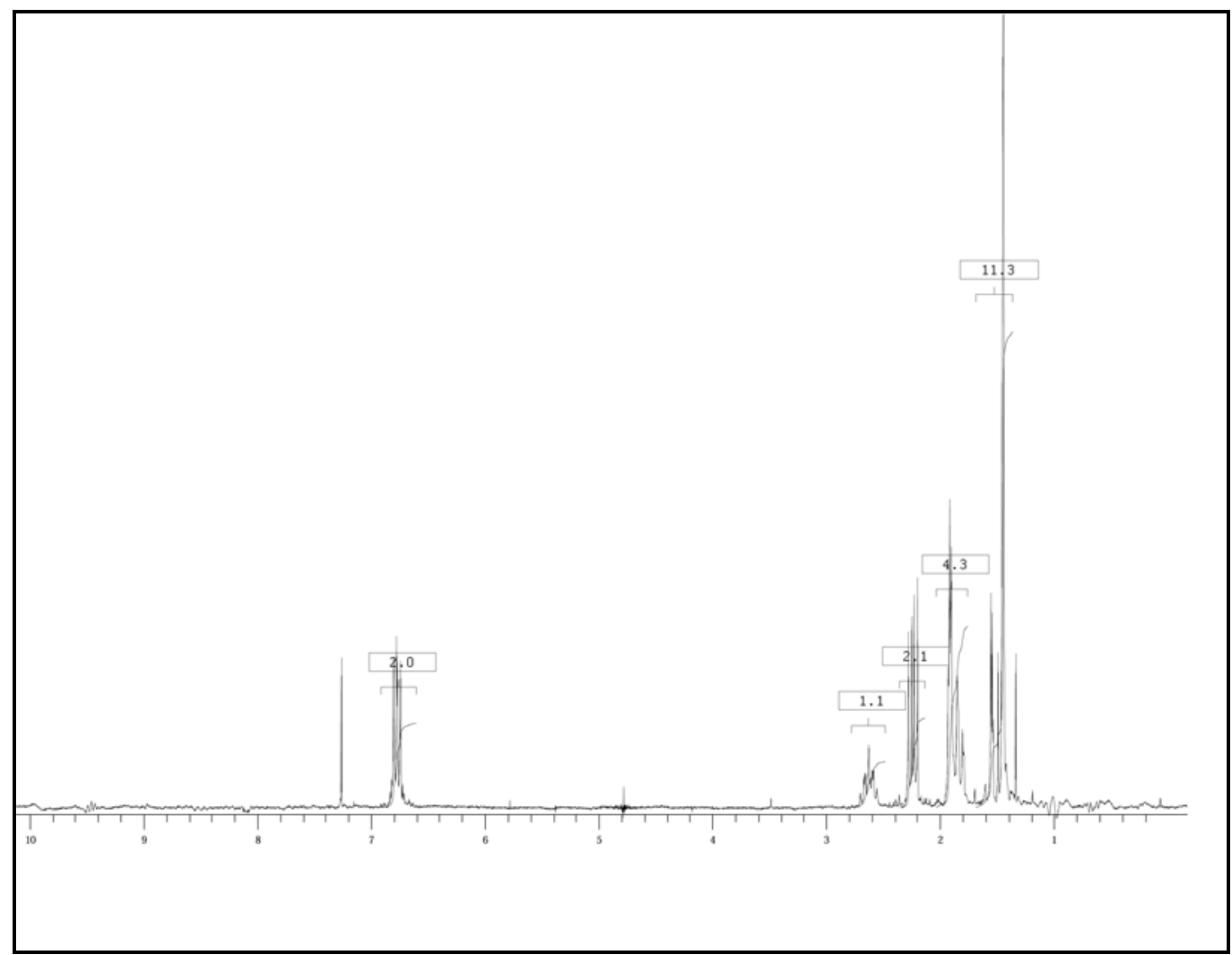
$162 \mathrm{~b}-1$

exo,exo-3-(3,4,5-Trifluorophenyl)bicyclo[3.1.0]hexan-6-carbonsäure-tertbutylester 


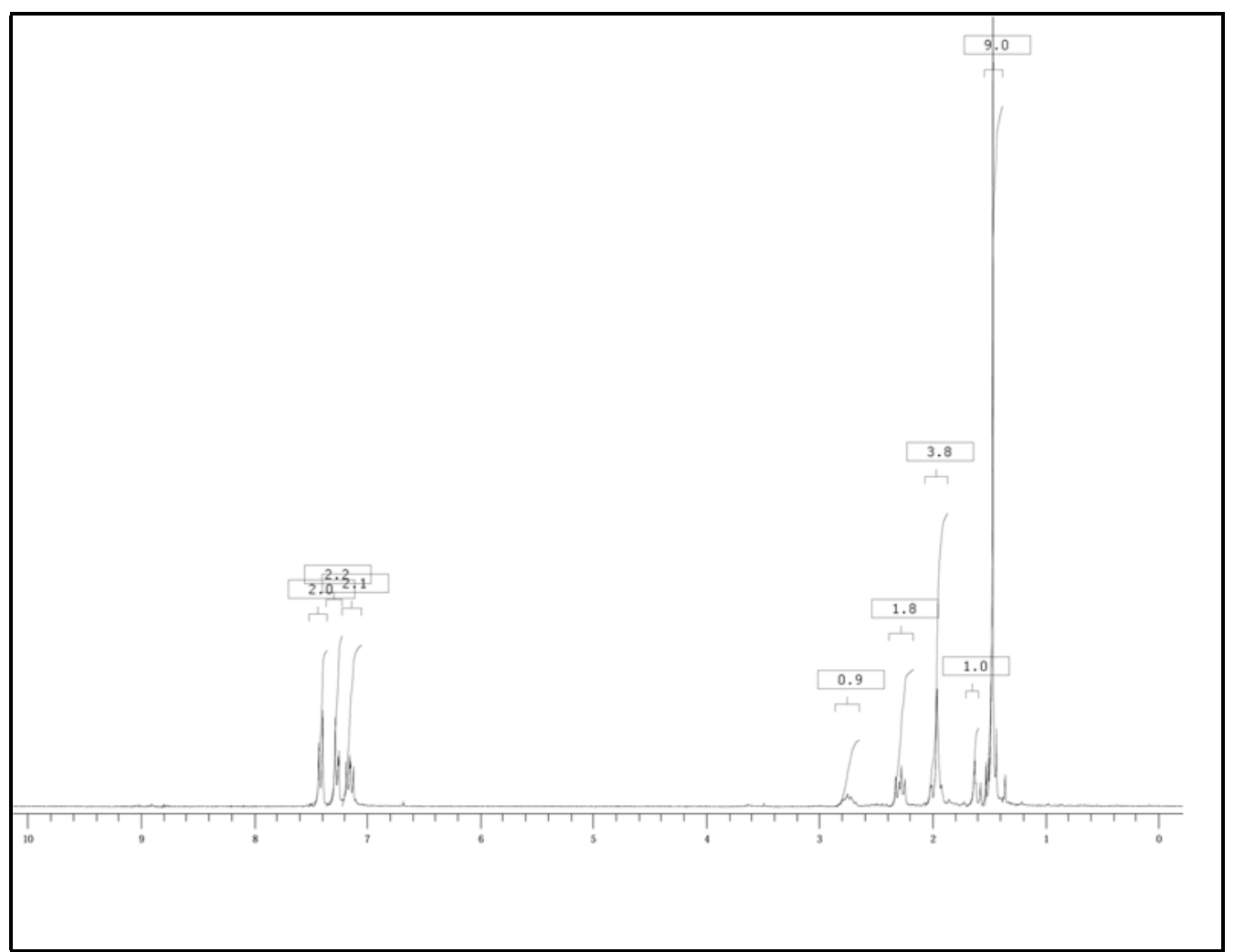

exo,exo-3-[4-(3,4,5-Trifluorophenyl)phenyl]bicyclo[3.1.0]hexan-6-carbonsäuretertbutylester 162c

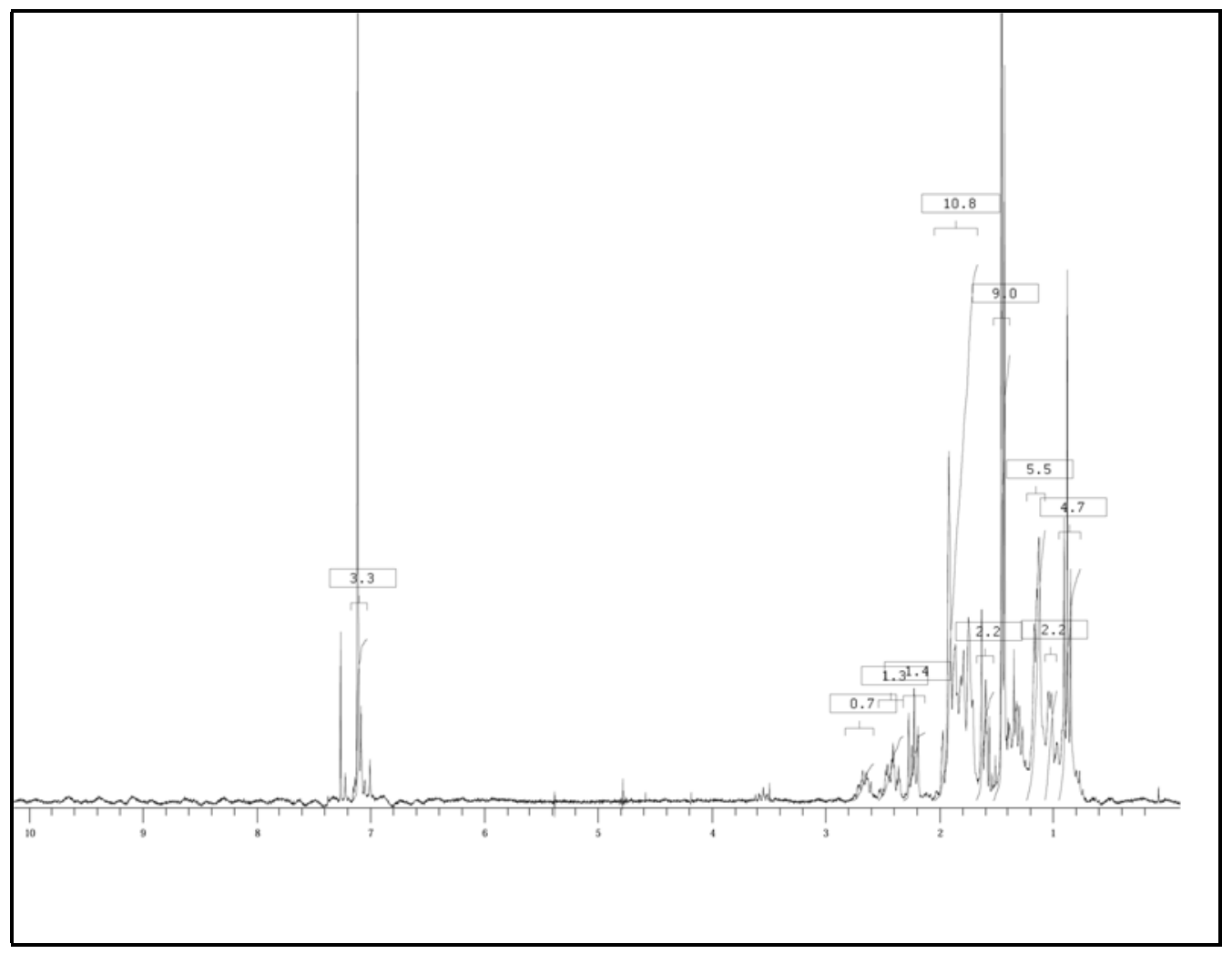

exo,exo-3-[\{trans-4-[(trans-4-n-Propyl)cyclohexyl]cyclohexyl $\}$ phenyl]bicyclo[3.1.0]hexan-6-carbonsäuretertbutylester 162d 


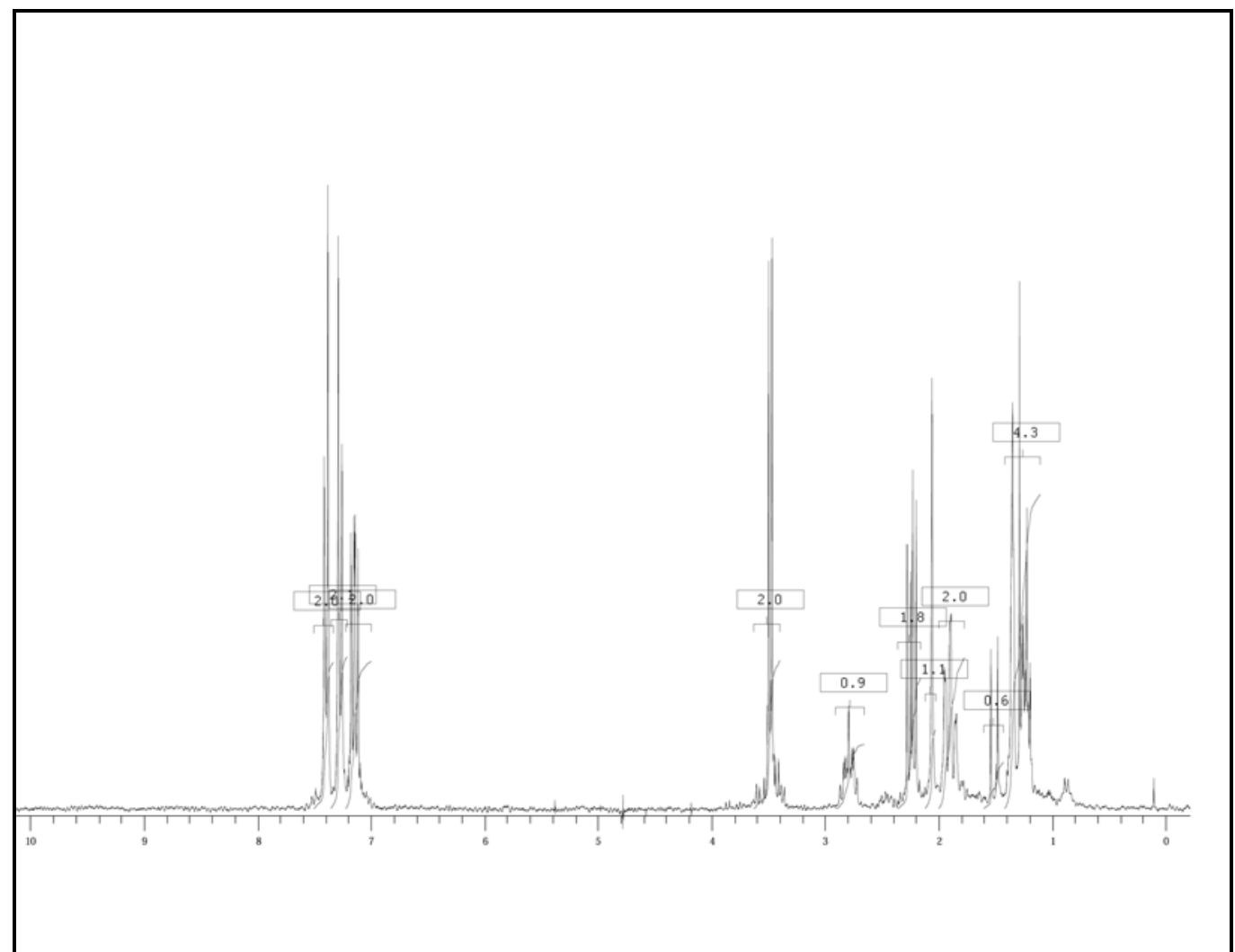

exo,exo-\{3-[4-(3,4,5-Trifluorophenyl)phenyl]bicyclo[3.1.0]hex-6-yl\}methanol 163c

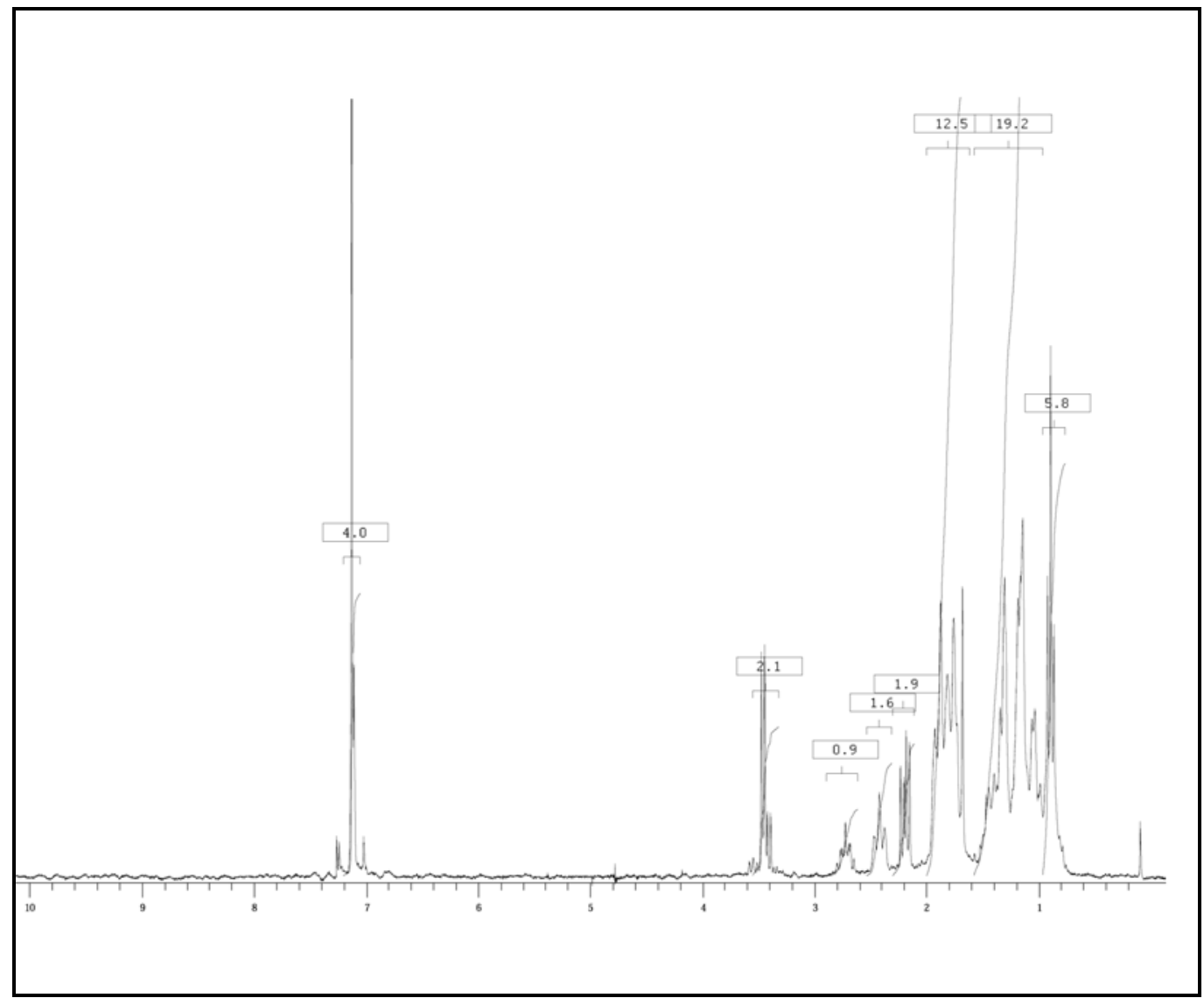

exo,exo-(3-[\{trans-4-[(trans-4-n-Propyl)cyclohexyl]cyclohexyl\}phenyl]bicyclo[3.1.0]hex-6- yl)methanol 163d 


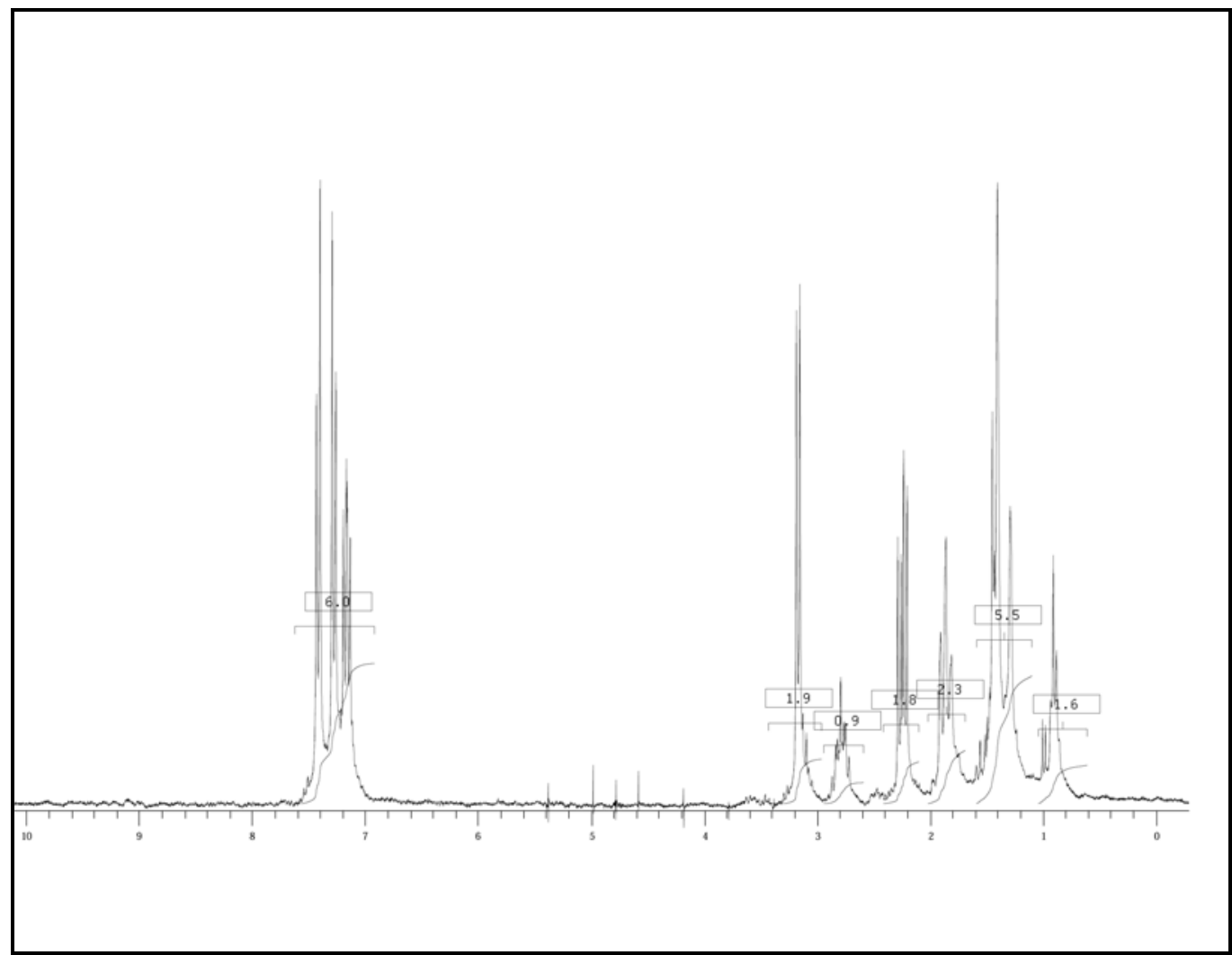

exo,exo-6-(Jodomethyl)-3-[4-(3,4,5-trifluorophenyl)phenyl]bicyclo[3.1.0]hexan 164c

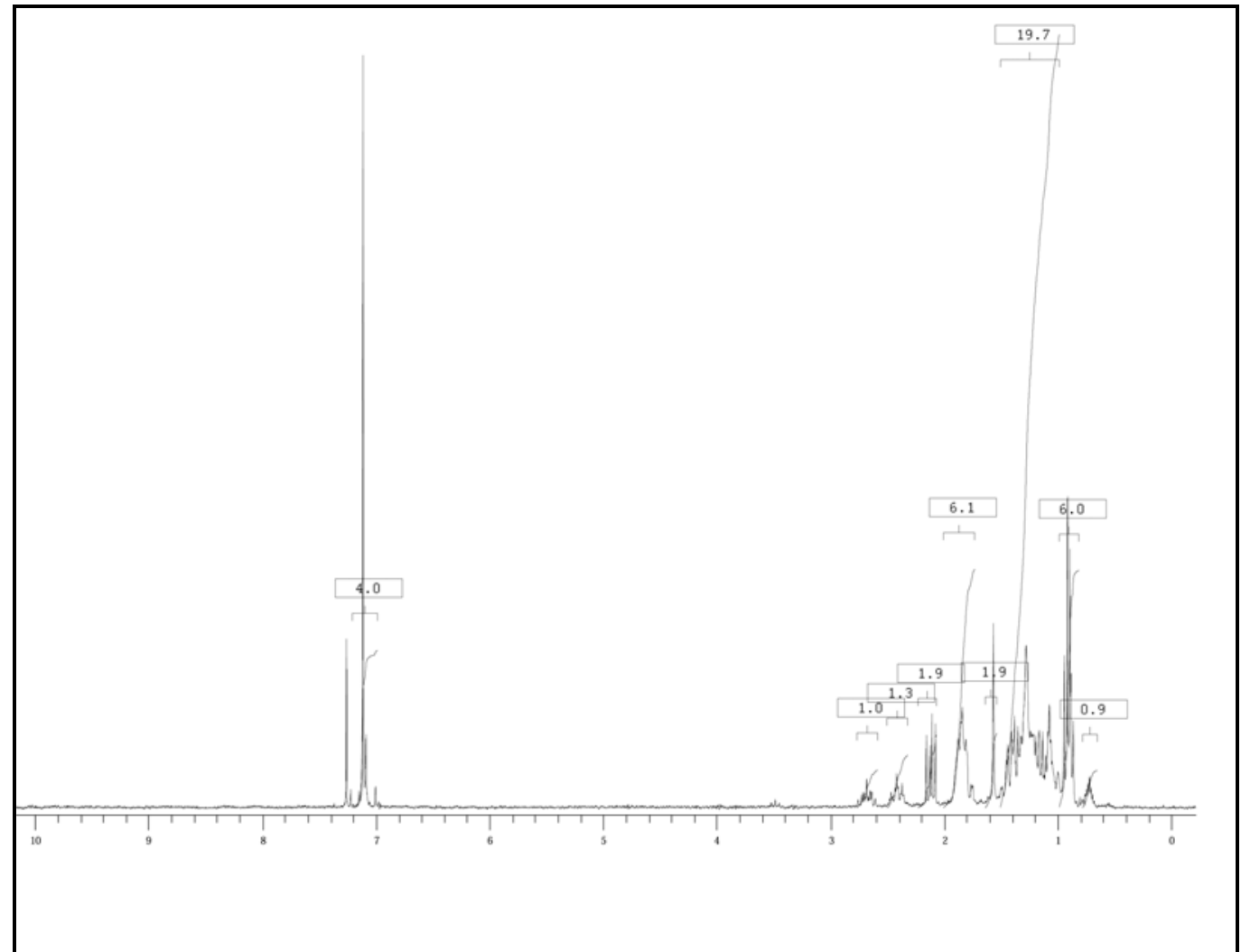

exo,exo-3-\{[4-(trans-4-n-Pentyl)cyclohexyl]phenyl $\}-6-n$ -

propylbicyclo[3.1.0]hexan 165a 


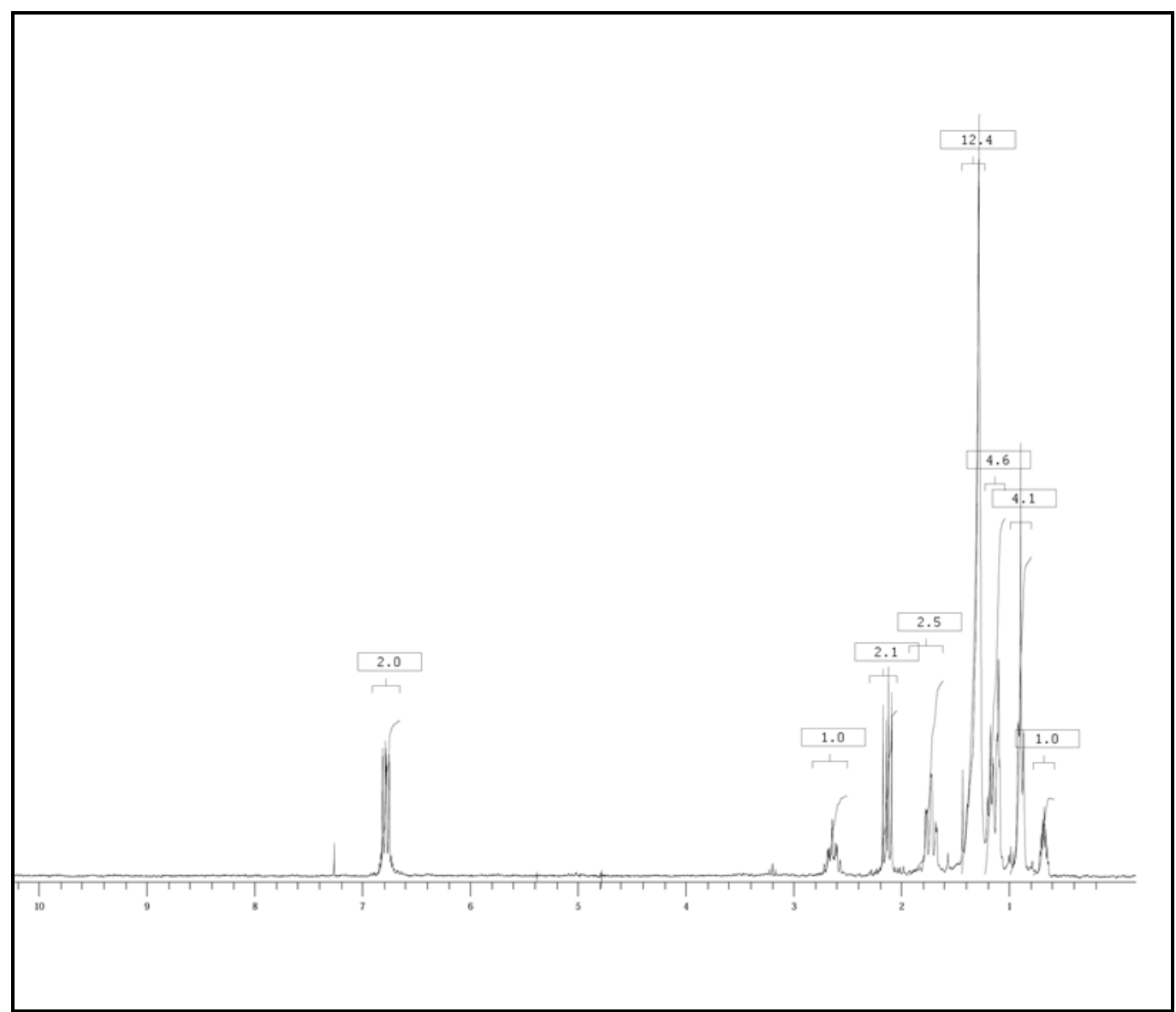

exo,exo-6-n-Heptyl-3-(3,4,5-trifluorophenyl)bicyclo[3.1.0]hexan 165b

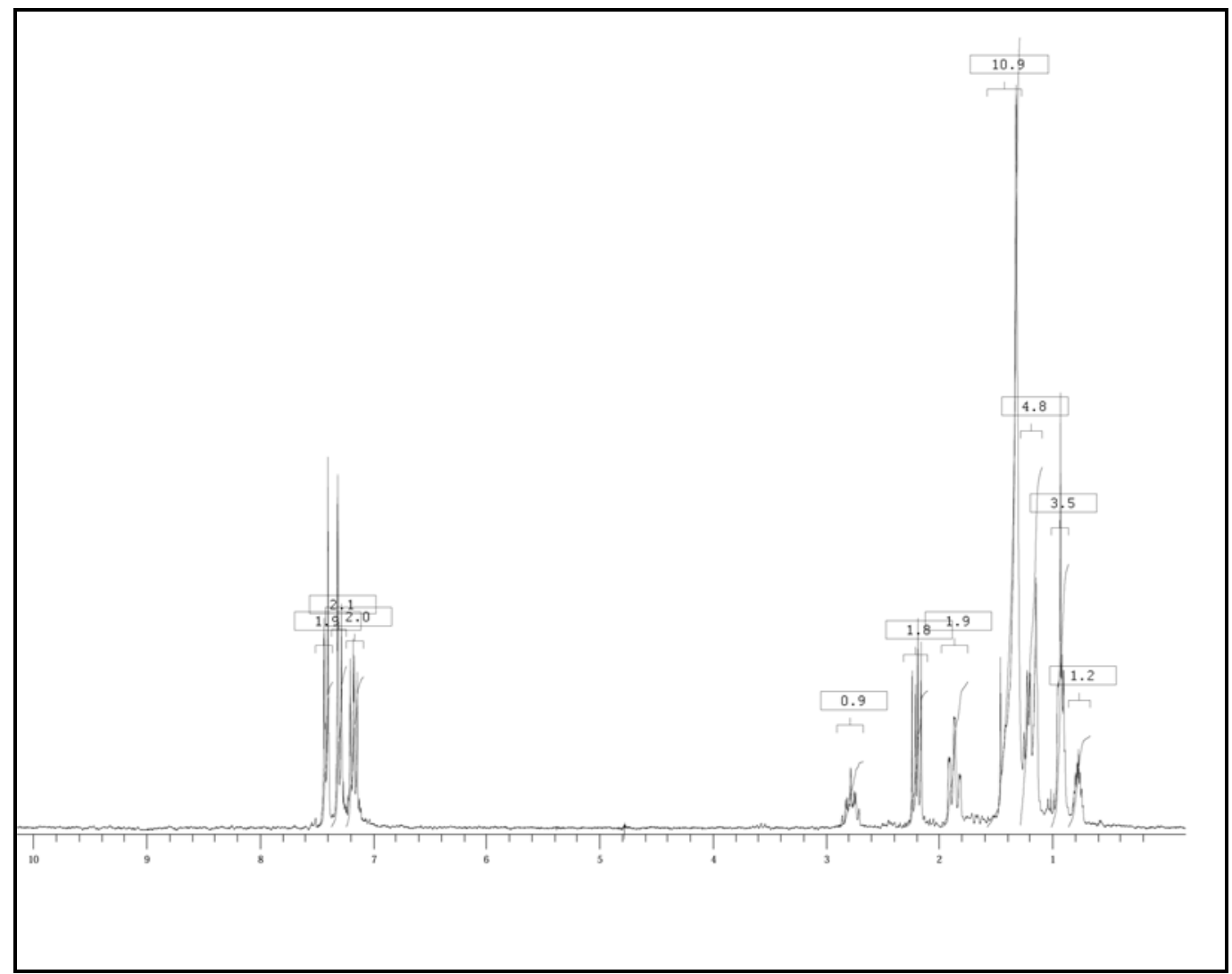

exo,exo-6-n-Heptyl-3-[4-(3,4,5-trifluorophenyl)phenyl]bicyclo[3.1.0]hexan 165c 


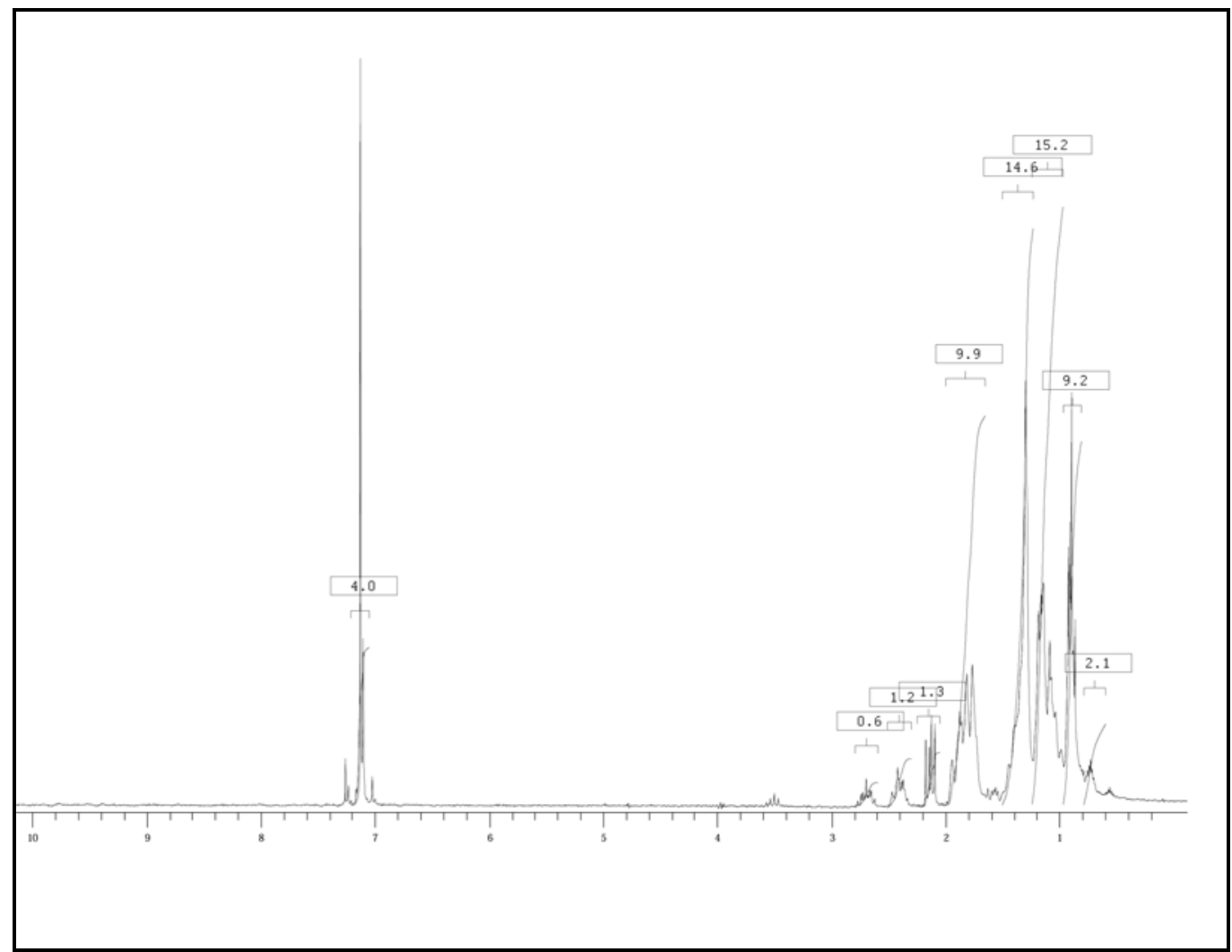

exo,exo-6-n-Heptyl-3-[\{trans-4-[(trans-4-n-

propyl)cyclohexyl]cyclohexyl $\}$ phenyl]bicyclo-[3.1.0]hexan $\mathbf{1 6 5 d}$ 


\section{2. ${ }^{13}$ C-NMR-Spektren}

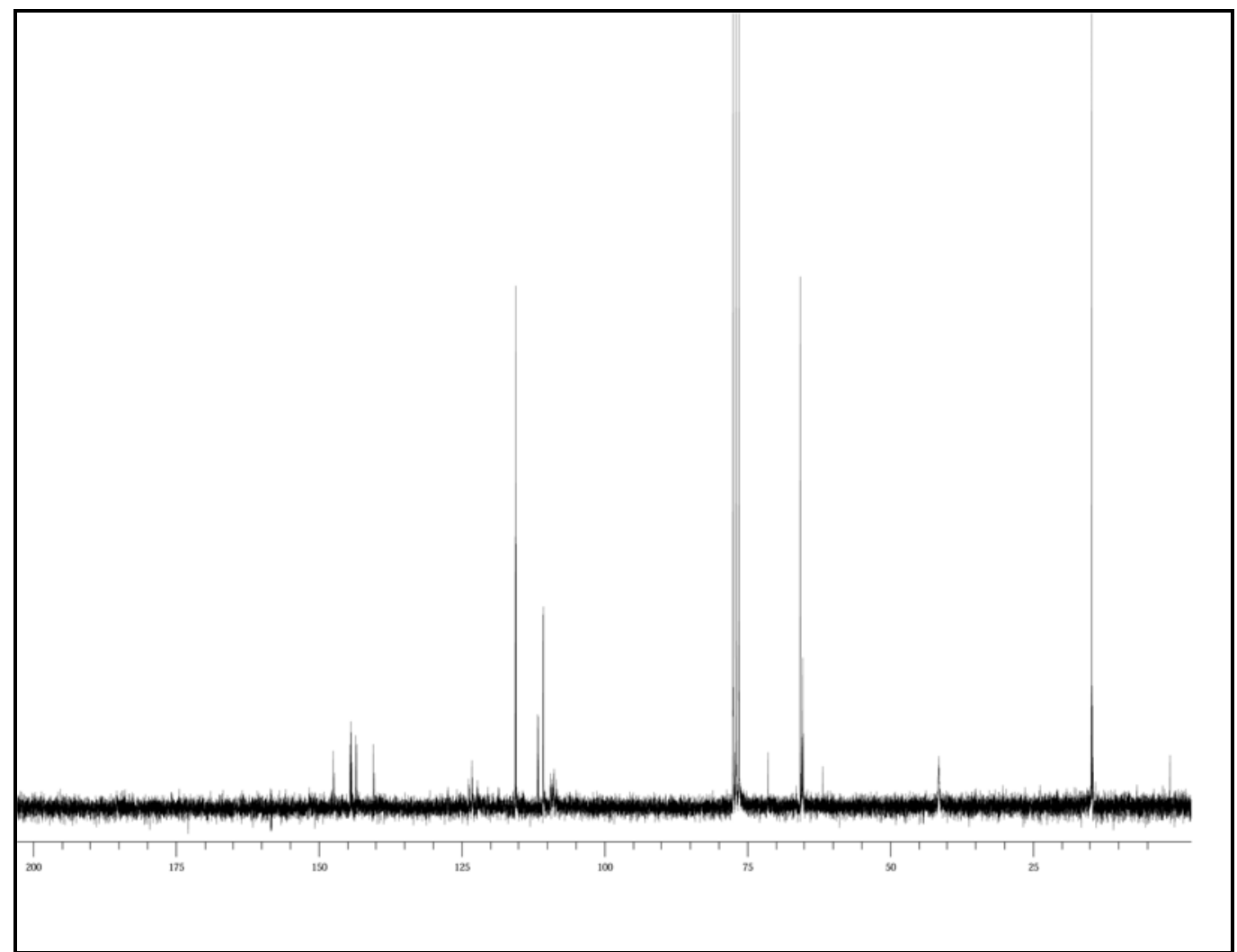

exo,exo-Bicyclo[3.1.0]hexan-3,6-dicarbonsäure-3-tbutylester-6-ethylester 33a

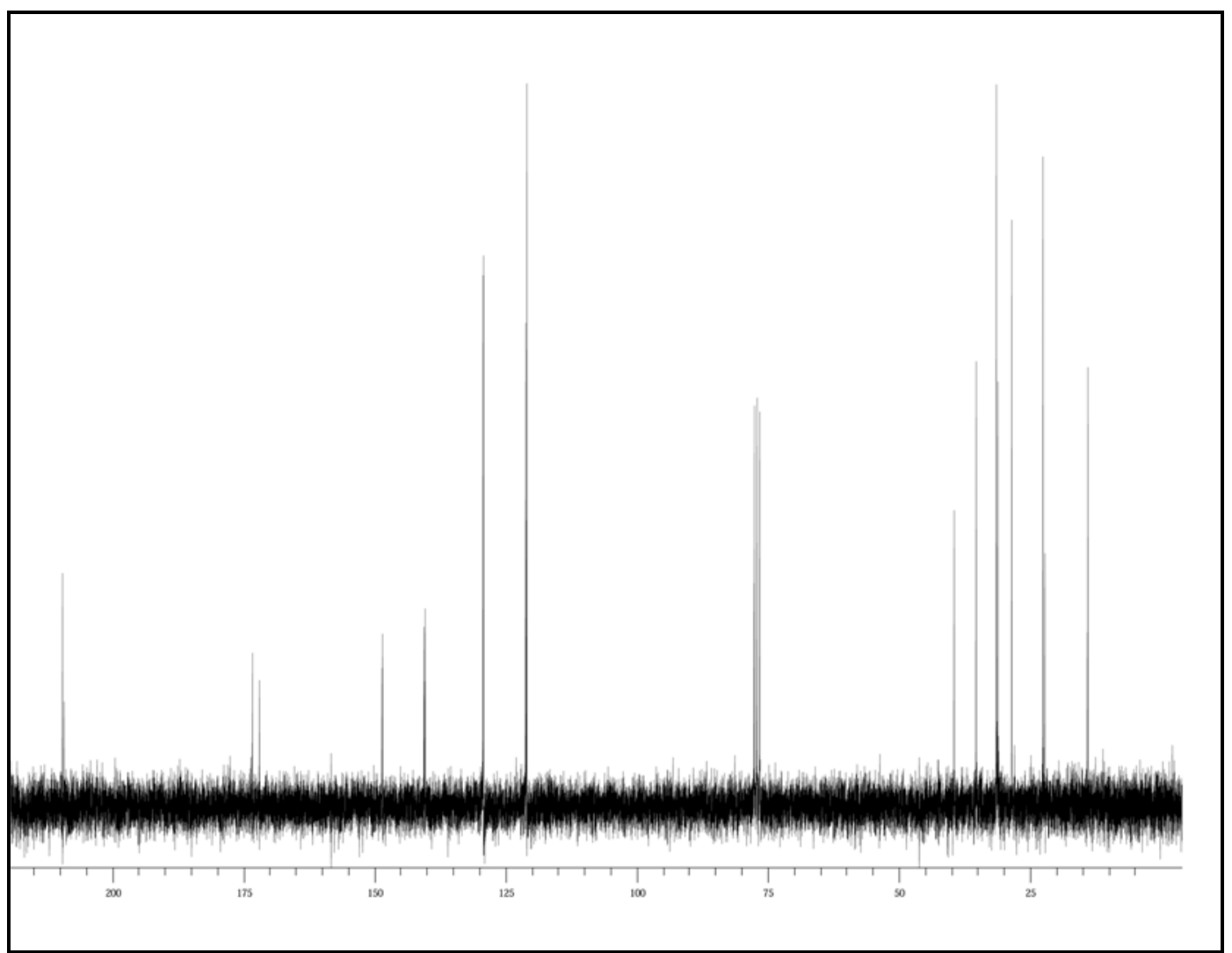

exo,exo-Bicyclo[3.1.0]hexan-3,6-dicarbonsäure-3,6-di(4-npentylphenyl)ester 35a 


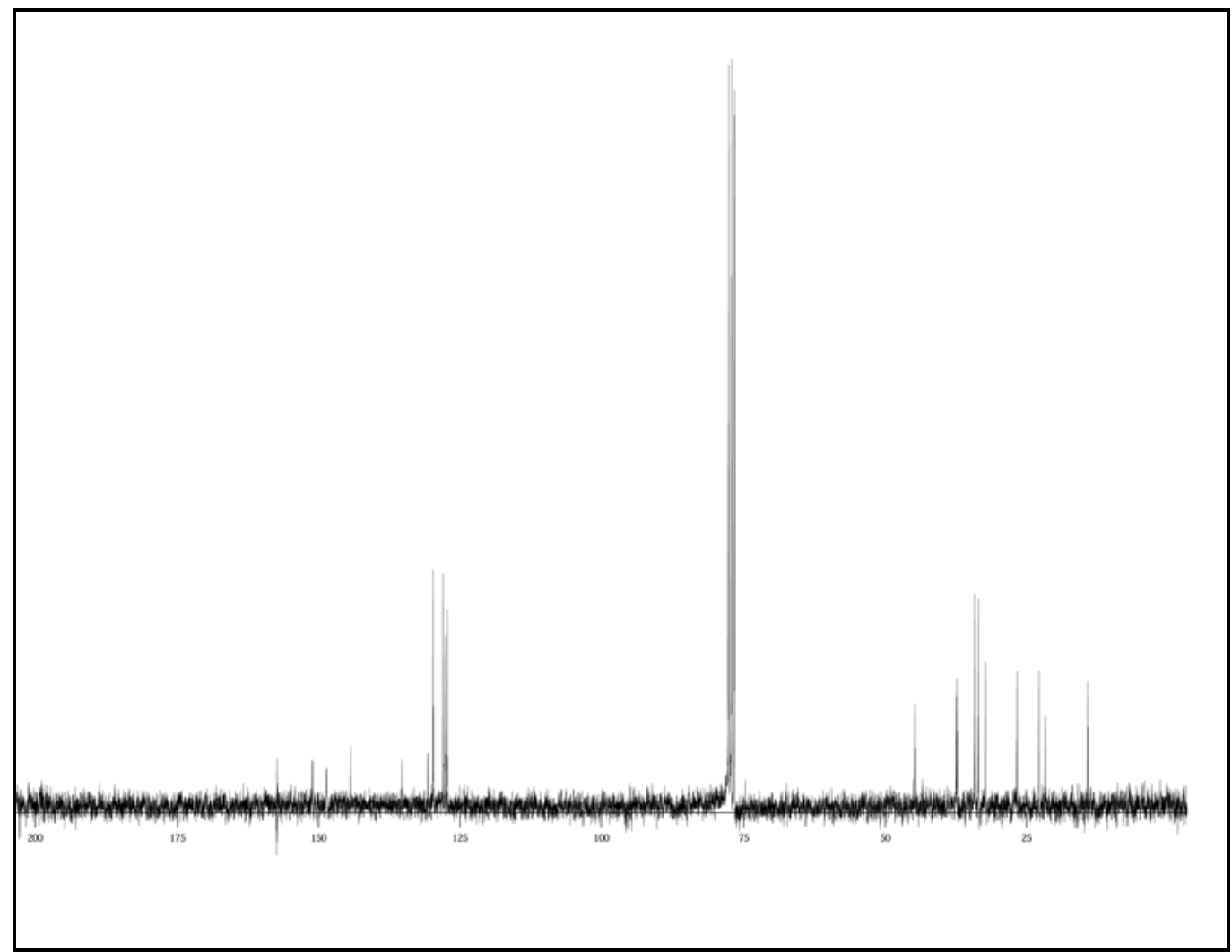

1-(Cyclopenten-4-yl)-4-[(trans-4-n-pentyl)cyclohexyl]benzol 89

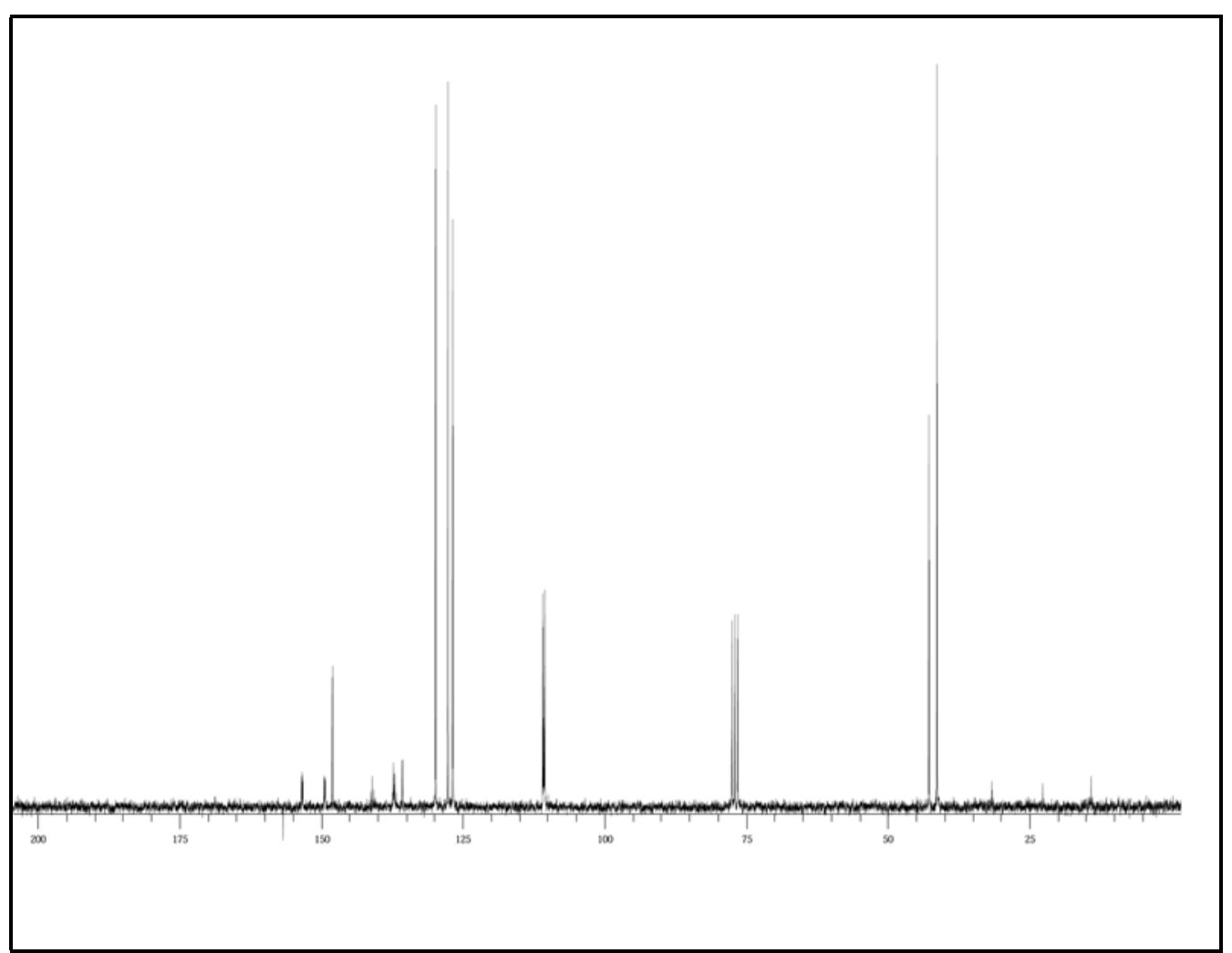

4-[4-(3,4,5-Trifluorophenyl)phenyl]cyclopenten 90 


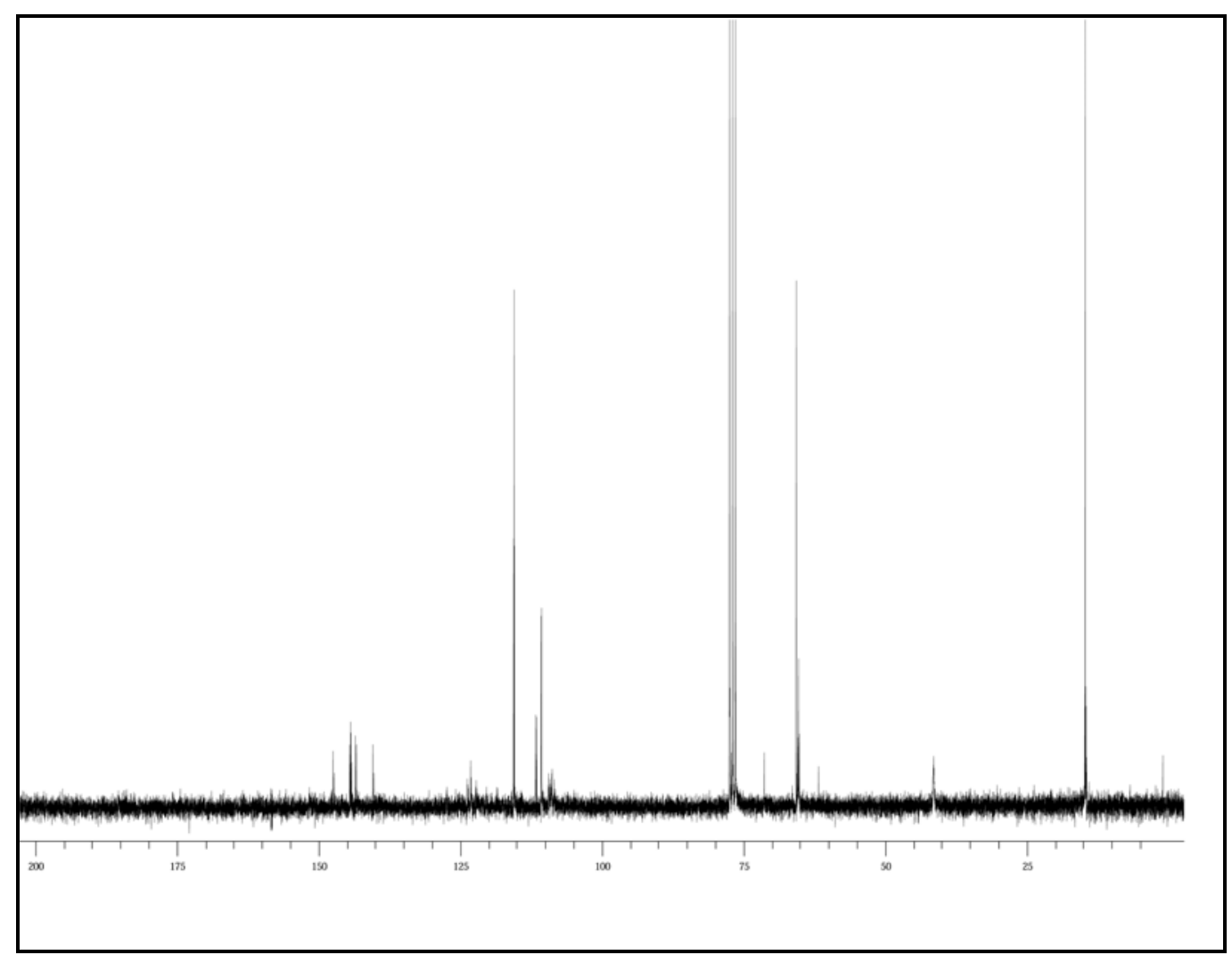

4-Ethoxy-2,3-difluorphenyldiazomethan 143b

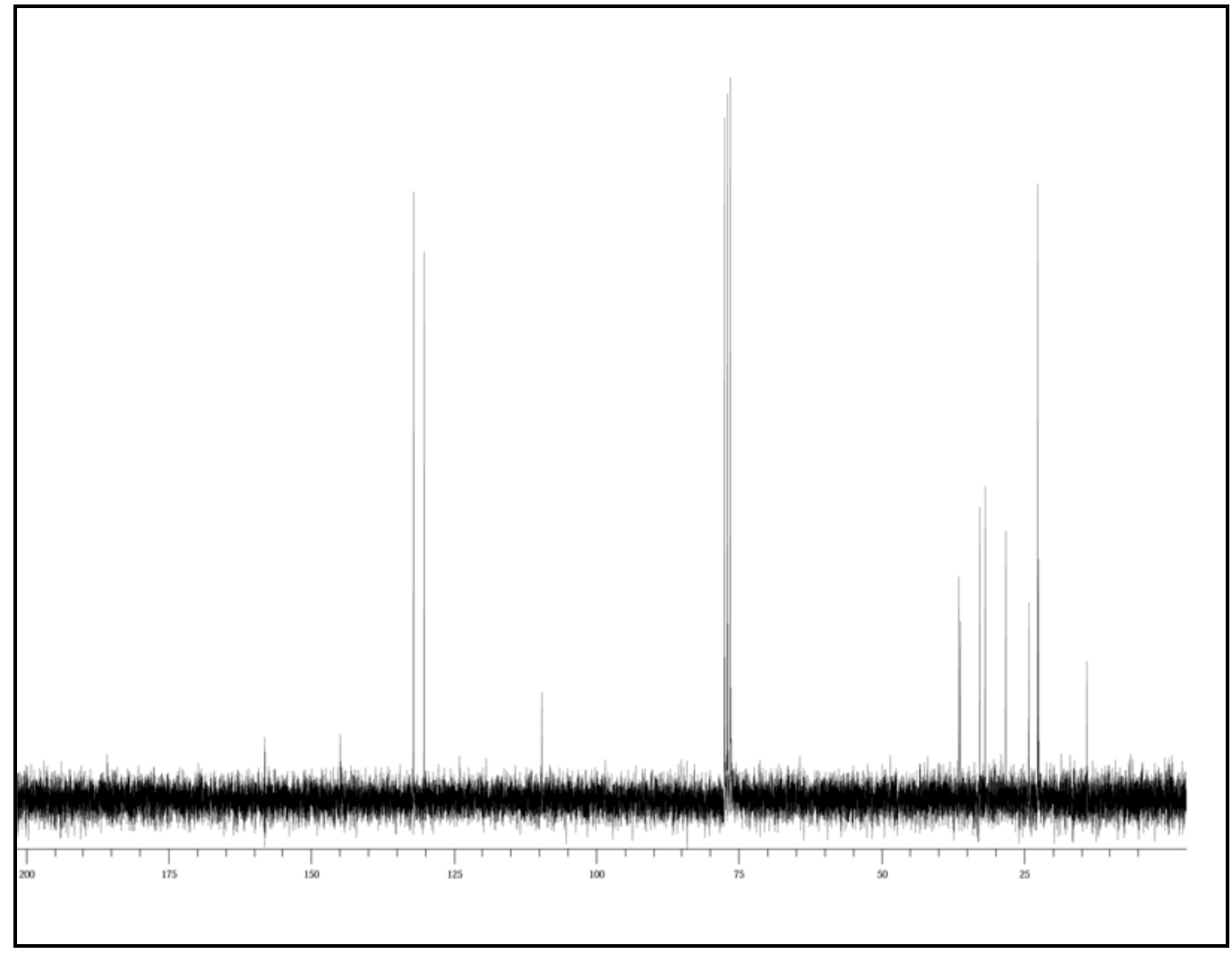

exo,exo-6-(4-Cyanophenyl)-3-npentylbicyclo[3.1.0]hexan 155a 


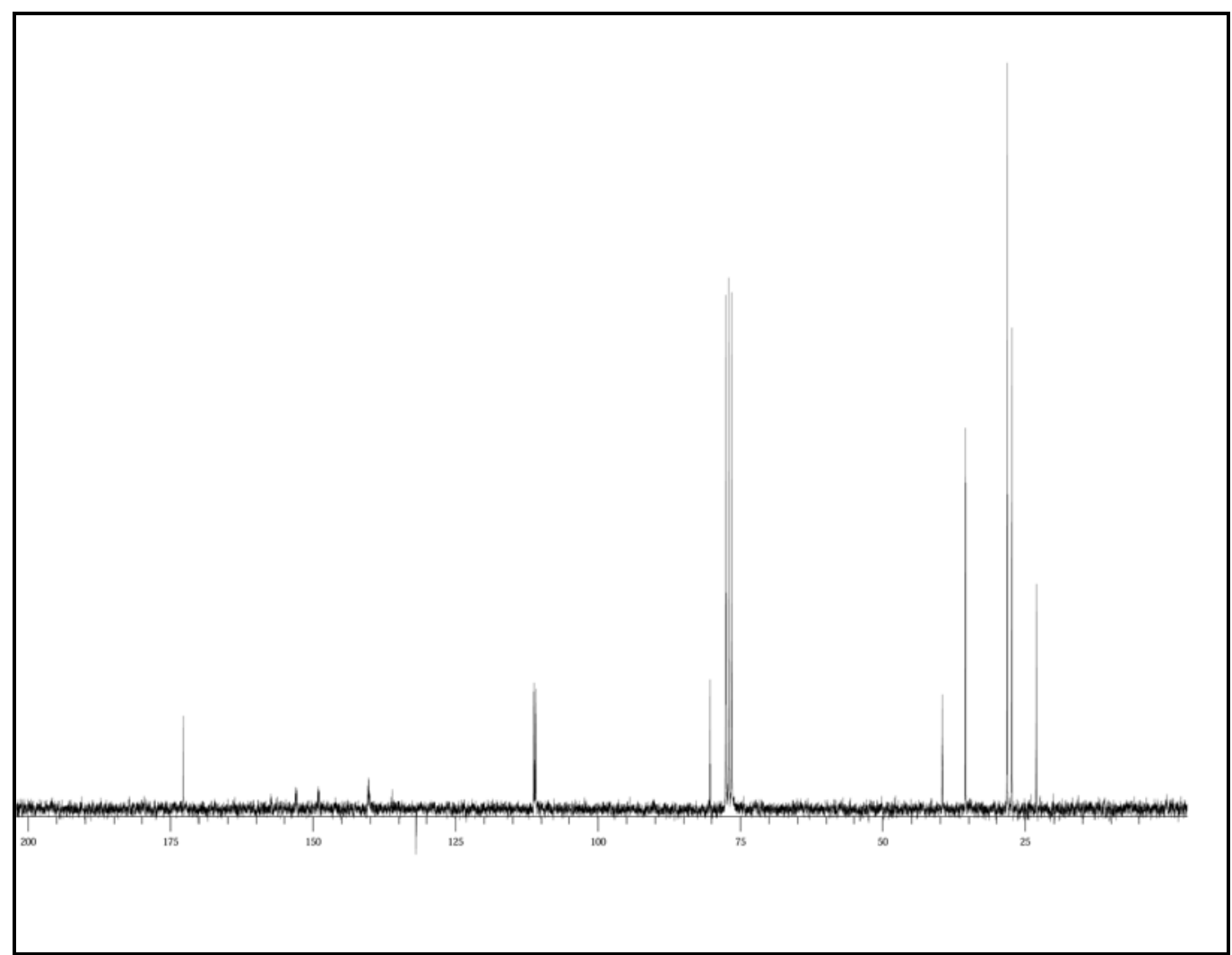

exo,exo-3-(3,4,5-Trifluorophenyl)bicyclo[3.1.0]hexan-6-carbonsäure-tertbutylester 162b

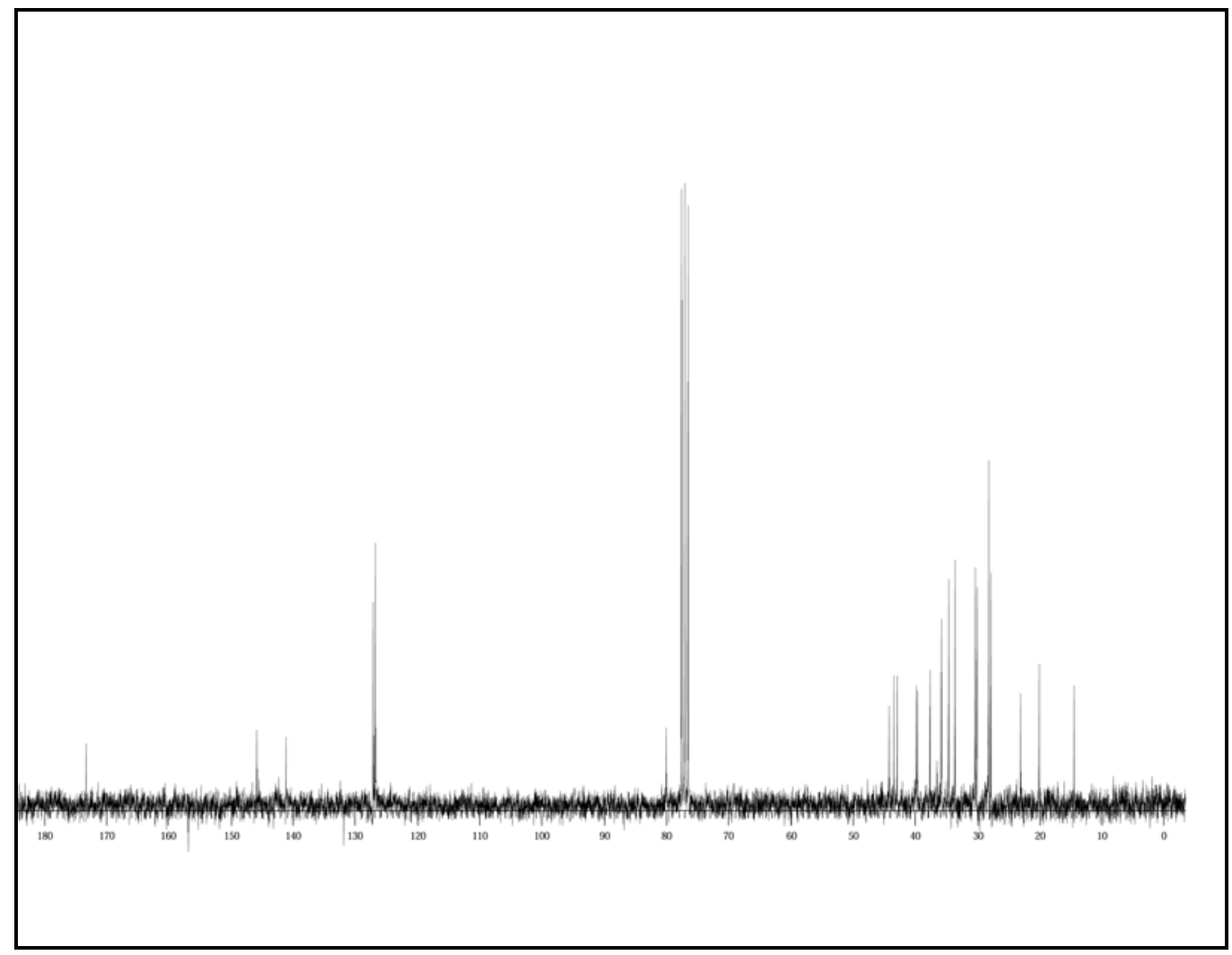

exo,exo-3-[\{trans-4-[(trans-4-n-Propyl)cyclohexyl]cyclohexyl\} phenyl]bicyclo[3.1.0]hexan-6-carbonsäuretertbutylester 162d 


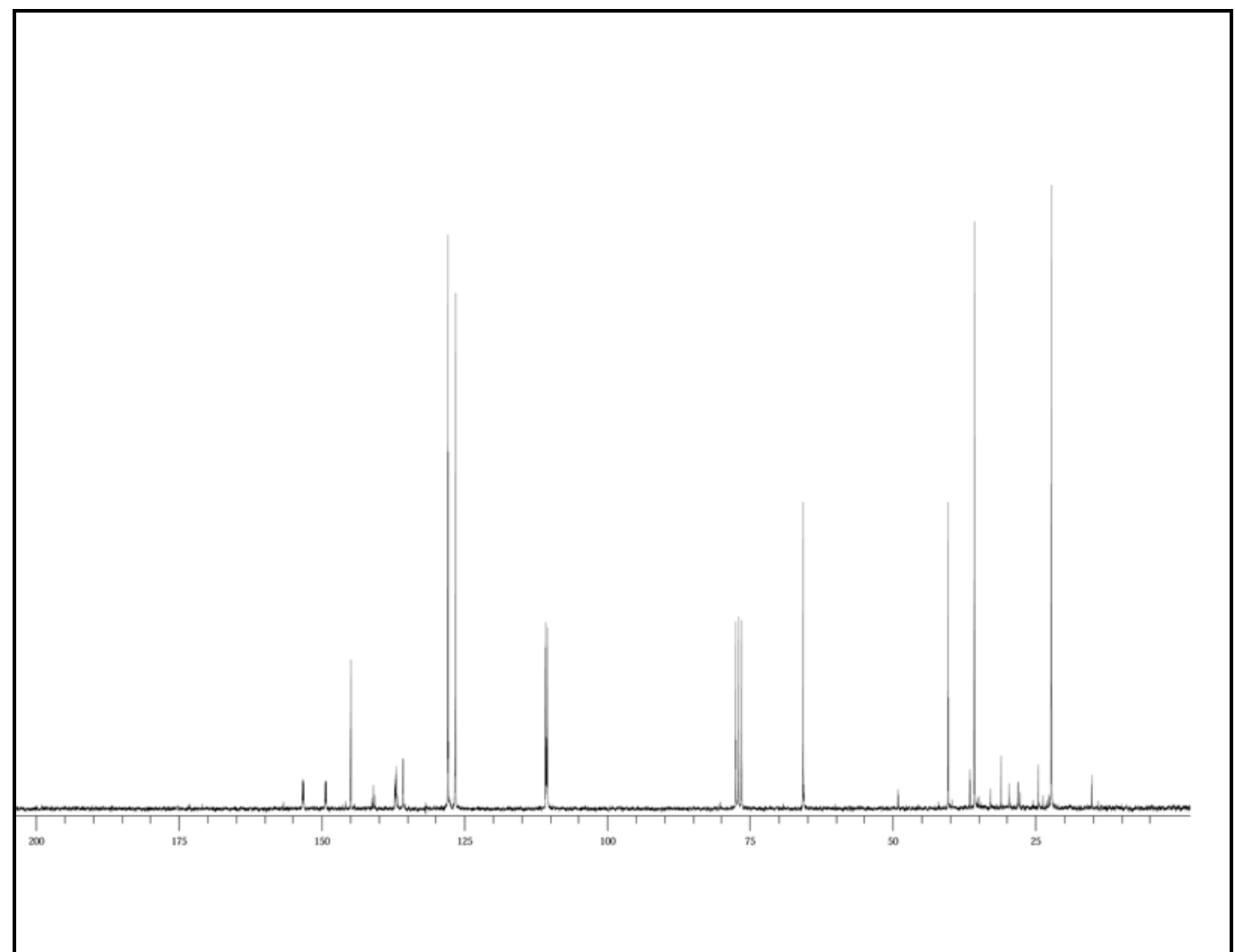

exo,exo-\{3-[4-(3,4,5-Trifluorophenyl)phenyl]bicyclo[3.1.0]hex-6-yl\}methanol 163c

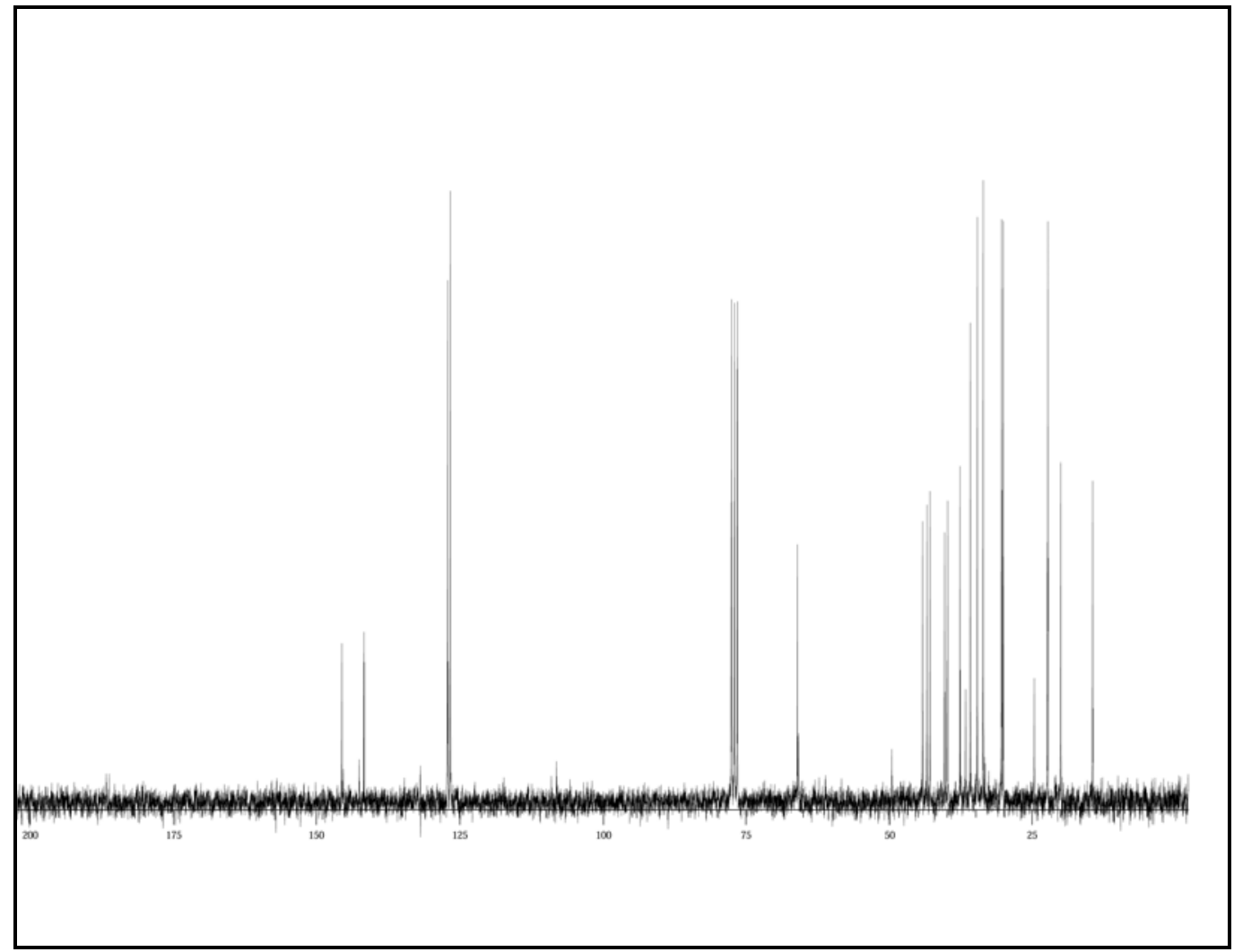

exo,exo-(3-[\{trans-4-[(trans-4- $n$-Propyl)cyclohexyl]cyclohexyl\} phenyl]bicyclo[3.1.0]hex-6- yl)methanol 163d 


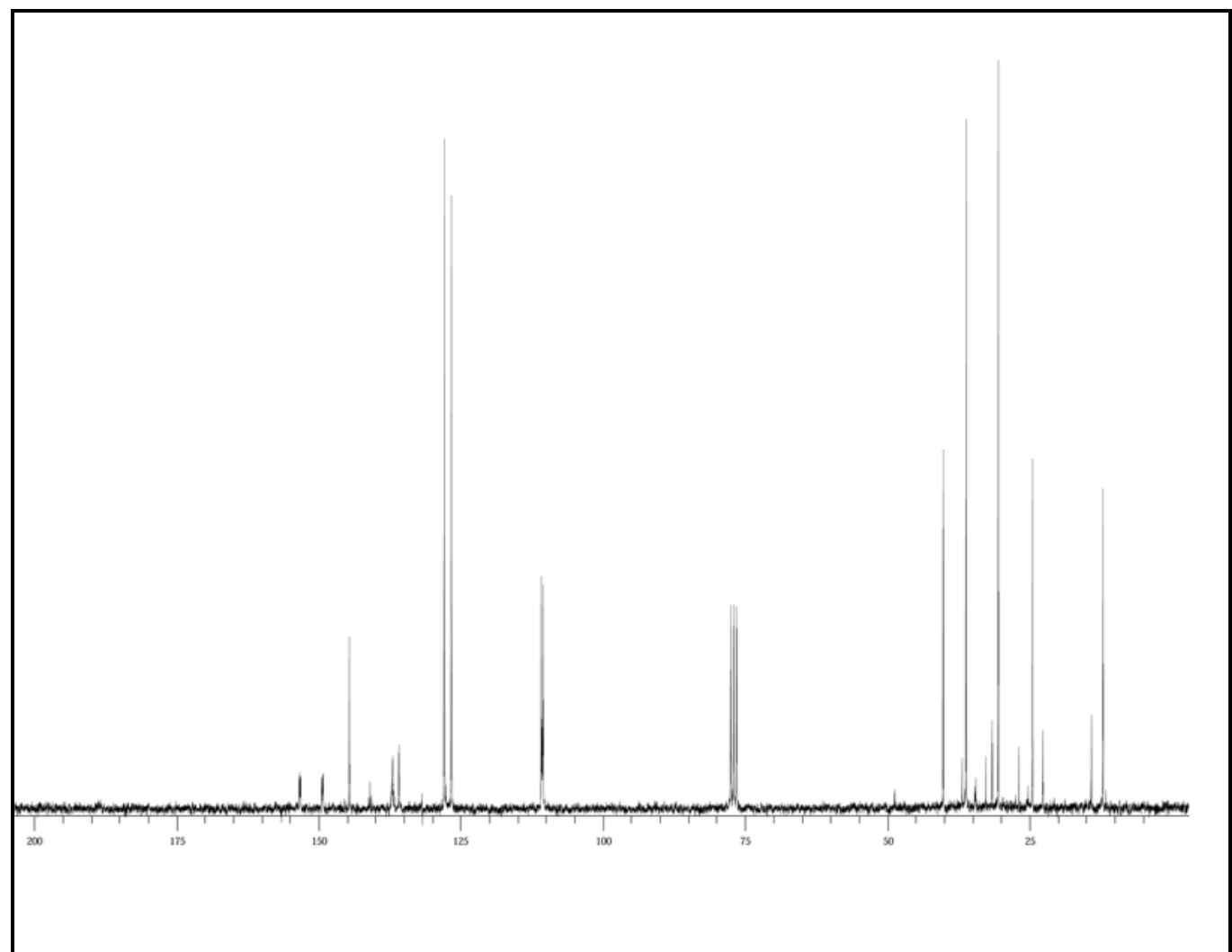

exo,exo-6-(Jodomethyl)-3-[4-(3,4,5-trifluorophenyl)phenyl]bicyclo[3.1.0]hexan 164c

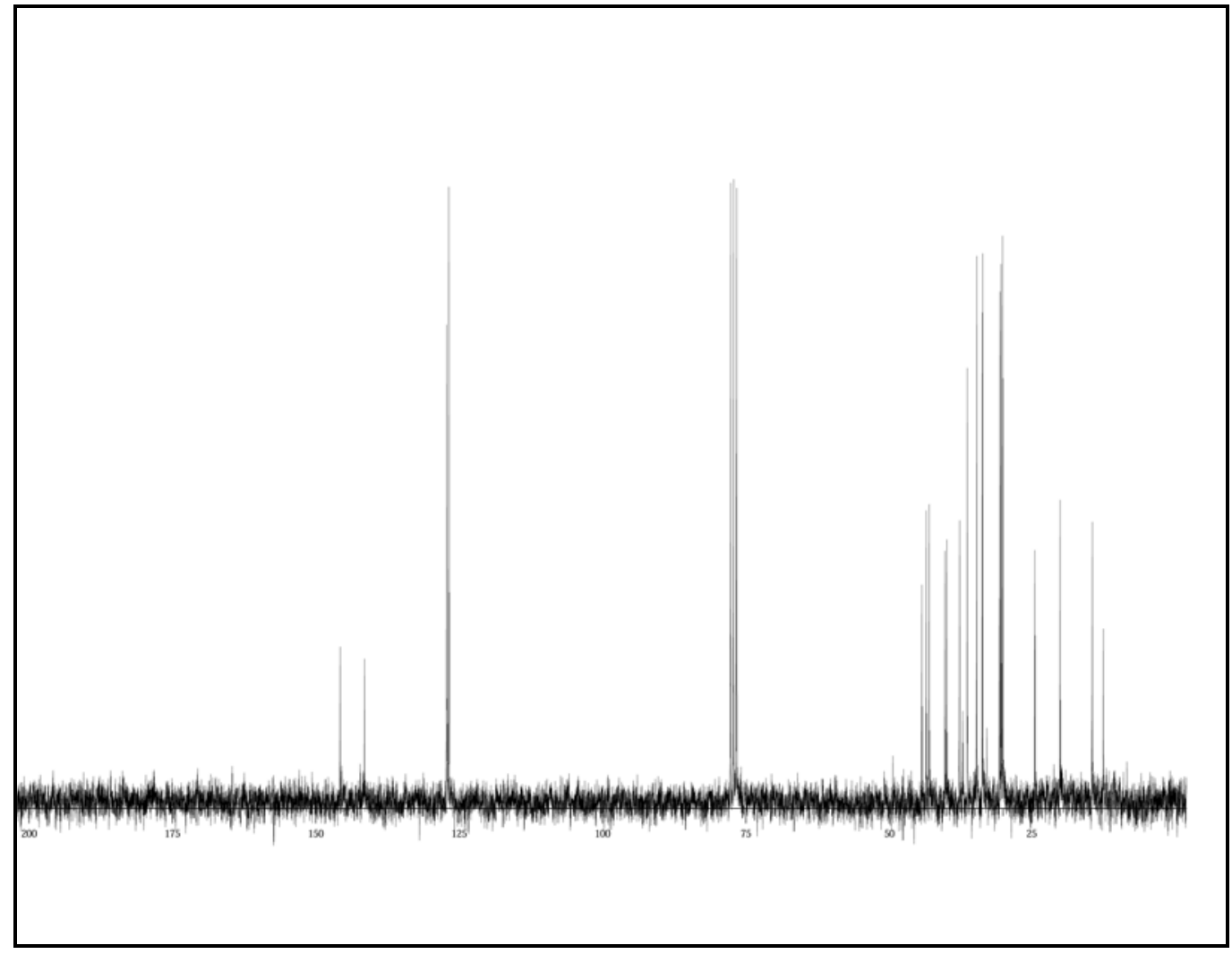

exo,exo-6-(Jodomethyl)-3-[\{trans-4-[(trans-4- $n$ -

propyl)cyclohexyl]cyclohexyl\} phenyl]- bicyclo[3.1.0]hexan 164d 


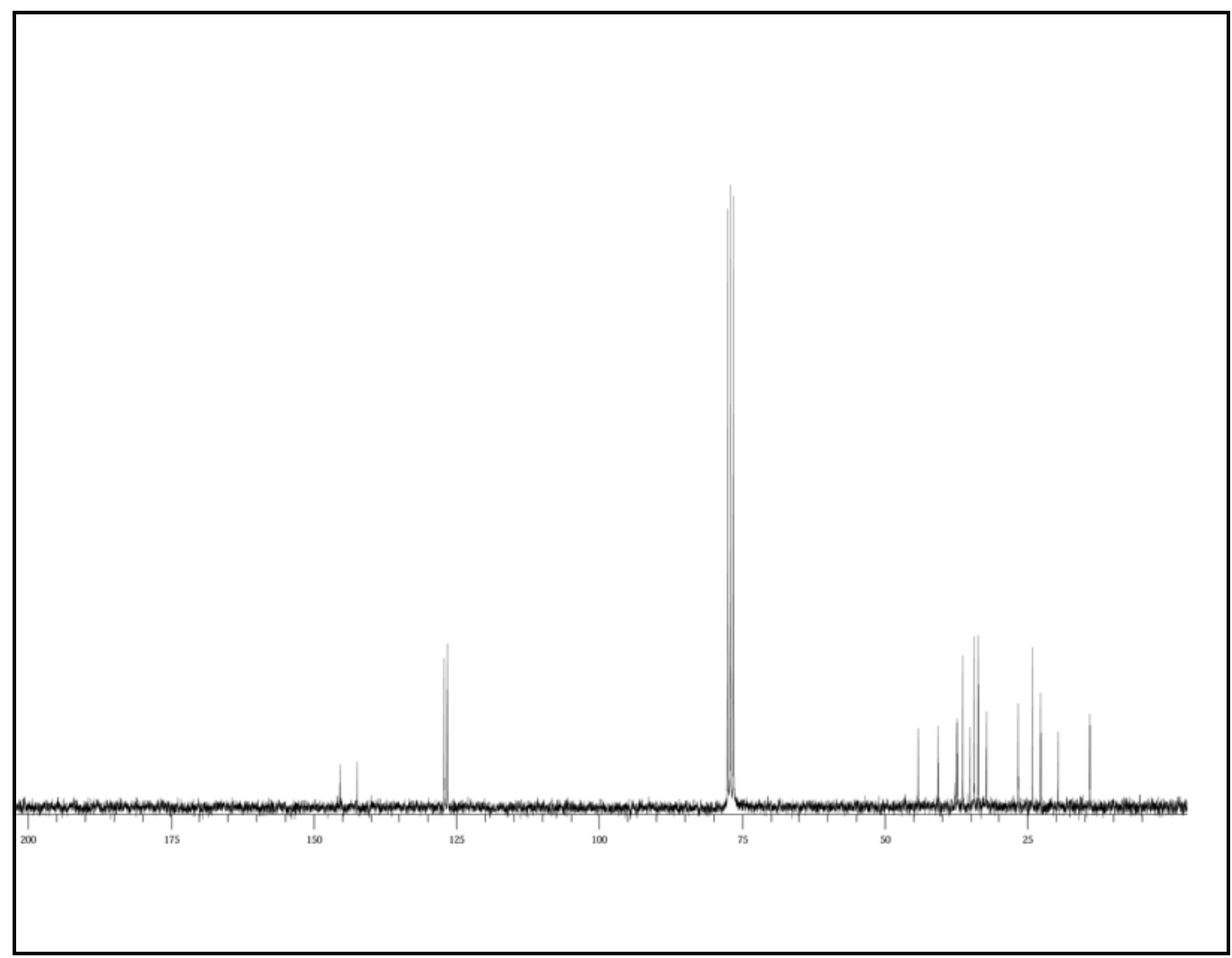

exo,exo-3-\{[4-(trans-4- $n$-Pentyl)cyclohexyl]phenyl\}-6- $n$-propylbicyclo[3.1.0]hexan 165a

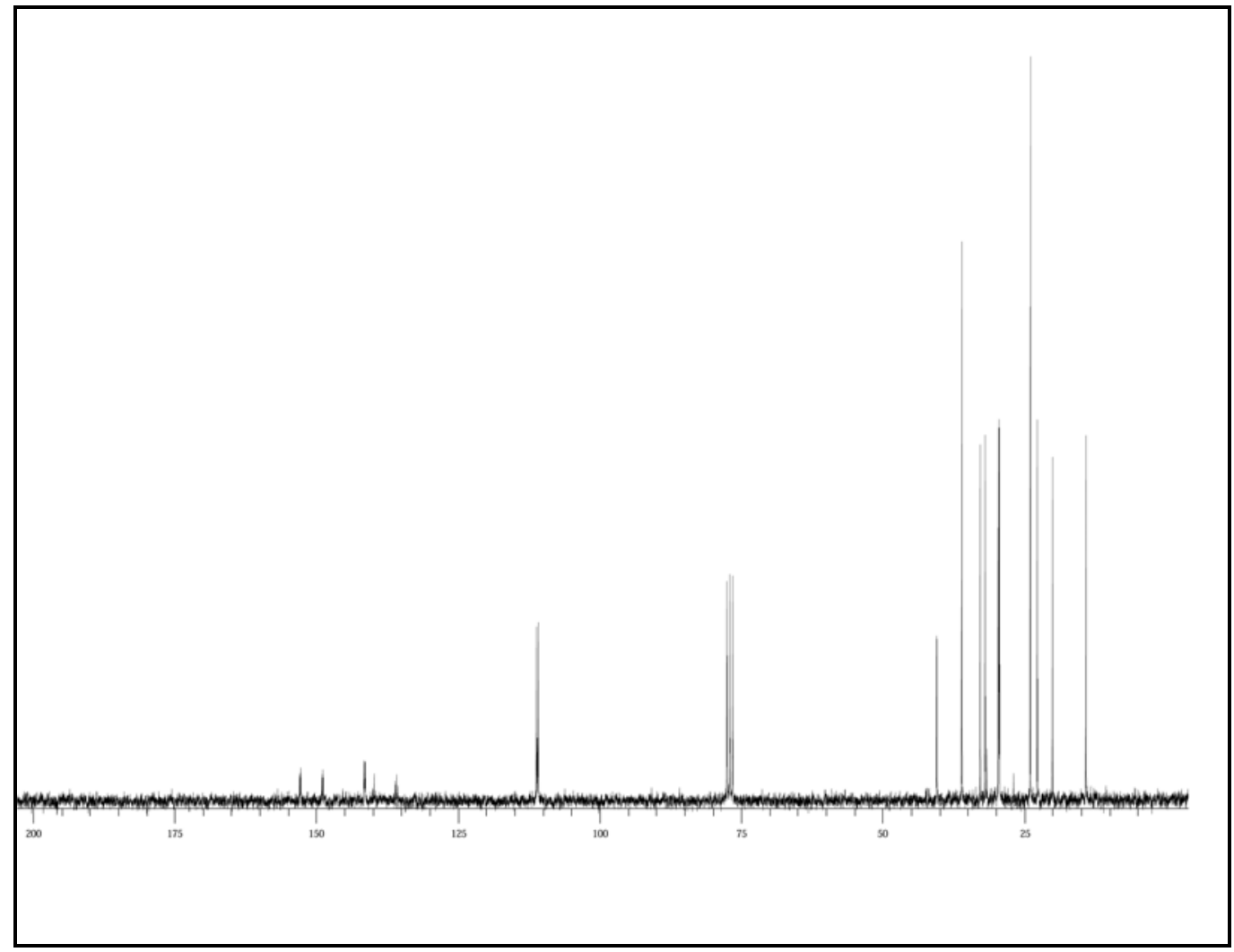

exo,exo-6-n-Heptyl-3-(3,4,5-trifluorophenyl)bicyclo[3.1.0]hexan 165b 


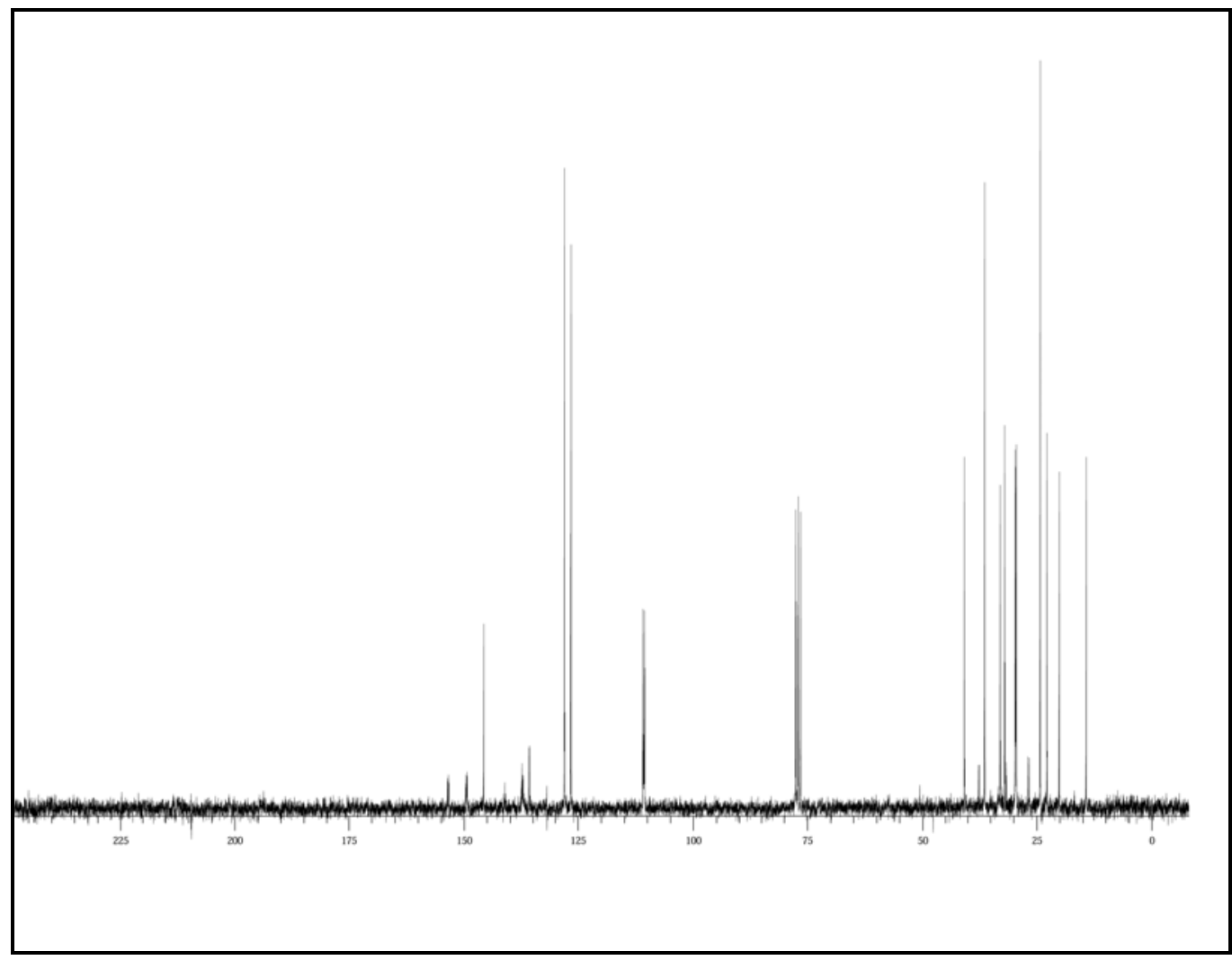

exo,exo-6-n-Heptyl-3-[4-(3,4,5-trifluorophenyl)phenyl]bicyclo[3.1.0]hexan 165c

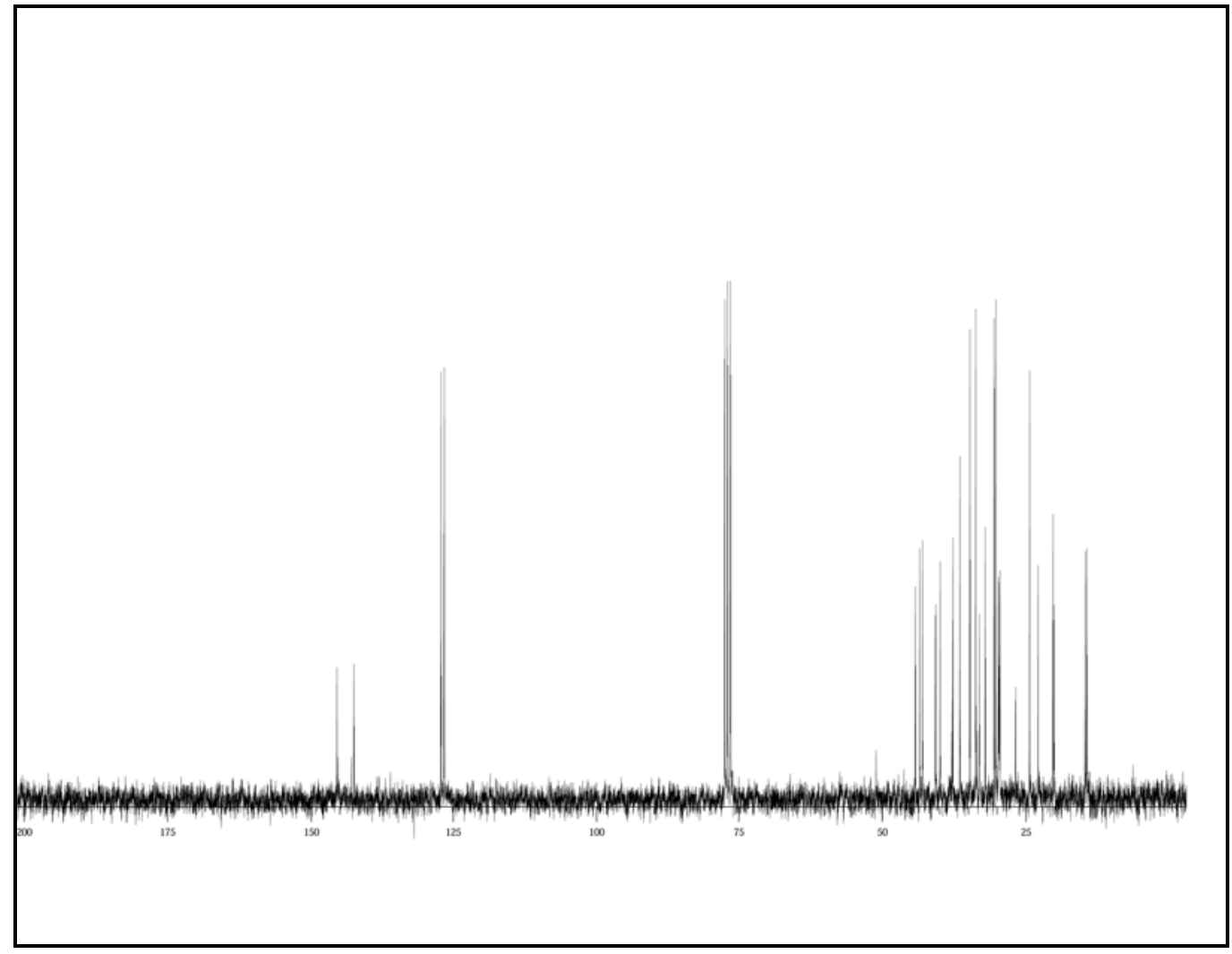

exo,exo-6-n-Heptyl-3-[\{trans-4-[(trans-4-n-propyl)cyclohexyl]cyclohexyl $\}$ phenyl]bicyclo-[3.1.0]hexan 165d 
3. Differential Scanning Calometry (DSC)

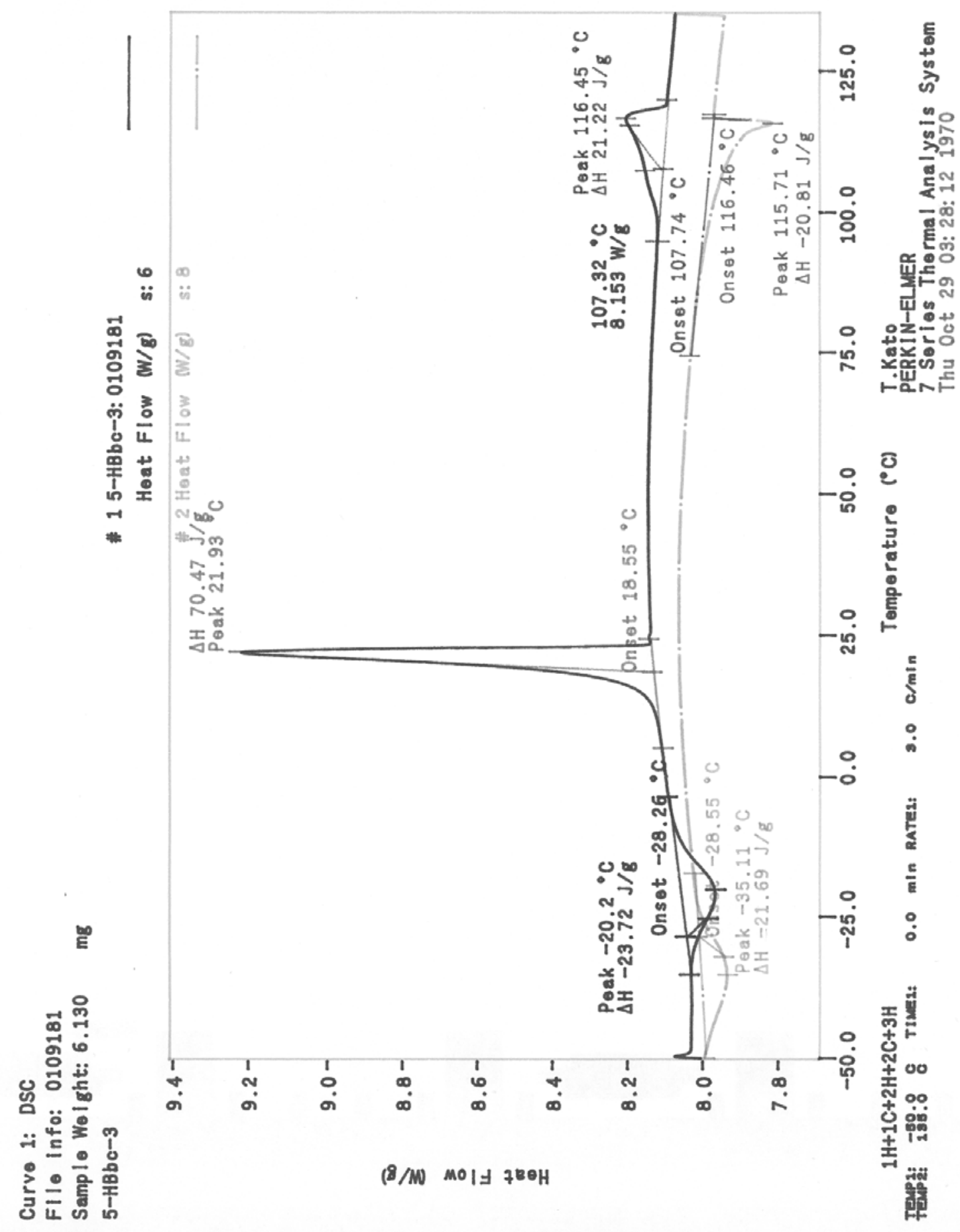




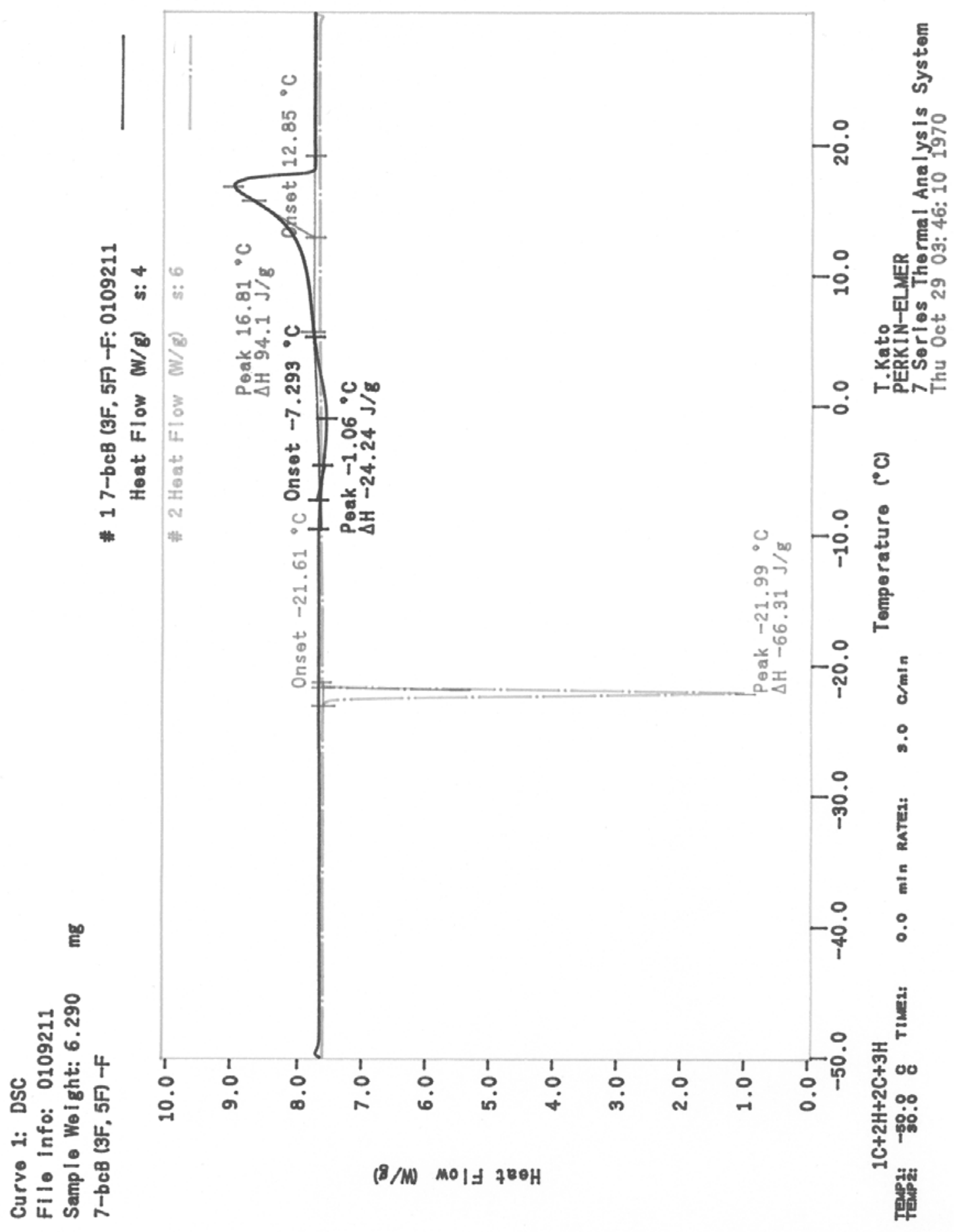




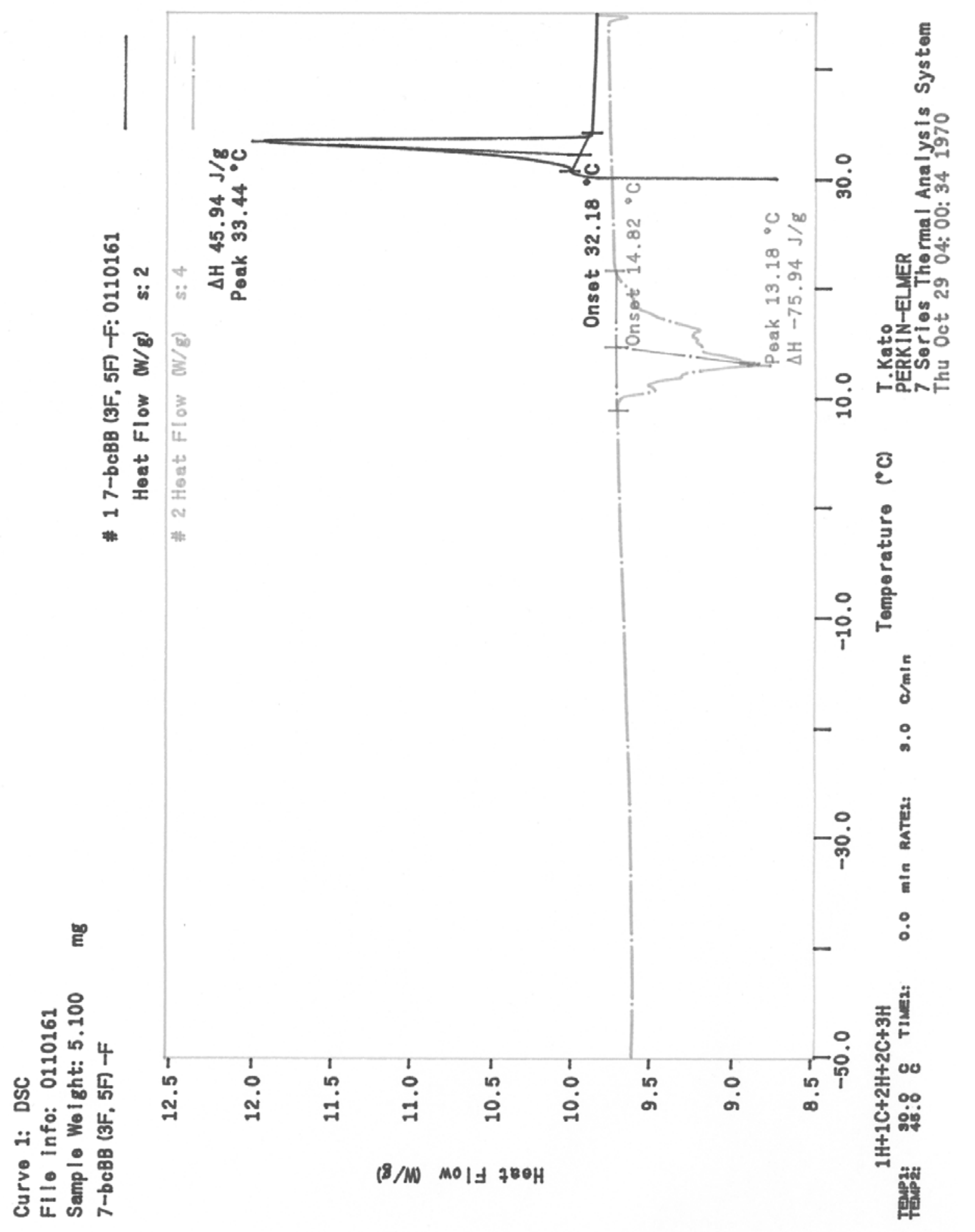




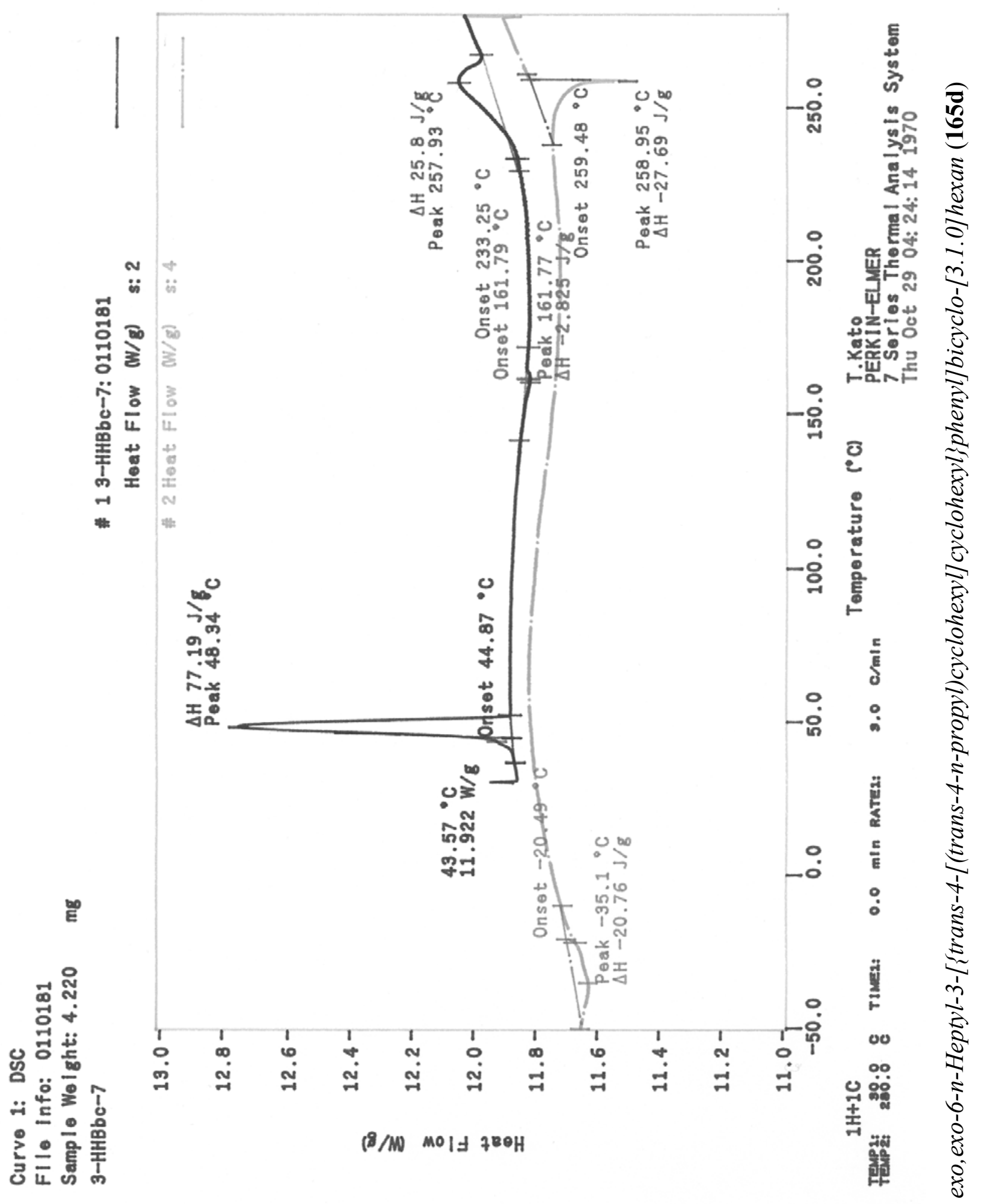




\section{Röntgen-Strukturanalysen}

4.1. exo,exo-Bicyclo[3.1.0]hexan-3,6-dicarbonsäure-3,6-di(4-n-pentylphenyl)ester (35a)

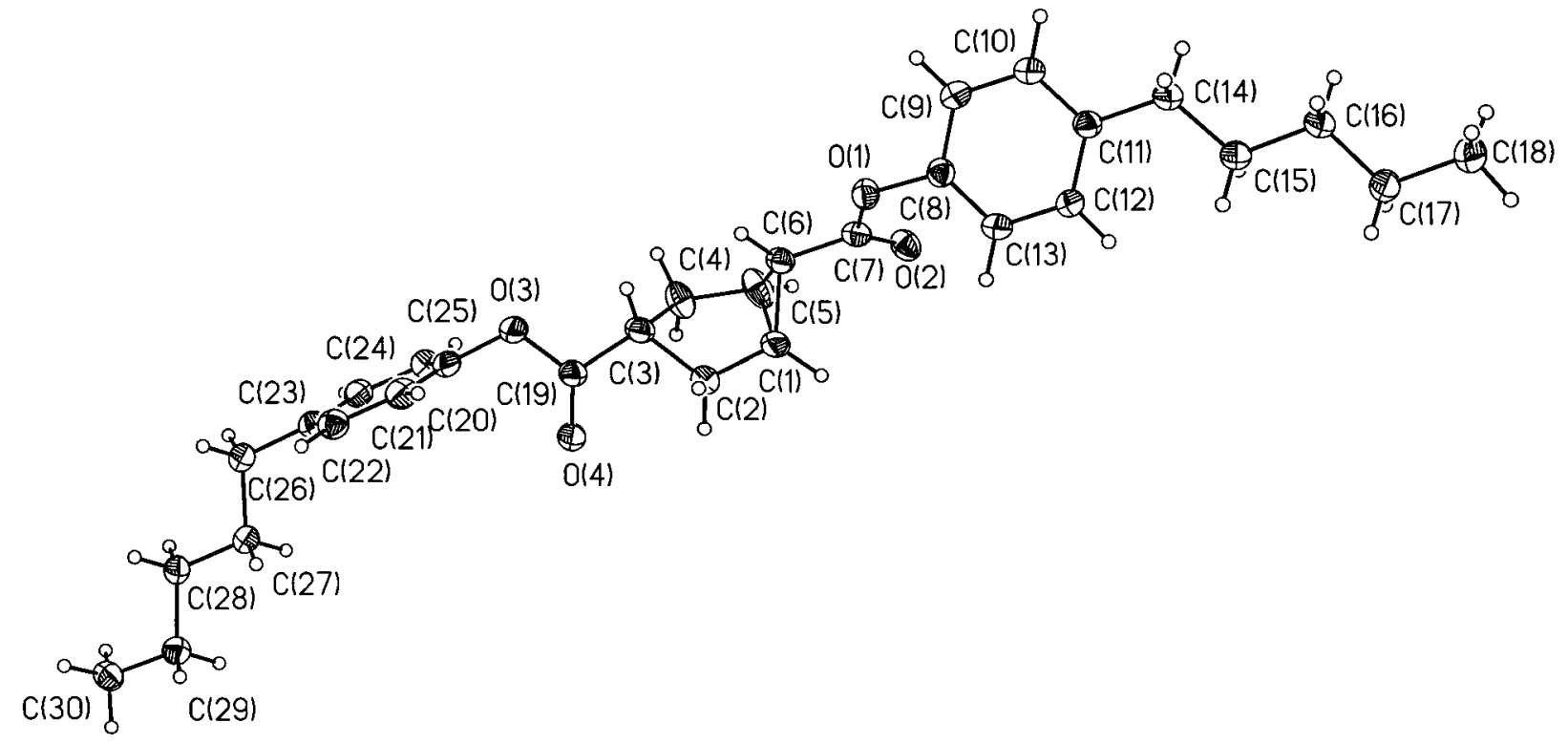

Die Nummerierung der Atome in der Röntgenstruktur stimmt nicht mit der IUPACNomenklatur überein.

Table 1. Crystal data and structure refinement for exo,exo-Bicyclo[3.1.0]hexan-3,6dicarbonsäure-3,6-di(4-n-pentylphenyl)ester (35a) .

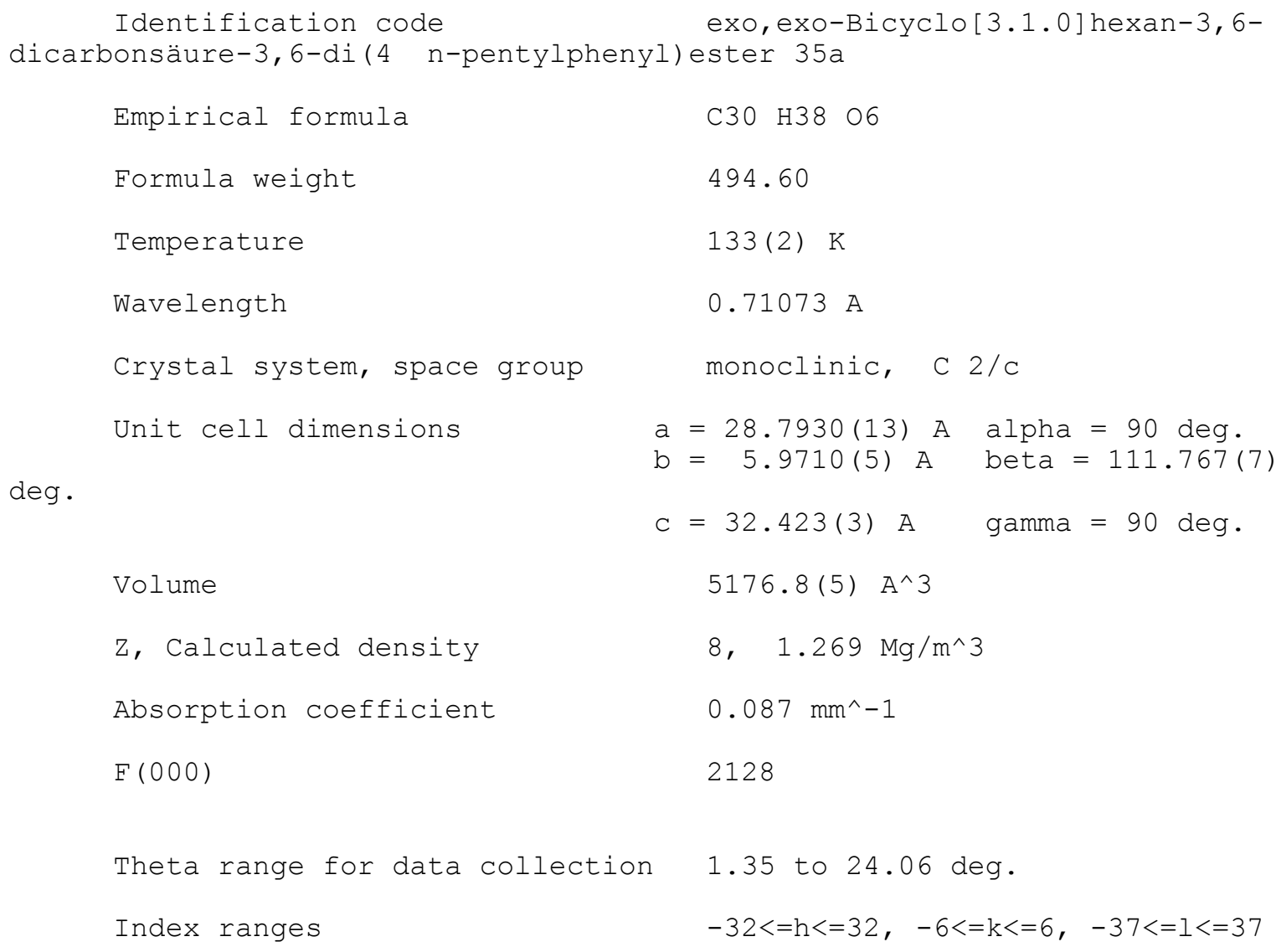




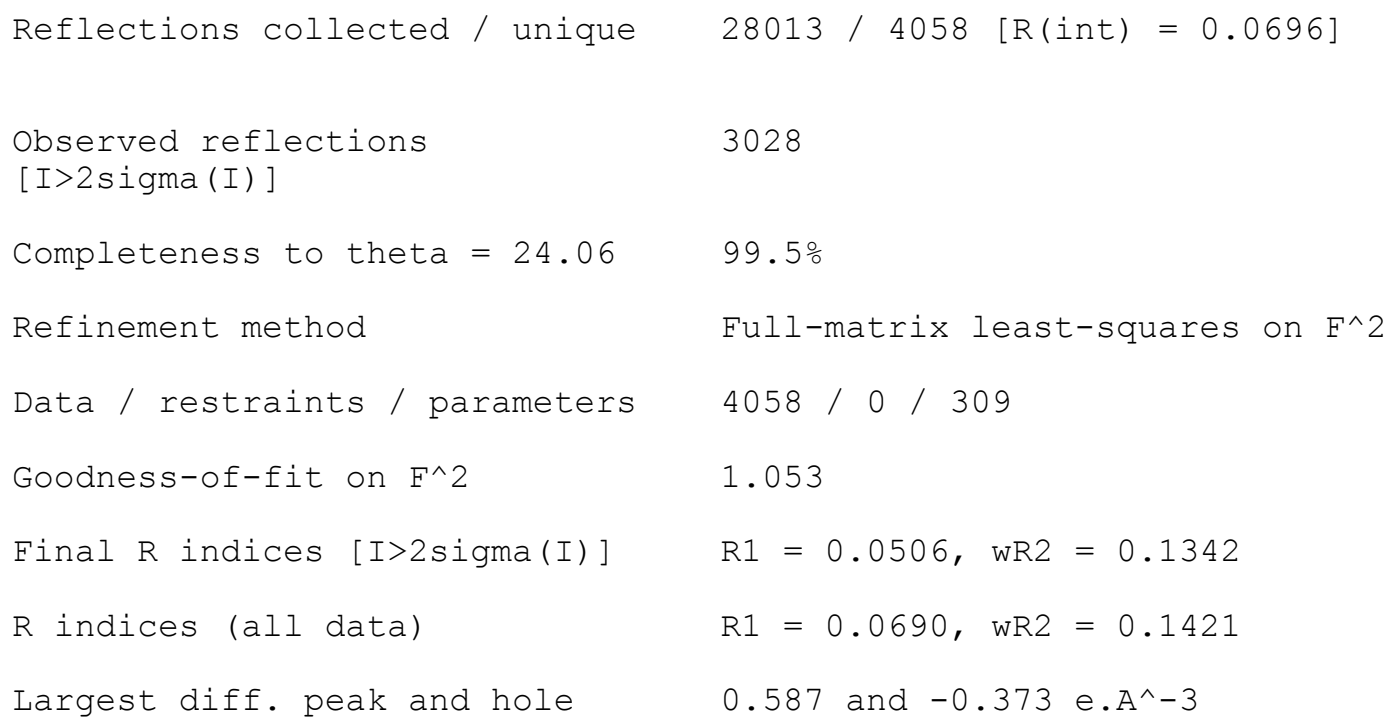


Table 2. Atomic coordinates and equivalent isotropic displacement parameters $\left(A^{\wedge} 2\right)$ for test.

$\mathrm{U}(\mathrm{eq})$ is defined as one third of the trace of the orthogonalized Uij tensor.

\begin{tabular}{|c|c|c|c|c|}
\hline & $\mathrm{x}$ & $\mathrm{y}$ & $\mathrm{z}$ & $\mathrm{U}(\mathrm{eq})$ \\
\hline$O(1)$ & $0.0983(1)$ & $0.4241(3)$ & $0.0868(1)$ & $0.0459(4)$ \\
\hline$O(2)$ & $0.0241(1)$ & $0.3003(3)$ & $0.0381(1)$ & $0.0516(4)$ \\
\hline$O(3)$ & $0.2093(1)$ & $-0.1815(3)$ & $-0.0413(1)$ & $0.0610(5)$ \\
\hline$O(4)$ & $0.1575(1)$ & $0.0143(4)$ & $-0.0979(1)$ & $0.0804(7)$ \\
\hline$C(1)$ & $0.0869(1)$ & $0.2953(4)$ & $-0.0195(1)$ & $0.0585(7)$ \\
\hline$C(2)$ & $0.1228(1)$ & $0.2742(6)$ & $-0.0403(1)$ & $0.0707(9)$ \\
\hline$C(3)$ & $0.1514(1)$ & $0.0495(4)$ & $-0.0264(1)$ & $0.0458(6)$ \\
\hline$C(4)$ & $0.1085(1)$ & $-0.0916(5)$ & $-0.0237(1)$ & $0.0585(7)$ \\
\hline$C(5)$ & $0.0766(1)$ & $0.0410(5)$ & $-0.0073(1)$ & $0.0668(8)$ \\
\hline$C(6)$ & $0.1003(1)$ & $0.2116(4)$ & $0.0276(1)$ & $0.0431(5)$ \\
\hline$C(7)$ & $0.0687(1)$ & $0.3089(4)$ & $0.0500(1)$ & $0.0420(5)$ \\
\hline$C(8)$ & $0.0756(1)$ & $0.5628(4)$ & $0.1090(1)$ & $0.0417(5)$ \\
\hline$C(9)$ & $0.0871(1)$ & $0.5240(4)$ & $0.1537(1)$ & $0.0503(6)$ \\
\hline$C(10)$ & $0.0692(1)$ & $0.6696(4)$ & $0.1774(1)$ & $0.0502(6)$ \\
\hline $\mathrm{C}(11)$ & $0.0401(1)$ & $0.8535(4)$ & $0.1577(1)$ & $0.0424(5)$ \\
\hline C (12) & $0.0293(1)$ & $0.8882(4)$ & $0.1126(1)$ & $0.0497(6)$ \\
\hline$C(13)$ & $0.0471(1)$ & $0.7433(4)$ & $0.0883(1)$ & $0.0510(6)$ \\
\hline$C(14)$ & $0.0220(1)$ & $1.0129(4)$ & $0.1849(1)$ & $0.0465(6)$ \\
\hline$C(15)$ & $-0.0208(1)$ & $1.1657(4)$ & $0.1590(1)$ & 0.0475 (6) \\
\hline$C(16)$ & $-0.0372(1)$ & $1.3195(4)$ & $0.1884(1)$ & $0.0479(6)$ \\
\hline$C(17)$ & $-0.0769(1)$ & $1.4855(5)$ & $0.1625(1)$ & $0.0615(7)$ \\
\hline$C(18)$ & $-0.0944(1)$ & $1.6388(5)$ & $0.1912(1)$ & $0.0638(7)$ \\
\hline C (19) & $0.1719(1)$ & $-0.0356(4)$ & $-0.0598(1)$ & $0.0461(6)$ \\
\hline$C(24)$ & $0.2447(1)$ & $-0.5622(4)$ & $-0.1139(1)$ & $0.0480(6)$ \\
\hline$C(20)$ & $0.2334(1)$ & $-0.2684(4)$ & $-0.0688(1)$ & $0.0480(6)$ \\
\hline$C(21)$ & $0.2726(1)$ & $-0.1531(4)$ & $-0.0728(1)$ & $0.0542(6)$ \\
\hline$C(22)$ & $0.2980(1)$ & $-0.2428(4)$ & $-0.0980(1)$ & $0.0518(6)$ \\
\hline C (23) & $0.2845(1)$ & $-0.4494(4)$ & $-0.1189(1)$ & $0.0454(6)$ \\
\hline$C(25)$ & $0.2191(1)$ & $-0.4726(4)$ & $-0.0889(1)$ & $0.0500(6)$ \\
\hline$C(26)$ & $0.3115(1)$ & $-0.5457(4)$ & $-0.1469(1)$ & $0.0510(6)$ \\
\hline C (27) & $0.2935(1)$ & $-0.4514(4)$ & $-0.1942(1)$ & $0.0463(6)$ \\
\hline$C(28)$ & $0.3187(1)$ & $-0.5644(4)$ & $-0.2224(1)$ & $0.0449(5)$ \\
\hline$C(29)$ & $0.3038(1)$ & $-0.4732(4)$ & $-0.2693(1)$ & $0.0486(6)$ \\
\hline$C(30)$ & $0.3271(1)$ & $-0.6047(5)$ & $-0.2968(1)$ & $0.0542(6)$ \\
\hline
\end{tabular}


Table 3. Bond lengths [pm] and angles [deg] for test.

\begin{tabular}{|c|c|}
\hline $\mathrm{O}(1)-\mathrm{C}(7)$ & $136.8(3)$ \\
\hline $\mathrm{O}(1)-\mathrm{C}(8)$ & $140.8(3)$ \\
\hline $\mathrm{O}(2)-\mathrm{C}(7)$ & $119.8(3)$ \\
\hline $\mathrm{O}(3)-\mathrm{C}(19)$ & $134.2(3)$ \\
\hline$O(3)-C(20)$ & $141.5(3)$ \\
\hline $\mathrm{O}(4)-\mathrm{C}(19)$ & $118.5(3)$ \\
\hline$C(1)-C(2)$ & $143.6(3)$ \\
\hline$C(1)-C(6)$ & $151.4(3)$ \\
\hline$C(1)-C(5)$ & $162.3(4)$ \\
\hline$C(2)-C(3)$ & $155.1(4)$ \\
\hline$C(3)-C(19)$ & $150.3(3)$ \\
\hline$C(3)-C(4)$ & $152.3(3)$ \\
\hline$C(4)-C(5)$ & $145.5(4)$ \\
\hline$C(5)-C(6)$ & $148.6(3)$ \\
\hline$C(6)-C(7)$ & $147.5(3)$ \\
\hline$C(8)-C(13)$ & $137.0(3)$ \\
\hline$C(8)-C(9)$ & $138.0(3)$ \\
\hline$C(9)-C(10)$ & $138.1(3)$ \\
\hline$C(10)-C(11)$ & $138.5(3)$ \\
\hline$C(11)-C(12)$ & $139.1(3)$ \\
\hline$C(11)-C(14)$ & $151.6(3)$ \\
\hline$C(12)-C(13)$ & $138.9(3)$ \\
\hline$C(14)-C(15)$ & $151.2(3)$ \\
\hline$C(15)-C(16)$ & $152.2(3)$ \\
\hline$C(16)-C(17)$ & $151.0(3)$ \\
\hline$C(17)-C(18)$ & $151.9(3)$ \\
\hline$C(24)-C(25)$ & $138.9(3)$ \\
\hline$C(24)-C(23)$ & $139.0(3)$ \\
\hline$C(20)-C(21)$ & $136.8(4)$ \\
\hline$C(20)-C(25)$ & $137.3(4)$ \\
\hline$C(21)-C(22)$ & $139.0(3)$ \\
\hline$C(22)-C(23)$ & $139.2(3)$ \\
\hline$C(23)-C(26)$ & $150.9(3)$ \\
\hline$C(26)-C(27)$ & $153.4(3)$ \\
\hline$C(27)-C(28)$ & $151.9(3)$ \\
\hline$C(28)-C(29)$ & $152.0(3)$ \\
\hline$C(29)-C(30)$ & $151.9(3)$ \\
\hline$C(7)-O(1)-C(8)$ & $119.06(17)$ \\
\hline$C(19)-O(3)-C(20)$ & $117.18(16)$ \\
\hline$C(2)-C(1)-C(6)$ & $119.1(2)$ \\
\hline$C(2)-C(1)-C(5)$ & $105.1(2)$ \\
\hline$C(6)-C(1)-C(5)$ & $56.42(15)$ \\
\hline$C(1)-C(2)-C(3)$ & $109.6(2)$ \\
\hline$C(19)-C(3)-C(4)$ & $113.4(2)$ \\
\hline$C(19)-C(3)-C(2)$ & $112.96(19)$ \\
\hline$C(4)-C(3)-C(2)$ & $98.2(2)$ \\
\hline$C(5)-C(4)-C(3)$ & $110.7(2)$ \\
\hline$C(4)-C(5)-C(6)$ & $118.6(2)$ \\
\hline$C(4)-C(5)-C(1)$ & $102.9(2)$ \\
\hline$C(6)-C(5)-C(1)$ & $58.08(17)$ \\
\hline$C(7)-C(6)-C(5)$ & $116.8(2)$ \\
\hline$C(7)-C(6)-C(1)$ & $113.0(2)$ \\
\hline$C(5)-C(6)-C(1)$ & $65.50(18)$ \\
\hline $\mathrm{O}(2)-\mathrm{C}(7)-\mathrm{O}(1)$ & $123.2(2)$ \\
\hline $\mathrm{O}(2)-\mathrm{C}(7)-\mathrm{C}(6)$ & $127.5(2)$ \\
\hline$O(1)-C(7)-C(6)$ & $109.23(18)$ \\
\hline$C(13)-C(8)-C(9)$ & $120.8(2)$ \\
\hline$C(13)-C(8)-O(1)$ & $121.31(19)$ \\
\hline$C(9)-C(8)-O(1)$ & $117.6(2)$ \\
\hline$C(10)-C(9)-C(8)$ & $119.0(2)$ \\
\hline$C(9)-C(10)-C(11)$ & $121.9(2)$ \\
\hline
\end{tabular}




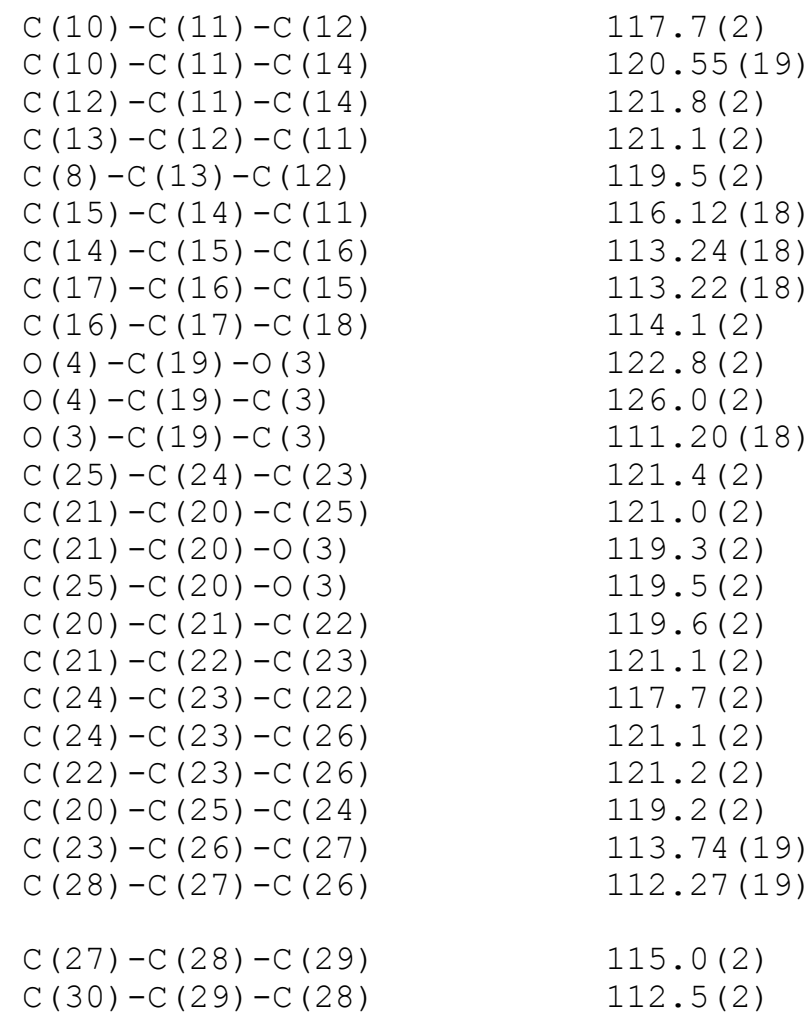

Symmetry transformations used to generate equivalent atoms: 
Table 4. Anisotropic displacement parameters $\left(A^{\wedge} 2\right)$ for test. The anisotropic displacement factor exponent takes the form: $-2 \mathrm{pi}^{\wedge} 2$ [ $\mathrm{h}^{\wedge} 2 \mathrm{a}^{\star \wedge} 2 \mathrm{U11}+\ldots+2 \mathrm{~h} \mathrm{k} \mathrm{a}^{\star} \mathrm{b} \mathrm{b}^{\star} \mathrm{U} 12$ ]

$\mathrm{U} 11$

$\mathrm{U} 22$

U33

U23

U13

$\mathrm{U} 12$

\begin{tabular}{|c|c|c|c|c|c|}
\hline $\begin{array}{l}0(1) \quad 0.0414(9) \\
0.0028(7)\end{array}$ & $0.0493(9)$ & $0.0456(8)$ & $-0.0066(7)$ & $0.0143(7)$ & \\
\hline $\begin{array}{ll}0(2) & 0.0434(10) \\
0.0080(8)\end{array}$ & $0.0650(11)$ & $0.0492(9)$ & $-0.0063(8)$ & $0.0206(7)$ & - \\
\hline $\begin{array}{l}0(3) \quad 0.0733(12) \\
0.0310(10)\end{array}$ & $0.0724(12)$ & $0.0385(8)$ & $0.0077(8)$ & $0.0220(8)$ & \\
\hline $\begin{array}{ll}0(4) & 0.0772(13) \\
0.0506(13)\end{array}$ & $0.1290(19)$ & $0.0386(10)$ & $0.0193(11)$ & $0.0256(9)$ & \\
\hline $\begin{array}{ll}C(1) & 0.087(2) \\
0.0241(14)\end{array}$ & $0.0479(14)$ & $0.0525(14)$ & $0.0159(11)$ & $0.0401(14)$ & \\
\hline $\begin{array}{ll}C(2) & 0.0745(19) \\
0.0366(17)\end{array}$ & $0.096(2)$ & $0.0512(14)$ & $0.0186(15)$ & $0.0349(14)$ & \\
\hline $\begin{array}{ll}C(3) & 0.0435(13) \\
0.0013(11)\end{array}$ & $0.0585(15)$ & $0.0349(11)$ & $0.0032(10)$ & $0.0141(10)$ & - \\
\hline $\begin{array}{l}C(4) \quad 0.0631(16) \\
0.0106(13)\end{array}$ & $0.0530(15)$ & $0.0721(17)$ & $-0.0175(13)$ & $0.0398(14)$ & - \\
\hline $\begin{array}{ll}C(5) & 0.0521(15) \\
0.0211(15)\end{array}$ & $0.093(2)$ & $0.0623(16)$ & $-0.0339(15)$ & $0.0289(13)$ & - \\
\hline $\begin{array}{ll}C(6) & 0.0401(12) \\
0.0025(11)\end{array}$ & $0.0521(14)$ & $0.0364(11)$ & $0.0018(10)$ & $0.0135(9)$ & - \\
\hline $\begin{array}{ll}C(7) & 0.0422(14) \\
0.0043(11)\end{array}$ & $0.0478(13)$ & $0.0352(11)$ & $0.0044(10)$ & $0.0134(10)$ & - \\
\hline $\begin{array}{ll}C(8) & 0.0367(12) \\
0.0008(10)\end{array}$ & $0.0455(13)$ & $0.0424(11)$ & $-0.0021(10)$ & $0.0143(10)$ & \\
\hline $\begin{array}{ll}C(9) & 0.0536(15) \\
0.0112(12)\end{array}$ & $0.0505(14)$ & $0.0445(13)$ & $0.0088(11)$ & $0.0155(11)$ & \\
\hline $\begin{array}{l}C(10) \quad 0.0572(15) \\
0.0094(13)\end{array}$ & $0.0558(15)$ & $0.0374(11)$ & $0.0066(11)$ & $0.0174(11)$ & \\
\hline $\begin{array}{l}C(11) \quad 0.0389(12) \\
0.0012(11)\end{array}$ & $0.0504(13)$ & $0.0366(11)$ & $0.0028(10)$ & $0.0126(9)$ & \\
\hline $\begin{array}{l}C(12) 0.0565(15) \\
0.0160(12)\end{array}$ & $0.0525(14)$ & $0.0393(12)$ & $0.0060(11)$ & $0.0167(11)$ & \\
\hline $\begin{array}{l}C(13) 0.0603(16) \\
0.0117(13)\end{array}$ & $0.0576(15)$ & $0.0363(11)$ & $0.0070(11)$ & $0.0194(11)$ & \\
\hline $\begin{array}{l}C(14) \quad 0.0455(13) \\
0.0065(11)\end{array}$ & $0.0569(15)$ & $0.0352(11)$ & $0.0021(10)$ & $0.0129(10)$ & \\
\hline $\begin{array}{l}C(15) \quad 0.0472(13) \\
0.0058(12)\end{array}$ & $0.0557(15)$ & $0.0360(11)$ & $-0.0005(10)$ & $0.0113(10)$ & \\
\hline $\begin{array}{l}C(16) \quad 0.0501(14) \\
0.0051(12)\end{array}$ & $0.0569(15)$ & $0.0355(11)$ & $-0.0009(10)$ & $0.0144(10)$ & \\
\hline $\begin{array}{l}C(17) \quad 0.0677(17) \\
0.0220(14)\end{array}$ & $0.0696(18)$ & $0.0410(13)$ & $-0.0040(12)$ & $0.0129(12)$ & \\
\hline $\begin{array}{l}C(18) \quad 0.0735(18) \\
0.0165(15)\end{array}$ & $0.0603(17)$ & $0.0565(15)$ & $-0.0042(13)$ & $0.0227(13)$ & \\
\hline $\begin{array}{l}C(19) \quad 0.0422(13) \\
0.0011(12)\end{array}$ & $0.0581(15)$ & $0.0369(12)$ & $0.0034(11)$ & $0.0135(10)$ & \\
\hline $\begin{array}{l}C(24) \quad 0.0499(14) \\
0.0059(12)\end{array}$ & $0.0469(13)$ & $0.0438(12)$ & $-0.0002(10)$ & $0.0136(11)$ & \\
\hline $\begin{array}{ll}C(20) & 0.0518(14) \\
0.0157(12)\end{array}$ & $0.0547(15)$ & $0.0350(11)$ & $0.0043(10)$ & $0.0130(10)$ & \\
\hline $\begin{array}{l}C(21) \quad 0.0618(16) \\
0.0050(13)\end{array}$ & $0.0518(15)$ & $0.0423(12)$ & $-0.0081(11)$ & $0.0115(11)$ & \\
\hline $\begin{array}{l}C(22) \quad 0.0479(14) \\
0.0012(12)\end{array}$ & $0.0554(15)$ & $0.0481(13)$ & $0.0006(11)$ & $0.0132(11)$ & - \\
\hline $\begin{array}{l}C(23) \quad 0.0428(13) \\
0.0079(11)\end{array}$ & $0.0516(14)$ & $0.0378(11)$ & $0.0029(10)$ & $0.0103(10)$ & \\
\hline
\end{tabular}




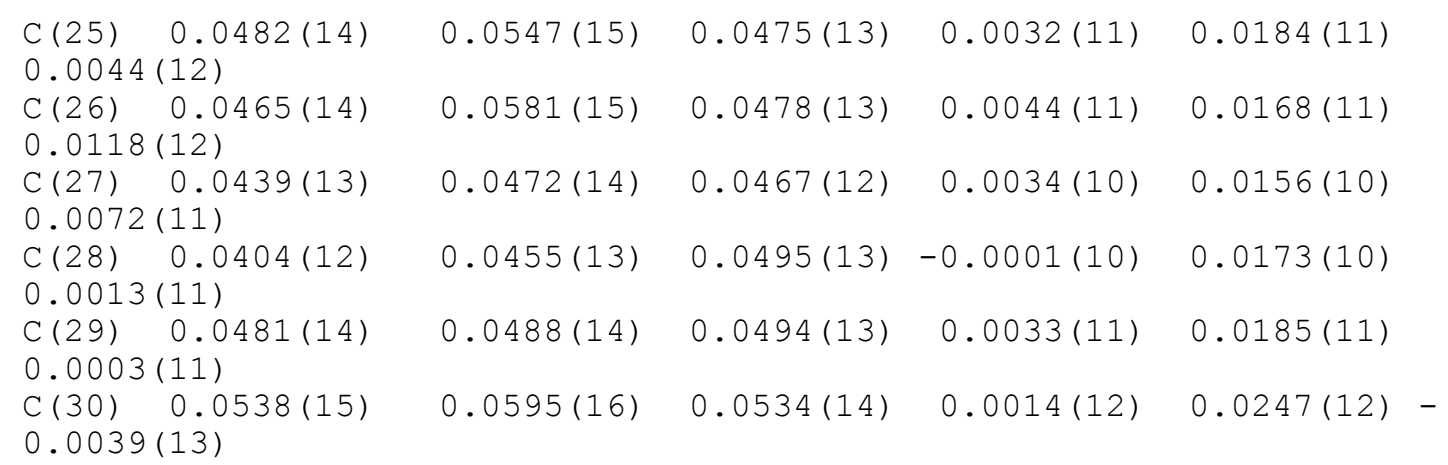


Table 5. Hydrogen coordinates and isotropic displacement parameters $\left(A^{\wedge} 2\right)$ for test.

\begin{tabular}{|c|c|c|c|c|}
\hline & $x$ & Y & z & $\mathrm{U}(\mathrm{eq})$ \\
\hline $\mathrm{H}(1 \mathrm{~A})$ & 0.0580 & 0.4002 & -0.0324 & 0.070 \\
\hline $\mathrm{H}(2 \mathrm{~A})$ & 0.1467 & 0.4006 & -0.0313 & 0.085 \\
\hline $\mathrm{H}(2 \mathrm{~B})$ & 0.1055 & 0.2788 & -0.0730 & 0.085 \\
\hline $\mathrm{H}(3 \mathrm{~A})$ & 0.1786 & 0.0635 & 0.0035 & 0.055 \\
\hline $\mathrm{H}(4 \mathrm{~A})$ & 0.0885 & -0.1510 & -0.0535 & 0.070 \\
\hline $\mathrm{H}(4 \mathrm{~B})$ & 0.1222 & -0.2204 & -0.0036 & 0.070 \\
\hline $\mathrm{H}(5 \mathrm{~A})$ & 0.0416 & -0.0092 & -0.0131 & 0.080 \\
\hline $\mathrm{H}(6 \mathrm{~A})$ & 0.1366 & 0.1922 & 0.0459 & 0.052 \\
\hline $\mathrm{H}(9 \mathrm{~A})$ & 0.1070 & 0.3991 & 0.1679 & 0.060 \\
\hline $\mathrm{H}(10 \mathrm{~A})$ & 0.0770 & 0.6428 & 0.2081 & 0.060 \\
\hline $\mathrm{H}(12 \mathrm{~A})$ & 0.0095 & 1.0132 & 0.0983 & 0.060 \\
\hline $\mathrm{H}(13 \mathrm{~A})$ & 0.0395 & 0.7690 & 0.0576 & 0.061 \\
\hline $\mathrm{H}(14 \mathrm{~A})$ & 0.0115 & 0.9234 & 0.2056 & 0.056 \\
\hline $\mathrm{H}(14 \mathrm{~B})$ & 0.0506 & 1.1071 & 0.2030 & 0.056 \\
\hline $\mathrm{H}(15 \mathrm{~A})$ & -0.0496 & 1.0731 & 0.1407 & 0.057 \\
\hline $\mathrm{H}(15 \mathrm{~B})$ & -0.0104 & 1.2582 & 0.1385 & 0.057 \\
\hline $\mathrm{H}(16 \mathrm{~A})$ & -0.0503 & 1.2272 & 0.2071 & 0.058 \\
\hline $\mathrm{H}(16 \mathrm{~B})$ & -0.0078 & 1.4026 & 0.2085 & 0.058 \\
\hline $\mathrm{H}(17 \mathrm{~A})$ & -0.1061 & 1.4020 & 0.1421 & 0.074 \\
\hline $\mathrm{H}(17 \mathrm{~B})$ & -0.0636 & 1.5785 & 0.1441 & 0.074 \\
\hline $\mathrm{H}(18 \mathrm{~A})$ & -0.1185 & 1.7471 & 0.1724 & 0.096 \\
\hline $\mathrm{H}(18 \mathrm{~B})$ & -0.0656 & 1.7188 & 0.2122 & 0.096 \\
\hline $\mathrm{H}(18 \mathrm{C})$ & -0.1103 & 1.5494 & 0.2077 & 0.096 \\
\hline $\mathrm{H}(24 \mathrm{~A})$ & 0.2348 & -0.7036 & -0.1278 & 0.058 \\
\hline $\mathrm{H}(21 \mathrm{~A})$ & 0.2824 & -0.0126 & -0.0585 & 0.065 \\
\hline $\mathrm{H}(22 \mathrm{~A})$ & 0.3249 & -0.1618 & -0.1010 & 0.062 \\
\hline $\mathrm{H}(25 \mathrm{~A})$ & 0.1919 & -0.5516 & -0.0858 & 0.060 \\
\hline $\mathrm{H}(26 \mathrm{~A})$ & 0.3069 & -0.7103 & -0.1485 & 0.061 \\
\hline $\mathrm{H}(26 \mathrm{~B})$ & 0.3477 & -0.5151 & -0.1320 & 0.061 \\
\hline $\mathrm{H}(27 \mathrm{~A})$ & 0.2568 & -0.4721 & -0.2085 & 0.056 \\
\hline $\mathrm{H}(27 \mathrm{~B})$ & 0.3004 & -0.2887 & -0.1928 & 0.056 \\
\hline $\mathrm{H}(28 \mathrm{~A})$ & 0.3107 & -0.7263 & -0.2242 & 0.054 \\
\hline $\mathrm{H}(28 \mathrm{~B})$ & 0.3553 & -0.5489 & -0.2071 & 0.054 \\
\hline $\mathrm{H}(29 \mathrm{~A})$ & 0.2670 & -0.4779 & -0.2840 & 0.058 \\
\hline $\mathrm{H}(29 \mathrm{~B})$ & 0.3144 & -0.3147 & -0.2678 & 0.058 \\
\hline $\mathrm{H}(30 \mathrm{~A})$ & 0.3156 & -0.5430 & -0.3270 & 0.081 \\
\hline $\mathrm{H}(30 \mathrm{~B})$ & 0.3170 & -0.7622 & -0.2981 & 0.081 \\
\hline $\mathrm{H}(30 \mathrm{C})$ & 0.3636 & -0.5938 & -0.2832 & 0.081 \\
\hline
\end{tabular}


4.2. 3-\{[4-(trans-4-n-Pentyl)cyclohexyl]-phenyl\} bicyclo[3.1.0]hexan-6-carbonsäure-tertbutylester 162a

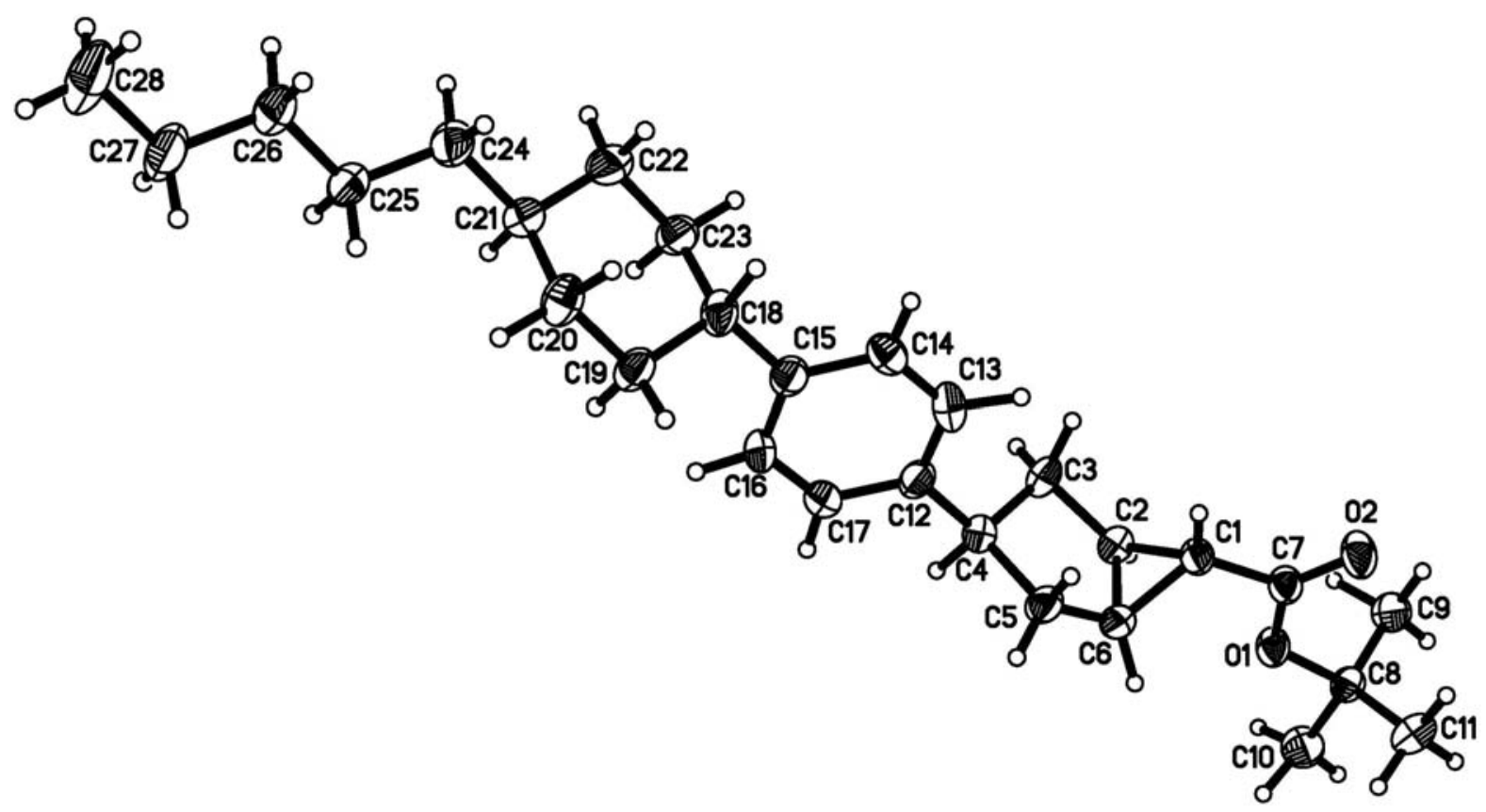

Table 1. Crystal data and structure refinement for 3-\{[4-(trans-4-n-Pentyl)cyclohexyl]phenyl \} bicyclo[3.1.0]hexan-6-carbonsäure-tertbutylester 162a

Identification code

3-\{[4-(trans-4-n-Pentyl)cyclohexyl]-

phenyl\} bicyclo[3.1.0]hexan-6-carbonsäure-tertbutylester 162a

Empirical formula

Formula weight

Temperature

Wavelength

Crystal system

Space group

Unit cell dimensions

Volume

$\mathrm{Z}$

Density (calculated)

Absorption coefficient

$\mathrm{F}(000)$

Crystal size

Theta range for data collection

Index ranges
$\mathrm{C}_{28} \mathrm{H}_{42} \mathrm{O}_{2}$

410.62

$120.0(2) \mathrm{K}$

$0.71073 \AA$

Monoclinic

$\mathrm{P} 21 / \mathrm{c}$

$$
\begin{array}{ll}
\mathrm{a}=20.0752(12) \AA & \alpha=90^{\circ} . \\
\mathrm{b}=9.9072(6) \AA & \beta=101.097(2)^{\circ} . \\
\mathrm{c}=12.7752(7) \AA & \gamma=90^{\circ} .
\end{array}
$$

2493.3(3) $\AA^{3}$

4

$1.094 \mathrm{Mg} / \mathrm{m}^{3}$

$0.066 \mathrm{~mm}^{-1}$

904

$0.28 \times 0.20 \times 0.03 \mathrm{~mm}^{3}$

2.07 to $30.64^{\circ}$.

$-27<=\mathrm{h}<=28,-14<=\mathrm{k}<=13,-17<=1<=18$ 
Reflections collected

Independent reflections

Completeness to theta $=30.64^{\circ}$

Max. and min. transmission

Refinement method

Data / restraints / parameters

Goodness-of-fit on $\mathrm{F}^{2}$

Final $\mathrm{R}$ indices $[\mathrm{I}>2 \operatorname{sigma}(\mathrm{I})]$

$\mathrm{R}$ indices (all data)

Largest diff. peak and hole
26230

$6150[\mathrm{R}(\mathrm{int})=0.1550]$

$79.8 \%$

0.9980 and 0.9817

Full-matrix least-squares on $\mathrm{F}^{2}$

$6150 / 0 / 439$

0.928

$\mathrm{R} 1=0.0841, \mathrm{wR} 2=0.1832$

$\mathrm{R} 1=0.1934, \mathrm{wR} 2=0.2190$

0.395 and -0.278 e. $\AA^{-3}$ 
Table 2. Atomic coordinates $\left(\mathrm{x} 10^{4}\right)$ and equivalent isotropic displacement parameters $\left(\AA^{2} \times 10^{3}\right)$ for 3-\{[4-(trans-4-n-Pentyl)cyclohexyl]-phenyl\}bicyclo[3.1.0]hexan-6-carbonsäure-tertbutylester 162a. U(eq) is defined as one third of the trace of the orthogonalized $U^{i j}$ tensor.

\begin{tabular}{|c|c|c|c|c|}
\hline Atom & $\mathrm{x}$ & $\mathrm{y}$ & $\mathrm{z}$ & $\mathrm{U}(\mathrm{eq})$ \\
\hline$\overline{\mathrm{O}(1)}$ & $4476(1)$ & $7403(2)$ & $11764(2)$ & $31(1)$ \\
\hline $\mathrm{O}(2)$ & $4236(1)$ & $9385(2)$ & $10902(2)$ & $43(1)$ \\
\hline $\mathrm{C}(1)$ & $5213(1)$ & $8156(3)$ & $10669(2)$ & $29(1)$ \\
\hline$C(2)$ & $5838(1)$ & $7568(3)$ & $11376(2)$ & $30(1)$ \\
\hline $\mathrm{C}(3)$ & $6516(2)$ & $7926(3)$ & $11058(3)$ & $33(1)$ \\
\hline$C(4)$ & $6607(2)$ & $6838(3)$ & $10243(3)$ & $36(1)$ \\
\hline $\mathrm{C}(5)$ & $5871(2)$ & $6538(4)$ & $9648(3)$ & $36(1)$ \\
\hline$C(6)$ & $5441(2)$ & $6730(3)$ & $10495(2)$ & $32(1)$ \\
\hline$C(7)$ & $4591(1)$ & $8399(3)$ & $11115(2)$ & $32(1)$ \\
\hline $\mathrm{C}(8)$ & $3885(1)$ & $7428(3)$ & $12294(2)$ & $29(1)$ \\
\hline$C(9)$ & $3936(2)$ & $8630(4)$ & $13047(3)$ & $39(1)$ \\
\hline $\mathrm{C}(10)$ & $3958(2)$ & $6113(4)$ & $12910(3)$ & $43(1)$ \\
\hline $\mathrm{C}(11)$ & $3234(2)$ & $7435(4)$ & $11463(3)$ & $43(1)$ \\
\hline$C(12)$ & $7099(1)$ & $7169(3)$ & $9523(2)$ & $32(1)$ \\
\hline $\mathrm{C}(13)$ & $7095(2)$ & $8388(3)$ & $8991(3)$ & $38(1)$ \\
\hline$C(14)$ & $7560(2)$ & $8668(3)$ & $8342(3)$ & $35(1)$ \\
\hline$C(15)$ & $8044(1)$ & $7705(3)$ & $8179(2)$ & 31(1) \\
\hline$C(16)$ & $8041(2)$ & $6482(3)$ & $8701(3)$ & $33(1)$ \\
\hline $\mathrm{C}(17)$ & $7584(2)$ & $6233(3)$ & $9359(3)$ & $34(1)$ \\
\hline $\mathrm{C}(18)$ & $8560(2)$ & $8065(3)$ & $7496(3)$ & $34(1)$ \\
\hline$C(19)$ & $8731(2)$ & $6916(3)$ & $6797(3)$ & $37(1)$ \\
\hline $\mathrm{C}(20)$ & $9253(2)$ & $7333(4)$ & $6135(3)$ & $39(1)$ \\
\hline $\mathrm{C}(21)$ & $9907(2)$ & $7912(3)$ & $6812(3)$ & $34(1)$ \\
\hline $\mathrm{C}(22)$ & $9727(2)$ & $9045(4)$ & $7518(3)$ & $43(1)$ \\
\hline $\mathrm{C}(23)$ & $9215(2)$ & $8603(3)$ & $8190(3)$ & $37(1)$ \\
\hline $\mathrm{C}(24)$ & $10415(2)$ & $8388(3)$ & $6131(3)$ & 41(1) \\
\hline $\mathrm{C}(25)$ & $10759(2)$ & $7264(3)$ & $5632(3)$ & $35(1)$ \\
\hline$C(26)$ & $11238(2)$ & $7738(4)$ & $4917(3)$ & 41(1) \\
\hline $\mathrm{C}(27)$ & $11586(2)$ & $6623(4)$ & $4443(4)$ & $52(1)$ \\
\hline $\mathrm{C}(28)$ & $12030(2)$ & $7087(6)$ & $3674(4)$ & $70(2)$ \\
\hline
\end{tabular}


Table 3. Bond lengths $[\AA]$ and angles $\left[^{\circ}\right]$ for 3-\{[4-(trans-4-n-Pentyl)cyclohexyl $]$ phenyl bicyclo[3.1.0] hexan-6-carbonsäure-tertbutylester 162a.

\begin{tabular}{|l|l|l|l|l|l|}
\hline $\mathrm{O}(1)-\mathrm{C}(7)$ & $1.338(3)$ & $\mathrm{C}(5)-\mathrm{C}(6)$ & $1.521(4)$ & $\mathrm{C}(18)-\mathrm{C}(19)$ & $1.526(4)$ \\
\hline $\mathrm{O}(1)-\mathrm{C}(8)$ & $1.475(3)$ & $\mathrm{C}(8)-\mathrm{C}(10)$ & $1.514(4)$ & $\mathrm{C}(18)-\mathrm{C}(23)$ & $1.533(4)$ \\
\hline $\mathrm{O}(2)-\mathrm{C}(7)$ & $1.208(3)$ & $\mathrm{C}(8)-\mathrm{C}(11)$ & $1.516(4)$ & $\mathrm{C}(19)-\mathrm{C}(20)$ & $1.525(4)$ \\
\hline $\mathrm{C}(1)-\mathrm{C}(7)$ & $1.489(4)$ & $\mathrm{C}(8)-\mathrm{C}(9)$ & $1.523(4)$ & $\mathrm{C}(20)-\mathrm{C}(21)$ & $1.537(4)$ \\
\hline $\mathrm{C}(1)-\mathrm{C}(2)$ & $1.513(4)$ & $\mathrm{C}(12)-\mathrm{C}(13)$ & $1.385(4)$ & $\mathrm{C}(21)-\mathrm{C}(22)$ & $1.526(5)$ \\
\hline $\mathrm{C}(1)-\mathrm{C}(6)$ & $1.515(4)$ & $\mathrm{C}(12)-\mathrm{C}(17)$ & $1.389(4)$ & $\mathrm{C}(21)-\mathrm{C}(24)$ & $1.537(4)$ \\
\hline $\mathrm{C}(2)-\mathrm{C}(6)$ & $1.498(4)$ & $\mathrm{C}(13)-\mathrm{C}(14)$ & $1.391(5)$ & $\mathrm{C}(22)-\mathrm{C}(23)$ & $1.523(4)$ \\
\hline $\mathrm{C}(2)-\mathrm{C}(3)$ & $1.536(4)$ & $\mathrm{C}(14)-\mathrm{C}(15)$ & $1.406(4)$ & $\mathrm{C}(24)-\mathrm{C}(25)$ & $1.514(4)$ \\
\hline $\mathrm{C}(3)-\mathrm{C}(4)$ & $1.534(4)$ & $\mathrm{C}(15)-\mathrm{C}(16)$ & $1.384(4)$ & $\mathrm{C}(25)-\mathrm{C}(26)$ & $1.523(4)$ \\
\hline $\mathrm{C}(4)-\mathrm{C}(12)$ & $1.508(4)$ & $\mathrm{C}(15)-\mathrm{C}(18)$ & $1.519(4)$ & $\mathrm{C}(26)-\mathrm{C}(27)$ & $1.496(5)$ \\
\hline $\mathrm{C}(4)-\mathrm{C}(5)$ & $1.556(4)$ & $\mathrm{C}(16)-\mathrm{C}(17)$ & $1.381(4)$ & $\mathrm{C}(27)-\mathrm{C}(28)$ & $1.519(6)$ \\
\hline
\end{tabular}

\begin{tabular}{|l|l|l|l|}
\hline $\mathrm{C}(7)-\mathrm{O}(1)-\mathrm{C}(8)$ & $121.5(2)$ & $\mathrm{C}(13)-\mathrm{C}(12)-\mathrm{C}(17)$ & $116.5(3)$ \\
\hline $\mathrm{C}(7)-\mathrm{C}(1)-\mathrm{C}(2)$ & $119.6(2)$ & $\mathrm{C}(13)-\mathrm{C}(12)-\mathrm{C}(4)$ & $123.1(3)$ \\
\hline $\mathrm{C}(7)-\mathrm{C}(1)-\mathrm{C}(6)$ & $120.4(2)$ & $\mathrm{C}(17)-\mathrm{C}(12)-\mathrm{C}(4)$ & $120.4(3)$ \\
\hline $\mathrm{C}(2)-\mathrm{C}(1)-\mathrm{C}(6)$ & $59.3(2)$ & $\mathrm{C}(12)-\mathrm{C}(13)-\mathrm{C}(14)$ & $121.7(3)$ \\
\hline $\mathrm{C}(6)-\mathrm{C}(2)-\mathrm{C}(1)$ & $60.39(19)$ & $\mathrm{C}(13)-\mathrm{C}(14)-\mathrm{C}(15)$ & $121.1(3)$ \\
\hline $\mathrm{C}(6)-\mathrm{C}(2)-\mathrm{C}(3)$ & $107.4(2)$ & $\mathrm{C}(16)-\mathrm{C}(15)-\mathrm{C}(14)$ & $117.0(3)$ \\
\hline $\mathrm{C}(1)-\mathrm{C}(2)-\mathrm{C}(3)$ & $115.4(3)$ & $\mathrm{C}(16)-\mathrm{C}(15)-\mathrm{C}(18)$ & $123.4(3)$ \\
\hline $\mathrm{C}(4)-\mathrm{C}(3)-\mathrm{C}(2)$ & $104.2(2)$ & $\mathrm{C}(14)-\mathrm{C}(15)-\mathrm{C}(18)$ & $119.5(3)$ \\
\hline $\mathrm{C}(12)-\mathrm{C}(4)-\mathrm{C}(3)$ & $116.0(3)$ & $\mathrm{C}(17)-\mathrm{C}(16)-\mathrm{C}(15)$ & $121.1(3)$ \\
\hline $\mathrm{C}(12)-\mathrm{C}(4)-\mathrm{C}(5)$ & $114.5(3)$ & $\mathrm{C}(16)-\mathrm{C}(17)-\mathrm{C}(12)$ & $122.6(3)$ \\
\hline $\mathrm{C}(3)-\mathrm{C}(4)-\mathrm{C}(5)$ & $103.8(2)$ & $\mathrm{C}(15)-\mathrm{C}(18)-\mathrm{C}(19)$ & $114.6(3)$ \\
\hline $\mathrm{C}(6)-\mathrm{C}(5)-\mathrm{C}(4)$ & $104.2(3)$ & $\mathrm{C}(15)-\mathrm{C}(18)-\mathrm{C}(23)$ & $110.7(2)$ \\
\hline $\mathrm{C}(2)-\mathrm{C}(6)-\mathrm{C}(1)$ & $60.29(19)$ & $\mathrm{C}(19)-\mathrm{C}(18)-\mathrm{C}(23)$ & $109.4(2)$ \\
\hline $\mathrm{C}(2)-\mathrm{C}(6)-\mathrm{C}(5)$ & $108.1(2)$ & $\mathrm{C}(20)-\mathrm{C}(19)-\mathrm{C}(18)$ & $112.3(3)$ \\
\hline $\mathrm{C}(1)-\mathrm{C}(6)-\mathrm{C}(5)$ & $116.7(3)$ & $\mathrm{C}(19)-\mathrm{C}(20)-\mathrm{C}(21)$ & $113.1(3)$ \\
\hline $\mathrm{O}(2)-\mathrm{C}(7)-\mathrm{O}(1)$ & $124.8(3)$ & $\mathrm{C}(22)-\mathrm{C}(21)-\mathrm{C}(24)$ & $111.6(3)$ \\
\hline $\mathrm{O}(2)-\mathrm{C}(7)-\mathrm{C}(1)$ & $123.1(3)$ & $\mathrm{C}(22)-\mathrm{C}(21)-\mathrm{C}(20)$ & $109.5(3)$ \\
\hline $\mathrm{O}(1)-\mathrm{C}(7)-\mathrm{C}(1)$ & $112.0(2)$ & $\mathrm{C}(24)-\mathrm{C}(21)-\mathrm{C}(20)$ & $112.5(3)$ \\
\hline $\mathrm{O}(1)-\mathrm{C}(8)-\mathrm{C}(10)$ & $102.5(2)$ & $\mathrm{C}(23)-\mathrm{C}(22)-\mathrm{C}(21)$ & $112.5(3)$ \\
\hline $\mathrm{O}(1)-\mathrm{C}(8)-\mathrm{C}(11)$ & $109.8(2)$ & $\mathrm{C}(22)-\mathrm{C}(23)-\mathrm{C}(18)$ & $111.8(3)$ \\
\hline $\mathrm{C}(10)-\mathrm{C}(8)-\mathrm{C}(11)$ & $110.6(3)$ & $\mathrm{C}(25)-\mathrm{C}(24)-\mathrm{C}(21)$ & $114.8(3)$ \\
\hline $\mathrm{O}(1)-\mathrm{C}(8)-\mathrm{C}(9)$ & $109.9(2)$ & $\mathrm{C}(24)-\mathrm{C}(25)-\mathrm{C}(26)$ & $114.7(3)$ \\
\hline $\mathrm{C}(10)-\mathrm{C}(8)-\mathrm{C}(9)$ & $110.9(3)$ & $\mathrm{C}(27)-\mathrm{C}(26)-\mathrm{C}(25)$ & $114.4(3)$ \\
\hline $\mathrm{C}(11)-\mathrm{C}(8)-\mathrm{C}(9)$ & $112.6(3)$ & $\mathrm{C}(26)-\mathrm{C}(27)-\mathrm{C}(28)$ & $114.5(4)$ \\
\hline
\end{tabular}


Table 4. Anisotropic displacement parameters $\left(\AA^{2} \times 10^{3}\right)$ for 3-\{[4-(trans-4-n-Pentyl)cyclohexyl]phenyl \} bicyclo[3.1.0]hexan-6-carbonsäure-tertbutylester 162a. The anisotropic displacement factor exponent takes the form: $-2 \pi^{2}\left[\mathrm{~h}^{2} \mathrm{a}^{* 2} \mathrm{U}^{11}+\ldots+2 \mathrm{~h} \mathrm{k} \mathrm{a}^{*} \mathrm{~b}^{*} \mathrm{U}^{12}\right]$

\begin{tabular}{|c|c|c|c|c|c|c|}
\hline Atom & $\mathrm{U}^{11}$ & $\mathrm{U}^{22}$ & $\mathrm{U}^{33}$ & $\mathrm{U}^{23}$ & $\mathrm{U}^{13}$ & $\mathrm{U}^{12}$ \\
\hline$\overline{\mathrm{O}(1)}$ & $29(1)$ & $32(1)$ & $35(1)$ & $6(1)$ & $15(1)$ & $3(1)$ \\
\hline $\mathrm{O}(2)$ & $38(1)$ & $38(1)$ & $59(2)$ & $19(1)$ & $21(1)$ & $15(1)$ \\
\hline$C(1)$ & $28(2)$ & $27(2)$ & $34(2)$ & $6(1)$ & $11(1)$ & $3(1)$ \\
\hline$C(2)$ & $27(1)$ & $34(2)$ & $29(2)$ & $3(1)$ & $8(1)$ & $2(1)$ \\
\hline$C(3)$ & $22(2)$ & $46(2)$ & $29(2)$ & $-6(2)$ & $2(1)$ & $-1(1)$ \\
\hline$C(4)$ & $31(2)$ & $33(2)$ & $45(2)$ & $-3(2)$ & $12(2)$ & 1(1) \\
\hline $\mathrm{C}(5)$ & $28(2)$ & $39(2)$ & $45(2)$ & $-12(2)$ & $15(2)$ & $-6(1)$ \\
\hline $\mathrm{C}(6)$ & $27(2)$ & $27(2)$ & $41(2)$ & $-4(2)$ & $9(1)$ & $-3(1)$ \\
\hline$C(7)$ & $29(2)$ & $31(2)$ & $36(2)$ & $5(2)$ & $10(1)$ & $4(1)$ \\
\hline $\mathrm{C}(8)$ & $26(1)$ & $32(2)$ & $30(2)$ & 2(1) & $10(1)$ & $-2(1)$ \\
\hline$C(9)$ & $37(2)$ & $40(2)$ & $40(2)$ & $-3(2)$ & $13(2)$ & $-2(2)$ \\
\hline$C(10)$ & $46(2)$ & $38(2)$ & $49(2)$ & $6(2)$ & $18(2)$ & $-3(2)$ \\
\hline $\mathrm{C}(11)$ & $31(2)$ & $50(2)$ & $46(2)$ & $2(2)$ & $7(2)$ & $-7(2)$ \\
\hline$C(12)$ & $22(1)$ & $38(2)$ & $38(2)$ & $-7(2)$ & $6(1)$ & $-3(1)$ \\
\hline $\mathrm{C}(13)$ & $32(2)$ & $41(2)$ & $41(2)$ & $-5(2)$ & $8(2)$ & $13(1)$ \\
\hline$C(14)$ & $38(2)$ & $27(2)$ & $38(2)$ & $3(2)$ & $4(2)$ & $4(1)$ \\
\hline$C(15)$ & $25(1)$ & $37(2)$ & $30(2)$ & $-4(1)$ & $5(1)$ & $-2(1)$ \\
\hline$C(16)$ & $29(2)$ & $32(2)$ & $42(2)$ & $-3(2)$ & $14(1)$ & $8(1)$ \\
\hline$C(17)$ & $32(2)$ & $25(2)$ & $46(2)$ & $0(2)$ & $10(2)$ & $1(1)$ \\
\hline $\mathrm{C}(18)$ & $31(2)$ & $38(2)$ & $34(2)$ & $2(2)$ & 11(1) & $5(1)$ \\
\hline$C(19)$ & $28(2)$ & $48(2)$ & $36(2)$ & $-11(2)$ & $11(2)$ & $-8(1)$ \\
\hline $\mathrm{C}(20)$ & $35(2)$ & $50(2)$ & $32(2)$ & $-5(2)$ & $10(1)$ & $2(2)$ \\
\hline $\mathrm{C}(21)$ & $31(2)$ & $32(2)$ & $41(2)$ & $1(2)$ & $10(1)$ & $-3(1)$ \\
\hline $\mathrm{C}(22)$ & $44(2)$ & $39(2)$ & $51(2)$ & $-5(2)$ & $21(2)$ & $-12(2)$ \\
\hline $\mathrm{C}(23)$ & $37(2)$ & $37(2)$ & $40(2)$ & $-14(2)$ & $14(2)$ & $-8(1)$ \\
\hline $\mathrm{C}(24)$ & $42(2)$ & $37(2)$ & $50(2)$ & $5(2)$ & $23(2)$ & $0(2)$ \\
\hline$C(25)$ & $27(2)$ & $41(2)$ & $39(2)$ & $-2(2)$ & $9(1)$ & $-1(1)$ \\
\hline $\mathrm{C}(26)$ & $35(2)$ & $49(2)$ & $42(2)$ & $1(2)$ & $16(2)$ & $2(2)$ \\
\hline $\mathrm{C}(27)$ & $32(2)$ & $68(3)$ & $59(3)$ & $-13(2)$ & $18(2)$ & $-6(2)$ \\
\hline $\mathrm{C}(28)$ & $42(2)$ & $112(4)$ & $60(3)$ & $-22(3)$ & $25(2)$ & $-7(3)$ \\
\hline
\end{tabular}


Table 5. Hydrogen coordinates ( x 10 $)$ and isotropic displacement parameters $\left(\AA^{2} \times 10^{3}\right)$

for 3-\{[4-(trans-4-n-Pentyl)cyclohexyl]-phenyl\} bicyclo[3.1.0]hexan-6-carbonsäure-tertbutylester 162a.

\begin{tabular}{|c|c|c|c|c|}
\hline Atom & $\mathrm{x}$ & $\mathrm{y}$ & $\mathrm{z}$ & $\mathrm{U}(\mathrm{eq})$ \\
\hline$\overline{H(1)}$ & $5350(15)$ & $8860(30)$ & $10160(30)$ & $45(9)$ \\
\hline $\mathrm{H}(2)$ & $5776(13)$ & $7370(30)$ & $12120(20)$ & $24(7)$ \\
\hline $\mathrm{H}(31)$ & $6852(18)$ & $7980(30)$ & $11640(30)$ & $56(11)$ \\
\hline $\mathrm{H}(32)$ & $6529(18)$ & $8920(40)$ & $10750(30)$ & $72(12)$ \\
\hline $\mathrm{H}(4)$ & $6779(15)$ & $5920(30)$ & $10690(30)$ & $46(9)$ \\
\hline $\mathrm{H}(51)$ & $5705(18)$ & $7190(40)$ & $9080(30)$ & $65(12)$ \\
\hline $\mathrm{H}(52)$ & $5807(15)$ & $5690(30)$ & $9330(30)$ & $46(10)$ \\
\hline $\mathrm{H}(6)$ & $5143(13)$ & $5990(30)$ & $10660(20)$ & $26(7)$ \\
\hline $\mathrm{H}(91)$ & $3909(14)$ & $9470(30)$ & $12650(20)$ & $33(8)$ \\
\hline $\mathrm{H}(92)$ & $4400(20)$ & $8640(40)$ & $13580(40)$ & $93(15)$ \\
\hline $\mathrm{H}(93)$ & $3600(20)$ & $8600(40)$ & $13470(30)$ & $71(12)$ \\
\hline $\mathrm{H}(101)$ & $4380(19)$ & $6020(40)$ & $13360(30)$ & $62(12)$ \\
\hline $\mathrm{H}(102)$ & $3639(18)$ & $6020(30)$ & $13370(30)$ & $57(11)$ \\
\hline $\mathrm{H}(103)$ & $3976(17)$ & $5330(40)$ & $12420(30)$ & $64(11)$ \\
\hline $\mathrm{H}(111)$ & $2877(16)$ & $7320(30)$ & $11870(20)$ & $40(9)$ \\
\hline $\mathrm{H}(112)$ & $3233(17)$ & $6660(40)$ & $10940(30)$ & $56(10)$ \\
\hline $\mathrm{H}(113)$ & $3160(20)$ & $8280(40)$ & $11020(40)$ & $84(14)$ \\
\hline $\mathrm{H}(13)$ & $6728(16)$ & $9050(30)$ & $9030(30)$ & $46(9)$ \\
\hline $\mathrm{H}(14)$ & $7559(14)$ & $9520(30)$ & $7960(20)$ & 41(9) \\
\hline $\mathrm{H}(16)$ & $8341(16)$ & $5840(30)$ & $8600(20)$ & $42(9)$ \\
\hline $\mathrm{H}(17)$ & $7554(14)$ & $5410(30)$ & $9670(20)$ & $30(8)$ \\
\hline $\mathrm{H}(18)$ & $8389(15)$ & $8890(30)$ & $7010(20)$ & $41(9)$ \\
\hline $\mathrm{H}(191)$ & $8333(18)$ & $6570(30)$ & $6310(30)$ & $51(10)$ \\
\hline $\mathrm{H}(192)$ & $8936(16)$ & $6130(30)$ & $7290(30)$ & $42(9)$ \\
\hline $\mathrm{H}(201)$ & $9004(17)$ & $8120(30)$ & $5580(30)$ & $59(11)$ \\
\hline $\mathrm{H}(202)$ & $9362(16)$ & $6520(30)$ & $5740(30)$ & $50(10)$ \\
\hline $\mathrm{H}(21)$ & $10090(16)$ & $7210(30)$ & $7340(20)$ & $42(9)$ \\
\hline $\mathrm{H}(221)$ & $9570(18)$ & $9770(40)$ & $7010(30)$ & $70(12)$ \\
\hline $\mathrm{H}(222)$ & $10115(17)$ & $9440(40)$ & $7960(30)$ & $57(11)$ \\
\hline $\mathrm{H}(231)$ & $9426(17)$ & $7760(30)$ & $8680(30)$ & $58(11)$ \\
\hline $\mathrm{H}(232)$ & $9107(15)$ & $9340(30)$ & $8640(30)$ & $50(10)$ \\
\hline $\mathrm{H}(241)$ & $10158(17)$ & $8920(30)$ & $5540(30)$ & $53(10)$ \\
\hline $\mathrm{H}(242)$ & $10764(18)$ & $9000(40)$ & $6510(30)$ & $65(12)$ \\
\hline $\mathrm{H}(251)$ & $10429(16)$ & $6650(30)$ & $5180(30)$ & $38(8)$ \\
\hline $\mathrm{H}(252)$ & $11041(18)$ & $6680(40)$ & $6240(30)$ & $64(11)$ \\
\hline $\mathrm{H}(261)$ & $11576(14)$ & $8370(30)$ & $5390(20)$ & $25(7)$ \\
\hline $\mathrm{H}(262)$ & $10980(20)$ & $8260(50)$ & $4310(40)$ & $104(17)$ \\
\hline $\mathrm{H}(271)$ & $11270(20)$ & $6000(40)$ & $4090(30)$ & $81(14)$ \\
\hline $\mathrm{H}(272)$ & $11860(20)$ & $6090(40)$ & $5080(30)$ & $76(13)$ \\
\hline $\mathrm{H}(281)$ & $12213(18)$ & $6410(40)$ & $3370(30)$ & $58(12)$ \\
\hline $\mathrm{H}(282)$ & $12330(30)$ & $7590(50)$ & $4040(40)$ & $120(20)$ \\
\hline $\mathrm{H}(283)$ & $11730(30)$ & $7910(60)$ & $3060(50)$ & $150(20)$ \\
\hline
\end{tabular}


Table 6. Torsion angles $\left[^{\circ}\right]$ for 3- $\{[4-($ trans $-4-n$-Pentyl)cyclohexyl]-phenyl $\}$ bicyclo[3.1.0]hexan-6carbonsäure-tertbutylester $162 \mathrm{a}$.

\begin{tabular}{|c|c|c|c|}
\hline$C(7)-C(1)-C(2)-C(6)$ & $109.9(3)$ & $\mathrm{C}(17)-\mathrm{C}(12)-\mathrm{C}(13)-\mathrm{C}(14)$ & $-1.1(5)$ \\
\hline $\mathrm{C}(7)-\mathrm{C}(1)-\mathrm{C}(2)-\mathrm{C}(3)$ & $-153.7(3)$ & $\mathrm{C}(4)-\mathrm{C}(12)-\mathrm{C}(13)-\mathrm{C}(14)$ & $179.2(3)$ \\
\hline$C(6)-C(1)-C(2)-C(3)$ & $96.4(3)$ & $\mathrm{C}(12)-\mathrm{C}(13)-\mathrm{C}(14)-\mathrm{C}(15)$ & $1.6(5)$ \\
\hline $\mathrm{C}(6)-\mathrm{C}(2)-\mathrm{C}(3)-\mathrm{C}(4)$ & $-22.1(3)$ & $\mathrm{C}(13)-\mathrm{C}(14)-\mathrm{C}(15)-\mathrm{C}(16)$ & $-0.7(4)$ \\
\hline $\mathrm{C}(1)-\mathrm{C}(2)-\mathrm{C}(3)-\mathrm{C}(4)$ & $-87.0(3)$ & $\mathrm{C}(13)-\mathrm{C}(14)-\mathrm{C}(15)-\mathrm{C}(18)$ & $-177.9(3)$ \\
\hline $\mathrm{C}(2)-\mathrm{C}(3)-\mathrm{C}(4)-\mathrm{C}(12)$ & $160.5(3)$ & $\mathrm{C}(14)-\mathrm{C}(15)-\mathrm{C}(16)-\mathrm{C}(17)$ & $-0.6(4)$ \\
\hline$C(2)-C(3)-C(4)-C(5)$ & $33.9(3)$ & $\mathrm{C}(18)-\mathrm{C}(15)-\mathrm{C}(16)-\mathrm{C}(17)$ & $176.4(3)$ \\
\hline $\mathrm{C}(12)-\mathrm{C}(4)-\mathrm{C}(5)-\mathrm{C}(6)$ & $-160.7(3)$ & $\mathrm{C}(15)-\mathrm{C}(16)-\mathrm{C}(17)-\mathrm{C}(12)$ & $1.2(5)$ \\
\hline $\mathrm{C}(3)-\mathrm{C}(4)-\mathrm{C}(5)-\mathrm{C}(6)$ & $-33.2(3)$ & $\mathrm{C}(13)-\mathrm{C}(12)-\mathrm{C}(17)-\mathrm{C}(16)$ & $-0.3(5)$ \\
\hline$C(3)-C(2)-C(6)-C(1)$ & $-109.8(3)$ & $C(4)-C(12)-C(17)-C(16)$ & $179.4(3)$ \\
\hline$C(1)-C(2)-C(6)-C(5)$ & $111.0(3)$ & $\mathrm{C}(16)-\mathrm{C}(15)-\mathrm{C}(18)-\mathrm{C}(19)$ & $40.5(4)$ \\
\hline $\mathrm{C}(3)-\mathrm{C}(2)-\mathrm{C}(6)-\mathrm{C}(5)$ & $1.2(3)$ & $\mathrm{C}(14)-\mathrm{C}(15)-\mathrm{C}(18)-\mathrm{C}(19)$ & $-142.5(3)$ \\
\hline$C(7)-C(1)-C(6)-C(2)$ & $-108.5(3)$ & $C(16)-C(15)-C(18)-C(23)$ & $-83.8(4)$ \\
\hline$C(7)-C(1)-C(6)-C(5)$ & $154.9(3)$ & $\mathrm{C}(14)-\mathrm{C}(15)-\mathrm{C}(18)-\mathrm{C}(23)$ & $93.2(3)$ \\
\hline$C(2)-C(1)-C(6)-C(5)$ & $-96.5(3)$ & $\mathrm{C}(15)-\mathrm{C}(18)-\mathrm{C}(19)-\mathrm{C}(20)$ & $-179.7(3)$ \\
\hline$C(4)-C(5)-C(6)-C(2)$ & $19.8(3)$ & $\mathrm{C}(23)-\mathrm{C}(18)-\mathrm{C}(19)-\mathrm{C}(20)$ & $-54.7(4)$ \\
\hline$C(4)-C(5)-C(6)-C(1)$ & $85.1(3)$ & $\mathrm{C}(18)-\mathrm{C}(19)-\mathrm{C}(20)-\mathrm{C}(21)$ & $54.6(4)$ \\
\hline $\mathrm{C}(8)-\mathrm{O}(1)-\mathrm{C}(7)-\mathrm{O}(2)$ & $-0.1(4)$ & $C(19)-C(20)-C(21)-C(22)$ & $-52.7(4)$ \\
\hline $\mathrm{C}(8)-\mathrm{O}(1)-\mathrm{C}(7)-\mathrm{C}(1)$ & $-179.8(2)$ & $C(19)-C(20)-C(21)-C(24)$ & $-177.4(3)$ \\
\hline $\mathrm{C}(2)-\mathrm{C}(1)-\mathrm{C}(7)-\mathrm{O}(2)$ & $140.3(3)$ & $\mathrm{C}(24)-\mathrm{C}(21)-\mathrm{C}(22)-\mathrm{C}(23)$ & 179.1(3) \\
\hline $\mathrm{C}(6)-\mathrm{C}(1)-\mathrm{C}(7)-\mathrm{O}(2)$ & $-150.0(3)$ & $\mathrm{C}(20)-\mathrm{C}(21)-\mathrm{C}(22)-\mathrm{C}(23)$ & $53.9(4)$ \\
\hline $\mathrm{C}(2)-\mathrm{C}(1)-\mathrm{C}(7)-\mathrm{O}(1)$ & $-40.0(4)$ & $\mathrm{C}(21)-\mathrm{C}(22)-\mathrm{C}(23)-\mathrm{C}(18)$ & $-57.2(4)$ \\
\hline $\mathrm{C}(6)-\mathrm{C}(1)-\mathrm{C}(7)-\mathrm{O}(1)$ & $29.7(4)$ & $\mathrm{C}(15)-\mathrm{C}(18)-\mathrm{C}(23)-\mathrm{C}(22)$ & $-176.9(3)$ \\
\hline $\mathrm{C}(7)-\mathrm{O}(1)-\mathrm{C}(8)-\mathrm{C}(10)$ & $178.9(3)$ & $\mathrm{C}(19)-\mathrm{C}(18)-\mathrm{C}(23)-\mathrm{C}(22)$ & $55.9(4)$ \\
\hline $\mathrm{C}(7)-\mathrm{O}(1)-\mathrm{C}(8)-\mathrm{C}(11)$ & $61.2(3)$ & $C(22)-C(21)-C(24)-C(25)$ & $164.7(3)$ \\
\hline $\mathrm{C}(7)-\mathrm{O}(1)-\mathrm{C}(8)-\mathrm{C}(9)$ & $-63.2(3)$ & $\mathrm{C}(20)-\mathrm{C}(21)-\mathrm{C}(24)-\mathrm{C}(25)$ & $-71.7(4)$ \\
\hline $\mathrm{C}(3)-\mathrm{C}(4)-\mathrm{C}(12)-\mathrm{C}(13)$ & $-47.4(4)$ & $\mathrm{C}(21)-\mathrm{C}(24)-\mathrm{C}(25)-\mathrm{C}(26)$ & $177.4(3)$ \\
\hline$C(5)-C(4)-C(12)-C(13)$ & $73.6(4)$ & $\mathrm{C}(24)-\mathrm{C}(25)-\mathrm{C}(26)-\mathrm{C}(27)$ & $178.9(3)$ \\
\hline$C(3)-C(4)-C(12)-C(17)$ & $132.9(3)$ & $\mathrm{C}(25)-\mathrm{C}(26)-\mathrm{C}(27)-\mathrm{C}(28)$ & $176.2(4)$ \\
\hline$C(5)-C(4)-C(12)-C(17)$ & $-106.1(3)$ & & \\
\hline
\end{tabular}


Table 1. Crystal data and structure refinement for exo,exo-3-(3,4,5-Trifluorophenyl)bicyclo[3.1.0]hexane-6carboxylic acid-tert-butylester $\mathbf{1 6 2 b}$.

Identification code: exo,exo-3-(3,4,5-Trifluorophenyl)bicyclo[3.1.0]hexan-6-carbonsäure-tert-butylester 162b

Empirical formula

Formula weight

Temperature

Wavelength

Crystal system

Space group

Unit cell dimensions

Volume

Z

Density (calculated)

Absorption coefficient

$\mathrm{F}(000)$

Crystal size

Theta range for data collection

Index ranges

Reflections collected
$\mathrm{C}_{17} \mathrm{H}_{19} \mathrm{~F}_{3} \mathrm{O}_{2}$

312.32

120.0(2) K

$0.71073 \AA$

Triclinic

P -1

$\mathrm{a}=5.7364(3) \AA$

$\alpha=76.584(2)^{\circ}$.

$\mathrm{b}=11.0353(6) \AA$

$\beta=87.759(2)^{\circ}$.

$\mathrm{c}=12.8208(7) \AA$

$\gamma=87.674(2)^{\circ}$.

2

$1.316 \mathrm{Mg} / \mathrm{m}^{3}$

$0.108 \mathrm{~mm}^{-1}$

328

$0.36 \times 0.21 \times 0.16 \mathrm{~mm}^{3}$

1.90 to $30.17^{\circ}$.

$-7<=\mathrm{h}<=8,-15<=\mathrm{k}<=15,-16<=\mathrm{l}<=18$

8036 
Independent reflections

Completeness to theta $=30.17^{\circ}$

Absorption correction

Max. and min. transmission

Refinement method

Data / restraints / parameters

Goodness-of-fit on $\mathrm{F}^{2}$

Final $\mathrm{R}$ indices $[\mathrm{I}>2 \operatorname{sigma}(\mathrm{I})]$

$\mathrm{R}$ indices (all data)

Largest diff. peak and hole
$4178[\mathrm{R}(\mathrm{int})=0.0295]$

$89.6 \%$

None

0.9829 and 0.9621

Full-matrix least-squares on $\mathrm{F}^{2}$

4178 / 0 / 275

1.039

$\mathrm{R} 1=0.0434, \mathrm{wR} 2=0.1096$

$\mathrm{R} 1=0.0573, \mathrm{wR} 2=0.1191$

0.347 and -0.207 e. $\AA^{-3}$ 
Table 2. Atomic coordinates ( $\left.\mathrm{x} 10^{4}\right)$ and equivalent isotropic displacement parameters $\left(\AA^{2} \times 10^{3}\right)$ for exo,exo-3-(3,4,5-Trifluorophenyl)bicyclo[3.1.0]hexan-6-carbonsäure-tert-butylester 162b. U(eq) is defined as one third of the trace of the orthogonalized $U^{i j}$ tensor.

\begin{tabular}{|c|c|c|c|c|}
\hline Atom & $\mathrm{x}$ & $\mathrm{y}$ & z & $\mathrm{U}(\mathrm{eq})$ \\
\hline $\mathrm{O}(1)$ & $2333(2)$ & 2361(1) & $6565(1)$ & $23(1)$ \\
\hline $\mathrm{O}(2)$ & $-398(2)$ & $1489(1)$ & 5751(1) & $20(1)$ \\
\hline $\mathrm{F}(1)$ & $1707(2)$ & $6651(1)$ & $-1049(1)$ & $36(1)$ \\
\hline $\mathrm{F}(2)$ & $5487(2)$ & $8088(1)$ & $-1184(1)$ & $38(1)$ \\
\hline $\mathrm{F}(3)$ & $8194(2)$ & $7860(1)$ & $535(1)$ & $37(1)$ \\
\hline $\mathrm{C}(1)$ & $1812(2)$ & $3045(1)$ & $4675(1)$ & $18(1)$ \\
\hline $\mathrm{C}(2)$ & 2693(2) & $4342(1)$ & $4618(1)$ & $18(1)$ \\
\hline$C(3)$ & $2372(2)$ & $5300(1)$ & $3570(1)$ & $20(1)$ \\
\hline $\mathrm{C}(4)$ & $3218(2)$ & $4623(1)$ & $2683(1)$ & $19(1)$ \\
\hline$C(5)$ & $5132(2)$ & $3683(1)$ & $3226(1)$ & $21(1)$ \\
\hline $\mathrm{C}(6)$ & $4380(2)$ & $3347(1)$ & $4406(1)$ & $19(1)$ \\
\hline$C(7)$ & $1310(2)$ & $2277(1)$ & $5774(1)$ & $18(1)$ \\
\hline $\mathrm{C}(8)$ & $-1162(2)$ & $547(1)$ & $6723(1)$ & $20(1)$ \\
\hline$C(9)$ & $871(3)$ & $-343(1)$ & $7134(1)$ & $31(1)$ \\
\hline $\mathrm{C}(10)$ & $-3014(2)$ & $-118(1)$ & $6270(1)$ & $25(1)$ \\
\hline $\mathrm{C}(11)$ & $-2211(3)$ & $1184(1)$ & $7576(1)$ & $25(1)$ \\
\hline $\mathrm{C}(12)$ & $3917(2)$ & $5519(1)$ & $1643(1)$ & $19(1)$ \\
\hline $\mathrm{C}(13)$ & $2520(2)$ & $5650(1)$ & $752(1)$ & $22(1)$ \\
\hline$C(14)$ & $3063(2)$ & $6514(1)$ & $-185(1)$ & $24(1)$ \\
\hline$C(15)$ & 4965(3) & $7254(1)$ & $-265(1)$ & $25(1)$ \\
\hline$C(16)$ & $6347(2)$ & $7120(1)$ & $622(1)$ & $24(1)$ \\
\hline$C(17)$ & $5852(2)$ & $6269(1)$ & $1572(1)$ & $22(1)$ \\
\hline
\end{tabular}


Table 3. Bond lengths $[\AA]$ and angles $\left[^{\circ}\right]$ for exo,exo-3-(3,4,5-Trifluorophenyl)bicyclo[3.1.0]hexane-6carboxylic acid tert-butylester $\mathbf{1 6 2 b}$.

\begin{tabular}{|l|l|l|l|l|r|}
\hline $\mathrm{O}(1)-\mathrm{C}(7)$ & $1.215(2)$ & $\mathrm{C}(1)-\mathrm{C}(6)$ & $1.532(2)$ & $\mathrm{C}(8)-\mathrm{C}(9)$ & $1.522(2)$ \\
\hline $\mathrm{O}(2)-\mathrm{C}(7)$ & $1.341(2)$ & $\mathrm{C}(2)-\mathrm{C}(6)$ & $1.499(2)$ & $\mathrm{C}(8)-\mathrm{C}(11)$ & $1.524(2)$ \\
\hline $\mathrm{O}(2)-\mathrm{C}(8)$ & $1.490(1)$ & $\mathrm{C}(2)-\mathrm{C}(3)$ & $1.517(2)$ & $\mathrm{C}(12)-\mathrm{C}(13)$ & $1.397(2)$ \\
\hline $\mathrm{F}(1)-\mathrm{C}(14)$ & $1.355(2)$ & $\mathrm{C}(3)-\mathrm{C}(4)$ & $1.552(2)$ & $\mathrm{C}(12)-\mathrm{C}(17)$ & $1.399(2)$ \\
\hline $\mathrm{F}(2)-\mathrm{C}(15)$ & $1.347(1)$ & $\mathrm{C}(4)-\mathrm{C}(12)$ & $1.515(2)$ & $\mathrm{C}(13)-\mathrm{C}(14)$ & $1.384(2)$ \\
\hline $\mathrm{F}(3)-\mathrm{C}(16)$ & $1.349(2)$ & $\mathrm{C}(4)-\mathrm{C}(5)$ & $1.549(2)$ & $\mathrm{C}(14)-\mathrm{C}(15)$ & $1.376(2)$ \\
\hline $\mathrm{C}(1)-\mathrm{C}(7)$ & $1.490(2)$ & $\mathrm{C}(5)-\mathrm{C}(6)$ & $1.521(2)$ & $\mathrm{C}(15)-\mathrm{C}(16)$ & $1.388(2)$ \\
\hline $\mathrm{C}(1)-\mathrm{C}(2)$ & $1.522(2)$ & $\mathrm{C}(8)-\mathrm{C}(10)$ & $1.521(2)$ & $\mathrm{C}(16)-\mathrm{C}(17)$ & $1.382(2)$ \\
\hline
\end{tabular}

\begin{tabular}{|l|l|l|l|}
\hline $\mathrm{C}(7)-\mathrm{O}(2)-\mathrm{C}(8)$ & $122.09(9)$ & $\mathrm{O}(2)-\mathrm{C}(8)-\mathrm{C}(9)$ & $109.9(1)$ \\
\hline $\mathrm{C}(7)-\mathrm{C}(1)-\mathrm{C}(2)$ & $115.8(1)$ & $\mathrm{C}(10)-\mathrm{C}(8)-\mathrm{C}(9)$ & $110.8(1)$ \\
\hline $\mathrm{C}(7)-\mathrm{C}(1)-\mathrm{C}(6)$ & $115.6(1)$ & $\mathrm{O}(2)-\mathrm{C}(8)-\mathrm{C}(11)$ & $110.6(1)$ \\
\hline $\mathrm{C}(2)-\mathrm{C}(1)-\mathrm{C}(6)$ & $58.80(8)$ & $\mathrm{C}(10)-\mathrm{C}(8)-\mathrm{C}(11)$ & $111.2(1)$ \\
\hline $\mathrm{C}(6)-\mathrm{C}(2)-\mathrm{C}(3)$ & $107.8(1)$ & $\mathrm{C}(9)-\mathrm{C}(8)-\mathrm{C}(11)$ & $112.5(1)$ \\
\hline $\mathrm{C}(6)-\mathrm{C}(2)-\mathrm{C}(1)$ & $60.94(8)$ & $\mathrm{C}(13)-\mathrm{C}(12)-\mathrm{C}(17)$ & $119.3(1)$ \\
\hline $\mathrm{C}(3)-\mathrm{C}(2)-\mathrm{C}(1)$ & $116.7(1)$ & $\mathrm{C}(13)-\mathrm{C}(12)-\mathrm{C}(4)$ & $119.2(1)$ \\
\hline $\mathrm{C}(2)-\mathrm{C}(3)-\mathrm{C}(4)$ & $105.1(1)$ & $\mathrm{C}(17)-\mathrm{C}(12)-\mathrm{C}(4)$ & $121.5(1)$ \\
\hline $\mathrm{C}(12)-\mathrm{C}(4)-\mathrm{C}(5)$ & $116.6(1)$ & $\mathrm{C}(14)-\mathrm{C}(13)-\mathrm{C}(12)$ & $119.8(1)$ \\
\hline $\mathrm{C}(12)-\mathrm{C}(4)-\mathrm{C}(3)$ & $112.7(1)$ & $\mathrm{F}(1)-\mathrm{C}(14)-\mathrm{C}(15)$ & $118.5(1)$ \\
\hline $\mathrm{C}(5)-\mathrm{C}(4)-\mathrm{C}(3)$ & $104.0(1)$ & $\mathrm{F}(1)-\mathrm{C}(14)-\mathrm{C}(13)$ & $120.1(1)$ \\
\hline $\mathrm{C}(6)-\mathrm{C}(5)-\mathrm{C}(4)$ & $104.6(1)$ & $\mathrm{C}(15)-\mathrm{C}(14)-\mathrm{C}(13)$ & $121.4(1)$ \\
\hline $\mathrm{C}(2)-\mathrm{C}(6)-\mathrm{C}(5)$ & $108.4(1)$ & $\mathrm{F}(2)-\mathrm{C}(15)-\mathrm{C}(14)$ & $120.9(1)$ \\
\hline $\mathrm{C}(2)-\mathrm{C}(6)-\mathrm{C}(1)$ & $60.26(8)$ & $\mathrm{F}(2)-\mathrm{C}(15)-\mathrm{C}(16)$ & $120.6(1)$ \\
\hline $\mathrm{C}(5)-\mathrm{C}(6)-\mathrm{C}(1)$ & $117.4(1)$ & $\mathrm{C}(14)-\mathrm{C}(15)-\mathrm{C}(16)$ & $118.5(1)$ \\
\hline $\mathrm{O}(1)-\mathrm{C}(7)-\mathrm{O}(2)$ & $125.8(1)$ & $\mathrm{F}(3)-\mathrm{C}(16)-\mathrm{C}(17)$ & $120.5(1)$ \\
\hline $\mathrm{O}(1)-\mathrm{C}(7)-\mathrm{C}(1)$ & $124.0(1)$ & $\mathrm{F}(3)-\mathrm{C}(16)-\mathrm{C}(15)$ & $117.9(1)$ \\
\hline $\mathrm{O}(2)-\mathrm{C}(7)-\mathrm{C}(1)$ & $110.2(1)$ & $\mathrm{C}(17)-\mathrm{C}(16)-\mathrm{C}(15)$ & $121.6(1)$ \\
\hline $\mathrm{O}(2)-\mathrm{C}(8)-\mathrm{C}(10)$ & $101.4(1)$ & $\mathrm{C}(16)-\mathrm{C}(17)-\mathrm{C}(12)$ & $119.4(1)$ \\
\hline
\end{tabular}


Table 4. Anisotropic displacement parameters $\left(\AA^{2} \times 10^{3}\right)$ for exo,exo-3-(3,4,5-

Trifluorophenyl)bicyclo[3.1.0]hexane-6-carboxylic acid tert-butylester 162b. The anisotropic displacement factor exponent takes the form: $-2 \pi^{2}\left[h^{2} a^{* 2} U^{11}+\ldots+2 h k a^{*} b^{*} U^{12}\right]$

\begin{tabular}{|c|c|c|c|c|c|c|}
\hline Atom & $\mathrm{U}^{11}$ & $\mathrm{U}^{22}$ & $\mathrm{U}^{33}$ & $\mathrm{U}^{23}$ & $\mathrm{U}^{13}$ & $\mathrm{U}^{12}$ \\
\hline $\mathrm{O}(1)$ & $23(1)$ & $25(1)$ & $20(1)$ & $-1(1)$ & $-2(1)$ & $-5(1)$ \\
\hline $\mathrm{O}(2)$ & 21(1) & $19(1)$ & $18(1)$ & $0(1)$ & 1(1) & $-6(1)$ \\
\hline $\mathrm{F}(1)$ & $43(1)$ & $41(1)$ & $20(1)$ & $3(1)$ & $-11(1)$ & $-9(1)$ \\
\hline $\mathrm{F}(2)$ & $51(1)$ & $34(1)$ & 21(1) & $7(1)$ & $2(1)$ & $-14(1)$ \\
\hline $\mathrm{F}(3)$ & $37(1)$ & $38(1)$ & $32(1)$ & $0(1)$ & 2(1) & $-22(1)$ \\
\hline $\mathrm{C}(1)$ & 19(1) & $17(1)$ & $18(1)$ & $-2(1)$ & $0(1)$ & $-4(1)$ \\
\hline$C(2)$ & $21(1)$ & $16(1)$ & $16(1)$ & $-3(1)$ & 2(1) & $-4(1)$ \\
\hline $\mathrm{C}(3)$ & $23(1)$ & $17(1)$ & 19(1) & $-1(1)$ & $3(1)$ & $0(1)$ \\
\hline C(4) & $22(1)$ & $18(1)$ & $16(1)$ & $-2(1)$ & $0(1)$ & $-5(1)$ \\
\hline $\mathrm{C}(5)$ & $23(1)$ & $18(1)$ & 19(1) & $-2(1)$ & $4(1)$ & $-1(1)$ \\
\hline $\mathrm{C}(6)$ & $18(1)$ & $19(1)$ & $18(1)$ & $-1(1)$ & 1(1) & $-2(1)$ \\
\hline$C(7)$ & $17(1)$ & $16(1)$ & $20(1)$ & $-2(1)$ & 1(1) & $-2(1)$ \\
\hline$C(8)$ & $20(1)$ & $17(1)$ & $20(1)$ & $2(1)$ & $4(1)$ & $-3(1)$ \\
\hline $\mathrm{C}(9)$ & $24(1)$ & $24(1)$ & $39(1)$ & $6(1)$ & $3(1)$ & $2(1)$ \\
\hline$C(10)$ & $25(1)$ & $22(1)$ & $28(1)$ & $-5(1)$ & $5(1)$ & $-8(1)$ \\
\hline $\mathrm{C}(11)$ & $27(1)$ & $25(1)$ & 21(1) & $-3(1)$ & $4(1)$ & $-5(1)$ \\
\hline $\mathrm{C}(12)$ & $22(1)$ & $17(1)$ & $17(1)$ & $-3(1)$ & $2(1)$ & $-1(1)$ \\
\hline$C(13)$ & $24(1)$ & $21(1)$ & $20(1)$ & $-4(1)$ & $0(1)$ & $-4(1)$ \\
\hline$C(14)$ & $30(1)$ & $25(1)$ & $16(1)$ & $-2(1)$ & $-3(1)$ & $0(1)$ \\
\hline$C(15)$ & $33(1)$ & $20(1)$ & $18(1)$ & $0(1)$ & $4(1)$ & $-4(1)$ \\
\hline$C(16)$ & $26(1)$ & $22(1)$ & $23(1)$ & $-5(1)$ & $4(1)$ & $-8(1)$ \\
\hline$C(17)$ & $23(1)$ & $23(1)$ & 19(1) & $-4(1)$ & $0(1)$ & $-5(1)$ \\
\hline
\end{tabular}


Table 5. Hydrogen coordinates $\left(\times 10^{4}\right)$ and isotropic displacement parameters $\left(\AA^{2} \times 10^{3}\right)$ for exo,exo-3-(3,4,5Trifluorophenyl)bicyclo[3.1.0]hexane-6-carboxylic acid tert-butylester $\mathbf{1 6 2 b}$.

\begin{tabular}{|c|c|c|c|c|}
\hline Atom & $\mathrm{x}$ & $\mathrm{y}$ & $\mathrm{z}$ & $\mathrm{U}(\mathrm{eq})$ \\
\hline $\mathrm{H}(1)$ & $800(30)$ & $2906(13)$ & $4141(12)$ & $16(3)$ \\
\hline $\mathrm{H}(2)$ & $2640(30)$ & $4612(15)$ & $5289(13)$ & $23(4)$ \\
\hline $\mathrm{H}(31)$ & $3360(30)$ & $6016(15)$ & $3558(13)$ & $24(4)$ \\
\hline $\mathrm{H}(32)$ & $710(30)$ & $5613(16)$ & $3477(13)$ & $28(4)$ \\
\hline $\mathrm{H}(4)$ & $1890(30)$ & $4140(15)$ & $2517(13)$ & $24(4)$ \\
\hline $\mathrm{H}(51)$ & $5330(30)$ & $2961(15)$ & $2900(13)$ & $22(4)$ \\
\hline $\mathrm{H}(52)$ & $6670(30)$ & $4118(16)$ & $3167(14)$ & $27(4)$ \\
\hline $\mathrm{H}(6)$ & $5500(30)$ & $2953(15)$ & 4919(13) & $27(4)$ \\
\hline $\mathrm{H}(91)$ & $210(30)$ & $-1012(18)$ & $7715(15)$ & $38(5)$ \\
\hline $\mathrm{H}(92)$ & $2040(30)$ & $65(17)$ & $7442(14)$ & $31(4)$ \\
\hline $\mathrm{H}(93)$ & $1600(40)$ & $-688(19)$ & $6540(17)$ & $48(6)$ \\
\hline $\mathrm{H}(101)$ & $-4310(30)$ & $455(17)$ & $5956(14)$ & $31(4)$ \\
\hline $\mathrm{H}(102)$ & $-2340(30)$ & $-486(16)$ & $5696(15)$ & $32(4)$ \\
\hline $\mathrm{H}(103)$ & $-3650(30)$ & $-802(17)$ & $6872(15)$ & $37(5)$ \\
\hline $\mathrm{H}(111)$ & $-1040(30)$ & $1660(17)$ & $7852(15)$ & $36(5)$ \\
\hline $\mathrm{H}(112)$ & $-3510(30)$ & $1736(17)$ & $7269(15)$ & $36(5)$ \\
\hline $\mathrm{H}(113)$ & $-2820(30)$ & $527(16)$ & $8188(14)$ & $29(4)$ \\
\hline $\mathrm{H}(13)$ & $1140(30)$ & $5149(16)$ & $781(14)$ & $29(4)$ \\
\hline $\mathrm{H}(17)$ & $6860(30)$ & $6246(16)$ & $2163(14)$ & $32(4)$ \\
\hline
\end{tabular}


Table 6. Torsion angles $\left[{ }^{\circ}\right]$ for exo,exo-3-(3,4,5-Trifluorophenyl)bicyclo[3.1.0]hexane-6-carboxylic acid tertbutylester 162b.

\begin{tabular}{|l|l|l|l|}
\hline$C(7)-C(1)-C(2)-C(6)$ & $105.58(12)$ & $C(7)-O(2)-C(8)-C(10)$ & $-177.93(11)$ \\
\hline$C(7)-C(1)-C(2)-C(3)$ & $-157.97(11)$ & $C(7)-O(2)-C(8)-C(9)$ & $-60.70(15)$ \\
\hline$C(6)-C(1)-C(2)-C(3)$ & $96.45(12)$ & $C(7)-O(2)-C(8)-C(11)$ & $64.07(14)$ \\
\hline$C(6)-C(2)-C(3)-C(4)$ & $18.98(13)$ & $C(5)-C(4)-C(12)-C(13)$ & $129.95(13)$ \\
\hline$C(1)-C(2)-C(3)-C(4)$ & $-46.85(14)$ & $C(3)-C(4)-C(12)-C(13)$ & $-109.90(13)$ \\
\hline$C(2)-C(3)-C(4)-C(12)$ & $-157.80(10)$ & $C(5)-C(4)-C(12)-C(17)$ & $-53.81(16)$ \\
\hline$C(2)-C(3)-C(4)-C(5)$ & $-30.58(12)$ & $C(3)-C(4)-C(12)-C(17)$ & $66.34(15)$ \\
\hline$C(12)-C(4)-C(5)-C(6)$ & $155.35(11)$ & $C(17)-C(12)-C(13)-C(14)$ & $0.09(19)$ \\
\hline$C(3)-C(4)-C(5)-C(6)$ & $30.63(12)$ & $C(4)-C(12)-C(13)-C(14)$ & $176.41(12)$ \\
\hline$C(3)-C(2)-C(6)-C(5)$ & $0.36(14)$ & $C(12)-C(13)-C(14)-F(1)$ & $-179.57(12)$ \\
\hline$C(1)-C(2)-C(6)-C(5)$ & $111.58(11)$ & $C(12)-C(13)-C(14)-C(15)$ & $0.1(2)$ \\
\hline$C(3)-C(2)-C(6)-C(1)$ & $-111.22(11)$ & $F(1)-C(14)-C(15)-F(2)$ & $-0.7(2)$ \\
\hline$C(4)-C(5)-C(6)-C(2)$ & $-19.55(13)$ & $C(13)-C(14)-C(15)-F(2)$ & $179.57(12)$ \\
\hline$C(4)-C(5)-C(6)-C(1)$ & $45.82(14)$ & $F(1)-C(14)-C(15)-C(16)$ & $179.59(12)$ \\
\hline$C(7)-C(1)-C(6)-C(2)$ & $-105.88(12)$ & $C(13)-C(14)-C(15)-C(16)$ & $-0.1(2)$ \\
\hline$C(7)-C(1)-C(6)-C(5)$ & $157.63(11)$ & $F(2)-C(15)-C(16)-F(3)$ & $0.9(2)$ \\
\hline$C(2)-C(1)-C(6)-C(5)$ & $-96.49(12)$ & $C(14)-C(15)-C(16)-F(3)$ & $-179.42(12)$ \\
\hline$C(8)-O(2)-C(7)-O(1)$ & $-3.62(19)$ & $F(2)-C(15)-C(16)-C(17)$ & $-179.80(12)$ \\
\hline$C(8)-O(2)-C(7)-C(1)$ & $175.94(10)$ & $C(14)-C(15)-C(16)-C(17)$ & $-0.1(2)$ \\
\hline$C(2)-C(1)-C(7)-O(1)$ & $-30.21(17)$ & $F(3)-C(16)-C(17)-C(12)$ & $179.61(12)$ \\
\hline$C(6)-C(1)-C(7)-O(1)$ & $35.81(17)$ & $C(15)-C(16)-C(17)-C(12)$ & $0.3(2)$ \\
\hline$C(2)-C(1)-C(7)-O(2)$ & $150.22(11)$ & $C(13)-C(12)-C(17)-C(16)$ & $-0.31(19)$ \\
\hline$C(6)-C(1)-C(7)-O(2)$ & $-143.76(11)$ & $-176.55(12)-C(17)-C(16)$ & \\
\hline
\end{tabular}


4.4. endo,exo-6-(4-Cyanophenyl)-3-n-pentylbicyclo[3.1.0]hexan 155b

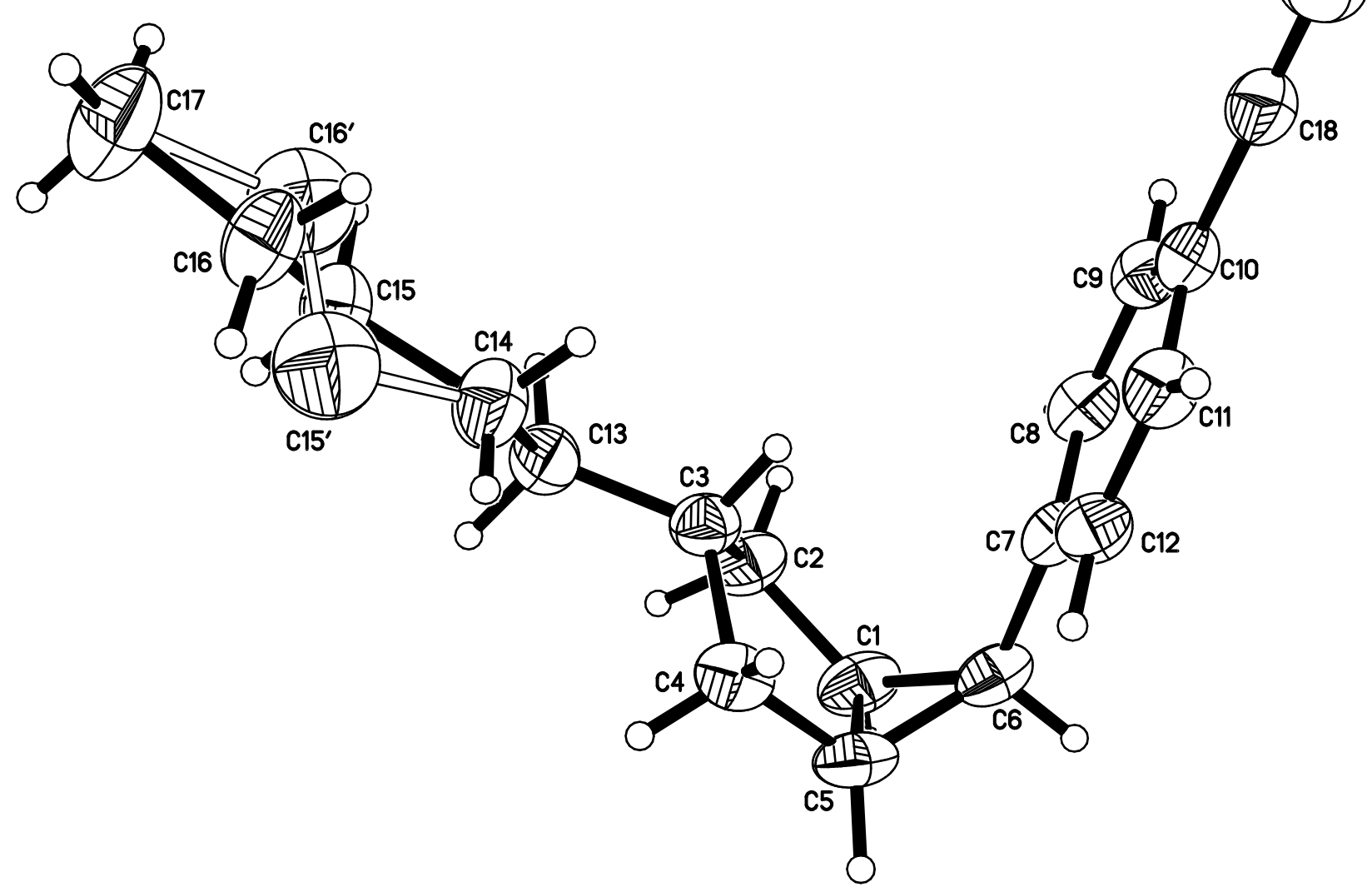

Table 1. Crystal data and structure refinement for endo,exo-6-(4-Cyanophenyl)-3-n-

pentylbicyclo[3.1.0]hexan 155b.

Identification code: endo,exo-6-(4-Cyanophenyl)-3-n-pentylbicyclo[3.1.0]hexan 155b

Empirical formula

Formula weight

Temperature

Wavelength

Crystal system

Space group

Unit cell dimensions

Volume

$\mathrm{Z}$

Density (calculated)

Absorption coefficient

$\mathrm{F}(000)$

Crystal size

Theta range for data collection
$\mathrm{C}_{18} \mathrm{H}_{23} \mathrm{~N}$

253.37

$100.0(2) \mathrm{K}$

$0.71073 \AA$

Triclinic

$\mathrm{P}-1$

$\mathrm{a}=6.0817(5) \AA$

$\alpha=81.97(3)^{\circ}$.

$\mathrm{b}=8.5321(7) \AA$

$\beta=85.25(3)^{\circ}$.

$\mathrm{c}=15.048(3) \AA$

$\gamma=87.91(3)^{\circ}$.

$770.29(18) \AA^{3}$

2

$1.092 \mathrm{Mg} / \mathrm{m}^{3}$

$0.063 \mathrm{~mm}^{-1}$

276

$0.40 \times 0.25 \times 0.20 \mathrm{~mm}^{3}$

2.41 to $30.55^{\circ}$. 
Index ranges

Reflections collected

Independent reflections

Completeness to theta $=30.55^{\circ}$

Absorption correction

Max. and min. transmission

Refinement method

Data / restraints / parameters

Goodness-of-fit on $\mathrm{F}^{2}$

Final R indices $[\mathrm{I}>2 \operatorname{sigma}(\mathrm{I})]$

$\mathrm{R}$ indices (all data)

Largest diff. peak and hole $-8<=\mathrm{h}<=8,-12<=\mathrm{k}<=11,-21<=\mathrm{l}<=19$

6797

$4553[\mathrm{R}($ int $)=0.0303]$

$96.1 \%$

None

0.9876 and 0.9754

Full-matrix least-squares on $\mathrm{F}^{2}$

4553 / 0 / 272

0.948

$\mathrm{R} 1=0.0487, \mathrm{wR} 2=0.1249$

$\mathrm{R} 1=0.0893, \mathrm{wR} 2=0.1401$

0.236 and -0.149 e. $\AA^{-3}$ 
Table 2. Atomic coordinates $\left(\mathrm{x} 10^{4}\right)$ and equivalent isotropic displacement parameters $\left(\AA^{2} \times 10^{3}\right)$ for endo,exo-6-(4-Cyanophenyl)-3-n-pentylbicyclo[3.1.0]hexan 155b. U(eq) is defined as one third of the trace of the orthogonalized $\mathrm{U}^{\mathrm{ij}}$ tensor.

\begin{tabular}{|c|c|c|c|c|}
\hline Atom & $\mathrm{x}$ & $\mathrm{y}$ & $\mathrm{z}$ & $\mathrm{U}(\mathrm{eq})$ \\
\hline $\mathrm{N}(1)$ & $13865(2)$ & $6990(2)$ & $4227(1)$ & $68(1)$ \\
\hline $\mathrm{C}(1)$ & $7982(2)$ & $-508(2)$ & $3151(1)$ & $56(1)$ \\
\hline $\mathrm{C}(2)$ & $10105(2)$ & $-549(2)$ & $2566(1)$ & $54(1)$ \\
\hline $\mathrm{C}(3)$ & $10174(2)$ & $1010(2)$ & 1932(1) & $47(1)$ \\
\hline$C(4)$ & $7736(2)$ & $1511(2)$ & $1850(1)$ & $55(1)$ \\
\hline$C(5)$ & $6499(2)$ & $754(2)$ & $2703(1)$ & $56(1)$ \\
\hline$C(6)$ & $7341(2)$ & $933(2)$ & $3591(1)$ & $51(1)$ \\
\hline$C(7)$ & $8834(2)$ & $2208(1)$ & $3718(1)$ & $43(1)$ \\
\hline $\mathrm{C}(8)$ & $10773(2)$ & $1890(2)$ & $4144(1)$ & $48(1)$ \\
\hline $\mathrm{C}(9)$ & $12097(2)$ & $3096(2)$ & $4284(1)$ & $50(1)$ \\
\hline$C(10)$ & $11487(2)$ & $4654(2)$ & $3995(1)$ & $45(1)$ \\
\hline $\mathrm{C}(11)$ & $9548(2)$ & 4994(2) & $3571(1)$ & $50(1)$ \\
\hline$C(12)$ & $8239(2)$ & $3779(2)$ & $3444(1)$ & $48(1)$ \\
\hline$C(13)$ & $11527(2)$ & $899(2)$ & $1049(1)$ & $55(1)$ \\
\hline$C(14)$ & 11733(3) & $2443(2)$ & $430(1)$ & $64(1)$ \\
\hline$C(15)$ & $13450(3)$ & $2362(2)$ & $-357(1)$ & $57(1)$ \\
\hline$C(16)$ & $13677(3)$ & $3892(2)$ & $-997(1)$ & $68(1)$ \\
\hline$C\left(15^{\prime}\right)$ & $12220(40)$ & $3110(30)$ & $-584(16)$ & $97(6)$ \\
\hline$C\left(16^{\prime}\right)$ & $14660(50)$ & $2980(40)$ & $-610(20)$ & $106(7)$ \\
\hline$C(17)$ & $15565(4)$ & $3821(3)$ & $-1702(2)$ & $94(1)$ \\
\hline $\mathrm{C}(18)$ & $12825(2)$ & $5943(2)$ & $4132(1)$ & $53(1)$ \\
\hline
\end{tabular}


Table 3. Bond lengths $[\AA]$ and angles $\left[^{\circ}\right]$ for endo,exo-6-(4-Cyanophenyl)-3-npentylbicyclo[3.1.0]hexan 155b.

\begin{tabular}{|l|l|l|l|l|c|}
\hline $\mathrm{N}(1)-\mathrm{C}(18)$ & $1.1446(17)$ & $\mathrm{C}(5)-\mathrm{C}(6)$ & $1.499(2)$ & $\mathrm{C}(11)-\mathrm{C}(12)$ & $1.3736(18)$ \\
\hline $\mathrm{C}(1)-\mathrm{C}(6)$ & $1.501(2)$ & $\mathrm{C}(6)-\mathrm{C}(7)$ & $1.4845(17)$ & $\mathrm{C}(13)-\mathrm{C}(14)$ & $1.506(2)$ \\
\hline $\mathrm{C}(1)-\mathrm{C}(5)$ & $1.502(2)$ & $\mathrm{C}(7)-\mathrm{C}(8)$ & $1.3889(17)$ & $\mathrm{C}(14)-\mathrm{C}(15)$ & $1.519(2)$ \\
\hline $\mathrm{C}(1)-\mathrm{C}(2)$ & $1.5032(19)$ & $\mathrm{C}(7)-\mathrm{C}(12)$ & $1.3900(17)$ & $\mathrm{C}(14)-\mathrm{C}\left(15^{\prime}\right)$ & $1.56(2)$ \\
\hline $\mathrm{C}(2)-\mathrm{C}(3)$ & $1.5250(18)$ & $\mathrm{C}(8)-\mathrm{C}(9)$ & $1.3783(18)$ & $\mathrm{C}(15)-\mathrm{C}(16)$ & $1.513(3)$ \\
\hline $\mathrm{C}(3)-\mathrm{C}(13)$ & $1.5173(19)$ & $\mathrm{C}(9)-\mathrm{C}(10)$ & $1.3863(18)$ & $\mathrm{C}(16)-\mathrm{C}(17)$ & $1.503(3)$ \\
\hline $\mathrm{C}(3)-\mathrm{C}(4)$ & $1.5380(18)$ & $\mathrm{C}(10)-\mathrm{C}(11)$ & $1.3898(18)$ & $\mathrm{C}\left(15^{\prime}\right)-\mathrm{C}\left(16^{\prime}\right)$ & $1.48(4)$ \\
\hline $\mathrm{C}(4)-\mathrm{C}(5)$ & $1.508(2)$ & $\mathrm{C}(10)-\mathrm{C}(18)$ & $1.4387(18)$ & $\mathrm{C}\left(16^{\prime}\right)-\mathrm{C}(17)$ & $1.75(3)$ \\
\hline
\end{tabular}

\begin{tabular}{|l|c|l|c|}
\hline $\mathrm{C}(6)-\mathrm{C}(1)-\mathrm{C}(5)$ & $59.92(9)$ & $\mathrm{C}(9)-\mathrm{C}(8)-\mathrm{C}(7)$ & $121.11(12)$ \\
\hline $\mathrm{C}(6)-\mathrm{C}(1)-\mathrm{C}(2)$ & $119.94(11)$ & $\mathrm{C}(8)-\mathrm{C}(9)-\mathrm{C}(10)$ & $119.61(12)$ \\
\hline $\mathrm{C}(5)-\mathrm{C}(1)-\mathrm{C}(2)$ & $107.89(12)$ & $\mathrm{C}(9)-\mathrm{C}(10)-\mathrm{C}(11)$ & $120.08(12)$ \\
\hline $\mathrm{C}(1)-\mathrm{C}(2)-\mathrm{C}(3)$ & $106.35(11)$ & $\mathrm{C}(9)-\mathrm{C}(10)-\mathrm{C}(18)$ & $121.11(11)$ \\
\hline $\mathrm{C}(13)-\mathrm{C}(3)-\mathrm{C}(2)$ & $113.23(11)$ & $\mathrm{C}(11)-\mathrm{C}(10)-\mathrm{C}(18)$ & $118.81(12)$ \\
\hline $\mathrm{C}(13)-\mathrm{C}(3)-\mathrm{C}(4)$ & $115.42(12)$ & $\mathrm{C}(12)-\mathrm{C}(11)-\mathrm{C}(10)$ & $119.53(12)$ \\
\hline $\mathrm{C}(2)-\mathrm{C}(3)-\mathrm{C}(4)$ & $104.57(10)$ & $\mathrm{C}(11)-\mathrm{C}(12)-\mathrm{C}(7)$ & $121.30(11)$ \\
\hline $\mathrm{C}(5)-\mathrm{C}(4)-\mathrm{C}(3)$ & $106.08(11)$ & $\mathrm{C}(14)-\mathrm{C}(13)-\mathrm{C}(3)$ & $114.46(12)$ \\
\hline $\mathrm{C}(6)-\mathrm{C}(5)-\mathrm{C}(1)$ & $60.00(10)$ & $\mathrm{C}(13)-\mathrm{C}(14)-\mathrm{C}(15)$ & $112.92(14)$ \\
\hline $\mathrm{C}(6)-\mathrm{C}(5)-\mathrm{C}(4)$ & $118.92(11)$ & $\mathrm{C}(13)-\mathrm{C}(14)-\mathrm{C}\left(15^{\prime}\right)$ & $141.1(9)$ \\
\hline $\mathrm{C}(1)-\mathrm{C}(5)-\mathrm{C}(4)$ & $107.74(11)$ & $\mathrm{C}(15)-\mathrm{C}(14)-\mathrm{C}\left(15^{\prime}\right)$ & $38.3(9)$ \\
\hline $\mathrm{C}(7)-\mathrm{C}(6)-\mathrm{C}(5)$ & $123.48(11)$ & $\mathrm{C}(16)-\mathrm{C}(15)-\mathrm{C}(14)$ & $114.14(15)$ \\
\hline $\mathrm{C}(7)-\mathrm{C}(6)-\mathrm{C}(1)$ & $126.11(10)$ & $\mathrm{C}(17)-\mathrm{C}(16)-\mathrm{C}(15)$ & $112.38(18)$ \\
\hline $\mathrm{C}(5)-\mathrm{C}(6)-\mathrm{C}(1)$ & $60.08(10)$ & $\mathrm{C}\left(16^{\prime}\right)-\mathrm{C}\left(15^{\prime}\right)-\mathrm{C}(14)$ & $97.6(19)$ \\
\hline $\mathrm{C}(8)-\mathrm{C}(7)-\mathrm{C}(12)$ & $118.36(12)$ & $\mathrm{C}\left(15^{\prime}\right)-\mathrm{C}\left(16^{\prime}\right)-\mathrm{C}(17)$ & $105(2)$ \\
\hline $\mathrm{C}(8)-\mathrm{C}(7)-\mathrm{C}(6)$ & $122.03(11)$ & $\mathrm{C}(16)-\mathrm{C}(17)-\mathrm{C}\left(16^{\prime}\right)$ & $38.3(10)$ \\
\hline $\mathrm{C}(12)-\mathrm{C}(7)-\mathrm{C}(6)$ & $119.54(11)$ & $\mathrm{N}(1)-\mathrm{C}(18)-\mathrm{C}(10)$ & $178.42(14)$ \\
\hline
\end{tabular}


Table 4. Anisotropic displacement parameters $\left(\AA^{2} \times 10^{3}\right)$ for endo,exo-6-(4-Cyanophenyl)-3- $n$ pentylbicyclo[3.1.0]hexan 155b. The anisotropic

displacement factor exponent takes the form: $-2 \pi^{2}\left[h^{2} a^{* 2} U^{11}+\ldots+2 h k a^{*} b^{*} U^{12}\right]$

\begin{tabular}{lllllll}
\hline Atom & $\mathrm{U}^{11}$ & $\mathrm{U}^{22}$ & $\mathrm{U}^{33}$ & $\mathrm{U}^{23}$ & $\mathrm{U}^{13}$ & $\mathrm{U}^{12}$ \\
\hline $\mathrm{N}(1)$ & $68(1)$ & $77(1)$ & $61(1)$ & $-8(1)$ & $-4(1)$ & $-21(1)$ \\
$\mathrm{C}(1)$ & $46(1)$ & $41(1)$ & $78(1)$ & $1(1)$ & $8(1)$ & $-8(1)$ \\
$\mathrm{C}(2)$ & $46(1)$ & $44(1)$ & $70(1)$ & $-3(1)$ & $3(1)$ & $-2(1)$ \\
$\mathrm{C}(3)$ & $43(1)$ & $45(1)$ & $53(1)$ & $-7(1)$ & $-2(1)$ & $-7(1)$ \\
$\mathrm{C}(4)$ & $49(1)$ & $54(1)$ & $62(1)$ & $-9(1)$ & $-12(1)$ & $-4(1)$ \\
$\mathrm{C}(5)$ & $33(1)$ & $55(1)$ & $79(1)$ & $-8(1)$ & $-1(1)$ & $-10(1)$ \\
$\mathrm{C}(6)$ & $38(1)$ & $48(1)$ & $63(1)$ & $1(1)$ & $10(1)$ & $-4(1)$ \\
$\mathrm{C}(7)$ & $39(1)$ & $46(1)$ & $42(1)$ & $-1(1)$ & $6(1)$ & $1(1)$ \\
$\mathrm{C}(8)$ & $46(1)$ & $48(1)$ & $47(1)$ & $4(1)$ & $1(1)$ & $6(1)$ \\
$\mathrm{C}(9)$ & $43(1)$ & $61(1)$ & $44(1)$ & $-2(1)$ & $-3(1)$ & $2(1)$ \\
$\mathrm{C}(10)$ & $45(1)$ & $54(1)$ & $37(1)$ & $-7(1)$ & $6(1)$ & $-5(1)$ \\
$\mathrm{C}(11)$ & $53(1)$ & $43(1)$ & $52(1)$ & $-4(1)$ & $-2(1)$ & $4(1)$ \\
$\mathrm{C}(12)$ & $41(1)$ & $49(1)$ & $53(1)$ & $-3(1)$ & $-5(1)$ & $4(1)$ \\
$\mathrm{C}(13)$ & $57(1)$ & $54(1)$ & $54(1)$ & $-13(1)$ & $0(1)$ & $-9(1)$ \\
$\mathrm{C}(14)$ & $76(1)$ & $61(1)$ & $53(1)$ & $-6(1)$ & $5(1)$ & $-3(1)$ \\
$\mathrm{C}(15)$ & $66(1)$ & $55(1)$ & $47(1)$ & $-4(1)$ & $0(1)$ & $2(1)$ \\
$\mathrm{C}(16)$ & $75(1)$ & $69(1)$ & $53(1)$ & $5(1)$ & $8(1)$ & $5(1)$ \\
$\mathrm{C}(17)$ & $92(1)$ & $112(2)$ & $66(1)$ & $11(1)$ & $16(1)$ & $12(1)$ \\
$\mathrm{C}(18)$ & $52(1)$ & $64(1)$ & $41(1)$ & $-7(1)$ & $2(1)$ & $-6(1)$ \\
& & & & & & \\
\hline & & & & & & \\
& & & & & \\
& & & & & \\
\end{tabular}


Table 5. Hydrogen coordinates ( x 104) and isotropic displacement parameters $\left(\AA^{2} \times 10^{3}\right)$ for endo,exo-6-(4-Cyanophenyl)-3-n-pentylbicyclo[3.1.0]hexan 155b.

\begin{tabular}{|c|c|c|c|c|}
\hline Atom & $\mathrm{x}$ & $\mathrm{y}$ & $\mathrm{z}$ & $\mathrm{U}(\mathrm{eq})$ \\
\hline $\mathrm{H}(1)$ & $7420(20)$ & $-1529(17)$ & $3442(10)$ & $69(4)$ \\
\hline $\mathrm{H}(21)$ & $11410(20)$ & $-726(16)$ & 2901(9) & $58(4)$ \\
\hline $\mathrm{H}(22)$ & $10030(20)$ & $-1431(18)$ & $2182(10)$ & $67(4)$ \\
\hline $\mathrm{H}(3)$ & $10810(20)$ & 1831(15) & $2223(8)$ & $48(3)$ \\
\hline $\mathrm{H}(41)$ & $7480(20)$ & 2651(19) & $1760(10)$ & $62(4)$ \\
\hline $\mathrm{H}(42)$ & $7240(20)$ & $1142(17)$ & $1338(10)$ & $65(4)$ \\
\hline $\mathrm{H}(5)$ & $4990(20)$ & $588(16)$ & $2681(9)$ & $57(4)$ \\
\hline $\mathrm{H}(6)$ & $6270(20)$ & $731(15)$ & 4090(9) & $53(3)$ \\
\hline $\mathrm{H}(8)$ & $11220(20)$ & $832(16)$ & $4327(8)$ & $49(3)$ \\
\hline $\mathrm{H}(9)$ & $13430(20)$ & $2848(16)$ & $4574(9)$ & $61(4)$ \\
\hline $\mathrm{H}(11)$ & $9080(20)$ & $6076(17)$ & $3380(9)$ & $62(4)$ \\
\hline $\mathrm{H}(12)$ & $6900(20)$ & $3972(15)$ & $3150(9)$ & $52(3)$ \\
\hline $\mathrm{H}(131)$ & $10880(20)$ & $87(17)$ & $717(9)$ & $64(4)$ \\
\hline $\mathrm{H}(132)$ & $13110(30)$ & $509(18)$ & $1171(10)$ & $74(4)$ \\
\hline $\mathrm{H}(141)$ & $10310(20)$ & $2936(16)$ & 233(9) & $57(4)$ \\
\hline $\mathrm{H}(142)$ & $12010(30)$ & $3360(20)$ & $773(11)$ & $79(5)$ \\
\hline $\mathrm{H}(151)$ & $14970(30)$ & $2050(20)$ & $-134(12)$ & $83(5)$ \\
\hline $\mathrm{H}(152)$ & $13080(30)$ & $1500(20)$ & $-691(13)$ & $100(6)$ \\
\hline $\mathrm{H}(161)$ & $13740(30)$ & $4760(30)$ & $-646(15)$ & $114(7)$ \\
\hline $\mathrm{H}(162)$ & $12180(40)$ & $4150(30)$ & $-1256(15)$ & $121(7)$ \\
\hline $\mathrm{H}(171)$ & $16950(30)$ & $3450(20)$ & $-1418(13)$ & $100(6)$ \\
\hline $\mathrm{H}(172)$ & $15210(40)$ & $2880(30)$ & $-2040(16)$ & $130(9)$ \\
\hline $\mathrm{H}(173)$ & $15680(30)$ & $4870(30)$ & $-2090(15)$ & $117(7)$ \\
\hline
\end{tabular}


Table 6. Torsion angles $\left[{ }^{\circ}\right]$ for endo,exo-6-(4-Cyanophenyl)-3-n-pentylbicyclo[3.1.0]hexan 155b.

\begin{tabular}{|c|c|c|c|}
\hline $\mathrm{C}(6)-\mathrm{C}(1)-\mathrm{C}(2)-\mathrm{C}(3)$ & $-47.57(18)$ & $\mathrm{C}(7)-\mathrm{C}(8)-\mathrm{C}(9)-\mathrm{C}(10)$ & $-0.01(18)$ \\
\hline$C(5)-C(1)-C(2)-C(3)$ & $17.47(16)$ & $\mathrm{C}(8)-\mathrm{C}(9)-\mathrm{C}(10)-\mathrm{C}(11)$ & $0.23(18)$ \\
\hline$C(1)-C(2)-C(3)-C(13)$ & $-153.06(12)$ & $\mathrm{C}(8)-\mathrm{C}(9)-\mathrm{C}(10)-\mathrm{C}(18)$ & $179.82(11)$ \\
\hline$C(1)-C(2)-C(3)-C(4)$ & $-26.60(15)$ & $\mathrm{C}(9)-\mathrm{C}(10)-\mathrm{C}(11)-\mathrm{C}(12)$ & $0.33(18)$ \\
\hline $\mathrm{C}(13)-\mathrm{C}(3)-\mathrm{C}(4)-\mathrm{C}(5)$ & $150.95(12)$ & $\mathrm{C}(18)-\mathrm{C}(10)-\mathrm{C}(11)-\mathrm{C}(12)$ & $-179.27(11)$ \\
\hline $\mathrm{C}(2)-\mathrm{C}(3)-\mathrm{C}(4)-\mathrm{C}(5)$ & $25.87(15)$ & $\mathrm{C}(10)-\mathrm{C}(11)-\mathrm{C}(12)-\mathrm{C}(7)$ & $-1.14(18)$ \\
\hline $\mathrm{C}(2)-\mathrm{C}(1)-\mathrm{C}(5)-\mathrm{C}(6)$ & $-114.78(12)$ & $\mathrm{C}(8)-\mathrm{C}(7)-\mathrm{C}(12)-\mathrm{C}(11)$ & $1.34(18)$ \\
\hline $\mathrm{C}(6)-\mathrm{C}(1)-\mathrm{C}(5)-\mathrm{C}(4)$ & $113.67(12)$ & $\mathrm{C}(6)-\mathrm{C}(7)-\mathrm{C}(12)-\mathrm{C}(11)$ & $178.32(12)$ \\
\hline$C(2)-C(1)-C(5)-C(4)$ & $-1.10(15)$ & $C(2)-C(3)-C(13)-C(14)$ & $-176.68(13)$ \\
\hline $\mathrm{C}(3)-\mathrm{C}(4)-\mathrm{C}(5)-\mathrm{C}(6)$ & $49.45(16)$ & $\mathrm{C}(4)-\mathrm{C}(3)-\mathrm{C}(13)-\mathrm{C}(14)$ & $62.86(16)$ \\
\hline $\mathrm{C}(3)-\mathrm{C}(4)-\mathrm{C}(5)-\mathrm{C}(1)$ & $-15.53(15)$ & $\mathrm{C}(3)-\mathrm{C}(13)-\mathrm{C}(14)-\mathrm{C}(15)$ & $168.61(13)$ \\
\hline $\mathrm{C}(1)-\mathrm{C}(5)-\mathrm{C}(6)-\mathrm{C}(7)$ & $115.77(13)$ & $\mathrm{C}(3)-\mathrm{C}(13)-\mathrm{C}(14)-\mathrm{C}\left(15^{\prime}\right)$ & $-157.7(14)$ \\
\hline$C(4)-C(5)-C(6)-C(7)$ & $21.00(19)$ & $\mathrm{C}(13)-\mathrm{C}(14)-\mathrm{C}(15)-\mathrm{C}(16)$ & $179.36(16)$ \\
\hline $\mathrm{C}(4)-\mathrm{C}(5)-\mathrm{C}(6)-\mathrm{C}(1)$ & $-94.76(14)$ & $\begin{array}{l}C\left(15^{\prime}\right)-C(14)-C(15)- \\
C(16)\end{array}$ & $33.5(13)$ \\
\hline $\mathrm{C}(5)-\mathrm{C}(1)-\mathrm{C}(6)-\mathrm{C}(7)$ & $-111.58(15)$ & $\mathrm{C}(14)-\mathrm{C}(15)-\mathrm{C}(16)-\mathrm{C}(17)$ & $173.37(19)$ \\
\hline $\mathrm{C}(2)-\mathrm{C}(1)-\mathrm{C}(6)-\mathrm{C}(7)$ & $-17.2(2)$ & $\begin{array}{l}C(13)-C(14)-C\left(15^{\prime}\right)- \\
C\left(16^{\prime}\right)\end{array}$ & $-82.4(18)$ \\
\hline $\mathrm{C}(2)-\mathrm{C}(1)-\mathrm{C}(6)-\mathrm{C}(5)$ & $94.36(14)$ & $\begin{array}{l}\mathrm{C}(15)-\mathrm{C}(14)-\mathrm{C}\left(15^{\prime}\right)- \\
\mathrm{C}\left(16^{\prime}\right)\end{array}$ & $-27.0(12)$ \\
\hline$C(5)-C(6)-C(7)-C(8)$ & $-129.32(14)$ & $\begin{array}{l}\mathrm{C}(14)-\mathrm{C}\left(15^{\prime}\right)-\mathrm{C}\left(16^{\prime}\right)- \\
\mathrm{C}(17)\end{array}$ & $-175.9(13)$ \\
\hline $\mathrm{C}(1)-\mathrm{C}(6)-\mathrm{C}(7)-\mathrm{C}(8)$ & $-54.26(18)$ & $\begin{array}{l}\mathrm{C}(15)-\mathrm{C}(16)-\mathrm{C}(17)- \\
\mathrm{C}\left(16^{\prime}\right)\end{array}$ & $-25.4(14)$ \\
\hline$C(5)-C(6)-C(7)-C(12)$ & $53.81(17)$ & $\begin{array}{l}\mathrm{C}\left(15^{\prime}\right)-\mathrm{C}\left(16^{\prime}\right)-\mathrm{C}(17)- \\
\mathrm{C}(16)\end{array}$ & $27.7(12)$ \\
\hline$C(1)-C(6)-C(7)-C(12)$ & $128.87(14)$ & $\mathrm{C}(9)-\mathrm{C}(10)-\mathrm{C}(18)-\mathrm{N}(1)$ & $163(5)$ \\
\hline $\mathrm{C}(12)-\mathrm{C}(7)-\mathrm{C}(8)-\mathrm{C}(9)$ & $-0.76(17)$ & $\mathrm{C}(11)-\mathrm{C}(10)-\mathrm{C}(18)-\mathrm{N}(1)$ & $-18(5)$ \\
\hline$C(6)-C(7)-C(8)-C(9)$ & $-177.66(11)$ & & \\
\hline
\end{tabular}

Table 7. Hydrogen bonds for endo,exo-6-(4-Cyanophenyl)-3-n-pentylbicyclo[3.1.0]hexan 155b [ $\AA$ and ${ }^{\circ}$.

\begin{tabular}{lcccc}
\hline D-H...A & $\mathrm{d}(\mathrm{D}-\mathrm{H})$ & $\mathrm{d}(\mathrm{H} \ldots \mathrm{A})$ & $\mathrm{d}(\mathrm{D} \ldots \mathrm{A})$ & $<(\mathrm{DHA})$ \\
\hline $\mathrm{C}(9)-\mathrm{H}(9) \ldots \mathrm{N}(1) \# 1$ & $0.953(14)$ & $2.563(14)$ & $3.451(2)$ & $155.2(11)$
\end{tabular}


Symmetry transformations used to generate equivalent atoms:

$\# 1-x+3,-y+1,-z+1$ 

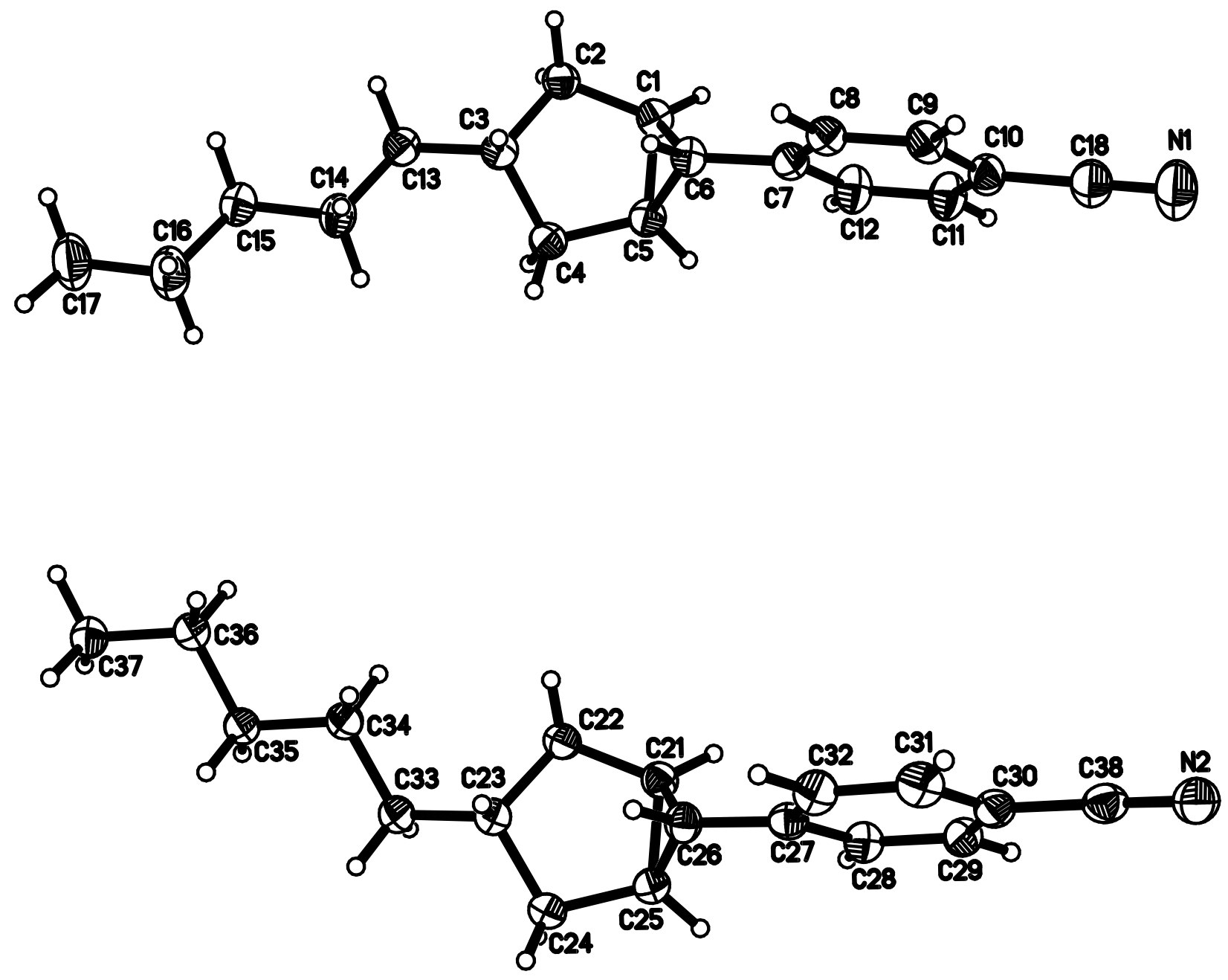

Table 1. Crystal data and structure refinement for exo,exo-6-(4-Cyanophenyl)-3-n-pentylbicyclo[3.1.0]hexane

\section{$155 a$.}

Identification code: exo,exo-6-(4-Cyanophenyl)-3-n-pentylbicyclo[3.1.0]hexane 155a

Empirical formula

Formula weight

Temperature

Wavelength

Crystal system

Space group

Unit cell dimensions

Volume

Z

Density (calculated)

Absorption coefficient
$\mathrm{C}_{18} \mathrm{H}_{23} \mathrm{~N}$

253.37

120(2) K

$0.71073 \AA$

Triclinic

P -1

$\mathrm{a}=9.7909(4) \AA$

$\alpha=104.734(1)^{\circ}$.

$\mathrm{b}=11.8250(5) \AA$

$\beta=103.126(1)^{\circ}$.

$\mathrm{c}=14.2577(6) \AA$

$\gamma=97.423(1)^{\circ}$.
4

$1.104 \mathrm{Mg} / \mathrm{m}^{3}$

$0.063 \mathrm{~mm}^{-1}$ 
$\mathrm{F}(000)$

Crystal size

Theta range for data collection Index ranges

Reflections collected

Independent reflections

Completeness to theta $=30.00^{\circ}$

Absorption correction

Max. and min. transmission

Refinement method

Data / restraints / parameters

Goodness-of-fit on $\mathrm{F}^{2}$

Final $\mathrm{R}$ indices [I $>2 \operatorname{sigma}(\mathrm{I})]$

$\mathrm{R}$ indices (all data)

Largest diff. peak and hole
552

$0.40 \times 0.24 \times 0.10 \mathrm{~mm}^{3}$

1.53 to $30.00^{\circ}$.

$-13<=\mathrm{h}<=13,-13<=\mathrm{k}<=16,-20<=\mathrm{l}<=18$

13531

$8684[\mathrm{R}($ int $)=0.0309]$

$97.7 \%$

None

0.9937 and 0.9751

Full-matrix least-squares on $\mathrm{F}^{2}$

8684 / 0 / 527

0.935

$\mathrm{R} 1=0.0489, \mathrm{wR} 2=0.1171$

$\mathrm{R} 1=0.0783, \mathrm{wR} 2=0.1300$

0.313 and -0.190 e. $\AA^{-3}$ 
6. exo,exo-Bicyclo[3.1.0]hexan-3,6-dicarbonsäure-3,6-di(4-n-pentylphenyl)ester 35a

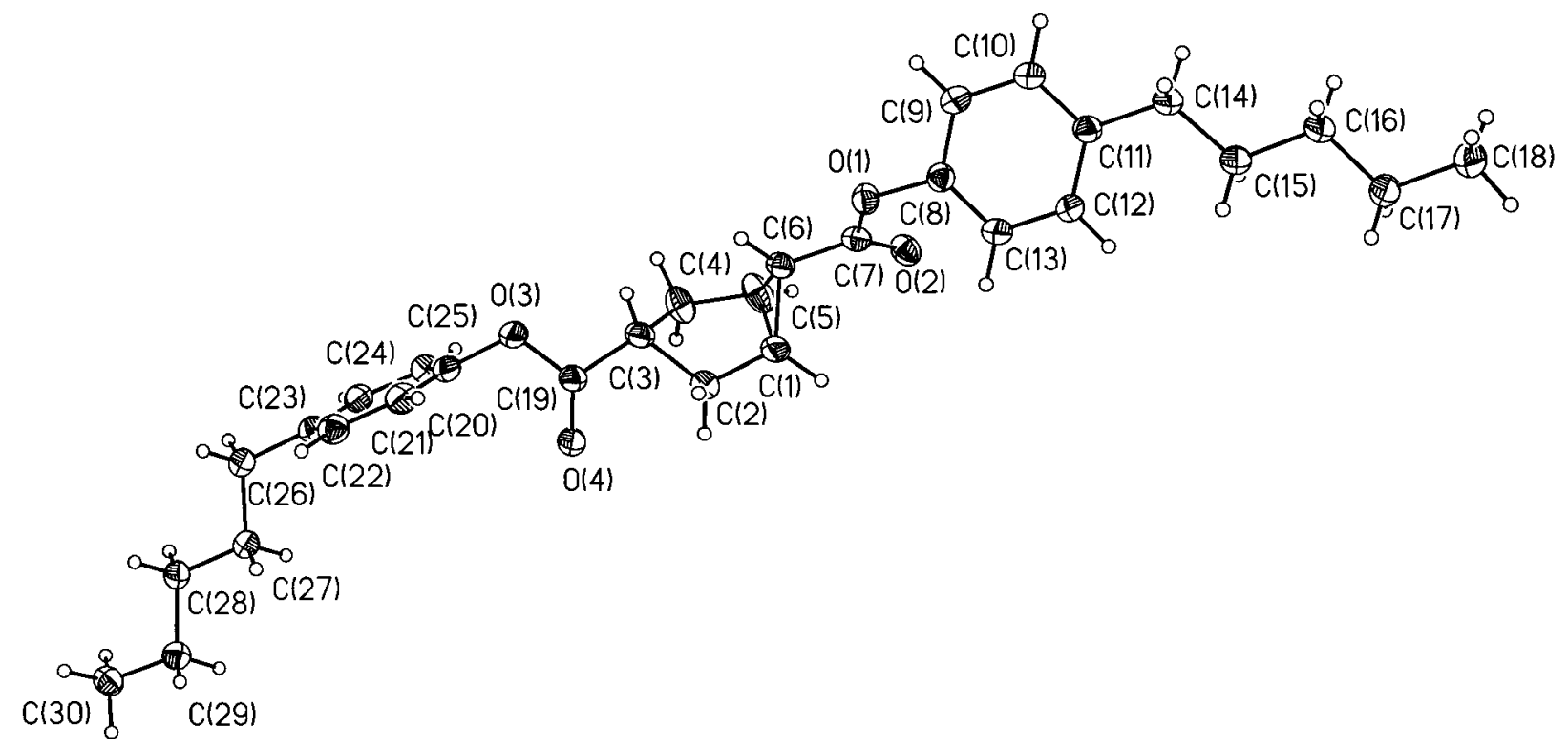

Identification code

exo,exo-Bicyclo[3.1.0]hexan-3,6-dicarbonsäure-3,6-di(4- $n$-pentylphenyl)ester 35a

$\begin{array}{lc}\text { Empirical formula } & \text { C30 H38 O6 } \\ \text { Formula weight } & 494.60 \\ \text { Temperature } & 133(2) \mathrm{K} \\ \text { Wavelength } & 0.71073 \mathrm{~A}\end{array}$

Crystal system, space group monoclinic, C 2/c

Unit cell dimensions $\quad \mathrm{a}=28.7930(13) \mathrm{A} \quad$ alpha $=90 \mathrm{deg}$.

$\mathrm{b}=5.9710(5) \mathrm{A} \quad$ beta $=111.767(7) \mathrm{deg}$.

Volume $\quad 5176.8(5) \mathrm{A}^{\wedge} 3$

$\mathrm{Z}$, Calculated density $\quad 8,1.269 \mathrm{Mg} / \mathrm{m}^{\wedge} 3$

Absorption coefficient $\quad 0.087 \mathrm{~mm}^{\wedge}-1$

$\mathrm{F}(000) \quad 2128$

Theta range for data collection 1.35 to $24.06 \mathrm{deg}$.

Index ranges $\quad-32<=\mathrm{h}<=32,-6<=\mathrm{k}<=6,-37<=\mathrm{l}<=37$

Reflections collected / unique $28013 / 4058$ [R(int) $=0.0696]$

Observed reflections $\quad 3028$

[I $>2 \operatorname{sigma}(\mathrm{I})]$

Completeness to theta $=24.06 \quad 99.5 \%$

Refinement method $\quad$ Full-matrix least-squares on $\mathrm{F}^{\wedge} 2$

Data / restraints / parameters 4058 / 0/309

Goodness-of-fit on $\mathrm{F}^{\wedge} 2 \quad 1.053$

Final R indices $[\mathrm{I}>2 \operatorname{sigma}(\mathrm{I})] \quad \mathrm{R} 1=0.0506, \mathrm{wR} 2=0.1342$

$\mathrm{R}$ indices (all data) $\mathrm{R} 1=0.0690, \mathrm{wR} 2=0.1421$

Largest diff. peak and hole $\quad 0.587$ and -0.373 e. $\mathrm{A}^{\wedge}-3$ 
Table 2. Atomic coordinates $\left(\times 10^{4}\right)$ and equivalent isotropic displacement parameters $\left(\AA^{2} \times 10^{3}\right)$ for exo,exoBicyclo[3.1.0]hexan-3,6-dicarbonsäure-3,6-di(4-n-pentylphenyl)ester 35a. U(eq) is defined as one third of the trace of the orthogonalized $\mathrm{U}^{\mathrm{ij}}$ tensor.

\begin{tabular}{|c|c|c|c|c|}
\hline Atom & $\mathrm{x}$ & $\mathrm{y}$ & $\mathrm{z}$ & $\mathrm{U}(\mathrm{eq})$ \\
\hline $\mathrm{N}(1)$ & $7656(1)$ & $-3652(1)$ & $3409(1)$ & $54(1)$ \\
\hline $\mathrm{C}(1)$ & $4547(1)$ & $1162(1)$ & 1159(1) & $29(1)$ \\
\hline $\mathrm{C}(2)$ & $4114(1)$ & $2357(1)$ & $1222(1)$ & $31(1)$ \\
\hline $\mathrm{C}(3)$ & $2538(1)$ & $2162(1)$ & $1247(1)$ & $28(1)$ \\
\hline $\mathrm{C}(4)$ & $1938(1)$ & $847(1)$ & $627(1)$ & $30(1)$ \\
\hline$C(5)$ & $3194(1)$ & $225(1)$ & $788(1)$ & $30(1)$ \\
\hline$C(6)$ & $4113(1)$ & $496(1)$ & 1864(1) & $28(1)$ \\
\hline$C(7)$ & $4926(1)$ & $-386(1)$ & 2171(1) & $28(1)$ \\
\hline $\mathrm{C}(8)$ & $5236(1)$ & $-388(1)$ & $3176(1)$ & $29(1)$ \\
\hline $\mathrm{C}(9)$ & 5949(1) & $-1220(1)$ & $3496(1)$ & $32(1)$ \\
\hline$C(10)$ & $6367(1)$ & $-2072(1)$ & $2806(1)$ & $33(1)$ \\
\hline $\mathrm{C}(11)$ & $6104(2)$ & $-2067(1)$ & $1805(1)$ & $39(1)$ \\
\hline$C(12)$ & $5395(2)$ & $-1227(1)$ & 1498(1) & $37(1)$ \\
\hline$C(13)$ & $1707(1)$ & $3054(1)$ & $898(1)$ & $31(1)$ \\
\hline$C(14)$ & $167(1)$ & $2878(1)$ & $964(1)$ & $33(1)$ \\
\hline$C(15)$ & $-614(1)$ & $3849(1)$ & $734(1)$ & $34(1)$ \\
\hline$C(16)$ & $-2138(2)$ & $3691(2)$ & $818(2)$ & $53(1)$ \\
\hline$C(17)$ & $-2912(2)$ & $4652(2)$ & $577(2)$ & $59(1)$ \\
\hline$C(18)$ & $7084(1)$ & $-2957(1)$ & $3137(1)$ & $39(1)$ \\
\hline $\mathrm{N}(2)$ & 4049(1) & $2154(1)$ & $4442(1)$ & $39(1)$ \\
\hline $\mathrm{C}(21)$ & $2349(1)$ & $8074(1)$ & $3612(1)$ & $27(1)$ \\
\hline$C(22)$ & $1628(1)$ & $9134(1)$ & $3788(1)$ & $29(1)$ \\
\hline$C(23)$ & $408(1)$ & $8892(1)$ & $2824(1)$ & $30(1)$ \\
\hline $\mathrm{C}(24)$ & $957(2)$ & $8141(1)$ & 1986(1) & $34(1)$ \\
\hline $\mathrm{C}(25)$ & $1946(1)$ & $7460(1)$ & 2498(1) & $30(1)$ \\
\hline$C(26)$ & 1391(1) & $6835(1)$ & $3187(1)$ & $28(1)$ \\
\hline $\mathrm{C}(27)$ & $1987(1)$ & $5825(1)$ & $3431(1)$ & $27(1)$ \\
\hline $\mathrm{C}(28)$ & $3388(1)$ & $5691(1)$ & $3428(1)$ & $29(1)$ \\
\hline$C(29)$ & $3939(1)$ & $4763(1)$ & 3693(1) & $29(1)$ \\
\hline$C(30)$ & $3086(1)$ & $3944(1)$ & $3979(1)$ & $29(1)$ \\
\hline$C(31)$ & $1693(1)$ & $4064(1)$ & 3993(1) & $33(1)$ \\
\hline$C(32)$ & $1150(1)$ & 4989(1) & $3713(1)$ & $32(1)$ \\
\hline $\mathrm{C}(33)$ & $-81(1)$ & $9998(1)$ & 2623(1) & 31(1) \\
\hline$C(34)$ & $-610(1)$ & $10727(1)$ & $3464(1)$ & $30(1)$ \\
\hline$C(35)$ & $-1445(1)$ & 11641(1) & $3166(1)$ & $28(1)$ \\
\hline$C(36)$ & $-1919(1)$ & $12388(1)$ & 4029(1) & $30(1)$ \\
\hline$C(37)$ & $-2814(1)$ & $13257(1)$ & $3724(1)$ & $35(1)$ \\
\hline $\mathrm{C}(38)$ & $3633(1)$ & 2958(1) & $4246(1)$ & $32(1)$ \\
\hline
\end{tabular}


Table 3. Bond lengths $[\AA]$ and angles $\left[{ }^{\circ}\right]$ for exo,exo-Bicyclo[3.1.0]hexan-3,6-dicarbonsäure-3,6-di(4-npentylphenyl)ester 35a.

\begin{tabular}{|l|l|l|l|l|l|}
\hline $\mathrm{N}(1)-\mathrm{C}(18)$ & $1.150(2)$ & $\mathrm{C}(10)-\mathrm{C}(11)$ & $1.394(2)$ & $\mathrm{C}(24)-\mathrm{C}(25)$ & $1.517(2)$ \\
\hline $\mathrm{C}(1)-\mathrm{C}(5)$ & $1.505(2)$ & $\mathrm{C}(10)-\mathrm{C}(18)$ & $1.450(2)$ & $\mathrm{C}(25)-\mathrm{C}(26)$ & $1.522(2)$ \\
\hline $\mathrm{C}(1)-\mathrm{C}(2)$ & $1.513(2)$ & $\mathrm{C}(11)-\mathrm{C}(12)$ & $1.386(2)$ & $\mathrm{C}(26)-\mathrm{C}(27)$ & $1.478(2)$ \\
\hline $\mathrm{C}(1)-\mathrm{C}(6)$ & $1.524(2)$ & $\mathrm{C}(13)-\mathrm{C}(14)$ & $1.524(2)$ & $\mathrm{C}(27)-\mathrm{C}(32)$ & $1.398(2)$ \\
\hline $\mathrm{C}(2)-\mathrm{C}(3)$ & $1.540(2)$ & $\mathrm{C}(14)-\mathrm{C}(15)$ & $1.522(2)$ & $\mathrm{C}(27)-\mathrm{C}(28)$ & $1.402(2)$ \\
\hline $\mathrm{C}(3)-\mathrm{C}(13)$ & $1.525(2)$ & $\mathrm{C}(15)-\mathrm{C}(16)$ & $1.516(2)$ & $\mathrm{C}(28)-\mathrm{C}(29)$ & $1.382(2)$ \\
\hline $\mathrm{C}(3)-\mathrm{C}(4)$ & $1.540(2)$ & $\mathrm{C}(16)-\mathrm{C}(17)$ & $1.512(2)$ & $\mathrm{C}(29)-\mathrm{C}(30)$ & $1.396(2)$ \\
\hline $\mathrm{C}(4)-\mathrm{C}(5)$ & $1.515(2)$ & $\mathrm{N}(2)-\mathrm{C}(38)$ & $1.154(2)$ & $\mathrm{C}(30)-\mathrm{C}(31)$ & $1.393(2)$ \\
\hline $\mathrm{C}(5)-\mathrm{C}(6)$ & $1.521(2)$ & $\mathrm{C}(21)-\mathrm{C}(25)$ & $1.504(2)$ & $\mathrm{C}(30)-\mathrm{C}(38)$ & $1.444(2)$ \\
\hline $\mathrm{C}(6)-\mathrm{C}(7)$ & $1.484(2)$ & $\mathrm{C}(21)-\mathrm{C}(22)$ & $1.510(2)$ & $\mathrm{C}(31)-\mathrm{C}(32)$ & $1.384(2)$ \\
\hline $\mathrm{C}(7)-\mathrm{C}(12)$ & $1.396(2)$ & $\mathrm{C}(21)-\mathrm{C}(26)$ & $1.527(2)$ & $\mathrm{C}(33)-\mathrm{C}(34)$ & $1.523(2)$ \\
\hline $\mathrm{C}(7)-\mathrm{C}(8)$ & $1.397(2)$ & $\mathrm{C}(22)-\mathrm{C}(23)$ & $1.537(2)$ & $\mathrm{C}(34)-\mathrm{C}(35)$ & $1.523(2)$ \\
\hline $\mathrm{C}(8)-\mathrm{C}(9)$ & $1.387(2)$ & $\mathrm{C}(23)-\mathrm{C}(33)$ & $1.521(2)$ & $\mathrm{C}(35)-\mathrm{C}(36)$ & $1.523(2)$ \\
\hline $\mathrm{C}(9)-\mathrm{C}(10)$ & $1.391(2)$ & $\mathrm{C}(23)-\mathrm{C}(24)$ & $1.544(2)$ & $\mathrm{C}(36)-\mathrm{C}(37)$ & $1.517(2)$ \\
\hline
\end{tabular}

\begin{tabular}{|l|c|l|c|}
\hline $\mathrm{C}(5)-\mathrm{C}(1)-\mathrm{C}(2)$ & $107.33(10)$ & $\mathrm{C}(25)-\mathrm{C}(21)-\mathrm{C}(22)$ & $108.29(10)$ \\
\hline $\mathrm{C}(5)-\mathrm{C}(1)-\mathrm{C}(6)$ & $60.30(8)$ & $\mathrm{C}(25)-\mathrm{C}(21)-\mathrm{C}(26)$ & $60.31(8)$ \\
\hline $\mathrm{C}(2)-\mathrm{C}(1)-\mathrm{C}(6)$ & $116.96(10)$ & $\mathrm{C}(22)-\mathrm{C}(21)-\mathrm{C}(26)$ & $117.54(10)$ \\
\hline $\mathrm{C}(1)-\mathrm{C}(2)-\mathrm{C}(3)$ & $105.63(10)$ & $\mathrm{C}(21)-\mathrm{C}(22)-\mathrm{C}(23)$ & $105.28(10)$ \\
\hline $\mathrm{C}(13)-\mathrm{C}(3)-\mathrm{C}(2)$ & $114.49(10)$ & $\mathrm{C}(33)-\mathrm{C}(23)-\mathrm{C}(22)$ & $114.93(10)$ \\
\hline $\mathrm{C}(13)-\mathrm{C}(3)-\mathrm{C}(4)$ & $115.09(9)$ & $\mathrm{C}(33)-\mathrm{C}(23)-\mathrm{C}(24)$ & $114.77(10)$ \\
\hline $\mathrm{C}(2)-\mathrm{C}(3)-\mathrm{C}(4)$ & $103.74(10)$ & $\mathrm{C}(22)-\mathrm{C}(23)-\mathrm{C}(24)$ & $104.22(10)$ \\
\hline $\mathrm{C}(5)-\mathrm{C}(4)-\mathrm{C}(3)$ & $105.21(9)$ & $\mathrm{C}(25)-\mathrm{C}(24)-\mathrm{C}(23)$ & $105.46(10)$ \\
\hline $\mathrm{C}(1)-\mathrm{C}(5)-\mathrm{C}(4)$ & $107.97(10)$ & $\mathrm{C}(21)-\mathrm{C}(25)-\mathrm{C}(24)$ & $107.40(10)$ \\
\hline $\mathrm{C}(1)-\mathrm{C}(5)-\mathrm{C}(6)$ & $60.45(8)$ & $\mathrm{C}(21)-\mathrm{C}(25)-\mathrm{C}(26)$ & $60.58(8)$ \\
\hline $\mathrm{C}(4)-\mathrm{C}(5)-\mathrm{C}(6)$ & $117.09(10)$ & $\mathrm{C}(24)-\mathrm{C}(25)-\mathrm{C}(26)$ & $116.63(10)$ \\
\hline $\mathrm{C}(7)-\mathrm{C}(6)-\mathrm{C}(5)$ & $120.55(10)$ & $\mathrm{C}(27)-\mathrm{C}(26)-\mathrm{C}(25)$ & $120.85(10)$ \\
\hline $\mathrm{C}(7)-\mathrm{C}(6)-\mathrm{C}(1)$ & $120.92(10)$ & $\mathrm{C}(27)-\mathrm{C}(26)-\mathrm{C}(21)$ & $117.95(9)$ \\
\hline $\mathrm{C}(5)-\mathrm{C}(6)-\mathrm{C}(1)$ & $59.25(8)$ & $\mathrm{C}(25)-\mathrm{C}(26)-\mathrm{C}(21)$ & $59.10(8)$ \\
\hline $\mathrm{C}(12)-\mathrm{C}(7)-\mathrm{C}(8)$ & $117.99(11)$ & $\mathrm{C}(32)-\mathrm{C}(27)-\mathrm{C}(28)$ & $118.03(11)$ \\
\hline $\mathrm{C}(12)-\mathrm{C}(7)-\mathrm{C}(6)$ & $123.09(11)$ & $\mathrm{C}(32)-\mathrm{C}(27)-\mathrm{C}(26)$ & $119.28(10)$ \\
\hline $\mathrm{C}(8)-\mathrm{C}(7)-\mathrm{C}(6)$ & $118.92(11)$ & $\mathrm{C}(28)-\mathrm{C}(27)-\mathrm{C}(26)$ & $122.64(10)$ \\
\hline $\mathrm{C}(9)-\mathrm{C}(8)-\mathrm{C}(7)$ & $121.29(12)$ & $\mathrm{C}(29)-\mathrm{C}(28)-\mathrm{C}(27)$ & $121.61(11)$ \\
\hline $\mathrm{C}(8)-\mathrm{C}(9)-\mathrm{C}(10)$ & $119.67(12)$ & $\mathrm{C}(28)-\mathrm{C}(29)-\mathrm{C}(30)$ & $119.30(11)$ \\
\hline $\mathrm{C}(9)-\mathrm{C}(10)-\mathrm{C}(11)$ & $120.01(11)$ & $\mathrm{C}(31)-\mathrm{C}(30)-\mathrm{C}(29)$ & $120.06(11)$ \\
\hline $\mathrm{C}(9)-\mathrm{C}(10)-\mathrm{C}(18)$ & $119.63(12)$ & $\mathrm{C}(31)-\mathrm{C}(30)-\mathrm{C}(38)$ & $119.72(11)$ \\
\hline $\mathrm{C}(11)-\mathrm{C}(10)-\mathrm{C}(18)$ & $120.36(12)$ & $\mathrm{C}(29)-\mathrm{C}(30)-\mathrm{C}(38)$ & $120.21(11)$ \\
\hline $\mathrm{C}(12)-\mathrm{C}(11)-\mathrm{C}(10)$ & $119.56(12)$ & $\mathrm{C}(32)-\mathrm{C}(31)-\mathrm{C}(30)$ & $119.98(11)$ \\
\hline $\mathrm{C}(11)-\mathrm{C}(12)-\mathrm{C}(7)$ & $121.42(12)$ & $\mathrm{C}(31)-\mathrm{C}(32)-\mathrm{C}(27)$ & $121.01(11)$ \\
\hline $\mathrm{C}(14)-\mathrm{C}(13)-\mathrm{C}(3)$ & $113.85(10)$ & $\mathrm{C}(23)-\mathrm{C}(33)-\mathrm{C}(34)$ & $113.71(10)$ \\
\hline $\mathrm{C}(15)-\mathrm{C}(14)-\mathrm{C}(13)$ & $114.07(11)$ & $\mathrm{C}(35)-\mathrm{C}(34)-\mathrm{C}(33)$ & $114.00(10)$ \\
\hline $\mathrm{C}(16)-\mathrm{C}(15)-\mathrm{C}(14)$ & $114.27(11)$ & $\mathrm{C}(34)-\mathrm{C}(35)-\mathrm{C}(36)$ & $113.10(10)$ \\
\hline $\mathrm{C}(17)-\mathrm{C}(16)-\mathrm{C}(15)$ & $113.93(13)$ & $\mathrm{C}(37)-\mathrm{C}(36)-\mathrm{C}(35)$ & $113.40(10)$ \\
\hline $\mathrm{N}(1)-\mathrm{C}(18)-\mathrm{C}(10)$ & $179.35(16)$ & $\mathrm{N}(2)-\mathrm{C}(38)-\mathrm{C}(30)$ & $178.52(13)$ \\
\hline
\end{tabular}


Table 4. Anisotropic displacement parameters $\left(\AA^{2} \times 10^{3}\right)$ for exo,exo-Bicyclo[3.1.0]hexan-3,6-dicarbonsäure3,6-di(4- $n$-pentylphenyl)ester 35a. The anisotropic displacement factor exponent takes the form: $-2 \pi^{2}\left[h^{2}\right.$ $\left.\mathrm{a}^{* 2} \mathrm{U}^{11}+\ldots+2 \mathrm{~h} \mathrm{k} \mathrm{a} \mathrm{b}^{*} \mathrm{U}^{12}\right]$

\begin{tabular}{|c|c|c|c|c|c|c|}
\hline Atom & $\mathrm{U}^{11}$ & $\mathrm{U}^{22}$ & $\mathrm{U}^{33}$ & $\mathrm{U}^{23}$ & $\mathrm{U}^{13}$ & $\mathrm{U}^{12}$ \\
\hline $\mathrm{N}(1)$ & $51(1)$ & $57(1)$ & $74(1)$ & $39(1)$ & $25(1)$ & $25(1)$ \\
\hline$C(1)$ & $30(1)$ & $34(1)$ & $27(1)$ & $12(1)$ & $9(1)$ & $8(1)$ \\
\hline$C(2)$ & $31(1)$ & $30(1)$ & $30(1)$ & 11(1) & $5(1)$ & $4(1)$ \\
\hline$C(3)$ & $33(1)$ & $30(1)$ & $21(1)$ & $7(1)$ & $5(1)$ & $7(1)$ \\
\hline$C(4)$ & $30(1)$ & $31(1)$ & $25(1)$ & $8(1)$ & $4(1)$ & $5(1)$ \\
\hline$C(5)$ & $35(1)$ & $30(1)$ & $24(1)$ & $6(1)$ & $6(1)$ & $8(1)$ \\
\hline$C(6)$ & $32(1)$ & $29(1)$ & $23(1)$ & $8(1)$ & $8(1)$ & $10(1)$ \\
\hline$C(7)$ & $28(1)$ & $29(1)$ & $29(1)$ & $10(1)$ & $8(1)$ & $5(1)$ \\
\hline $\mathrm{C}(8)$ & $27(1)$ & $31(1)$ & $29(1)$ & 11(1) & $9(1)$ & $5(1)$ \\
\hline C(9) & $28(1)$ & $38(1)$ & $34(1)$ & $18(1)$ & $9(1)$ & $6(1)$ \\
\hline$C(10)$ & $28(1)$ & $33(1)$ & $43(1)$ & $19(1)$ & $12(1)$ & $8(1)$ \\
\hline $\mathrm{C}(11)$ & $47(1)$ & $38(1)$ & $37(1)$ & 11(1) & $15(1)$ & $18(1)$ \\
\hline$C(12)$ & $47(1)$ & $41(1)$ & $29(1)$ & $13(1)$ & $13(1)$ & $19(1)$ \\
\hline$C(13)$ & $35(1)$ & $30(1)$ & $26(1)$ & $10(1)$ & $5(1)$ & $8(1)$ \\
\hline $\mathrm{C}(14)$ & $38(1)$ & $34(1)$ & $33(1)$ & $14(1)$ & $11(1)$ & $13(1)$ \\
\hline$C(15)$ & $40(1)$ & $33(1)$ & $33(1)$ & 11(1) & $10(1)$ & $13(1)$ \\
\hline$C(16)$ & $47(1)$ & $49(1)$ & $81(1)$ & $35(1)$ & $27(1)$ & $23(1)$ \\
\hline$C(17)$ & $49(1)$ & $55(1)$ & $92(2)$ & $37(1)$ & $30(1)$ & $28(1)$ \\
\hline $\mathrm{C}(18)$ & $35(1)$ & $42(1)$ & $51(1)$ & $23(1)$ & $18(1)$ & 11(1) \\
\hline $\mathrm{N}(2)$ & $53(1)$ & $31(1)$ & $36(1)$ & 11(1) & $14(1)$ & $9(1)$ \\
\hline $\mathrm{C}(21)$ & $26(1)$ & $28(1)$ & $26(1)$ & $8(1)$ & $5(1)$ & $5(1)$ \\
\hline$C(22)$ & $33(1)$ & $29(1)$ & $26(1)$ & $8(1)$ & $7(1)$ & $7(1)$ \\
\hline $\mathrm{C}(23)$ & $31(1)$ & $33(1)$ & $27(1)$ & $10(1)$ & $9(1)$ & $10(1)$ \\
\hline $\mathrm{C}(24)$ & $41(1)$ & $38(1)$ & $25(1)$ & $10(1)$ & $8(1)$ & $15(1)$ \\
\hline$C(25)$ & $33(1)$ & $34(1)$ & $26(1)$ & $10(1)$ & $11(1)$ & $12(1)$ \\
\hline$C(26)$ & $23(1)$ & $28(1)$ & $31(1)$ & $9(1)$ & $6(1)$ & $5(1)$ \\
\hline$C(27)$ & $27(1)$ & $26(1)$ & $24(1)$ & $4(1)$ & $4(1)$ & $3(1)$ \\
\hline $\mathrm{C}(28)$ & $29(1)$ & $28(1)$ & $32(1)$ & $10(1)$ & $9(1)$ & $4(1)$ \\
\hline $\mathrm{C}(29)$ & $30(1)$ & $29(1)$ & $29(1)$ & $6(1)$ & $7(1)$ & $7(1)$ \\
\hline$C(30)$ & $36(1)$ & $23(1)$ & $25(1)$ & $4(1)$ & $4(1)$ & $4(1)$ \\
\hline$C(31)$ & $34(1)$ & $26(1)$ & $34(1)$ & $7(1)$ & $7(1)$ & $-3(1)$ \\
\hline $\mathrm{C}(32)$ & $27(1)$ & $29(1)$ & $35(1)$ & $6(1)$ & $5(1)$ & $2(1)$ \\
\hline $\mathrm{C}(33)$ & $35(1)$ & $35(1)$ & $28(1)$ & $13(1)$ & $10(1)$ & $14(1)$ \\
\hline $\mathrm{C}(34)$ & $33(1)$ & $32(1)$ & $28(1)$ & $12(1)$ & $10(1)$ & $10(1)$ \\
\hline$C(35)$ & $27(1)$ & $30(1)$ & $27(1)$ & $9(1)$ & $7(1)$ & $7(1)$ \\
\hline$C(36)$ & $29(1)$ & $33(1)$ & $28(1)$ & $10(1)$ & $7(1)$ & $8(1)$ \\
\hline $\mathrm{C}(37)$ & $35(1)$ & $36(1)$ & $35(1)$ & $8(1)$ & $6(1)$ & $12(1)$ \\
\hline $\mathrm{C}(38)$ & $39(1)$ & $27(1)$ & $27(1)$ & $5(1)$ & $9(1)$ & $3(1)$ \\
\hline
\end{tabular}


Table 5. Hydrogen coordinates $\left(\times 10^{4}\right)$ and isotropic displacement parameters $\left(\AA^{2} \times 10^{3}\right)$ for exo,exoBicyclo[3.1.0]hexan-3,6-dicarbonsäure-3,6-di(4-n-pentylphenyl)ester 35a.

\begin{tabular}{|c|c|c|c|c|}
\hline Atom & $\mathrm{x}$ & $\mathrm{y}$ & $\mathrm{z}$ & $\mathrm{U}(\mathrm{eq})$ \\
\hline $\mathrm{H}(1)$ & $5411(15)$ & $995(11)$ & $954(10)$ & $35(4)$ \\
\hline $\mathrm{H}(21)$ & $4196(14)$ & $2589(11)$ & $592(10)$ & $35(3)$ \\
\hline $\mathrm{H}(22)$ & $4720(14)$ & $3016(12)$ & $1804(10)$ & $37(4)$ \\
\hline $\mathrm{H}(31)$ & $2510(13)$ & $2255(11)$ & $1966(10)$ & $30(3)$ \\
\hline $\mathrm{H}(41)$ & $1589(14)$ & $782(12)$ & $-110(10)$ & $38(4)$ \\
\hline $\mathrm{H}(42)$ & $1103(15)$ & $480(12)$ & $818(10)$ & $37(4)$ \\
\hline $\mathrm{H}(5)$ & $3140(14)$ & $-570(12)$ & $336(10)$ & $34(3)$ \\
\hline $\mathrm{H}(6)$ & $3761(13)$ & $943(11)$ & $2381(10)$ & $26(3)$ \\
\hline $\mathrm{H}(8)$ & 4937(13) & 201(11) & $3654(10)$ & $30(3)$ \\
\hline $\mathrm{H}(9)$ & $6135(15)$ & $-1226(12)$ & $4198(11)$ & $39(4)$ \\
\hline $\mathrm{H}(11)$ & $6401(16)$ & $-2675(14)$ & $1299(12)$ & $51(4)$ \\
\hline $\mathrm{H}(12)$ & $5241(15)$ & $-1245(13)$ & $782(12)$ & $45(4)$ \\
\hline $\mathrm{H}(131)$ & $1732(14)$ & $2988(12)$ & $167(11)$ & $40(4)$ \\
\hline $\mathrm{H}(132)$ & $2210(15)$ & $3879(13)$ & $1312(11)$ & $40(4)$ \\
\hline $\mathrm{H}(141)$ & $-366(15)$ & 2071(13) & 494(11) & $41(4)$ \\
\hline $\mathrm{H}(142)$ & $195(15)$ & $2878(13)$ & $1683(12)$ & $47(4)$ \\
\hline $\mathrm{H}(151)$ & $-74(15)$ & $4649(13)$ & $1206(11)$ & $46(4)$ \\
\hline $\mathrm{H}(152)$ & $-600(14)$ & $3902(12)$ & $64(11)$ & $37(4)$ \\
\hline $\mathrm{H}(161)$ & $-2650(20)$ & $2903(17)$ & $357(14)$ & $75(6)$ \\
\hline $\mathrm{H}(162)$ & $-2100(20)$ & $3701(18)$ & $1537(16)$ & $89(7)$ \\
\hline $\mathrm{H}(171)$ & $-3870(20)$ & $4589(15)$ & $684(13)$ & $70(5)$ \\
\hline $\mathrm{H}(172)$ & $-2990(20)$ & $4642(17)$ & $-119(15)$ & $80(6)$ \\
\hline $\mathrm{H}(173)$ & $-2360(20)$ & $5468(18)$ & $1040(14)$ & $77(6)$ \\
\hline $\mathrm{H}(211)$ & $3307(13)$ & $8113(11)$ & $4042(9)$ & $28(3)$ \\
\hline $\mathrm{H}(221)$ & $2330(15)$ & $9890(12)$ & $3870(10)$ & $39(4)$ \\
\hline $\mathrm{H}(222)$ & $1304(15)$ & $9280(12)$ & $4381(11)$ & $41(4)$ \\
\hline $\mathrm{H}(23)$ & $-437(15)$ & $8381(12)$ & $2902(10)$ & $38(4)$ \\
\hline $\mathrm{H}(241)$ & $164(16)$ & $7624(13)$ & $1437(11)$ & $47(4)$ \\
\hline $\mathrm{H}(242)$ & $1532(15)$ & $8655(12)$ & $1693(11)$ & $41(4)$ \\
\hline $\mathrm{H}(25)$ & $2652(14)$ & $7112(11)$ & $2181(10)$ & $35(4)$ \\
\hline $\mathrm{H}(26)$ & $379(14)$ & $6816(11)$ & $3180(9)$ & $32(3)$ \\
\hline $\mathrm{H}(28)$ & $3988(13)$ & $6253(11)$ & $3246(9)$ & $28(3)$ \\
\hline $\mathrm{H}(29)$ & 4911(14) & $4686(11)$ & $3682(9)$ & $30(3)$ \\
\hline $\mathrm{H}(31)$ & $1144(15)$ & $3507(13)$ & $4217(10)$ & $40(4)$ \\
\hline $\mathrm{H}(32)$ & $172(15)$ & $5098(12)$ & $3729(10)$ & $36(4)$ \\
\hline $\mathrm{H}(331)$ & $759(15)$ & $10492(12)$ & $2513(10)$ & $40(4)$ \\
\hline $\mathrm{H}(332)$ & $-858(15)$ & $9750(12)$ & $1983(10)$ & $39(4)$ \\
\hline $\mathrm{H}(341)$ & $230(14)$ & $11129(11)$ & $4065(10)$ & $33(3)$ \\
\hline $\mathrm{H}(342)$ & $-1232(15)$ & $10154(12)$ & $3696(10)$ & $40(4)$ \\
\hline $\mathrm{H}(351)$ & $-829(15)$ & $12157(12)$ & $2903(10)$ & $37(4)$ \\
\hline $\mathrm{H}(352)$ & $-2297(14)$ & $11220(12)$ & $2589(10)$ & $35(3)$ \\
\hline $\mathrm{H}(361)$ & $-1020(14)$ & $12854(11)$ & $4598(10)$ & $35(3)$ \\
\hline $\mathrm{H}(362)$ & $-2468(15)$ & $11831(12)$ & $4307(10)$ & $37(4)$ \\
\hline $\mathrm{H}(371)$ & $-3115(16)$ & $13750(13)$ & $4303(12)$ & $51(4)$ \\
\hline $\mathrm{H}(372)$ & $-3715(16)$ & $12814(13)$ & $3186(12)$ & $49(4)$ \\
\hline $\mathrm{H}(373)$ & $-2292(18)$ & $13840(15)$ & $3456(13)$ & $63(5)$ \\
\hline
\end{tabular}


Table 6. Torsion angles $\left[{ }^{\circ}\right]$ for exo,exo-Bicyclo[3.1.0]hexan-3,6-dicarbonsäure-3,6-di(4- $n$-pentylphenyl)ester $35 a$.

\begin{tabular}{|c|c|c|c|}
\hline $\mathrm{C}(5)-\mathrm{C}(1)-\mathrm{C}(2)-\mathrm{C}(3)$ & $19.39(12)$ & $\mathrm{C}(25)-\mathrm{C}(21)-\mathrm{C}(22)-\mathrm{C}(23)$ & $19.00(13)$ \\
\hline $\mathrm{C}(6)-\mathrm{C}(1)-\mathrm{C}(2)-\mathrm{C}(3)$ & $-45.54(13)$ & $\mathrm{C}(26)-\mathrm{C}(21)-\mathrm{C}(22)-\mathrm{C}(23)$ & $-46.42(14)$ \\
\hline $\mathrm{C}(1)-\mathrm{C}(2)-\mathrm{C}(3)-\mathrm{C}(13)$ & $-156.95(10)$ & $\mathrm{C}(21)-\mathrm{C}(22)-\mathrm{C}(23)-\mathrm{C}(33)$ & $-156.06(10)$ \\
\hline $\mathrm{C}(1)-\mathrm{C}(2)-\mathrm{C}(3)-\mathrm{C}(4)$ & $-30.71(12)$ & $\mathrm{C}(21)-\mathrm{C}(22)-\mathrm{C}(23)-\mathrm{C}(24)$ & $-29.58(13)$ \\
\hline $\mathrm{C}(13)-\mathrm{C}(3)-\mathrm{C}(4)-\mathrm{C}(5)$ & $156.33(10)$ & $\mathrm{C}(33)-\mathrm{C}(23)-\mathrm{C}(24)-\mathrm{C}(25)$ & $155.87(11)$ \\
\hline $\mathrm{C}(2)-\mathrm{C}(3)-\mathrm{C}(4)-\mathrm{C}(5)$ & $30.47(12)$ & $\mathrm{C}(22)-\mathrm{C}(23)-\mathrm{C}(24)-\mathrm{C}(25)$ & $29.30(13)$ \\
\hline $\mathrm{C}(2)-\mathrm{C}(1)-\mathrm{C}(5)-\mathrm{C}(4)$ & $-0.14(13)$ & $C(22)-C(21)-C(25)-C(24)$ & $-0.59(13)$ \\
\hline $\mathrm{C}(6)-\mathrm{C}(1)-\mathrm{C}(5)-\mathrm{C}(4)$ & $111.50(11)$ & $\mathrm{C}(26)-\mathrm{C}(21)-\mathrm{C}(25)-\mathrm{C}(24)$ & $111.25(11)$ \\
\hline $\mathrm{C}(2)-\mathrm{C}(1)-\mathrm{C}(5)-\mathrm{C}(6)$ & $-111.64(11)$ & $\mathrm{C}(22)-\mathrm{C}(21)-\mathrm{C}(25)-\mathrm{C}(26)$ & $-111.85(11)$ \\
\hline $\mathrm{C}(3)-\mathrm{C}(4)-\mathrm{C}(5)-\mathrm{C}(1)$ & $-19.12(13)$ & $\mathrm{C}(23)-\mathrm{C}(24)-\mathrm{C}(25)-\mathrm{C}(21)$ & $-17.98(14)$ \\
\hline $\mathrm{C}(3)-\mathrm{C}(4)-\mathrm{C}(5)-\mathrm{C}(6)$ & $46.27(14)$ & $\mathrm{C}(23)-\mathrm{C}(24)-\mathrm{C}(25)-\mathrm{C}(26)$ & $47.27(14)$ \\
\hline $\mathrm{C}(1)-\mathrm{C}(5)-\mathrm{C}(6)-\mathrm{C}(7)$ & $-110.07(12)$ & $\mathrm{C}(21)-\mathrm{C}(25)-\mathrm{C}(26)-\mathrm{C}(27)$ & $-106.19(12)$ \\
\hline $\mathrm{C}(4)-\mathrm{C}(5)-\mathrm{C}(6)-\mathrm{C}(7)$ & $153.72(11)$ & $\mathrm{C}(24)-\mathrm{C}(25)-\mathrm{C}(26)-\mathrm{C}(27)$ & $157.99(11)$ \\
\hline $\mathrm{C}(4)-\mathrm{C}(5)-\mathrm{C}(6)-\mathrm{C}(1)$ & $-96.22(12)$ & $\mathrm{C}(24)-\mathrm{C}(25)-\mathrm{C}(26)-\mathrm{C}(21)$ & $-95.82(12)$ \\
\hline $\mathrm{C}(5)-\mathrm{C}(1)-\mathrm{C}(6)-\mathrm{C}(7)$ & $109.45(12)$ & $\mathrm{C}(25)-\mathrm{C}(21)-\mathrm{C}(26)-\mathrm{C}(27)$ & $111.04(12)$ \\
\hline $\mathrm{C}(2)-\mathrm{C}(1)-\mathrm{C}(6)-\mathrm{C}(7)$ & $-155.12(11)$ & $\mathrm{C}(22)-\mathrm{C}(21)-\mathrm{C}(26)-\mathrm{C}(27)$ & $-152.64(11)$ \\
\hline $\mathrm{C}(2)-\mathrm{C}(1)-\mathrm{C}(6)-\mathrm{C}(5)$ & $95.42(11)$ & $\mathrm{C}(22)-\mathrm{C}(21)-\mathrm{C}(26)-\mathrm{C}(25)$ & $96.32(12)$ \\
\hline$C(5)-C(6)-C(7)-C(12)$ & $26.95(18)$ & $\mathrm{C}(25)-\mathrm{C}(26)-\mathrm{C}(27)-\mathrm{C}(32)$ & $-155.18(11)$ \\
\hline $\mathrm{C}(1)-\mathrm{C}(6)-\mathrm{C}(7)-\mathrm{C}(12)$ & $-43.26(17)$ & $\mathrm{C}(21)-\mathrm{C}(26)-\mathrm{C}(27)-\mathrm{C}(32)$ & $135.93(11)$ \\
\hline $\mathrm{C}(5)-\mathrm{C}(6)-\mathrm{C}(7)-\mathrm{C}(8)$ & $-152.60(11)$ & $\mathrm{C}(25)-\mathrm{C}(26)-\mathrm{C}(27)-\mathrm{C}(28)$ & $27.27(17)$ \\
\hline $\mathrm{C}(1)-\mathrm{C}(6)-\mathrm{C}(7)-\mathrm{C}(8)$ & $137.18(12)$ & $\mathrm{C}(21)-\mathrm{C}(26)-\mathrm{C}(27)-\mathrm{C}(28)$ & $-41.62(16)$ \\
\hline $\mathrm{C}(12)-\mathrm{C}(7)-\mathrm{C}(8)-\mathrm{C}(9)$ & $-1.87(17)$ & $\mathrm{C}(32)-\mathrm{C}(27)-\mathrm{C}(28)-\mathrm{C}(29)$ & $0.03(18)$ \\
\hline $\mathrm{C}(6)-\mathrm{C}(7)-\mathrm{C}(8)-\mathrm{C}(9)$ & $177.71(10)$ & $\mathrm{C}(26)-\mathrm{C}(27)-\mathrm{C}(28)-\mathrm{C}(29)$ & $177.60(11)$ \\
\hline $\mathrm{C}(7)-\mathrm{C}(8)-\mathrm{C}(9)-\mathrm{C}(10)$ & $-0.04(17)$ & $\mathrm{C}(27)-\mathrm{C}(28)-\mathrm{C}(29)-\mathrm{C}(30)$ & $-0.47(17)$ \\
\hline $\mathrm{C}(8)-\mathrm{C}(9)-\mathrm{C}(10)-\mathrm{C}(11)$ & $1.78(18)$ & $\mathrm{C}(28)-\mathrm{C}(29)-\mathrm{C}(30)-\mathrm{C}(31)$ & $0.10(17)$ \\
\hline $\mathrm{C}(8)-\mathrm{C}(9)-\mathrm{C}(10)-\mathrm{C}(18)$ & $-178.64(11)$ & $\mathrm{C}(28)-\mathrm{C}(29)-\mathrm{C}(30)-\mathrm{C}(38)$ & $179.12(11)$ \\
\hline $\mathrm{C}(9)-\mathrm{C}(10)-\mathrm{C}(11)-\mathrm{C}(12)$ & $-1.5(2)$ & $\mathrm{C}(29)-\mathrm{C}(30)-\mathrm{C}(31)-\mathrm{C}(32)$ & $0.71(18)$ \\
\hline $\mathrm{C}(18)-\mathrm{C}(10)-\mathrm{C}(11)-\mathrm{C}(12)$ & $178.88(12)$ & $\mathrm{C}(38)-\mathrm{C}(30)-\mathrm{C}(31)-\mathrm{C}(32)$ & $-178.31(11)$ \\
\hline $\mathrm{C}(10)-\mathrm{C}(11)-\mathrm{C}(12)-\mathrm{C}(7)$ & $-0.4(2)$ & $\mathrm{C}(30)-\mathrm{C}(31)-\mathrm{C}(32)-\mathrm{C}(27)$ & $-1.17(18)$ \\
\hline $\mathrm{C}(8)-\mathrm{C}(7)-\mathrm{C}(12)-\mathrm{C}(11)$ & $2.11(19)$ & $\mathrm{C}(28)-\mathrm{C}(27)-\mathrm{C}(32)-\mathrm{C}(31)$ & $0.79(18)$ \\
\hline $\mathrm{C}(6)-\mathrm{C}(7)-\mathrm{C}(12)-\mathrm{C}(11)$ & $-177.45(12)$ & $\mathrm{C}(26)-\mathrm{C}(27)-\mathrm{C}(32)-\mathrm{C}(31)$ & $-176.87(11)$ \\
\hline $\mathrm{C}(2)-\mathrm{C}(3)-\mathrm{C}(13)-\mathrm{C}(14)$ & $-177.84(11)$ & $\mathrm{C}(22)-\mathrm{C}(23)-\mathrm{C}(33)-\mathrm{C}(34)$ & $-58.84(15)$ \\
\hline $\mathrm{C}(4)-\mathrm{C}(3)-\mathrm{C}(13)-\mathrm{C}(14)$ & $62.06(14)$ & $\mathrm{C}(24)-\mathrm{C}(23)-\mathrm{C}(33)-\mathrm{C}(34)$ & $-179.69(11)$ \\
\hline $\mathrm{C}(3)-\mathrm{C}(13)-\mathrm{C}(14)-\mathrm{C}(15)$ & $173.26(10)$ & $\mathrm{C}(23)-\mathrm{C}(33)-\mathrm{C}(34)-\mathrm{C}(35)$ & $-165.23(10)$ \\
\hline $\mathrm{C}(13)-\mathrm{C}(14)-\mathrm{C}(15)-\mathrm{C}(16)$ & $-178.86(13)$ & $\mathrm{C}(33)-\mathrm{C}(34)-\mathrm{C}(35)-\mathrm{C}(36)$ & $-178.02(11)$ \\
\hline $\mathrm{C}(14)-\mathrm{C}(15)-\mathrm{C}(16)-\mathrm{C}(17)$ & $-179.32(16)$ & $\mathrm{C}(34)-\mathrm{C}(35)-\mathrm{C}(36)-\mathrm{C}(37)$ & $-177.09(11)$ \\
\hline $\mathrm{C}(9)-\mathrm{C}(10)-\mathrm{C}(18)-\mathrm{N}(1)$ & $-21(13)$ & $\mathrm{C}(31)-\mathrm{C}(30)-\mathrm{C}(38)-\mathrm{N}(2)$ & $63(5)$ \\
\hline $\mathrm{C}(11)-\mathrm{C}(10)-\mathrm{C}(18)-\mathrm{N}(1)$ & $159(13)$ & $\mathrm{C}(29)-\mathrm{C}(30)-\mathrm{C}(38)-\mathrm{N}(2)$ & $-116(5)$ \\
\hline
\end{tabular}




\section{G. Literaturverzeichnis}

[1] F. Reinitzer, Monatsh. Chem. 1888, 9, 421-441.

[2] O. Lehmann, Z. Physik. Chem., 1889, 4, 462.

[3] G. H. Heilmeier, L. A. Zanoni and L. A. Barton, Proc, IEEE., 1968, 56, 1162.

[4] H. Budig, R. Paschke, S. Diele S, I. Letko, G. Pelzl , Bowl-shaped liquid crystals - new derivatives of cyclotriveratrylene and calix[4]arene. Ber. Bunsen-Ges. Phys. Chem. 1993, 97, 1355-1357.

[5] G. Heppke, On Banana Liquid Crystals Chirality by Achiral Molecules, Workshop des SFB 335, TU Berlin, 1997.

[6] J. J. Wysocke, J. Adams W. Haas, Phys. Rev. Lett., 1968, 20, 1024.

[7] M. Schadt, W. Helfrich, Appl. Phys. Lett. 1971, 18, 127-128.

[8] H. Kelker, B. Scheuerle, Angew. Chem. 1969, 81, 903-904.

[9] G. H. Heilmeier, L. A. Zanoni, J. E. Goldmacher, Liquid Crystals and Ordered Fluids (Ed. J. F. Johnson, R. S. Porter), Plenum, New York, 1970.

[10] H. Kelker, B. Scheuerle, R. Hatz, W. Bartsch., Angew. Chem. 1970, 82, 984-985; Angew. Chem. Int. Ed., 1970, 9, 962-963.

[11] H. J. Deutscher, F. Kuschel, H. Schubert, D. Demus, Patent DD105701, 1974. D. Demus, H. J. Deutscher, F. Kuschel, H. Schubert, Patent DE2429093, 1975.

[12] G. W. Gray, K. J. Harrison, J. A. Nash, Electon Lett. 1973, 9, 130-131; G. W. Gray, K. J. Harrison, J. A. Nash, J. Chem. Soc. Chem. Commun., 1974, 431-432; G. W. Gray, J. Phys. Paris 1975, 36, C1 336-337; G. W. Gray, A. Mosley, J. Chem. Soc. Perkin 2, 1976, 97-102.

[13] Cf. E. Jakeman, E. P. Raynes, Phys. Lett. 1972, 39A, 69.

[14] R. Eidenschink, D. Erdmann, J. Krause, L. Pohl, Angew. Chem. 1977, 89, 103-103; Angew. Chem. Int. Ed. 1977, 16, 100-100; L. Pohl, R. Eidenschink, G. Krause, D. Erdmann, Phys. Lett. 1977, 60A, 421-423.

[15] S. Sugimori, T. Kojima, T. Tsuji, Patent JP Sho 62-39136 T. Kojima, T. Tsuji, S. Sugimori, Patent JP Sho 63-46738.

[16] Y. Goto, T. Ogawa, S. Sawada, S. Sugimori, Mol. Liq. Cryst. 1991, 209, 1.

[17] N. A. Clark, S. T. Lagerwall, Appl. Phys. Lett. 1980, 36, 899-901.

[18] K. Miyazawa, Novel Liquid Crylstalline Compounds With Cyclopropane Rings, Dissertation, Universität Göttingen, 2000. 
[19] H. Michaelsen, Auf dem Weg zu [n]Cyclotriangulanen: Neue spiroanellierte Cyclopropanderivate - Die Spiropentaneinheit als Flügelgruppe in flüssigkristallinen Verbindungen, Dissertation, Universität Göttingen 1994.

[20] R. Langer, Synthese flüssigkristalliner Substanzen mit zentraler Azabicyclo[3.1.0] hexaneinheit, Diplomarbeit, Universität Göttingen, 2000.

[21] A. de Meijere, S. I. Kozhushkov, A. I. Savchenko in Titanium and Zirconium in Organic Synthesis, Hrsg. I. Marek, Wiley-VCH, Weinheim 2002.

[22] O. G. Kulinkovich, A. de Meijere, Chem. Rev. 2000, 100, 2789-2834.

[23] LiqCryst 4.3, LCI-PUBLISHER 1995 - 2003, http://www.lci-publisher.com.

[24] Jean-Rierre Deprés, Andrew E. Green, Org. Synth. 1998, 195-200.

[25] E. J. Grubbs, D. J. Lee, A. G. Bellettine, J. Org. Chem. 1966, 31, 4069-4071.

[26] K. C. Murdock, R. B. Angier J. Org. Chem. 1962, 27, 2395-2398.

[27] L. H. Amundsen, R. H. Mayer, L. S. Pitts, L. A. Malentacchi, J. Am. Chem. Soc. 1951, $73,2118-2121$.

[28] N. E. Searle, Org. Synth. Coll. Vol IV, 1963, 424-426.

[29] A. J. Heuser, A. F. Noels, A. J. Anciaux, P. Teyssié, Synth. Commun. 1976, 600-602.

[30] Y. S. Hon, R. C. Chang, Heterocycles, 1991, 32, 1089-1099.

[31] M. J. de Vos, A. Krief, J. Am. Chem. Soc., 1982, 104, 4282-4283.

[32] B. Neises, W. Steglich, Angew. Chem. 1978, 90, 556-557; Angew. Chem. Int. Ed. 1978, $17,522-523$.

[33] N. E. Neubert, Mol. Cryst. Liq. Cryst. 1982, 89, 93-117.

[34] D. Vedejs, N. S. Bennett, L. M. Conn, S. T. Diver, M. Gingras, S. Lin, P. A. Oliver, M. J. Peterson, J. Org. Chem. 1993, 58, 7286-7288.

[35] X. Creary, J. Am. Chem. Soc. 1980, 102, 1611-1618.

[36] W. J. Seitz, M. M. Hossain, Tetrahedron Lett. 1994, 35, 7561-7764.

[37] C. P. Casey, S. W. Polichnowski, A. J. Shusterman, C. R. Jones, J. Am. Chem. Soc. 1979, 7282-7292.

[38] R. A. Moss, J. Org. Chem. 1962, 27, 2683.

[39] G. L. Closs, J. J. Coyle, J. Org. Chem. 1966, 31, 3837.

[40] R. A. Moss, J. R. Whittle, P. J. Friedenreich, J. Org. Chem. 1969, 34, 2220.

[41] T. Hudlicky, D. B. Reddy, S. V. Govindan, J. Org. Chem., 1983, 48, 3422-3428.

[42] M. P. Doyle, Chem. Rev. 1986, 86, 919-939. 
[43] M. P. Doyle, M. A. McKervey, T. Ye, Modern Catalysts for Organic Synthesis with Diazo Compounds, Wiley, New York, 1997.

[44] J. Koo, M. S. Fish, G. N. Walker, J. Blake, Org. Synth. Coll. Vol. IV, 1963, 327-330.

[45] M. P. Doyle et al., J. Am. Chem. Soc. 1995, 117, 5763-5775.

[46] G. A. Olah, M. Arvanaghi, D. Vankar, G. K. Surya Prakash, Synthesis 1980, 662-663.

[47] L. Horner, H. Oediger, H. Hoffmann, Liebigs Ann. 1959, 626, 26-34.

[48] E. C. Taylor, A. McKillop, Adv. Org. Chem. 1970, 7, 1.

[49] L. F. Tietze, Th. Eicher, Reaktionen und Synthesen, 2. Aufl, Thieme, Stuttgart 1991, 60, C-6.

[50] S. Winstein et al., Tetrahedron Suppl. 1966, 8, 621-645.

[51] M. R. Netherton, G. C. Fu, Angew. Chem. 2002, 114, 4066-4068; A. C. Frisch, N. Shaika, A. Zapf, M. Beller, Angew. Chem. 2002, 114, 4218-4221.

[52] K. Nicolaou, J. Li, G. Zenke, Helv. Chim. Acta 2000, 83, 1977-2006.

[53] Nenitzescu, Necsoiu, J. Am. Chem. Soc. 1950, 72, 3483.

[54] J. Thomas, D. Clough, J. Pharm. Pharmacol. 1963, 15, 167-177.

[55] J. J. Wolff, G. Frenkling, K. Harms, Chem. Ber. 1991, 55-561.

[56] I. Paterson, I. Fleming, Tetrahedron Lett. 1979, 993-994.

[57] A. Alberola, B. Calvo, A. G. Ortega, M. Vincetne, S. G. Granda, J. F. Van de Maelen, J. Chem. Soc., Perkin Trans. 1 1991, 203-209.

[58] C. C. Price, J. M. Judge, Org. Synth. Coll. Vol. V, 1973, 255-258.

[59] G. M. Rubottom, J. M. Gruber, R. Marrero, H. D. Juve jr., C. W. Kim, J. Org. Chem., 1983, 48, 4940-4944; Tetrahedron Lett. 1978, 993-994.

[60] A. Alberola, B. Calvo, A. G. Ortega, M. Vincente, S. G. Granda, J. F. van der Maelen, J. Chem. Soc. Perkin Trans I, 1991, 203-210.

[61] J. Tsuji, T. Kazuhiro, I. Minami, I. Shimizu, T. L. 1984, 4782-4786.

[62 ] H. Praetorius, A. Vogts, Patent DE2036026, 1972.

[63] J. Nelson, E. Day, J. F. Thorpe, J. Chem. Soc. 1920, 117, 1465-1474 .

[64] W. J. Middleton, J. Org. Chem. 1975, 40, 574-578.

[65] K. Burgess, W. A. van der Donk, M. B. Jarstfer, M. J. Ohlmeyer, J. Am. Chem. Soc. 1991, 113, 6139-6144; T. Jaime, F. Camps, J. Coll, E. Enric, N. Pascual, Tetrahedron 1992, 48, 9809-9818; D. F. Shellhamer, A. A. Briggs, B. M. Miller, J. M. Prince, D. H. Scott, V. L. Heasley, J. Chem. Soc. Perkin Trans.2 1996, 5, 973-978.

[66] D. H. R. Barton, S. W. McCombie, J. Chem. Soc. Perkin 1, 1975, 1574-1585. 
[67] T. W. Hanks, R. A. Ekeland, K. Emerson, R. D. Larsen, Organometal. 1987, 6, 28-32 ; F. F. Stewart, W. D. Neilson, R. E. Ekeland, R. D. Larsen, Organometal. 1993, 12, 45854589.

[68] D. N. Kursanov, G. I. Bolestova, U. G. Ibatullin, E. A. Kuramshina, Z. N. Parnes, J. Org. Chem. USSR (Engl. Trans.) 1985, 21, 2078-2085.

[69] G. Wagner, J. Russ. Phys. Chem. Soc., 1899, 31, 690; T. Kobayashi, Y. Uchiyama, J. Chem. Soc. Perkin 1, 2000, 2731; B. M. Trost, T. Yasukata, J. Am. Chem. Soc. 2001, 123,7162 .

[70] M. P. Doyle, Comprehensive Organometallic Chemistry II, Pergamon Press, New York, 1995, Vol. 12, Kap. 5.2.

[71] E. Christopher, N. Sheppart, D. B. Powell, J. R. Norton, W. Fischer et al., J. Am. chem. Soc. 1994, 7, 3058-3062.

[72] S. Arora, P. Binger, Synth. Commun. 1974, 801-803; A. de Meijere, S. I. Kozhushkov, T. Späth, N. S. Zafirov, J. Org. Chem. 1993, 58, 502-505.

[73] T. J. Hall, J. H. Hargis, J. Org. Chem. 1986, 51, 4185-4189.

[74] E. A. Werner, Chem. Ber. 1919, 115, 1093-1102.

[75] R. Moschel, W. Hudgins, A. Dipple, C. Robert, J. Org. Chem. 1986, 51, 4180-4185.

[76] X. Creary, J. Am. Chem. Soc. 1980, 45, 1611-1618.

[77] P. Helquist in Comprehensive Organic Synthesis, Vol. 4, (Hrsg: B. M. Trost, I. Fleming, M. F. Semmelhack), Pergamon Press, Oxford, 1991, pp. 951-995.

[78] M. P. Doyle, C. S. Seterson, D. L. Parker, Angew. Chem. Int. Ed. 1996, 35, 1334-1336.

[79] H. M. L. Davies, B. D. Doan, Tetrahedron Lett. 1996, 37, 3967; P. Bulugahapitiya, Y. Landais, L. Parra-Rapado, J. Org. Chem. 1997, 62, 1630; R. Buck, M. Doyle, M. Drysdale, Tetrahedron Lett. 1996, 37, 731; H. M. L. Davies, T. Hansen, J. Am. Chem. Soc. 1997, 119, 9075.

[80] T. Tsuji, S. Nishida, The Chemistry of the Cyclopropyl Group (Ed: Z. Pappoport), Wiley, New York, 1987, Part 1, 307; D. S. Wulfman, G. Linstrumelle, C. F. Cooper, The Chemistry of Diazonium and Diazo Groups (Ed. S. Patai), Wiley, Chichester, 1978, Part 2, p. 82 .

[81] S. M. McElvain, P. L. Weyna, J. Am. Chem. Soc. 1959, 83, 2579-2588.

[82] R. A. Moss, J. Org. Chem. 1962, 27, 2683-2685 .

[83] G. L. Closs, J. J. Coyle, J. Am. Chem. Soc. 1966, 31, 2759-2765. 
[84] J. E. Hodgkins, J. D. Woodyard, D. L. Stephenson, J. Am. Chem. Soc. 1964, 86, 40804085 .

[85] S. S. Hecht, A. C. Cope, J. Am. Chem. Soc. 1967, 89, 6920-6925.

[86] G. L. Closs, R. A. Moss, J. Am. Chem. Soc. 1964, 86, 4042-4053.

[87] G. L. Closs, S. H. Goh, J. Chem. Soc. Perkin Trans I, 1972, 2103-2108.

[88] G. P. Sharnin, V. V. Nurgatin, V. M. Ginzberg, Zh, Org. Khim. 1979, 15, 1635.

[89] H. Yanagawa, T. Kato, Y. Kitahara, Tetrahedron Lett. 1973, 14, 2137-2140.

[90] A. Pfaltz, Acc. Chem. Res. 1993, 26, 339-345; H. Fritschi, U. Leutenegger, A. Pfaltz, Helv. Chim. Acta 1988, 71, 1553-1565.

[91] M. P. Doyle, L. C. Wang, K. L. Loh, Tetrahedron Lett. 1984, 25, 4087-4090.

[92] K . Ito, T. Katsuki, Tetrahedron Lett. 1993, 34, 2661-2664; K .Ito, T. Katsuki, Synlett 1993, 638-640; K . Ito, M. Yoshitake, T. Katsuki, Chem. Lett. 1995, 1027-1028; K . Ito, T. Katsuki, Tetrahedron Lett. 1993, 34, 2661-2664; K. Ito, M. Yoshitake, T. Katsuki, Heterocycles 1996, 42, 305-317; K . Ito, M. Yoshitake, T. Katsuki, Tetrahedron 1996, $52,3905-3920$.

[93] M. G. Voronkov, E. N. Deryagina, G. M. Ivanova, Zh. Prikl. Khim. 1979, 55, 2625.

[94] W. Kirmse, M. Kapps, Chem. Ber. 1968, 101, 994-1003.

[95] A. J. Anciaux, A. J. Hubert, A. F. Noels, N. Petiniot, P. Teyssié, J. Org. Chem. 1980, 45, $695-702$.

[96] M. K. Gadziev, Prepr. Akad. Nauk. Gruz. SSR, 1991.

[97] G. Maas, Top. Curr. Chem. 1987, 137, 76-253.

[98] S. Dong, C. Tschierske, S. Diele, I. Wirth, Chem. Commun. 1998, 23, 2573-2574;

S. Dong, S. Diele, G. Pelzl, I. Wirth. C. Tschierske, J. Mat. Chem. 1999, 661-672.

[99] A. Rastegar, I. Musevic, M. Copic, T. Rasing, Proc. Soc. Opt. Eng. 1998, 3318, 118 121; A. Rastegar, T. Rasing, I. Musevic, G. Heppke, Phys. Rev. E 1999, 60(6-A), 6788-6792.

[100] M. E. Neubert, Mol. Cryst. Liq. Cryst. 1982, 89, 93-117.

[101] W. Maier, G. Meier, Z. Naturforsch. 1961, 16a, 262.

[102] K. Nicolaou, J. Li, G. Zenke, Helv. Chim. Acta 2000, 83, 1977-2006. 


\section{Danksagungen}

Für den wissenschaftlichen Unterricht an den Universitäten Saarbrücken und Göttingen danke ich den Herren Professoren und Privatdozenten U. Beifuß, R. Berger, E. Blasius, R. Brückner, Th. Eicher, W. Hack, L. Heck, G. F. Kahl, U. Klingebiel, H. Laatsch, K. Luther, J. Magull, A. de Meijere, A. Meller, H. W. Roesky, P. Schreiner, J. Schröder, M. Suhm, G. M. Sheldrick, L. F. Tietze, J. Troe, M. Veith, T. Wichert.

Herrn Dr. Remberg, Herrn Dr. H. Frauendorf und Frau G. Udvarnoki danke ich für die Aufnahmen der Massenspektren und ihr freundliches Interesse an diesbezüglichen Problemen. F. Hambloch danke ich für die Durchführung der Elementaranalysen. Herrn O. Senge danke ich für die Hilfe bei HPLC-Trennungen.

Herrn Prof. Dr. J. Magull und Herrn Dr. D. S. Yufit danke ich für die Durchführung der Röntgen-Strukturanalysen.

Ich bedanke mich bei meinen Laborkollegen Dr. Christian Funke, Dr. Markus Kordes, M. Maywald, Dr. A. Savchenko, H. W. Sünnemann, T. Voigt und Dr. Craig Williams für das nette Miteinander im Labor 302.

Für viele Diskussionen über chemische Probleme und das Korrekturlesen dieser Arbeit danke ich Dr. S. I. Kozhushkov, Dr. A. Leonov, M. Schelper und G. D. Tebben.

Der Firma ICN Biomedicals GmbH danke ich für meine vierjährige Anstellung, die Einräumung freier Arbeitszeiten sowie die Ermöglichung vieler Fortbildungen.

S. Beußhausen, C. Rauch und G. Keil-Knepel sei für ihre stete Hilfsbereitschaft herzlich gedankt.

Ich bedanke mich besonders bei meinen Eltern für ihr Verständnis und ihre Unterstützung, ohne die dieses Studium nicht möglich gewesen wäre und bei Nadine Lippert für viele schöne Stunden in den vergangenen drei Jahren. 


\section{Lebenslauf}

Am 23. Januar 1971 wude ich als zweites Kind des Schreiners Ludwig Langer und seiner Ehefrau Elfriede Langer, geb. Funschler in Püttlingen/Saar geboren.

Im Herbst 1977 erfolgte die Einschulung in die Grundschule Altenkessel. Ich besuchte von 1981 bis 1983 das staatliche Marie-Luise-Kaschnitz-Gymnasium und bestand das Abitur 1991 am staatlichen humanistischen Ludwigsgymnasium in Saarbrücken.

Ich absolvierte meinen Zivildienst von 1991 bis 1992 bei der Arbeiterwohlfahrt in Saarbrücken.

Im Wintersemester 1992 nahm ich in Saarbrücken das Studium der Chemie auf, setzte es 1994 in Göttingen fort, wo ich von September 1999 bis Mai 2000 unter der wissenschaftlichen Leitung von Herrn Prof. Dr. A. de Meijere mit dem Thema „Synthese flüssigkristalliner Substanzen mit zentraler Azabicyclo[3.1.0]hexaneinheit" meine Diplomarbeit anfertigte, und den akademischen Grad „Dipl.-Chem“ erhielt.

Ich begann im Mai 2000 im Arbeitskreis von A. de Meijere in Kooperation mit Chisso Petrochemicals Co. die Arbeit an der Entwicklung der Bicyclo[3.1.0]hexane, deren Erfolge unter der japanischen Patentnummer 2002-59289 veröffentlicht wurden.

In der Zeit von Oktober 2000 bis Januar 2003 war ich als wissenschafliche Hilfkraft an der Universität Göttingen beschäftigt, wo ich Studenten der Chemie und der Biologie betreute, zuletzt als leitender Assistent.

Seit Mai 1999 bin ich bei der Firma ICN Biomedicals GmbH für die Erstellung von Produktinformationen entsprechend dem Chemikaliengesetz, dem Arzneimittelgesetz, dem Grundstoffüberwachungsgesetz, und der Transportinformationen gemäß ADR/RID in einer Teilzeitbeschäftigung angestellt.

Von April bis Juni 2002 nahm ich mit Erfolg an dem Kurs zur allgemeinen Pharmakologie und Toxikologie der Medizinischen Fakultät der Universität Göttingen teil. 
$\underline{\text { Substanzklassen }}$

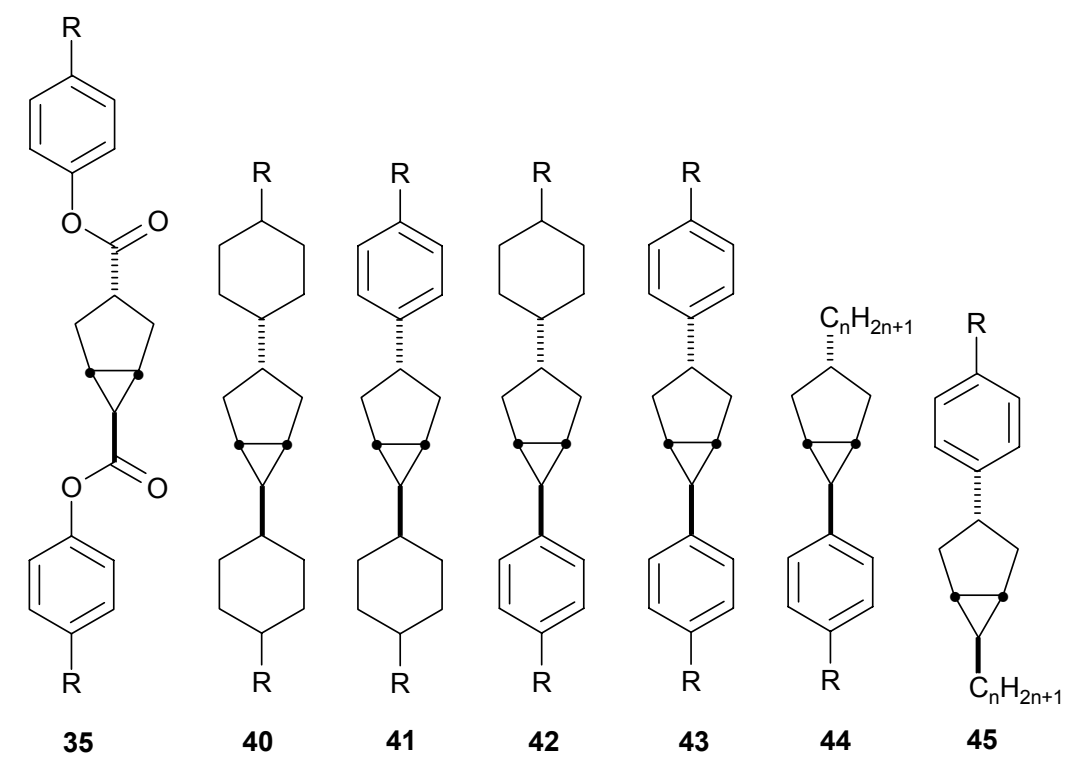

wichtige Intermediate

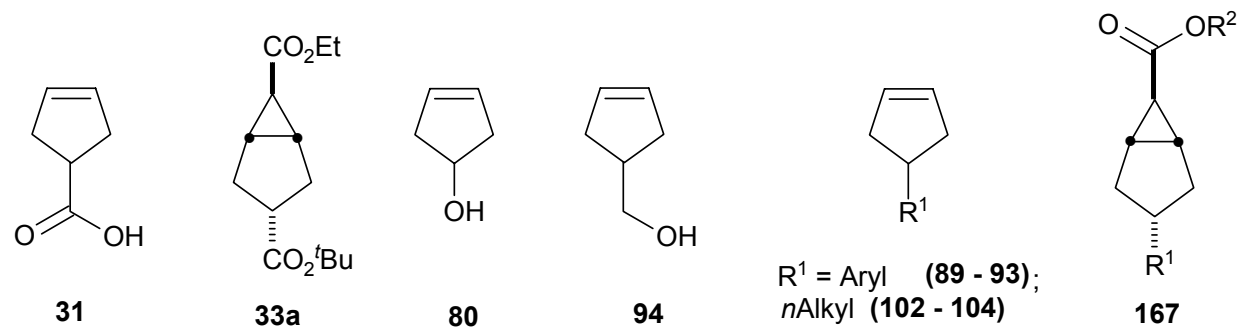

\section{Zielprodukte}

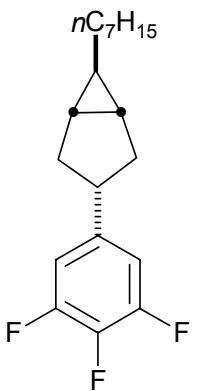

170

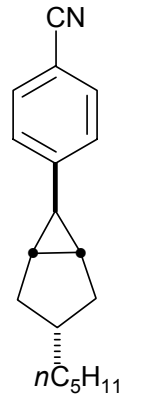

171<smiles>CCCCC1C2C[C@H]1C1C[C@H](CCc3ccc(OCC)c(F)c3F)CC21</smiles>

172

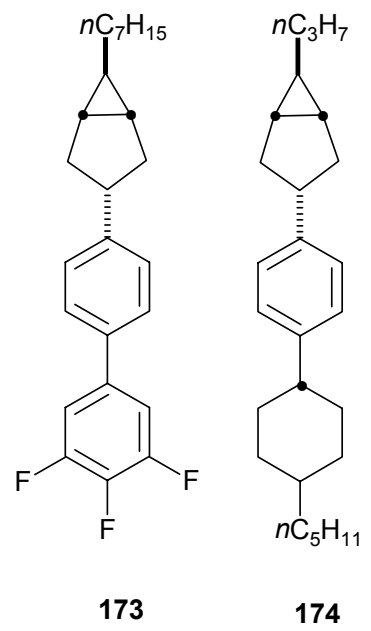

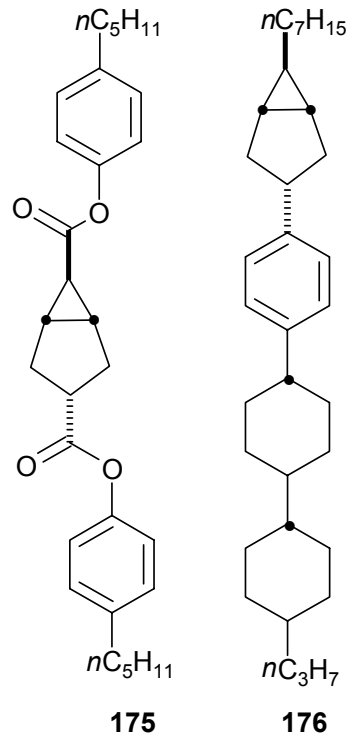

